\title{
Metal-Free Photoredox-Catalyzed C-H/C-H Coupling of Arenes Enabled by Interrupted Pummerer Activation
}

\author{
Miles Aukland, Mindaugas Šiaučiulis, Adam West, Gregory Perry, David Procter
}

Submitted date: 29/07/2019 - Posted date: 30/07/2019

Licence: CC BY-NC-ND 4.0

Citation information: Aukland, Miles; Šiaučiulis, Mindaugas; West, Adam; Perry, Gregory; Procter, David (2019): Metal-Free Photoredox-Catalyzed C-H/C-H Coupling of Arenes Enabled by Interrupted Pummerer Activation. ChemRxiv. Preprint.

Aryl-aryl cross-coupling constitutes one of the most widely used procedures for the synthesis of high-value materials, ranging from pharmaceuticals to organic electronics and conducting polymers. The assembly of (hetero)biaryl scaffolds generally requires multiple steps; coupling partners must be functionalized before the key bond-forming event is considered. Thus, the development of selective $\mathrm{C}-\mathrm{H}$ arylation processes in arenes, that side-step the need for prefunctionalized partners, is crucial for streamlining the construction of these key architectures. Here we report an expedient, one-pot assembly of (hetero)biaryl motifs using photocatalysis and two non-prefunctionalized arene partners. The approach is underpinned by the activation of a $\mathrm{C}-\mathrm{H}$ bond in an arene coupling partner using the interrupted Pummerer reaction. A unique pairing of the organic photoredox catalyst and the intermediate dibenzothiophenium salts enables highly selective reduction in the presence of sensitive functionalities. The utility of the metal-free, one-pot strategy is exemplified by the synthesis of a bioactive natural product and the modification of complex molecules of societal importance.

File list (2)

DJP-MA-manuscript-July8.pdf (11.06 MiB)

view on ChemRxiv - download file 


\title{
Metal-Free Photoredox-Catalyzed C-H/C-H Coupling of Arenes Enabled by Interrupted Pummerer Activation
}

\author{
Miles H. Aukland, ${ }^{1}$ Mindaugas Šiaučiulis, ${ }^{1}$ Adam West, ${ }^{1}$ Gregory J. P. Perry, ${ }^{1}$ and David J. Procter ${ }^{* 1}$
}

\begin{abstract}
Aryl-aryl cross-coupling constitutes one of the most widely used procedures for the synthesis of high-value materials, ranging from pharmaceuticals to organic electronics and conducting polymers. The assembly of (hetero)biaryl scaffolds generally requires multiple steps; coupling partners must be functionalized before the key bond-forming event is considered. Thus, the development of selective $\mathrm{C}-\mathrm{H}$ arylation processes in arenes, that side-step the need for prefunctionalized partners, is crucial for streamlining the construction of these key architectures. Here we report an expedient, one-pot assembly of (hetero)biaryl motifs using photocatalysis and two non-prefunctionalized arene partners. The approach is underpinned by the activation of a $\mathrm{C}-\mathrm{H}$ bond in an arene coupling partner using the interrupted Pummerer reaction. A unique pairing of the organic photoredox catalyst and the intermediate dibenzothiophenium salts enables highly selective reduction in the presence of sensitive functionalities. The utility of the metal-free, one-pot strategy is exemplified by the synthesis of a bioactive natural product and the modification of complex molecules of societal importance.
\end{abstract}

\section{Introduction}

The construction of (hetero)biaryls is a key application of cross-couplings, ${ }^{1}$ as the motif is ubiquitous amongst medicines, functional materials, and agrochemicals. ${ }^{2,3}$ Although powerful, traditional cross-couplings require two prefunctionalized partners and supply-risk platinum group metals. In order to streamline synthesis, it is desirable to engage non-prefunctionalized starting materials in cross-coupling processes that selectively manipulate $\mathrm{C}-\mathrm{H}$ bonds in order to access novel building blocks or modify complex structures at a late-stage. Methods for accessing (hetero)biaryl motifs through selective $\mathrm{C}-\mathrm{H} / \mathrm{C}-\mathrm{H}$ couplings have grown in sophistication in recent years, ${ }^{4}$

\footnotetext{
${ }^{1}$ School of Chemistry, University of Manchester, Oxford Road, Manchester, M13 9PL, UK.

* Corresponding author. david.j.procter@manchester.ac.uk.
} 
however, methods can still require platinum group metals, high temperatures and/or suffer from the need for prefunctionalization in the form of a directing group to ensure site selective $\mathrm{C}-\mathrm{H}$ functionalization. ${ }^{5}$ The use of photoredox catalysis has emerged as a complementary approach to (hetero)biaryls that operates under mild conditions and, in some cases, without the requirement for transition metals. While initial reports used aryl diazonium salts as substrates, ${ }^{6,7}$ recent progress has allowed less hazardous and more available substrates such as aryl halides to be used (Fig. 1a). ${ }^{8-13}$ A process that allowed the coupling of two non-prefunctionalized partners using photoredox catalysis would represent a clear advance. Here we present a general and effective photoredoxcatalyzed approach to achieve the chemo- and regioselective $\mathrm{C}-\mathrm{H} / \mathrm{C}-\mathrm{H}$ coupling of non-prefunctionalized arenes. The one-pot approach is underpinned by the selective interrupted Pummerer activation of an arene partner, using a benzothiophene $S$-oxide mediator, prior to catalytic cross-coupling with a second, non-prefunctionalized partner, mediated by an organic photocatalyst and visible light (Fig. 1b).

a

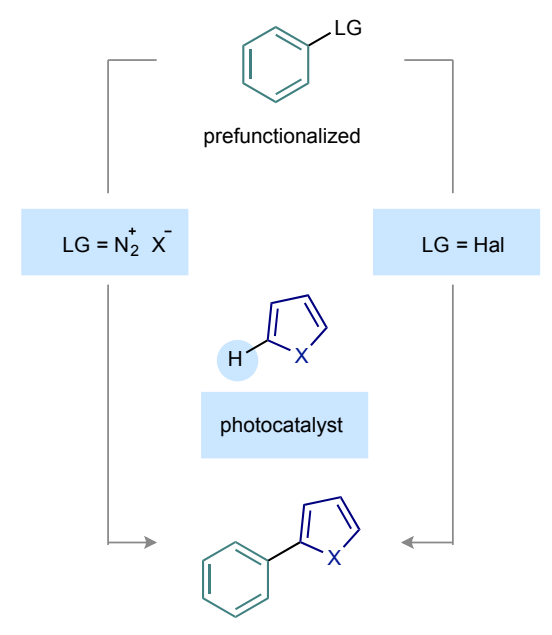

b

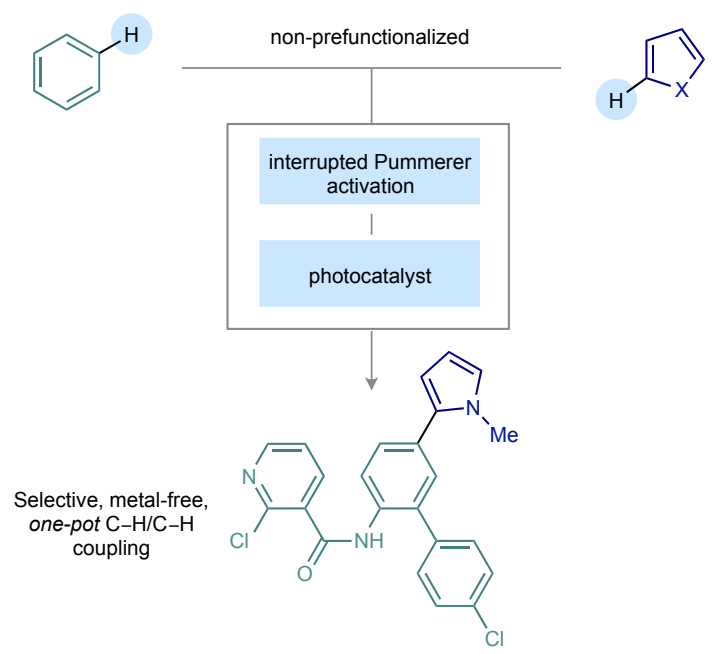

Figure 1. | Strategies for arylation of arenes. a, Photocatalytic approaches for the preparation of (hetero)biaryls. Such methods require prefunctionalized arene coupling partners, for example, aryl diazonium salts or aryl halides. b, Outline of a metal-free, strategy for the one-pot, photocatalytic $\mathrm{C}-\mathrm{H} / \mathrm{C}-\mathrm{H}$ coupling of non-prefunctionalized arenes that relies on a selective, interrupted Pummerer reaction to activate one partner. LG = Leaving group.

\section{Results and Discussion}


Owing to their reactivity at both oxygen and at sulfur, and the tuneability of such reactivity, sulfoxides are attracting significant attention as selective activators of partners for cross-coupling, often at the expense of $\mathrm{C}-\mathrm{H}$ bonds. ${ }^{14-36}$ We recently disclosed a strategy to activate the $\mathrm{C}-\mathrm{H}$ bonds of nucleophilic alkenes using sulfoxides. ${ }^{37}$ Sulfenylation by sulfoxide results in in situ polarity reversal of nucleophilic alkenes and the resultant alkenyl sulfonium salts are willing partners in nickel-catalyzed cross-couplings with organozincs that are carried out in the same pot. Herein, we report an effective, one-pot method for the selective $\mathrm{C}-\mathrm{H} / \mathrm{C}-\mathrm{H}$ cross-coupling of nonprefunctionalized arene partners through the marriage of interrupted Pummerer activation and organophotocatalysis (Fig. 2a). Exploiting commercial dibenzothiophene S-oxide (DBTSO) as the process mediator ensures high selectivity and high reactivity in both sulfenylation and aryl radical generation, and the by-product dibenzothiophene can be easily recovered and recycled. Key to the approach is the identification of 10phenylphenothiazine (PTH) as the optimal photocatalyst (see supporting information for full optimization studies and comparison with more conventional photoredox catalysts). The protocol involves direct reduction of the substrate by the excited catalyst and does not require super-stoichiometric quantities of electron donors (e.g. amines) to access the reactive state of the photocatalyst and enable efficient turnover. ${ }^{38-40}$

Our blueprint for the process begins with sulfoxide activation of the nucleophilic arene, by an interrupted Pummerer reaction, to generate the corresponding aryldibenzothiophenium salt ( $\mathrm{Ar}-\mathrm{DBT}^{+}$; Fig. $\left.2 \mathrm{~b}, \mathbf{A} \rightarrow \mathbf{B}\right)$. Single electron transfer (SET) from the excited state PTH to the Ar-DBT ${ }^{+}$results in the expulsion of dibenzothiophene and concomitant formation of an aryl radical species (Fig. $2 b, \mathbf{B} \rightarrow$ C). Sulfonium salts, ${ }^{41,42}$ in particular triarylsulfonium salts, ${ }^{43,33}$ have previously been investigated as aryl radical precursors using traditional ruthenium and iridium photocatalysts, but due to their inherent structure, they display non-selective $\mathrm{C}-\mathrm{S}$ bond cleavage and radical generation. Crucially, the choice of sulfonium salts formed from dibenzothiophene S-oxide (e.g. 2) is key as; i) SET reduction of the C-S bond is promoted by a gain in aromaticity, and; ii) SET reduction leads to selective cleavage of one of the $C-S$ bonds. After subsequent trapping of the aryl radical (Fig. $2 b, C \rightarrow D$ ), turnover of the catalytic cycle is thought to occur through oxidation of the newly formed radical adduct and aromatization delivers the product (Fig. 2 b, $\mathbf{D} \rightarrow \mathbf{E} \rightarrow \mathbf{F}$ ). At present, a radical chain process in which $\mathbf{D}$ reduces $\mathbf{B}$, to generate $\mathbf{C}$ and E, can't be ruled out. ${ }^{40}$ After optimisation (see supplementary information for full details), the metal-free, 
one-pot process was exemplified by the $\mathrm{C}-\mathrm{H} / \mathrm{C}-\mathrm{H}$-type coupling of tert-butylbenzene $\mathbf{1}$ and $\mathrm{N}$-methyl pyrrole to give (hetero)biaryl 3 (Fig. 2c).

a
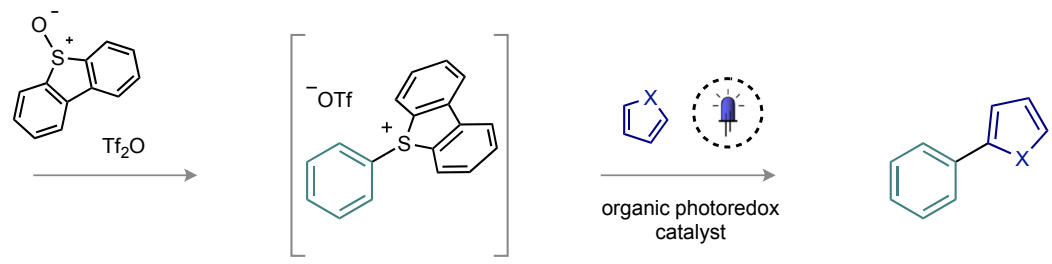

b
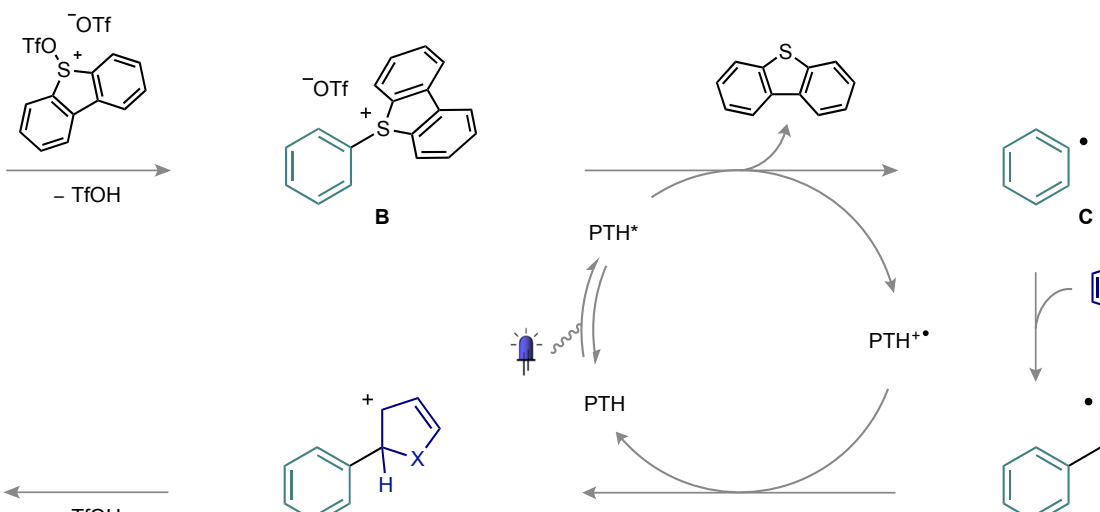<smiles>c1ccccc1</smiles>

B<smiles>Fc1cccc(-c2ccccc2)c1</smiles><smiles>[X]C1(c2ccccc2)[CH+]C=CC1</smiles>

$\mathrm{E}$

c
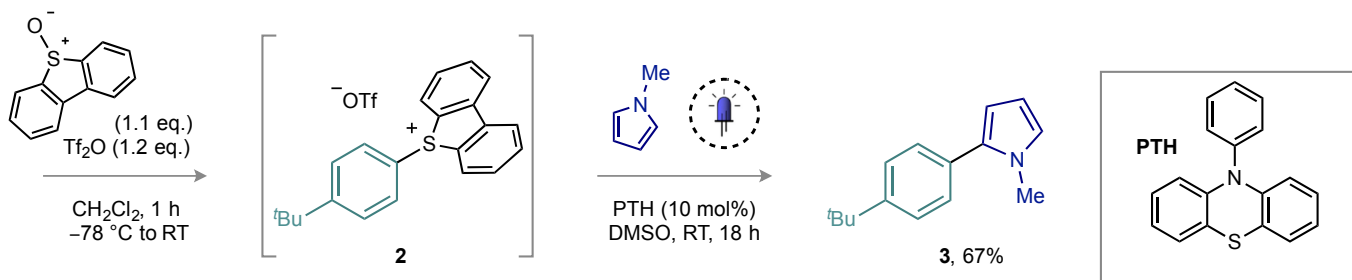

Figure 2. | Mechanistic proposal for a one-pot, metal-free, photoredox-catalyzed C-H/C-H coupling of arenes, enabled by interrupted Pummerer activation. a, Overview of the developed sequence. b, Mechanistic proposal: interrupted Pummerer activation of arene A generates the corresponding $\mathrm{Ar}-\mathrm{DBT}^{+}$salt $\mathbf{B}$. Single electron reduction of $\mathbf{B}$ releases aryl radical $\mathbf{C}$, which is coupled with the arene trap to form $\mathbf{D}$. Single electron oxidation of D may close the catalytic cycle and delivers the product of aryl-aryl coupling F. c, Exemplification of a one-pot, metal-free, photoredox-catalyzed $\mathrm{C}-\mathrm{H} / \mathrm{C}-\mathrm{H}$ coupling of arenes, enabled by interrupted Pummerer-activation, using tert-butylbenzene and $\mathrm{N}$-methylpyrrole. PTH, 10-phenylphenothiazine. 
Mechanistic investigations. To gain support for the proposed reaction mechanism, the feasibility of the SET event was assessed using cyclic voltammetry (CV). CV analysis of a range of dibenzothiophenium salts, showed irreversible reduction profiles with $\mathrm{E}^{\text {red }}=-1.1 \mathrm{~V}$ (versus saturated calomel electrode, $\mathrm{SCE}$ ) in DMSO (Supplementary Fig. 1, 2, and 3), significantly less negative than that determined for the excited state of PTH $\left(\mathrm{E}_{1 / 2}{ }^{*}=-2.1 \mathrm{~V}\right.$ versus SCE; Supplementary Fig. 5$)$ in DMSO. In line with these findings, Stern-Volmer experiments confirmed that $\mathrm{Ar}-\mathrm{DBT}^{+}$salt 2 effectively quenches the excited state of the photocatalyst (Fig. 3a and Supplementary Fig. 9 and 11). Radical scavenger and radical probe experiments were also used to probe the reaction mechanism. The presence of 2,2,6,6-tetramethylpiperidin-1-yl)oxyl (TEMPO) in the reaction mixture inhibited the formation of (hetero)biaryl 3 (Fig. 3b) while the addition of radical scavenger 1,1-diphenylethylene $(\mathrm{DPE})^{11,44}$ gave the product of radical trapping 4 (Fig. 3c). Finally, reduction of engineered $\mathrm{Ar}^{-\mathrm{DBT}^{+}}$salt $\mathbf{5}$, containing an ortho-allyl ether moiety, gave additional support for the proposed mechanism; 5-exo-trig radical cyclization/addition product 6 was obtained in moderate yield (Fig. 3d).

a

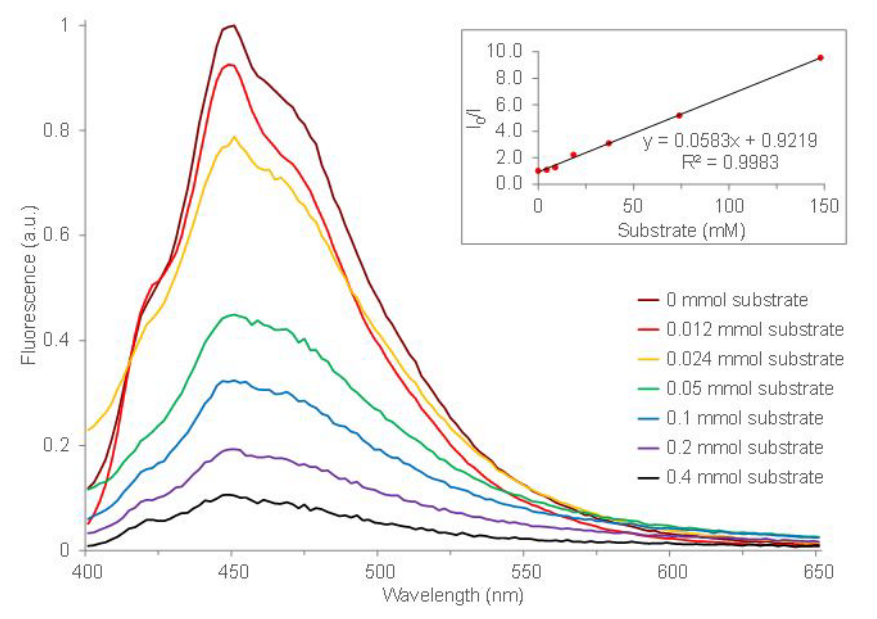

b

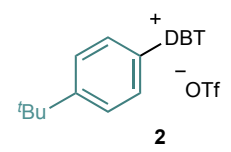

C

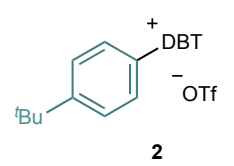

d

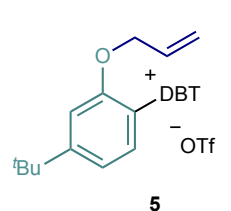

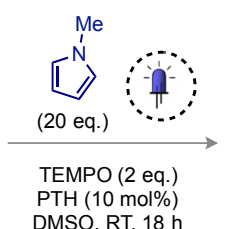
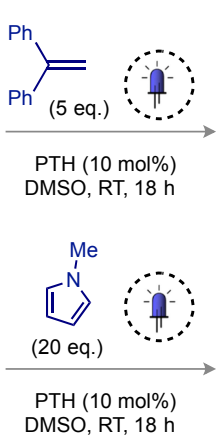

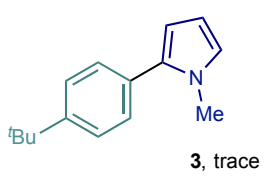

3, trace

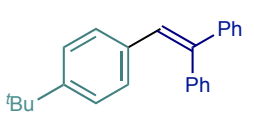

4, $70 \%$

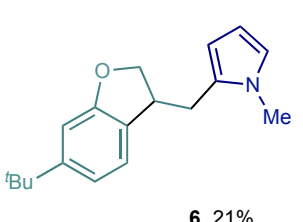

Figure 3. | Mechanistic investigations. a, Fluorescence studies show the quenching of PTH upon addition of Ar$\mathrm{DBT}^{+}$salt 2. b, The presence of TEMPO inhibits the photoredox $\mathrm{C}-\mathrm{H} / \mathrm{C}-\mathrm{H}$ coupling. $\mathrm{c}$, the postulated aryl radical intermediate can be intercepted by the radical trap 1,1-diphenylethene to afford alkenylated product $\mathbf{4}$. $\mathbf{d}$, An intramolecular radical trap experiment using the allyl ether-containing substrate $\mathbf{5}$ delivers the product or radical cyclization/addition 6. $\mathrm{DBT}^{+}$, dibenzothiophenium; TEMPO, 2,2,6,6-tetramethylpiperidin-1-yl)oxyl. 
Evaluation of the protocol's substrate scope. Having established optimal reaction conditions and gained support support for our mechanistic proposal, we set about determining the generality of the one-pot $\mathrm{C}-\mathrm{H} / \mathrm{C}-\mathrm{H}$ coupling (Table 1). Simple, alkyl-substituted arenes were arylated with $N$-methylpyrrole in good overall yields (3 and 7-10). More electron-rich phenol and aniline derivatives also underwent coupling in good yields over the two stages of the process (11-17). To assess the compatibility of various functional groups, a range of substituted anisoles were then engaged in the arylation sequence. Substrates bearing halogens, triflyl, mesyl, keto, amido, ester, trifluromethyl, and cyano groups all provided the desired heterobiaryls with complete chemoselectivity (18-28). The high chemoselectivity observed in the reduction of the $\mathrm{Ar}-\mathrm{DBT}^{+}$group is particularly noteworthy, given that PTH has previously been shown to catalyse the reduction of aryl halides ${ }^{45,46}$ and trifluoromethyl groups. ${ }^{47}$ The process' tolerance of halide and triflyl functional groups signals its orthogonality with regard to traditional crosscoupling and ensures that further structural modification of products can be carried out using conventional crosscoupling processes. Furthermore, performing the preparation of $\mathbf{2 6}$ on a $10 \mathrm{mmol}$ scale, without any alterations to the reaction conditions or set-up, revealed that the protocol is scalable. 


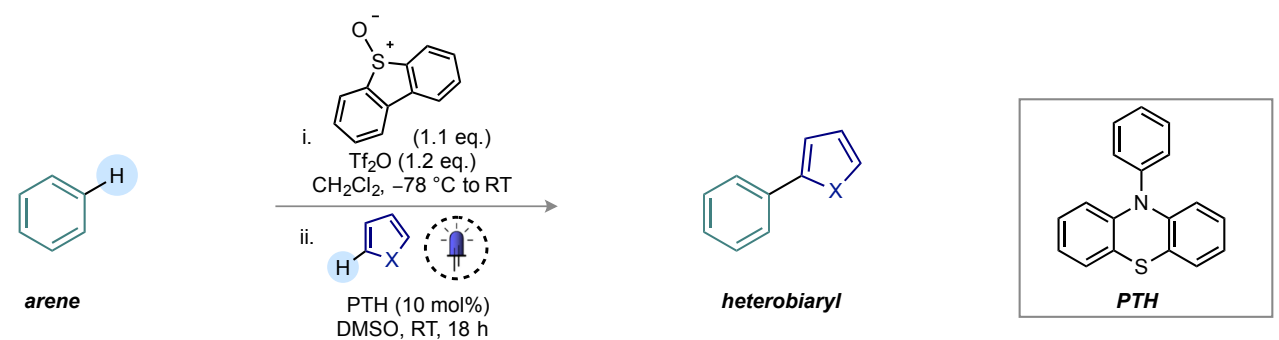

a Arenes

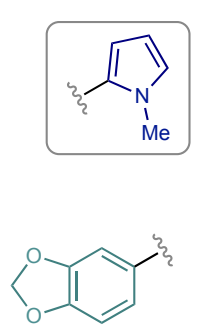

$11,62 \%$

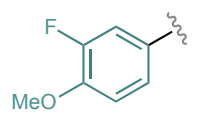

$18,67 \%$

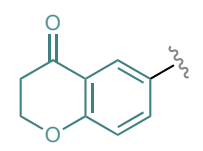

24, $48 \%$

b

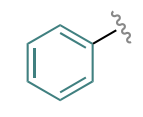

7, $49 \%^{a}$

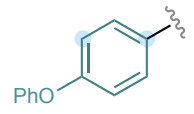

$12,67 \%$
$11: 1 \mathrm{rr}$

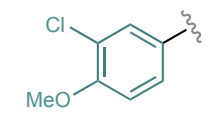

$19,68 \%$

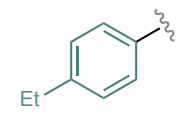

$8,52 \%$

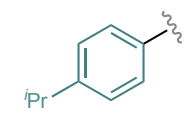

9, $49 \%$

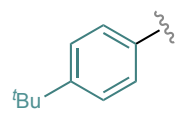

3, $51 \%$

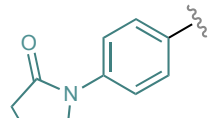

$14,60 \%$

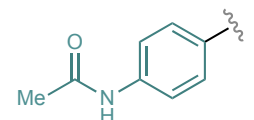

$15,54 \%$

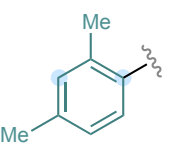

$10,51 \%$
$15: 1 \mathrm{rr}$

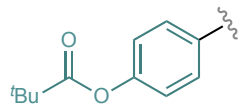

13, $51 \%$

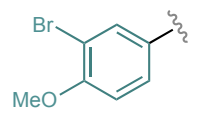

20, $69 \%$

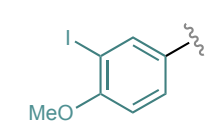

21, $64 \%$

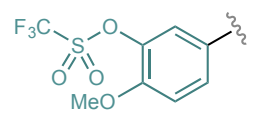

22, $86 \%$

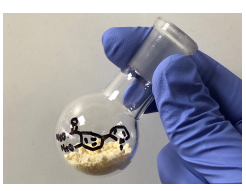

26, $59 \%(68 \%)^{b}$

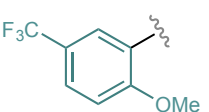

27, $82 \%$

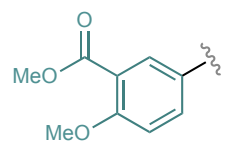

26, $67 \%$<smiles>CCNC(=O)c1cc(I)ccc1OC</smiles>

25, $64 \%$

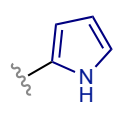

29, $52 \%$

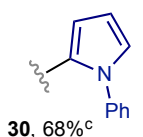

$30,68 \%{ }^{\mathrm{c}} \mathrm{Ph}$

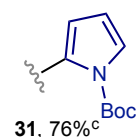

$31,76 \% \mathrm{c}^{\mathrm{Boc}}$

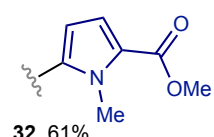

32, $61 \%^{\mathrm{Me}}$

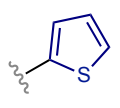

33, $59 \%$

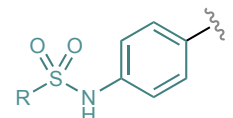

16, $R=p$-Tol, $58 \%$ 17, $\mathrm{R}=\mathrm{Me}, 56 \%$

23, $76 \%$

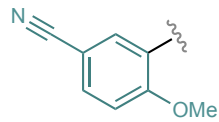

28, $71 \%$<smiles>CC(=O)c1csc(I)c1</smiles>

35, $65 \%$

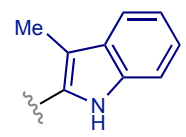

42, $52 \%$

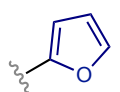

36, $57 \%$<smiles>Cc1cnccn1</smiles>

43, $42 \%$

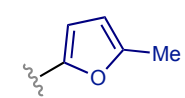

37, $53 \%$<smiles>COc1ccc([12CH3])c(OC)n1</smiles>

44, $42 \%$

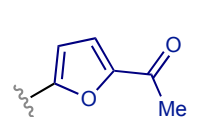

38, $70 \%$

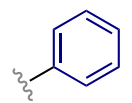

45, $55 \%^{a}$

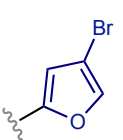

39, $63 \%$<smiles>Fc1ccc(F)c(I)c1</smiles>

46, $56 \%^{a}$

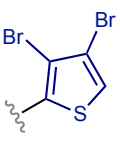

$34,62 \%$

Table 1. | Evaluation of the substrate scope in the metal-free, photoredox-catalyzed $\mathrm{C}-\mathrm{H} / \mathrm{C}-\mathrm{H}$ coupling of arenes, enabled by interrupted Pummerer activation. a, Substrate scope with respect to the arene coupling partner. b, Substrate scope with respect to the (hetero)arene radical trap. All reactions were run on $0.2 \mathrm{mmol}$ 
scale with 20 equivalents of the trapping reagent, unless stated otherwise. ${ }^{\mathrm{a}} 1 \mathrm{~mL}$ of radical trap used. ${ }^{\mathrm{b}}$ based on recovered starting material. ${ }^{c} 10$ equivalents of radical trap used.

We next evaluated the scope with respect to the trapping (hetero)arene. A range of substituted pyrroles (29-32), thiophenes (33-35), furans (36-40), benzofuran (41), and indole (42) partners was used successfully in the coupling. In the cases of 3-substituted heterocycles, complete regiocontrol was observed (35, 39, and 40). Ar$\mathrm{DBT}^{+}$salts can also engage in Minisci-type couplings (43). Finally, in addition to being capable of activation using the interrupted Pummerer process, arenes can also serve as radical trapping agents, thus allowing access to biaryls (45-48).

Application of the developed sequence. To showcase the potential of the one-pot, metal-free strategy, we have applied it to the synthesis and modification of several complex molecules. We first targeted the pseudilin marine natural products (Fig. 4a). Owing to their unusual, highly halogenated structures, pseudilins have gained attention in recent years and have been found to exhibit antibiotic, antitumor, and phytotoxic activities. ${ }^{48-50}$ Previous routes to the pseudilins have invariably used stepwise heterocycle construction from precursor benzaldehydes or phenyl ketones, with the shortest synthesis to date being seven steps in length. ${ }^{50}$ Engaging commercial 2,4dichloroanisole in our one-pot sequence delivered $\mathrm{N}-\mathrm{H}$ heterobiaryl 49 after removal of the tert-butoxycarbonyl group. Subsequent chlorination and demethylation provided natural, pentachloropseudilin $\mathbf{5 0}$, while bromination of the pyrrole ring enabled access to the dichloro-tribromopseudilin analogue 51, both in good overall yield.

Our method is also suited to the selective modification of complex scaffolds of biological significance (Fig. 4b). Salicin pentaacetate (52), boscalid (53), fenofibrate (54), clofibrate (55), and $N$-acetylmexiletine (56) underwent smooth arylation using the two-stage, one-pot process. Complete chemo- and regiocontrol was observed in all cases. The structure of $\mathbf{5 3}$ was confirmed by X-ray crystallographic analysis. 
a
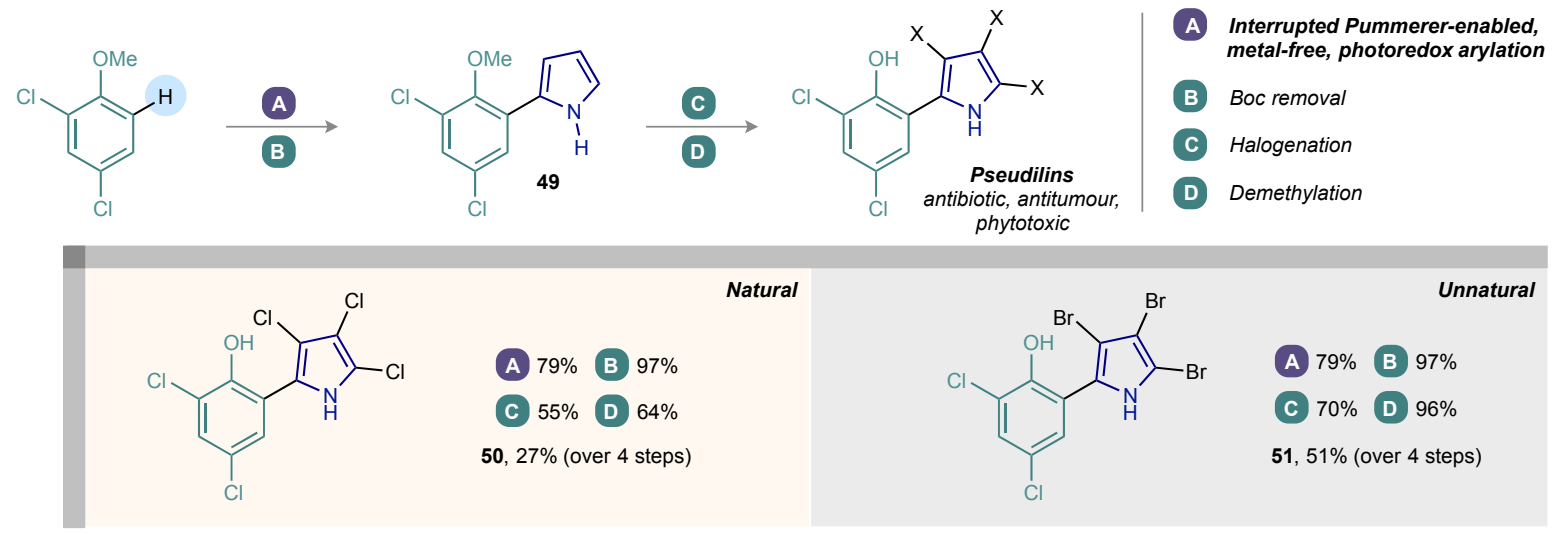

b
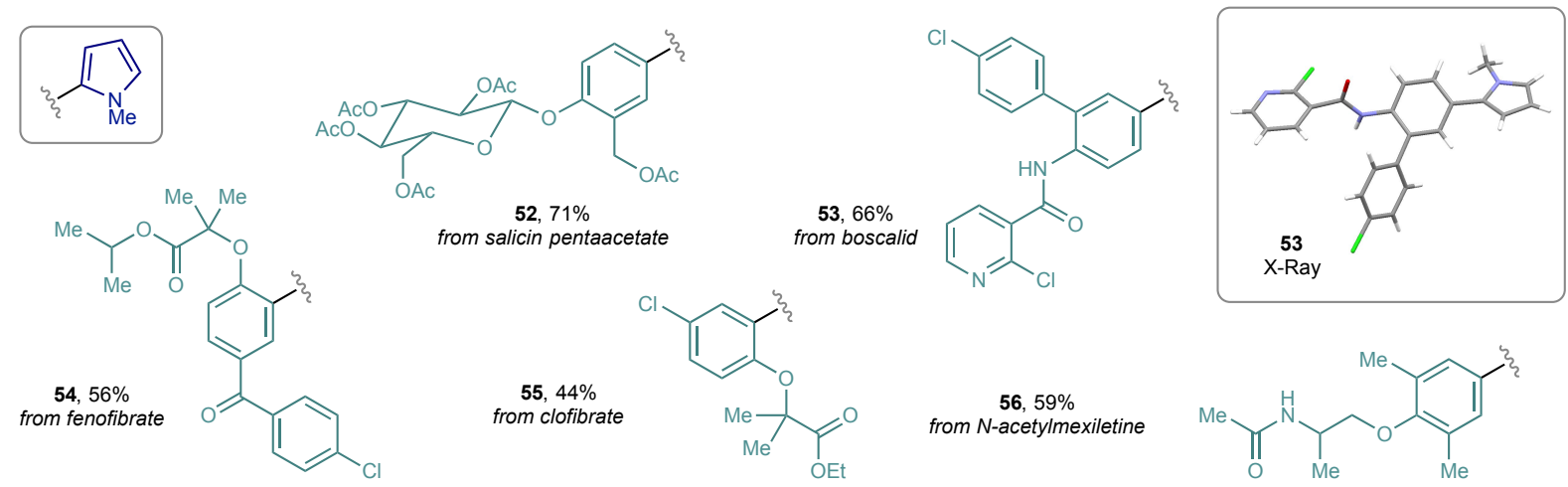

Figure 4. | Application of the one-pot, metal-free photoredox-catalyzed $\mathrm{C}-\mathrm{H} / \mathrm{C}-\mathrm{H}$ coupling of arenes, enabled by interrupted Pummerer activation. a, Concise synthesis of a bioactive pseudilin natural product and an analogue. Reaction conditions: A, arene (1.0 equiv.), DBTSO (2.0 equiv.), triflic anhydride (2.0 equiv.), $\mathrm{CH}_{2} \mathrm{Cl}_{2}(0.1$ $\mathrm{M}),-78{ }^{\circ} \mathrm{C}$ to $-40{ }^{\circ} \mathrm{C}, 2$ hour, then PTH (10 mol\%), $N$-Boc pyrrole (10 equiv.), DMSO (0.2 M), blue LED irradiation, 18 hours. B, Sodium methoxide ( $30 \% \mathrm{w} / \mathrm{w}$ in $\mathrm{MeOH}, 10$ equiv.), THF $(0.14 \mathrm{M}), 45^{\circ} \mathrm{C}, 2$ hours. C, chlorination: NCS (3.0 equiv.), $\mathrm{MeCN}(0.05 \mathrm{M}),-40{ }^{\circ} \mathrm{C}$ to RT, 15 hours. bromination: Pyridinium tribromide (3.0 equiv.), EtOH (0.06 M), RT, 30 minutes. D, Boron tribromide (1.1 equiv.), $\mathrm{CH}_{2} \mathrm{Cl}_{2}(0.04 \mathrm{M}),-78{ }^{\circ} \mathrm{C}$ to $0{ }^{\circ} \mathrm{C}, 2$ hours. b, Late-stage functionalization of bioactive molecules. Reaction conditions: arene (1.0 equiv.), DBTSO (1.1 equiv.), triflic anhydride (1.2 equiv.), $\mathrm{CH}_{2} \mathrm{Cl}_{2}(0.1 \mathrm{M}),-78{ }^{\circ} \mathrm{C}$ to RT, 2 hour, then PTH (10 mol\%), $N$-Methyl pyrrole (20 equiv.), DMSO (0.2 M), blue LED irradiation, 18 hours.

\section{Conclusions}


We have developed a one-pot strategy for the rapid construction of (hetero)biaryl motifs from nonprefunctionalized partners using a combination of organophotocatalysis and substrate activation using the interrupted Pummerer reaction. Our $\mathrm{C}-\mathrm{H} / \mathrm{C}-\mathrm{H}$ cross-coupling allows (hetero)biaryl carbon frameworks to be assembled under metal-free conditions, without multi-step synthesis. The marriage of the organic photoredox catalyst, 10-phenylphenothiazine, and intermediate aryldibenzothiophenium salts is unique and has led to a highly selective process that is broad in scope. The utility of the strategy has been exemplified by the synthesis of the bioactive natural product pentachloropseudilin and the modification of complex molecules of societal importance.

\section{Methods}

General Procedure for the interrupted Pummerer-enabled, photoredox-catalyzed C-H/C-H coupling of arenes. $\mathrm{Tf}_{2} \mathrm{O}$ (1.2 equiv.) was slowly added to a stirred solution of the arene $(0.2 \mathrm{mmol}, 1.0$ equiv.) and dibenzothiophene S-oxide (1.1 equiv.) in $\mathrm{CH}_{2} \mathrm{Cl}_{2}(0.1 \mathrm{M})$ at $-78^{\circ} \mathrm{C}$ under a nitrogen atmosphere. The resulting solution was stirred at this temperature for 15 minutes before warming to room temperature. After stirring for $1 \mathrm{~h}, \mathrm{TLC}$ analysis showed complete consumption of the arene starting material, at which point the solvent was removed in vacuo. 10Phenyl-phenothiazine (10 mol\%) was then added to the reaction vial, followed by evacuation and refilling with nitrogen (3 cycles). DMSO (0.2 M) was then added followed by the radical trap reagent. The reaction mixture was then irradiated with a Kessil $34 \mathrm{~W}$ blue LED lamp for $18 \mathrm{~h}$ before quenching with aqueous saturated $\mathrm{NaHCO}_{3}$ and dilution with EtOAc. The organic layer was washed with brine, dried using $\mathrm{MgSO}_{4}$, filtered, and concentrated in vacuo, to give the crude product, which was purified by column chromatography on silica gel.

\section{Data Availability}


All data is available in the main text or the Supplementary Information. Metrical parameters for the structures of $\mathbf{2}$ and $\mathbf{5 3}$ (see Supplementary Information) are available free of charge from the Cambridge Crystallographic Data Centre (https://www.ccdc.cam.ac.uk/) under reference numbers CCDC 1922367 and 1922368.

\section{References}

1 Johansson Seechurn, C. C. C., Kitching, M. O., Colacot, T. J. \& Snieckus, V. Palladium-Catalyzed CrossCoupling: A Historical Contextual Perspective to the 2010 Nobel Prize. Angew. Chem. Int. Ed. 51, 50625085 (2012).

2 Roughley, S. D. \& Jordan, A. M. The Medicinal Chemist's Toolbox: An Analysis of Reactions Used in the Pursuit of Drug Candidates. J. Med. Chem. 54, 3451-3479 (2011).

3 Brown, D. G. \& Boström, J. Analysis of Past and Present Synthetic Methodologies on Medicinal Chemistry: Where Have All the New Reactions Gone? J. Med. Chem. 59, 4443-4458 (2016).

4 Yang, Y., Lan, J., You, J. Chem. Rev. 117, 8787-8863 (2017)

5 Sambiagio, C., Schönbauer, D., Blieck, R., Dao-Huy, T., Pototschnig, G., Schaaf, P., Wiesinger, T., Zia, M. F., Wencel-Delord, J., Besset, T., Maes, B. U. W. \& Schnürch, M. A comprehensive overview of directing groups applied in metal-catalysed C-H functionalisation chemistry. Chem. Soc. Rev. 47, 6603-6743 (2018).

6 Hari, D. P., Schroll, P. \& König, B. Metal-Free, Visible-Light-Mediated Direct C-H Arylation of Heteroarenes with Aryl Diazonium Salts. J. Am. Chem. Soc. 134, 2958-2961 (2012).

7 Ghosh, I., Marzo, L., Das, A., Shaikh, R. \& König, B. Visible Light Mediated Photoredox Catalytic Arylation Reactions. Acc. Chem. Res. 49, 1566-1577 (2016).

8 Nguyen, J. D., D'Amato, E. M., Narayanam, J. M. R. \& Stephenson, C. R. J. Engaging unactivated alkyl, alkenyl and aryl iodides in visible-light-mediated free radical reactions. Nat. Chem. 4, 854-859 (2012).

9 Kim, H. \& Lee, C. Visible-Light-Induced Photocatalytic Reductive Transformations of Organohalides. Angew. Chem. Int. Ed. 51, 12303-12306 (2012). 
10 Ghosh, I., Ghosh, T., Bardagi, J. I. \& König, B. Reduction of aryl halides by consecutive visible light-induced electron transfer processes. Science 346, 725-728 (2014).

11 Marzo, L., Ghosh, I., Esteban, F. \& König, B. Metal-Free Photocatalyzed Cross Coupling of Bromoheteroarenes with Pyrroles. ACS Catal. 6, 6780-6784 (2016).

12 Ghosh, I. \& König, B. Chromoselective Photocatalysis: Controlled Bond Activation through Light-Color Regulation of Redox Potentials. Angew. Chem. Int. Ed. 55, 7676-7679 (2016).

13 Ghosh, I., Shaikh, R. S. \& König, B. Sensitization-Initiated Electron Transfer for Photoredox Catalysis. Angew. Chem. Int. Ed. 56, 8544-8549 (2017).

14 Smith, L. H. S., Coote, S. C., Sneddon, H. F. \& Procter, D. J. Beyond the Pummerer Reaction: Recent Developments in Thionium lon Chemistry. Angew. Chem. Int. Ed. 49, 5832-5844 (2010).

15 Eberhart, A. J., Imbriglio, J. E. \& Procter, D. J. Nucleophilic Ortho Allylation of Aryl and Heteroaryl Sulfoxides. Org. Lett. 13, 5882-5885 (2011).

16 Huang, X. \& Maulide, N. Sulfoxide-Mediated $\alpha$-Arylation of Carbonyl Compounds. J. Am. Chem. Soc. 133, 8510-8513 (2011).

17 Eberhart, A. J. \& Procter, D. J. Nucleophilic ortho-Propargylation of Aryl Sulfoxides: An Interrupted Pummerer/Allenyl Thio-Claisen Rearrangement Sequence. Angew. Chem. Int. Ed. 52, 4008-4011 (2013).

18 Huang, X., Patil, M., Farès, C., Thiel, W. \& Maulide, N. Sulfur(IV)-Mediated Transformations: From Ylide Transfer to Metal Free Arylation of Carbonyl Compounds. J. Am. Chem. Soc. 135, 7312-7323 (2013).

19 Peng, B., Huang, X., Xie, L-G. \& Maulide, N. A Brønsted Acid Catalyzed Redox Arylation. Angew. Chem. Int. Ed. 53, 8718-8721 (2014).

20 Peng, B., Geerdink, D., Fars, C. \& Maulide, N. Chemoselective Intermolecular $\alpha$-Arylation of Amides. Angew. Chem. Int. Ed. 53, 5462-5466 (2014).

21 Pulis, A. P. \& Procter, D. J. C-H Coupling Reactions Directed by Sulfoxides: Teaching an Old Functional Group New Tricks. Angew. Chem. Int. Ed. 55, 9842-9860 (2016).

22 Fernández-Salas, J. A., Eberhart, A. J. \& Procter, D. J. Metal-Free CH-CH-Type Cross-Coupling of Arenes and Alkynes Directed by a Multifunctional Sulfoxide Group. J. Am. Chem. Soc. 138, 790-793 (2016). 
23 Cowper, P., Jin, Y., Turton, M. D., Kociok-Köhn, G. \& Lewis, S. E. Azulenesulfonium Salts: Accessible, Stable, and Versatile Reagents for Cross-Coupling. Angew. Chem. Int. Ed. 55, 2564-2568 (2016).

24 Li, Y., Qiu, D., Gu, R., Wang, J., Shi, J. \& Li, Y. Aryne 1,2,3-Trifunctionalization with Aryl Allyl Sulfoxides. J. Am. Chem. Soc. 138, 10814-10817 (2016).

25 Yanagi, T., Otsuka, S., Kasuga, Y., Fujimoto, K., Murakami, K., Nogi, K., Yorimitsu, H. \& Osuka, A. MetalFree Approach to Biaryls from Phenols and Aryl Sulfoxides by Temporarily Sulfur-Tethered Regioselective C-H/C-H Coupling. J. Am. Chem. Soc. 138, 14582-14585 (2016).

26 Shrives, H. J., Fernández-Salas, J. A., Hedtke, C., Pulis, A. P. \& Procter, D. J. Regioselective synthesis of C3 alkylated and arylated benzothiophenes. Nat. Commun. 8, 14801 (2017).

27 Kaldre, D., Maryasin, B., Kaiser, D., Gajsek, O., González, L. \& Maulide, N. An Asymmetric Redox Arylation: Chirality Transfer from Sulfur to Carbon through a Sulfonium [3,3]-Sigmatropic Rearrangement. Angew. Chem. Int. Ed. 56, 2212-2215 (2017).

28 Chen, D., Feng, Q., Yang, Y., Cai, X-M., Wang F. \& Huang, S. Metal-free O-H/C-H difunctionalization of phenols by o-hydroxyarylsulfonium salts in water. Chem. Sci. 8, 1601-1606 (2017).

29 Yorimitsu, H. Cascades of Interrupted Pummerer Reaction-Sigmatropic Rearrangement. Chem. Rec. 17, 1156-1167 (2017).

30 Shang, L., Chang, Y., Luo, F., He, J-N., Huang, X., Zhang, L., Kong, L., Li, K. \& Peng, B. Redox-Neutral $\alpha-$ Arylation of Alkyl Nitriles with Aryl Sulfoxides: A Rapid Electrophilic Rearrangement. J. Am. Chem. Soc. 139, 4211-4217 (2017).

31 He, Z., Shrives, H. J., Fernández-Salas, J. A., Abengózar, A., Neufeld, J., Yang, K., Pulis, A. P. \& Procter, D. J. Synthesis of C2 Substituted Benzothiophenes via an Interrupted Pummerer/[3,3]-Sigmatropic/1,2Migration Cascade of Benzothiophene S-Oxides. Angew. Chem. Int. Ed. 57, 5759-5764 (2018).

32 Zhang, L., He, J-N., Liang, Y., Hu, M., Shang, L., Huang, X., Kong, L., Wang, Z-X. \& Peng, B. Selective [5,5]Sigmatropic Rearrangement by Assembly of Aryl Sulfoxides with Allyl Nitriles. Angew. Chem. Int. Ed. 58, 5316-5320 (2019). 
33 Berger, F., Plutschack, M. B., Riegger, J., Yu, W., Speicher, S., Ho, M., Frank, N. \& Ritter, T. Site-selective and versatile aromatic C-H functionalization by thianthrenation. Nature 567, 223-228 (2019).

$34 \mathrm{He}$, Z., Pulis, A. P. \& Procter, D. J. The Interrupted Pummerer Reaction in a Sulfoxide-Catalyzed Oxidative Coupling of 2-Naphthols. Angew. Chem. Int. Ed. 58, 7813-7817 (2019).

35 He, Z., Pulis, A. P., Perry, G. J. \& Procter, D. J. Pummerer chemistry of benzothiophene S-oxides: Metalfree alkylation and arylation of benzothiophenes, Phosphorus, Sulfur, and Silicon and the Related Elements (2019) DOI: 10.1080/10426507.2019.1602626

36 Šiaučiulis, M., Ahlsten, N., Pulis, A. P. \& Procter, D. J. Transition-Metal-Free Cross-Coupling of Benzothiophenes and Styrenes in a Stereoselective Synthesis of Substituted (E,Z)-1,3-Dienes. Angew. Chem. Int. Ed. 58, 8779-8783 (2019).

37 Aukland, M. H., Talbot, F. J. T., Fernández-Salas, J. A., Ball, M., Pulis, A. P. \& Procter, D. J. An Interrupted Pummerer/Nickel-Catalysed Cross-Coupling Sequence. Angew. Chem. Int. Ed. 57, 9785-9789 (2018).

38 Prier, C. K., Rankic, D. A. \& MacMillan, D. W. C. Visible Light Photoredox Catalysis with Transition Metal Complexes: Applications in Organic Synthesis. Chem. Rev. 113, 5322-5363 (2013).

39 Romero, N. A. \& Nicewicz, D. A. Organic Photoredox Catalysis. Chem. Rev. 116, 10075-10166 (2016).

40 Cismesia, M. A. \& Yoon, T. P. Characterizing chain processes in visible light photoredox catalysis. Chem. Sci. 6, 5426-5434 (2015).

41 Hedstrand, D. M., Kruizunga, W. H. \& Kellogg, R. M. Light induced and dye accelerated reductions of phenacyl onium salts by 1,4-dihydropyridines. Tetrahedron Lett. 14, 1255-1258 (1978).

42 Otsuka, S., Nogi, K., Rovis, T. \& Yorimitsu, H. Photoredox-Catalyzed Alkenylation of Benzylsulfonium Salts. Chem. Asian J. 14, 532-536 (2019).

43 Donck, S., Baroudi, A., Fensterbank, L., Goddard, J-P. \& Ollivier, C. Visible-Light Photocatalytic Reduction of Sulfonium Salts as a Source of Aryl Radicals. Adv. Synth. Catal. 355, 1477-1482 (2013).

44 Liu, W., Cao, H., Zhang, H., Zhang, H., Chung, K. H., He, C., Wang, H., Kwong, F. Y. \& Lei, A. Organocatalysis in Cross-Coupling: DMEDA-Catalyzed Direct C-H Arylation of Unactivated Benzene. J. Am. Chem. Soc. 132, 16737-16740 (2010). 
45 Discekici, E. H., Treat, N. J., Poelma, S. O., Mattson, K. M., Hudson, Z. M., Luo, Y., Hawker, C. J. \& De Alaniz, J. R. A highly reducing metal-free photoredox catalyst: design and application in radical dehalogenations. Chem. Commun. 51, 11705-11708 (2015).

46 Garrido-Castro, A. F., Salaverri, N., Maestro, M. C. \& Alemán, J. Intramolecular Homolytic Substitution Enabled by Photoredox Catalysis: Sulfur, Phosphorus, and Silicon Heterocycle Synthesis from Aryl Halides. Org. Lett. (2019) DOI: 10.1021/acs.orglett.9b01911

47 Wang, H. \& Jui, N. T. Catalytic Defluoroalkylation of Trifluoromethylaromatics with Unactivated Alkenes. J. Am. Chem. Soc. 140, 163-166 (2018).

48 Kunfermann, A., Witschel, M., Illarionov, B., Martin, R., Rottmann, M., Höffken, H. W., Seet, M., Eisenreich, W., Knölker, H-J., Fischer, M., Bacher, A., Groll, M. \& Diederich, F. Pseudilins: Halogenated, Allosteric Inhibitors of the Non-Mevalonate Pathway Enzyme IspD. Angew. Chem. Int. Ed. 53, 2235-2239 (2014).

49 Preller, M., Chinthalapudi, K., Martin, R., Knölker, H-J. \& Manstein, D. J. Inhibition of Myosin ATPase Activity by Halogenated Pseudilins: A Structure - Activity Study. J. Med. Chem. 54, 3675-3685 (2011).

50 Martin, R., Jäger, A., Böhl, M., Richter, S., Fedorov, R., Manstein, D. J., Gutzeit, H. O. \& Knölker, H-J. Total Synthesis of Pentabromo- and Pentachloropseudilin, and Synthetic Analogues-Allosteric Inhibitors of Myosin ATPase. Angew. Chem. Int. Ed. 48, 8042-8046 (2009).

Supplementary Information is available in the online version of the paper.

Acknowledgements We thank the EPSRC (Doctoral Prize Fellowship to M.H.A.; Established Career Fellowship to D.J.P.) and The University of Manchester (Lectureship to G.J.P.P.; Studentship to M.Š.) for their generous support. Additional thanks go to Dr Louise Natrajan and Dr Fabio Julia Hernandez for their assistance with the fluorescence quenching and electrochemical studies, and to Dr Daniele Leonori for his advice on the design of experiments. 
Author Contributions M.H.A. and D.J.P. conceived the study and co-wrote the manuscript. M.H.A. designed and performed experiments and M.S., A.W. and G.P. performed experiments.

Author Information Reprints and permissions information is available at www.nature.com/reprints. The authors declare no competing financial interests. Readers are welcome to comment on the online version of this article. Correspondence and requests for materials should be addressed to D. J. P. (david.j.procter@manchester.ac.uk). 


\section{Metal-Free Photoredox-catalyzed C-H/C-H Coupling of Arenes Enabled by Interrupted Pummerer Activation}

Miles H. Aukland, ${ }^{1}$ Mindaugas Šiaučiulis, ${ }^{1}$ Adam West, ${ }^{1}$ Gregory J. P. Perry, ${ }^{1}$ and David J. Procter* ${ }^{1}$

${ }^{1}$ School of Chemistry, University of Manchester, Oxford Road, Manchester, M13 9PL, UK.

\section{CONTENTS}

General Information

Optimisation of the Interrupted Pummerer-Enabled, Photoredox-Catalyzed C-H/C-H Coupling of Arenes

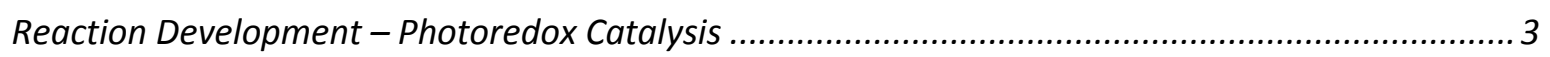

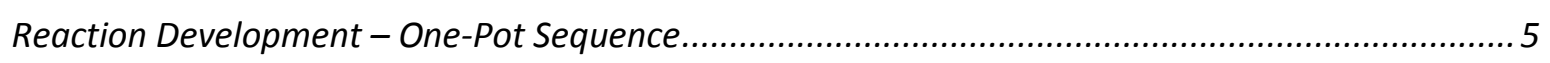

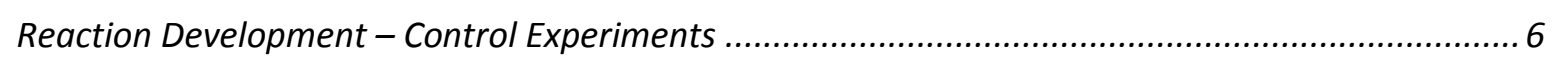

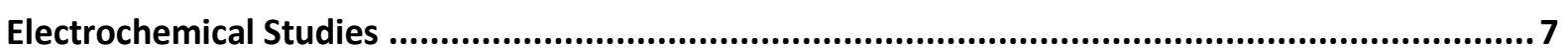

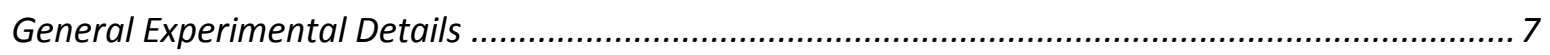

Electrochemical Potentials \& Cyclic Voltammograms ................................................................ 7

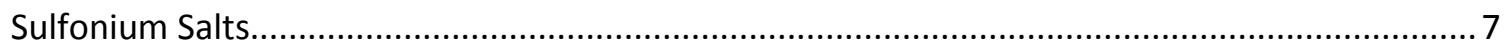

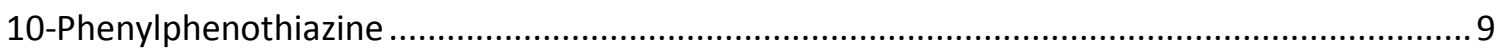

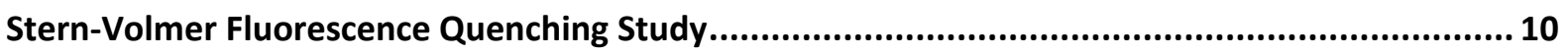

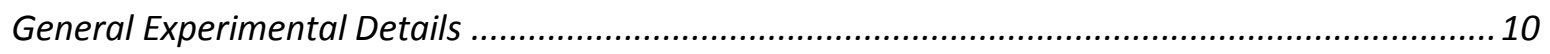

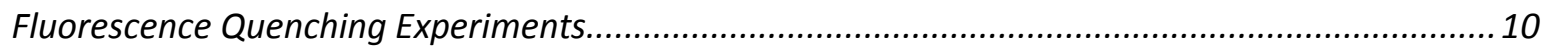

Synthetic Procedures \& Compound Characterisation .................................................................... 14

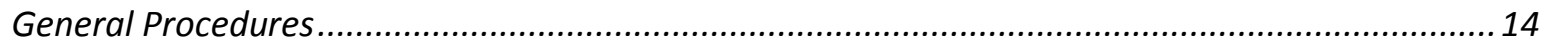

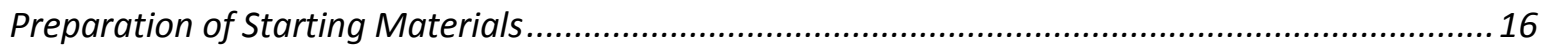

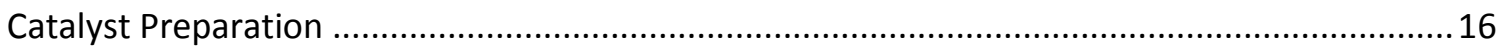

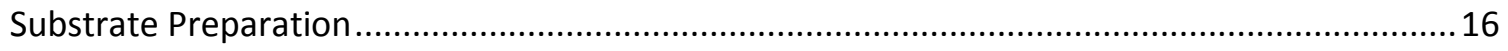

Interrupted Pummerer-Enabled, Photoredox-Catalyzed C-H/C-H Coupling of Arenes.....................22

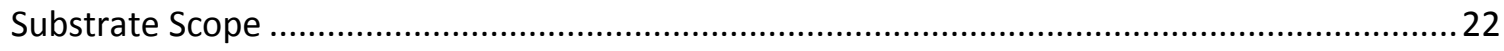

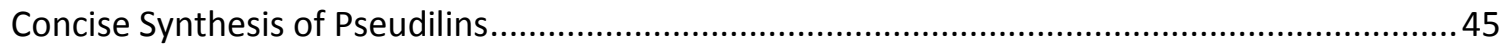

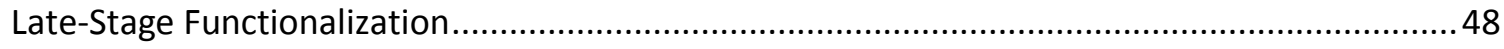

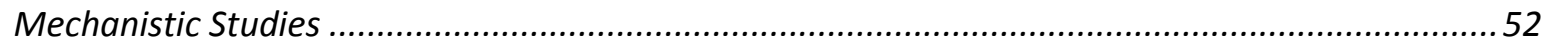

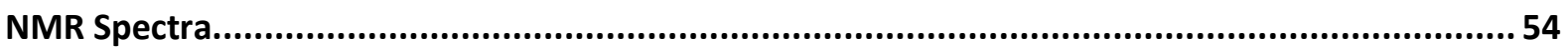

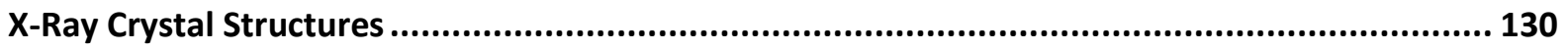

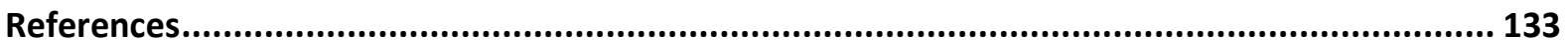




\section{GENERAL INFORMATION}

\section{General Experimental Information}

Glassware for inert atmosphere reactions was oven-dried and cooled under a flow of nitrogen. THF was distilled over sodium wire and benzophenone. All other solvents and reagents were purchased from commercial sources and used as supplied. Photoredox reactions were subjected to irradiation from a 34W Kessil blue LED bulb, with the reaction tube placed approximately $10 \mathrm{~cm}$ from the bulb. Routine TLC analysis was carried out on aluminium sheets coated with silica gel, $0.20 \mathrm{~mm}$ (Macherey-Nagel, Polygram ${ }^{\circledR}$ Sil G/UV254). TLC plates were visualized by UV absorption, potassium permanganate, $p$-anisaldehyde, or phosphomolybdic acid solution and heating.

\section{General Analytical Information}

Novel compounds were characterized by NMR, IR spectroscopy, HRMS, and melting point. ${ }^{1} \mathrm{H}-\mathrm{NMR}$ spectra were obtained on a 400 or $500 \mathrm{MHz}$ Bruker spectrometer. ${ }^{13} \mathrm{C}$-NMR spectra were obtained at 100 or $125 \mathrm{MHz}$. All NMR spectra were processed using MestReNova@ NMR software. All chemical shift values are reported in parts per million (ppm) relative to residual protio solvent and were determined in either $\mathrm{CDCl}_{3}, \mathrm{CD}_{3} \mathrm{CN}$, or $\mathrm{D}_{6}$-DMSO with coupling constant $(J)$ values reported in $\mathrm{Hz}$. The notation of signals is - Proton: $\delta$ chemical shift in ppm (number of protons, multiplicity, $J$ value(s), proton assignment). Carbon: $\delta$ chemical shift in $\mathrm{ppm}$ (carbon assignment). If assignment is ambiguous, for example in the case of overlapping signals, a shift range is reported. Low-resolution and high-resolution mass spectra were obtained using either positive and/or negative electrospray ionisation (ES), or atmospheric-pressure chemical ionisation (APCI) techniques. IR spectra were recorded on an ATR FTIR spectrometer using neat samples. Melting points were measured on solids obtained after chromatography. 


\section{OPTIMISATION OF THE INTERRUPTED PUMMERER-ENABLED, PHOTOREDOX- CATALYZED C-H/C-H COUPLING OF ARENES}

All optimization reactions were carried out on $0.2 \mathrm{mmol}$ scale. The crude reaction mixtures were analyzed by ${ }^{1} \mathrm{H}-\mathrm{NMR}$ with $\mathrm{MeNO}_{2}$ as an internal standard.

\section{Reaction Development - Photoredox Catalysis}

Optimization of the visible-light-mediated direct arylation step was performed using dibenzothiophenium salt, 2. As outlined in Table S1, the (hetero)biaryl, $\mathbf{S 1}$ was formed in low yield from the reaction of $\mathbf{2}$ and furan in the presence of typical transition-metal photoredox catalysts in DMSO solvent (Entries 1 \& 2). Various common organic photoredox catalysts were screened and also showed poor catalytic activity (Entries 3-7). The use of 10-phenyl phenothiazine (PTH) as the photoredox catalyst had a significant effect, providing a more efficient reaction (Entry 8). Exploring other polar solvents (required to solubilise the dibenzothiophenium salt, 2) for the reaction showed that the chemistry is optimal when using DMSO. Increasing the amount of the furan coupling partner gave increasing yields of the desired product (Entries $17 \& 18$ ), however, as a compromise between reaction efficiency and reactant stoichiometry, 20 equivalents of radical trap was used going forward. Catalyst loading of $10 \mathrm{~mol} \%$ was found to be optimal, as when increasing or decreasing the catalyst quantity, the yield of S1 decreased (Entries 19 \& 20). Finally, dibenzothiophenium salts proved crucial as when substituting $\mathbf{2}$ for the tetrahydrothiophenium salt $\mathbf{S 4}$, none of the desired product was detected (Entry 21). 
Table S1. Optimization of the Reaction Conditions
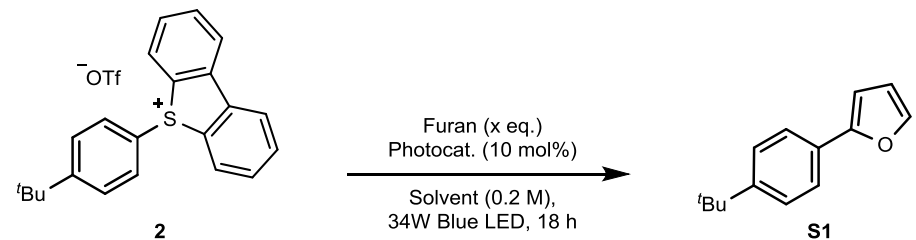

\begin{tabular}{|c|c|c|c|c|}
\hline Entry & Photocatalyst & Solvent & $x(e q)$ & Yield (\%) \\
\hline 1 & $\operatorname{Ir}(p p y)_{3}$ & DMSO & 10 & 34 \\
\hline 2 & $\mathrm{Ru}(\mathrm{bpy})_{3} \mathrm{Cl}_{2}$ & DMSO & 10 & 19 \\
\hline 3 & Eosin $Y$ & DMSO & 10 & 5 \\
\hline 4 & $\mathrm{Acr}-\mathrm{BF}_{4}$ & DMSO & 10 & 10 \\
\hline 5 & Rhodamine B & DMSO & 10 & N.D. \\
\hline 6 & Fluorescein & DMSO & 10 & 15 \\
\hline 7 & TXO & DMSO & 10 & 30 \\
\hline 8 & PTH & DMSO & 10 & 51 \\
\hline 9 & MTH & DMSO & 10 & 37 \\
\hline 10 & PTH & NMP & 10 & 8 \\
\hline 11 & PTH & DMF & 10 & 20 \\
\hline 12 & PTH & DMA & 10 & 24 \\
\hline 13 & PTH & $\mathrm{MeCN}$ & 10 & N.D. \\
\hline 14 & PTH & $\mathrm{MeOH}$ & 10 & N.D. \\
\hline 15 & PTH & Acetone & 10 & N.D. \\
\hline 16 & PTH & $\mathrm{CH}_{2} \mathrm{Cl}_{2}$ & 10 & N.D. \\
\hline 17 & PTH & DMSO & 20 & $63(58)^{\mathrm{e}}$ \\
\hline 18 & PTH & DMSO & 69 & 76 \\
\hline $19^{b}$ & PTH & DMSO & 20 & 35 \\
\hline $20^{c}$ & PTH & DMSO & 20 & 46 \\
\hline $21^{d}$ & PTH & DMSO & 20 & N.D. \\
\hline
\end{tabular}

${ }^{a}$ Determined by ${ }^{1} \mathrm{H}$ NMR using a $\mathrm{MeNO}_{2}$ internal standard. ${ }^{b} 5$ mol\% PTH added. ${ }^{\mathrm{c}} 20 \mathrm{~mol} \% \mathrm{PTH}$ added.

${ }^{\mathrm{d}}$ Tetrahydrothiophenium salt $\mathbf{S} \mathbf{4}$ was used in place of $\mathbf{2}^{\mathrm{e}}$ Isolated yield.

Acr $=9$-mesityl-10-methylacridinium. TXO = thioxanthen-9-one. PTH = 10-phenyl-phenothiazine. MTH = 10-methylphenothiazine. 


\section{Reaction Development - One-Pot Sequence}

A short optimization was necessary to facilitate the one-pot process (Table S2). Dichloromethane is the optimal solvent for the interrupted Pummerer (IP) chemistry, however, from previous optimization studies (vida supra), dichloromethane was an unsatisfactory solvent for the direct arylation step. Addition of a solution of the radical trap and PTH to the reaction mixture after sulfenylation provided the desired product, but in low yield (Entry 1). Removing the dichloromethane solvent in vacuo before adding the components for the second step delivered the desired product $\mathbf{S 1}$ in comparable yield to that obtained starting from dibenzothiophenium salt $\mathbf{2}$ (Entry 2). One equivalent of triflic acid is generated in the IP activation step and we hypothesized that the presence of acid may lead to degradation of the heterocyclic radical trap and/or the coupling product. However, the use of two equivalents of anhydrous potassium carbonate gave a lower yield (Entry 3).

Table S2. Optimization of the One-Pot Sequence

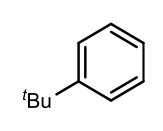

1

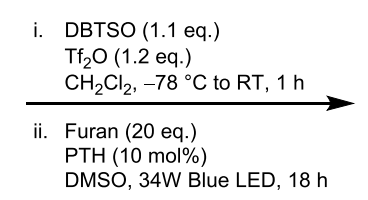

i. DBTSO (1.1 eq.

$\mathrm{CH}_{2} \mathrm{Cl}_{2},-78{ }^{\circ} \mathrm{C}$ to $\mathrm{RT}, 1 \mathrm{~h}$

Furan (20 eq.

DMSO, 34W Blue LED, 18

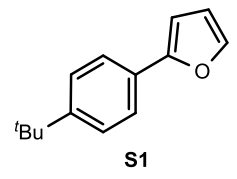

Notes

Direct addition

Solvent swap

60

Solvent swap

${ }^{\text {a }}$ Determined by ${ }^{1} \mathrm{H}$ NMR using a $\mathrm{MeNO}_{2}$ internal standard. ${ }^{\mathrm{b}} 2$ eq. $\mathrm{K}_{2} \mathrm{CO}_{3}$ added. 


\section{Reaction Development - Control Experiments}

Control experiments were conducted (Table S3). When performing the reaction in the absence of PTH a small amount of the arylated product is formed, presumably from direct excitation of the dibenzothiophenium salt 2 (Entry 1). Running the reaction in the dark led to complete recovery of the dibenzothiophenium salt $\mathbf{2}$ (Entry 2). When the reaction was carried out open to air, the yield of the product $\mathbf{S 1}$ dropped significantly, (Entry 3). Finally, the addition of water to the reaction medium had little effect on the yield (Entry 4).

Table S3. Control Experiments
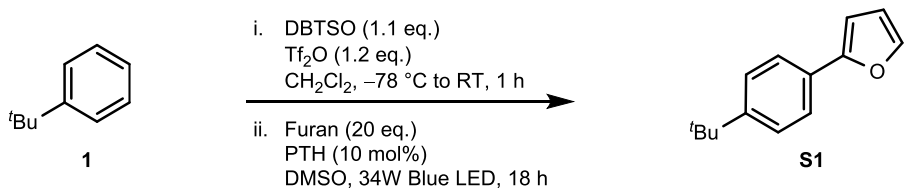

\begin{tabular}{ccc}
\hline Entry & Yield(\%) $^{\mathbf{a}}$ & Notes \\
\hline 1 & $9(91$ salt $)$ & Without PTH \\
2 & $>95$ salt & Without light \\
3 & $21(36$ salt $)$ & Open to air \\
4 & 58 & 1 eq. $\mathrm{H}_{2} \mathrm{O}$ added \\
\hline
\end{tabular}

${ }^{\mathrm{a}}$ Determined by ${ }^{1} \mathrm{H}$ NMR using a $\mathrm{MeNO}_{2}$ internal standard. 


\section{ELECTROCHEMICAL STUDIES}

\section{General Experimental Details}

Cyclic voltammetry was conducted on an EmStat (PalmSens) potentiostat using a 3-electrode cell configuration. A glassy carbon working electrode was employed alongside a platinum wire counter electrode and a $\mathrm{Ag} / \mathrm{AgCl}$ reference electrode. All the solutions were degassed by bubbling $\mathrm{N}_{2}$ prior to measurements. $5 \mathrm{mM}$ solutions of the desired compounds were freshly prepared in dry DMSO along with $0.1 \mathrm{M}$ of tetrabutylammonium hexafluorophosphate as supporting electrolyte and were examined at a scan rate of $0.1 \mathrm{~V} \mathrm{~s}^{-1}$. Ferrocene $\left(E_{1 / 2}=+0.435 \mathrm{~V} \text { vs SCE }\right)^{1}$ was added at the end of the measurements as an internal standard to determine the precise potential scale. Potential values are given versus the saturated calomel electrode (SCE). Irreversible reduction waves were obtained in all cases; therefore, the reduction potentials were obtained from the maximum, $E_{p} m a x$.

\section{Electrochemical Potentials \& Cyclic Voltammograms}

Electrochemical studies were conducted to identify the reduction potentials of the dibenzothiophenium salts. Attempts to determine the reduction potentials in acetonitrile gave inconsistent and non-reproducible results, likely due to decomposition on the electrode surface. As a result, reduction potentials were determined in DMSO. The excited state reduction potential of PTH was also determined in DMSO.

\section{Sulfonium Salts}

Figure S1. Cyclic voltammogram of 2

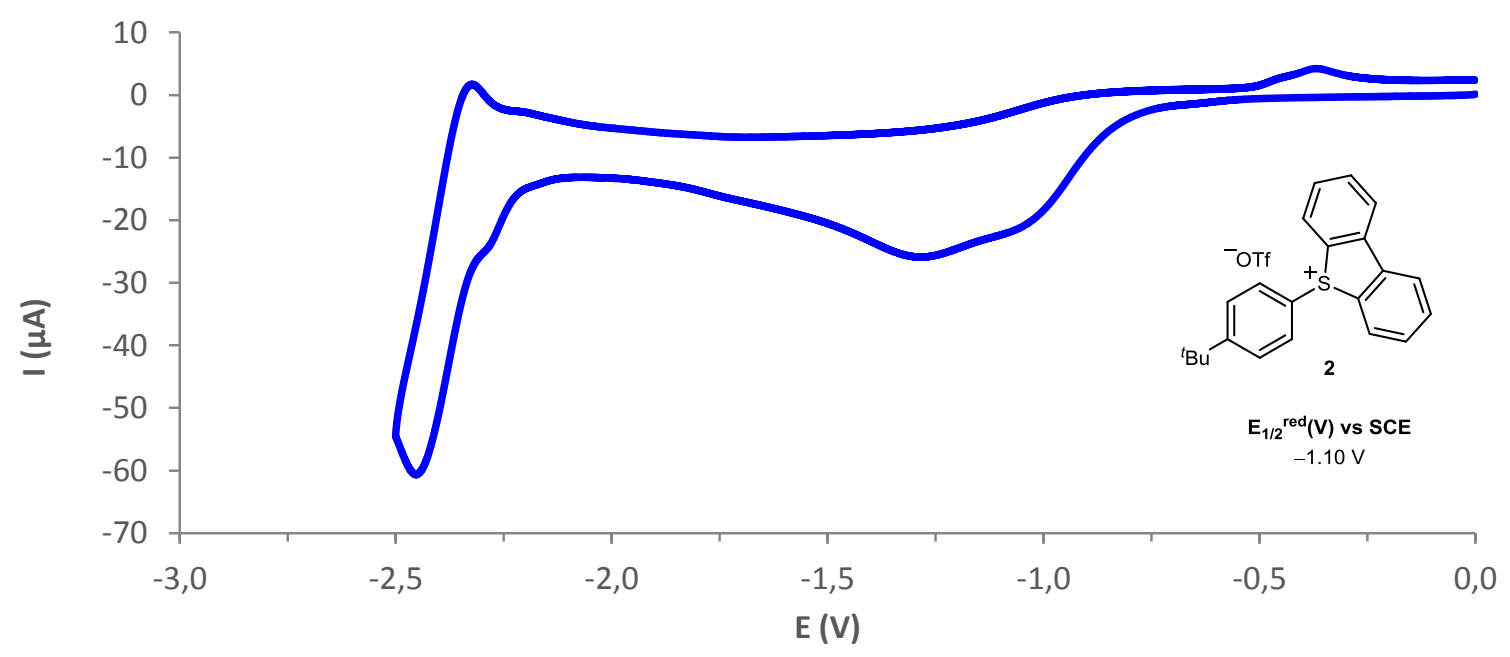


Figure S2. Cyclic voltammogram of S2

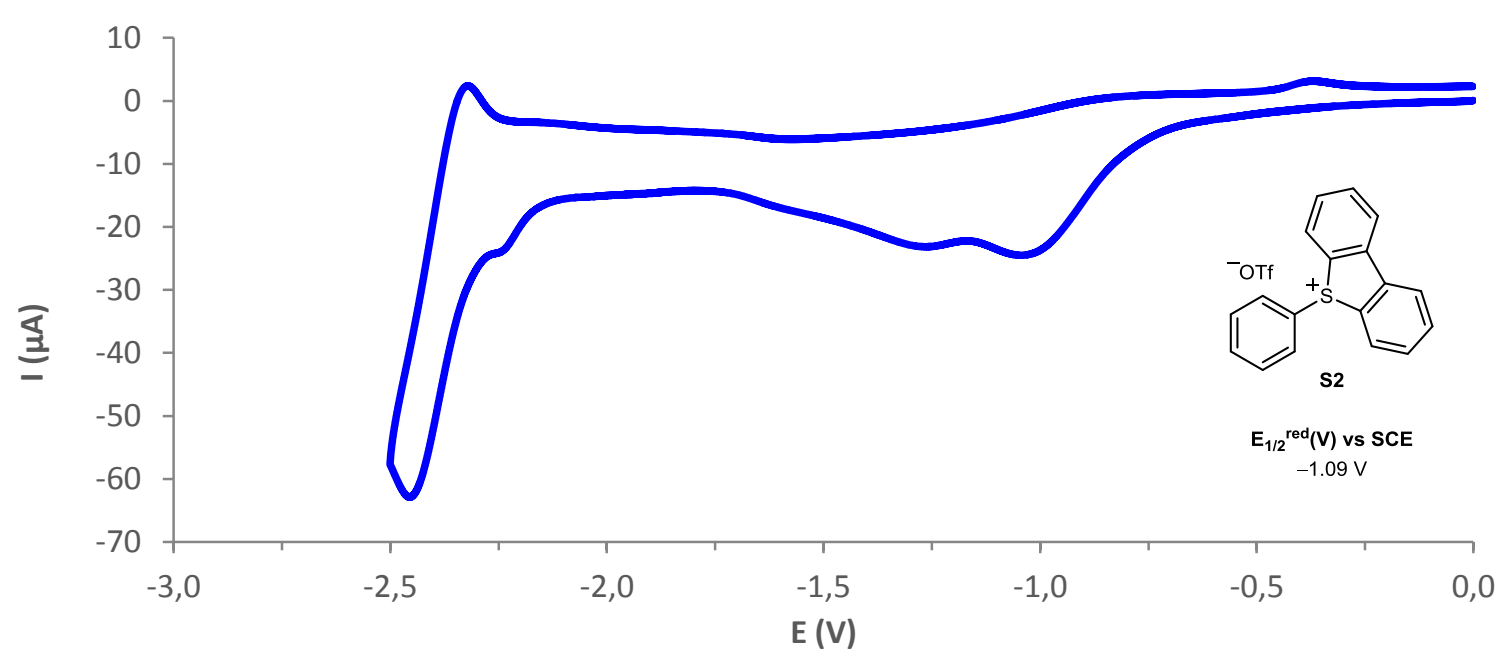

Figure S3. Cyclic voltammogram of S3

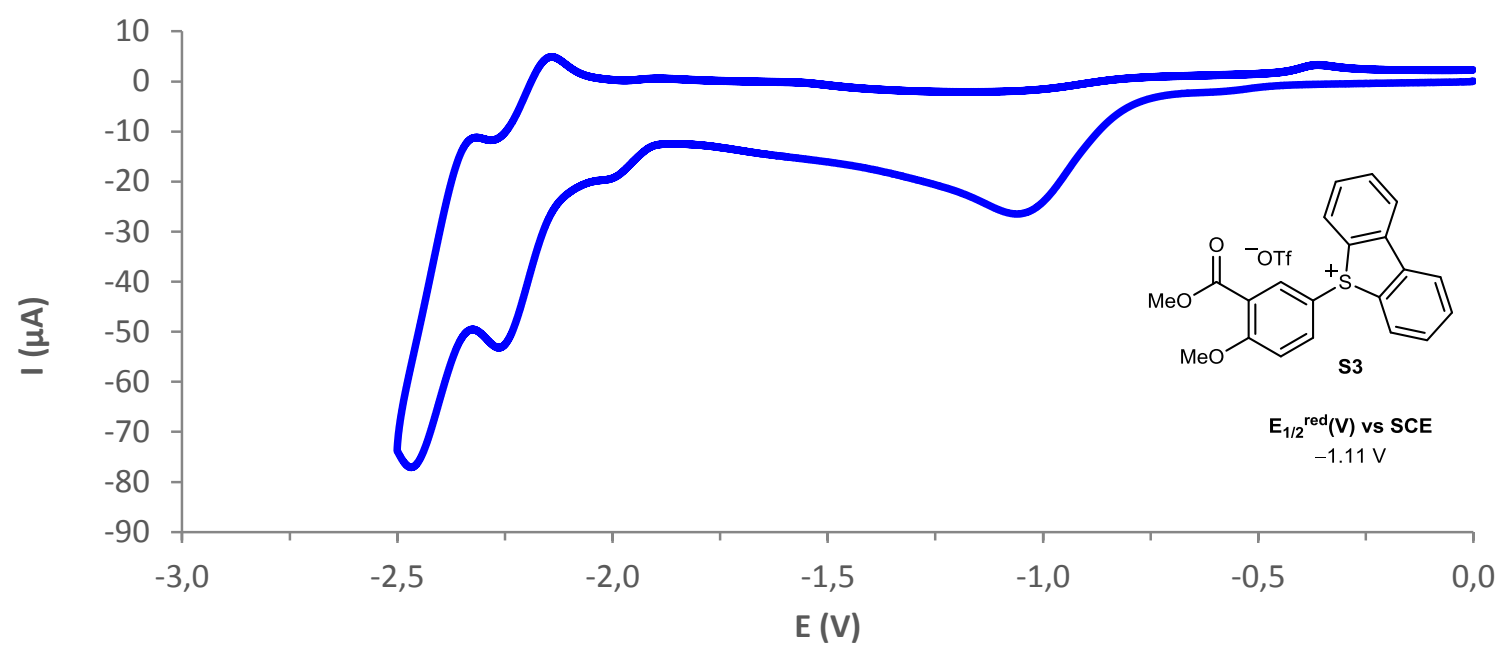

Figure S4. Cyclic voltammogram of S4

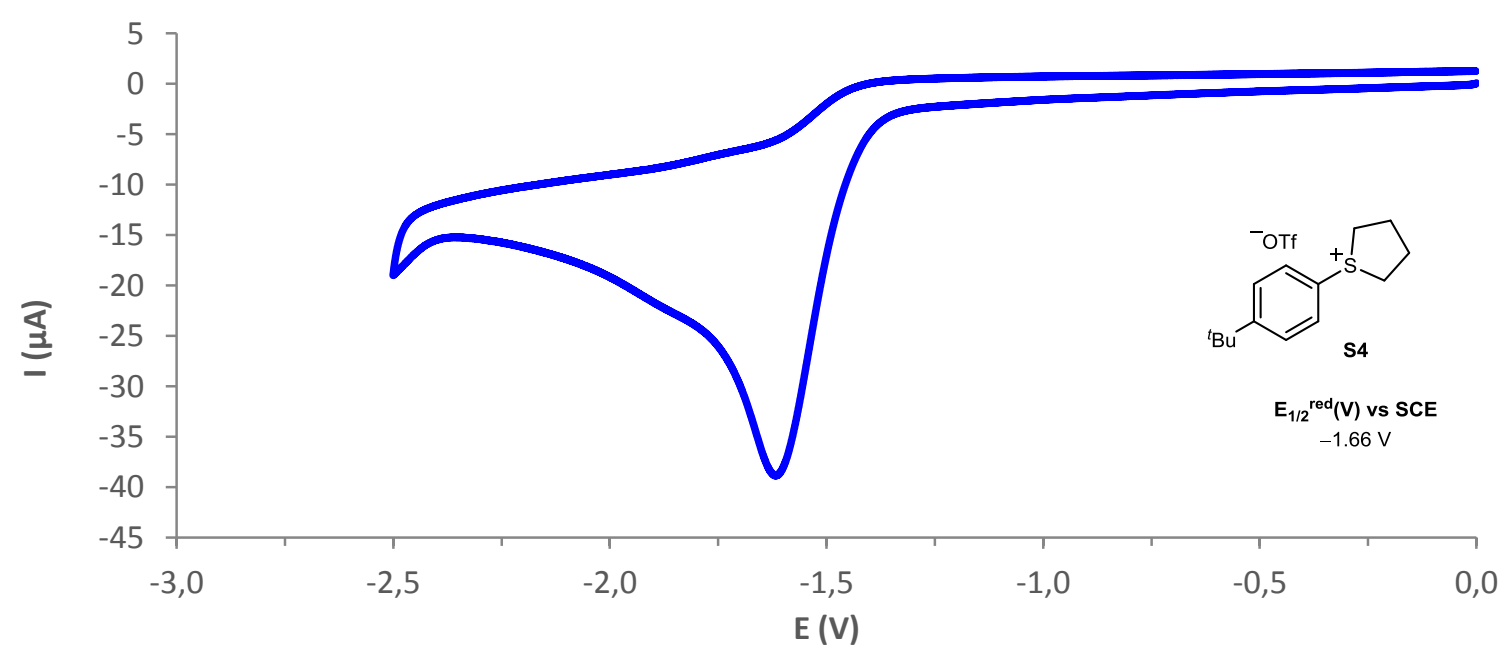


Figure S5. Cyclic voltammogram of PTH

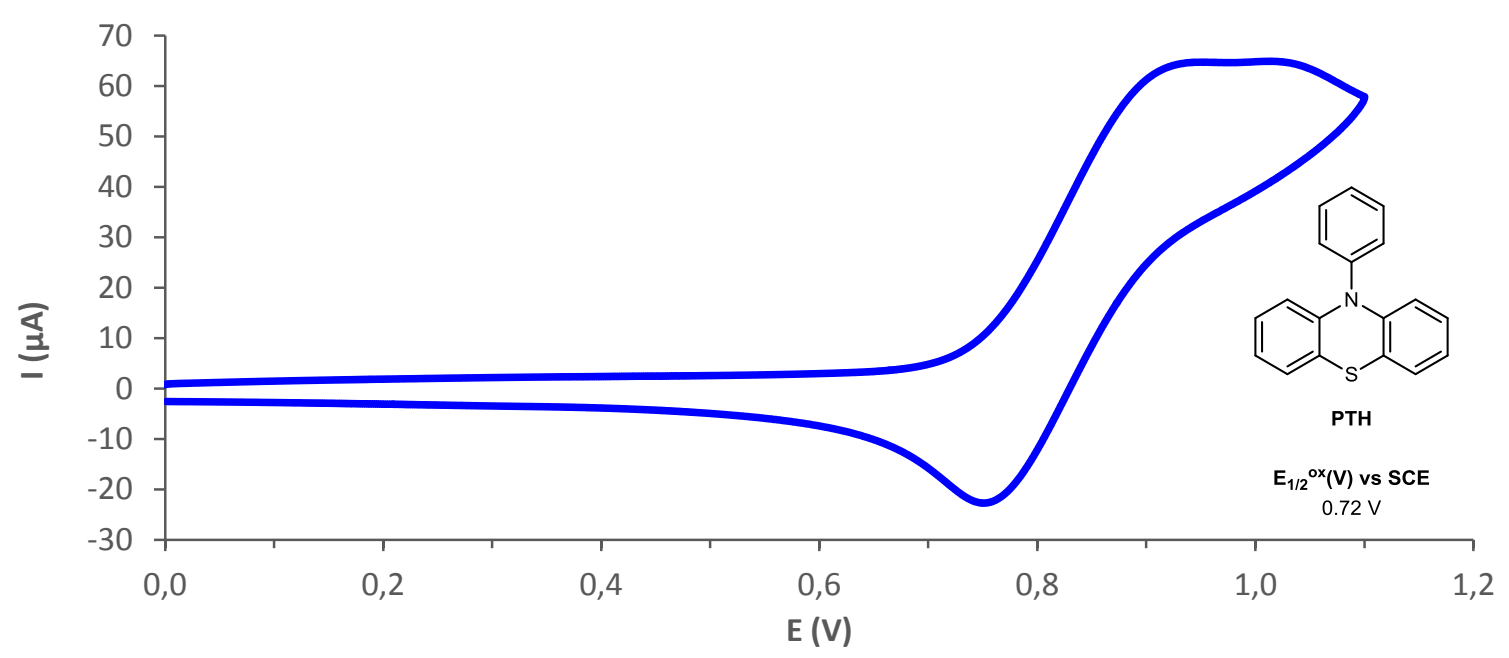

Figure S6. Normalized emission spectrum of PTH in DMSO (0.17 mM)

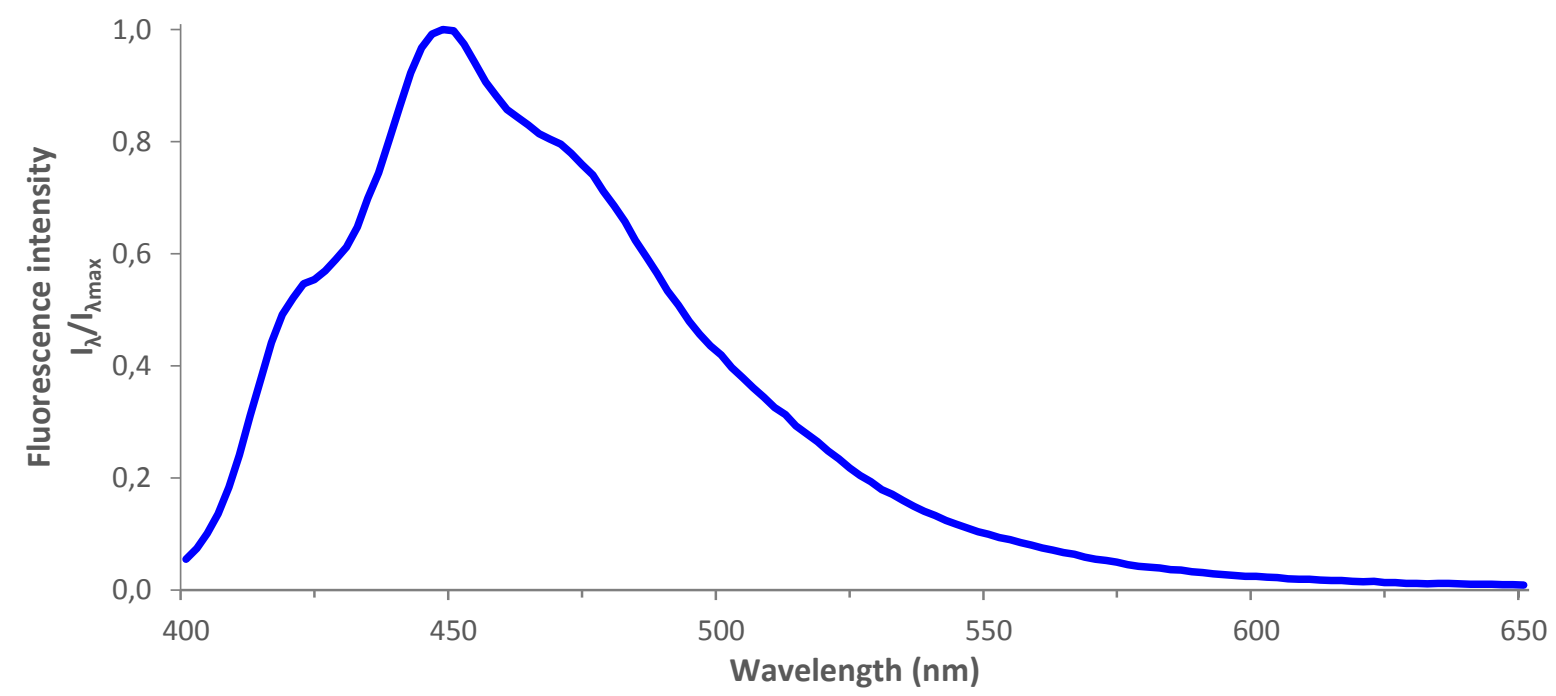

The photoluminescence maximum was estimated to be $448 \mathrm{~nm}$ (Figure S6). Using the photoluminescence maximum and $\mathrm{E}_{1 / 2}{ }^{\mathrm{ox}}$, the excited state reduction potential was determined for $\operatorname{PTH}\left(\mathrm{E}_{1 / 2}\left(\mathrm{PTH}^{+*} / \mathrm{PTH}^{*}\right)=-2.05 \mathrm{~V}\right)$ according to the following equations:

$$
E_{1 / 2}\left(P T H^{+\cdot} / P T H^{*}\right)=E^{o x}-E_{0,0}
$$

where $E_{0,0}=h c /_{\lambda}=1240 \mathrm{~nm} / \lambda_{\max }$ 


\section{STERN-VOLMER FLUORESCENCE QUENCHING STUDY}

\section{General Experimental Details}

Emission spectra were recorded on an Edinburgh Instrument FP920 Phosphorescence Lifetime Spectrometer equipped with a 450 watt xenon lamp (with single $300 \mathrm{~mm}$ focal length excitation and emission monochromators in Czerny Turner configuration) and a red sensitive photomultiplier in a Peltier (air-cooled) housing, (Hamamatsu R928P). All spectra are an average of three spectra and the same excitation and emission slits were used throughout. All spectra were corrected for the excitation source and the detector response using the correction files provided in the software.

\section{Fluorescence Quenching Experiments}

To evaluate the role of PTH in the process, we performed Stern-Volmer fluorescence quenching experiments, according to the procedure described be Read De Alaniz ${ }^{2}$ and Jui ${ }^{3}$. The quenching of PTH was conducted in DMSO, with a catalyst concentration of $0.17 \mathrm{mM}$ in a quartz cell with a $1 \mathrm{~cm}$ path length and an excitation wavelength of $350 \mathrm{~nm}$ used. The emission of PTH was measured with varying amounts of dibenzothiophenium salt, 2 (0.24, 0.50, \& $2.0 \mathrm{M}$ solutions in DMSO), furan (1.0 M in DMSO) and neat pyrrole to minimise the total volume change. All spectra were corrected for small changes in volume upon addition of the substrates. As demonstrated in Figure S7, the emission intensity decreased with increasing amounts of $\mathbf{2}$, providing evidence for the direct substrate reduction by photoexcited PTH $\left(\mathrm{PTH}^{*}\right)$. No significant quenching was observed in similar experiments using the radical traps $N$-methylpyrrole (Figure S9), whereas furan exhibited a small degree of quenching (Figure S11). 


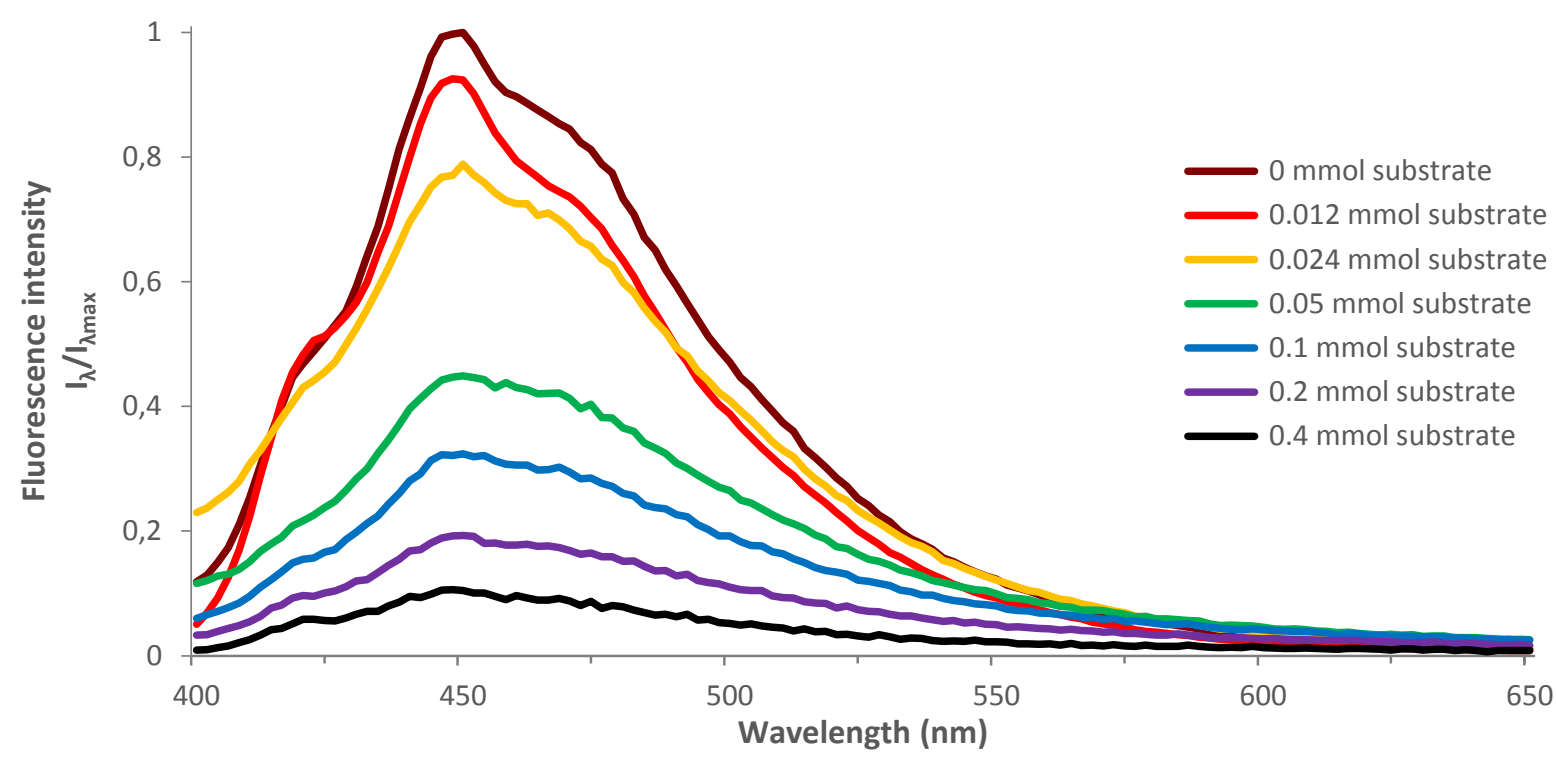

Figure S7. Emission spectra of PTH in DMSO $(0.17 \mathrm{mM})$ with varying concentrations of dibenzothiophenium salt, 2, normalized to PTH with no dibenzothiophenium salt added.

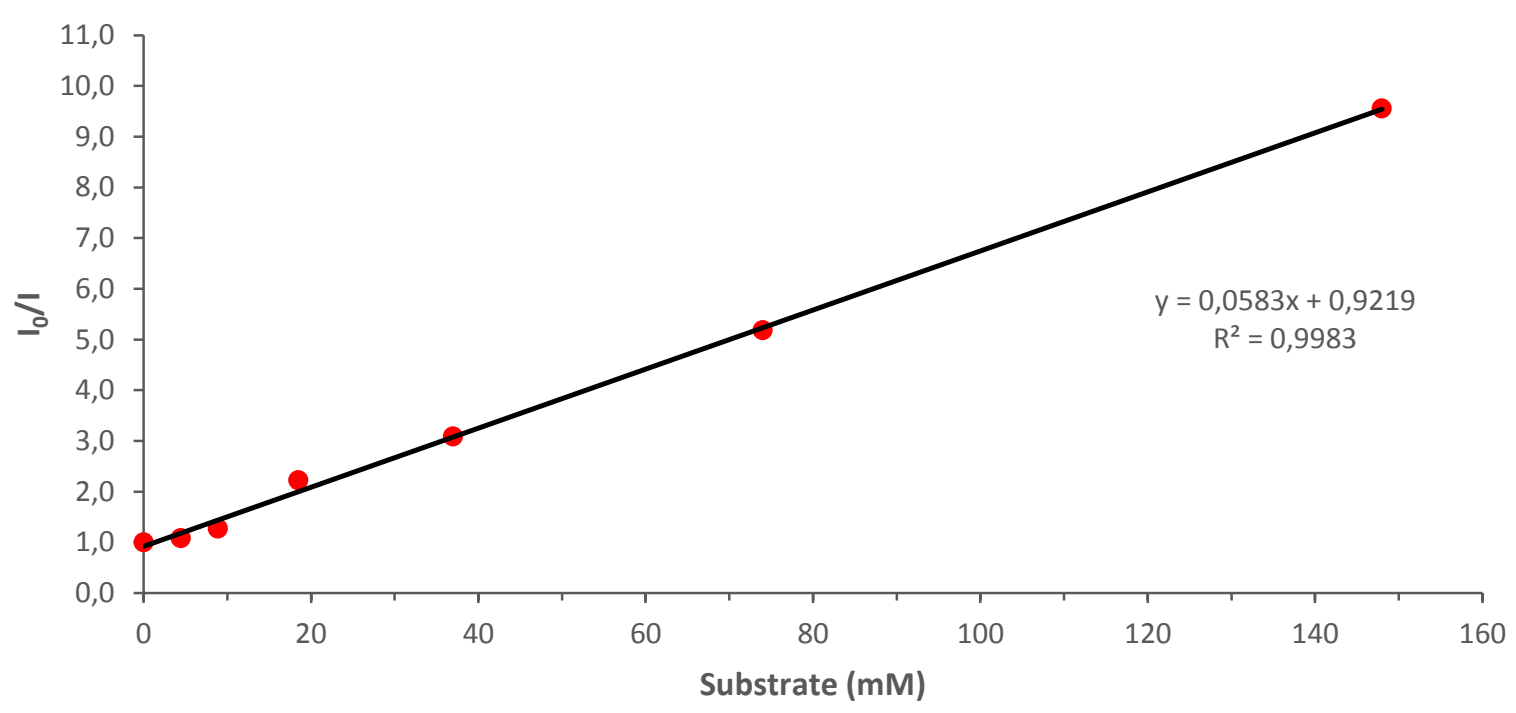

Figure S8. Stern-Volmer plot for PTH in DMSO (0.17 mM) with varying dibenzothiophenium salt, 2.

$\mathrm{KSV}=58.3$ 


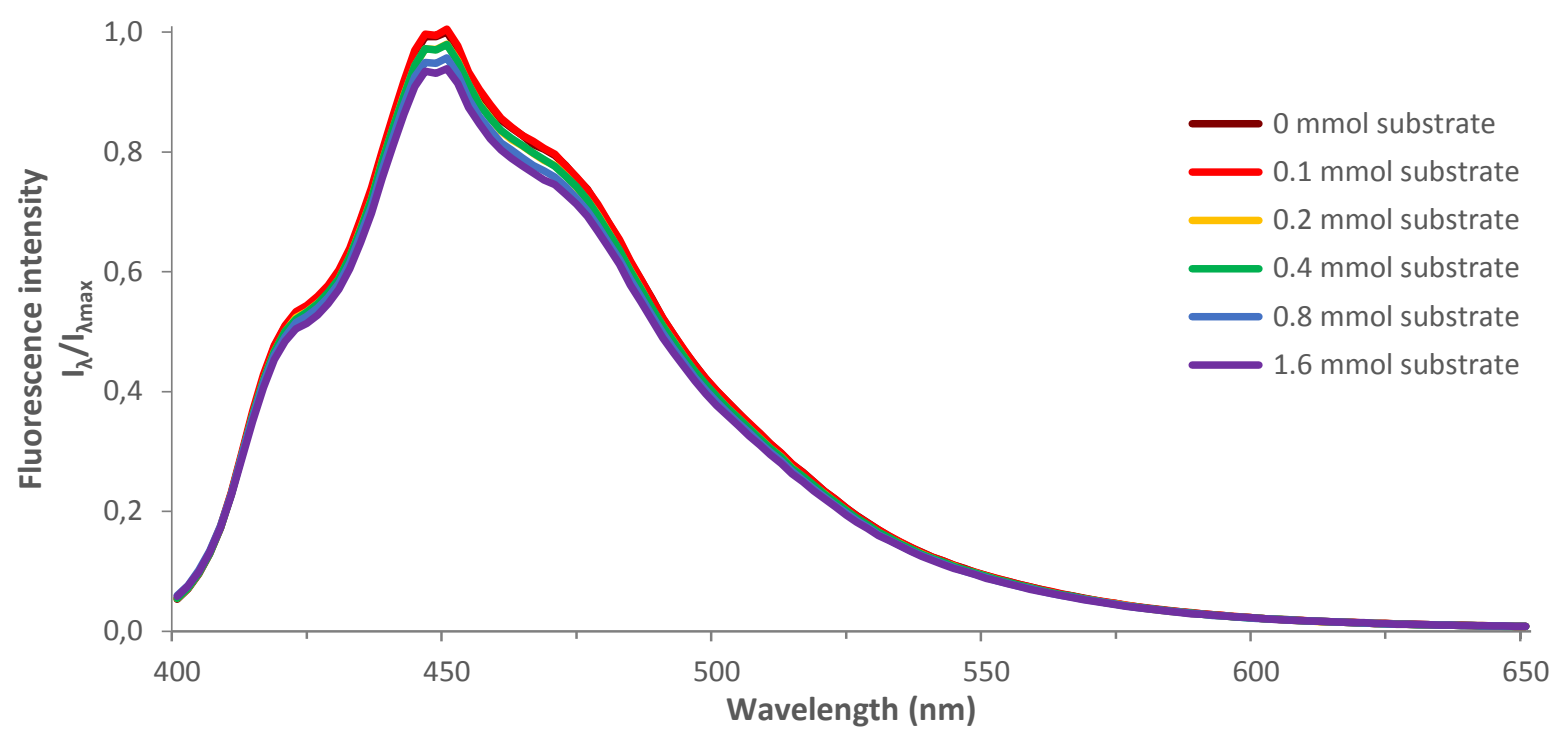

Figure 59. Emission spectra of PTH in DMSO $(0.17 \mathrm{mM})$ with varying concentrations of $N$-methylpyrrole, normalized to PTH with no $\mathrm{N}$-methylpyrrole added.

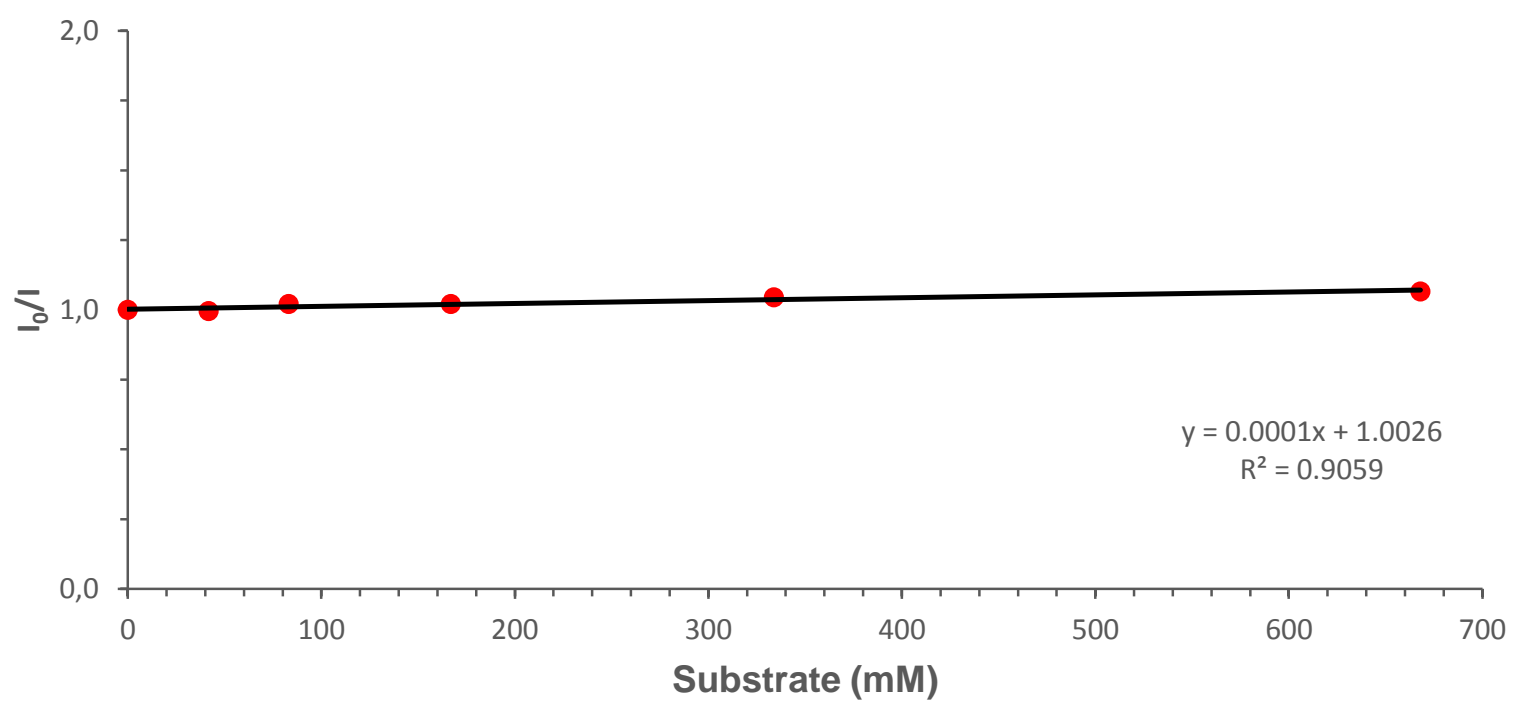

Figure S10. Stern-Volmer plot for PTH in DMSO $(0.17 \mathrm{mM})$ with varying $N$-methylpyrrole.

KSv $=0.10$ 


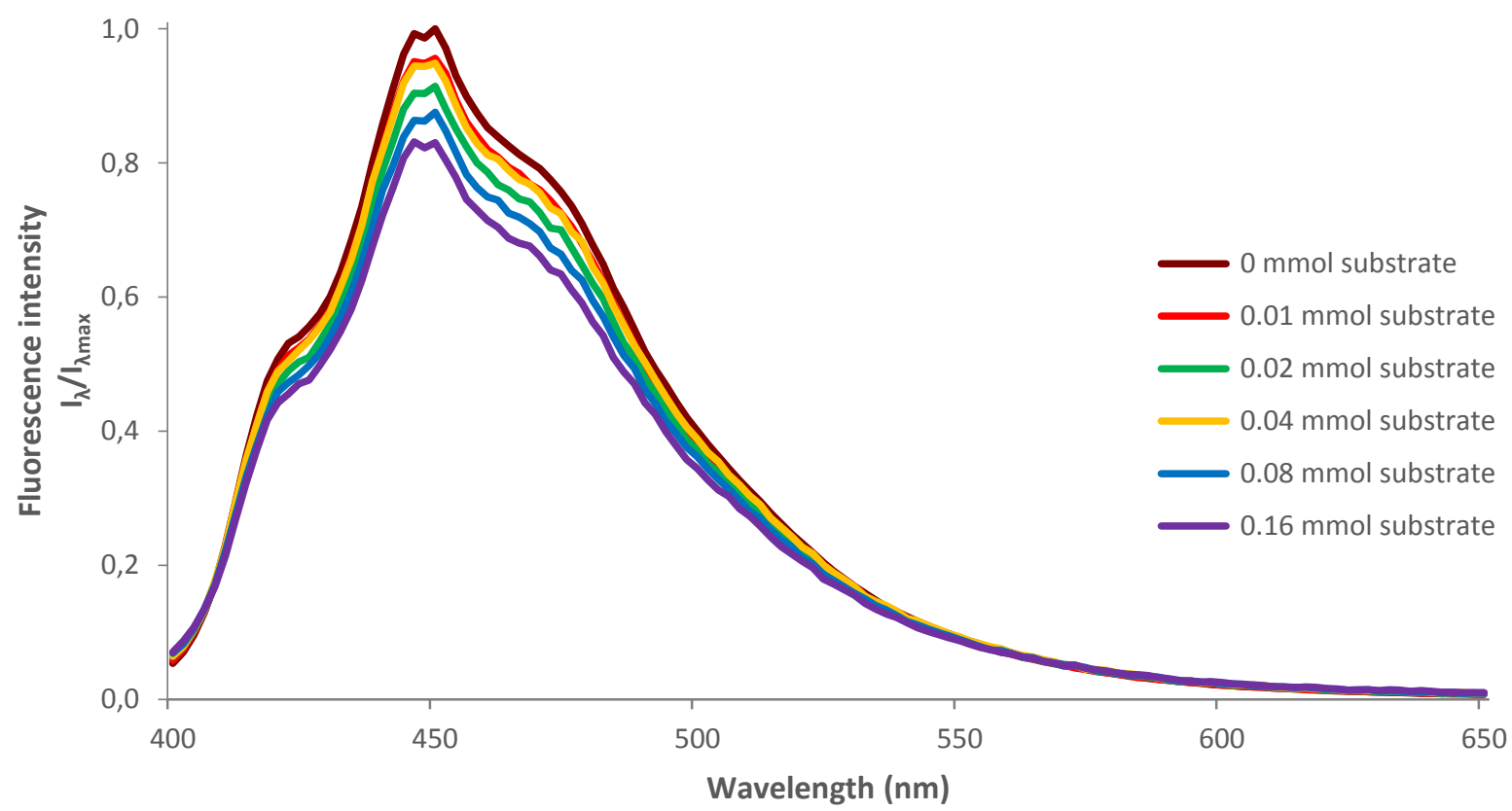

Figure S11. Emission spectra of PTH in DMSO $(0.17 \mathrm{mM})$ with varying concentrations of furan, normalized to PTH with no furan added.

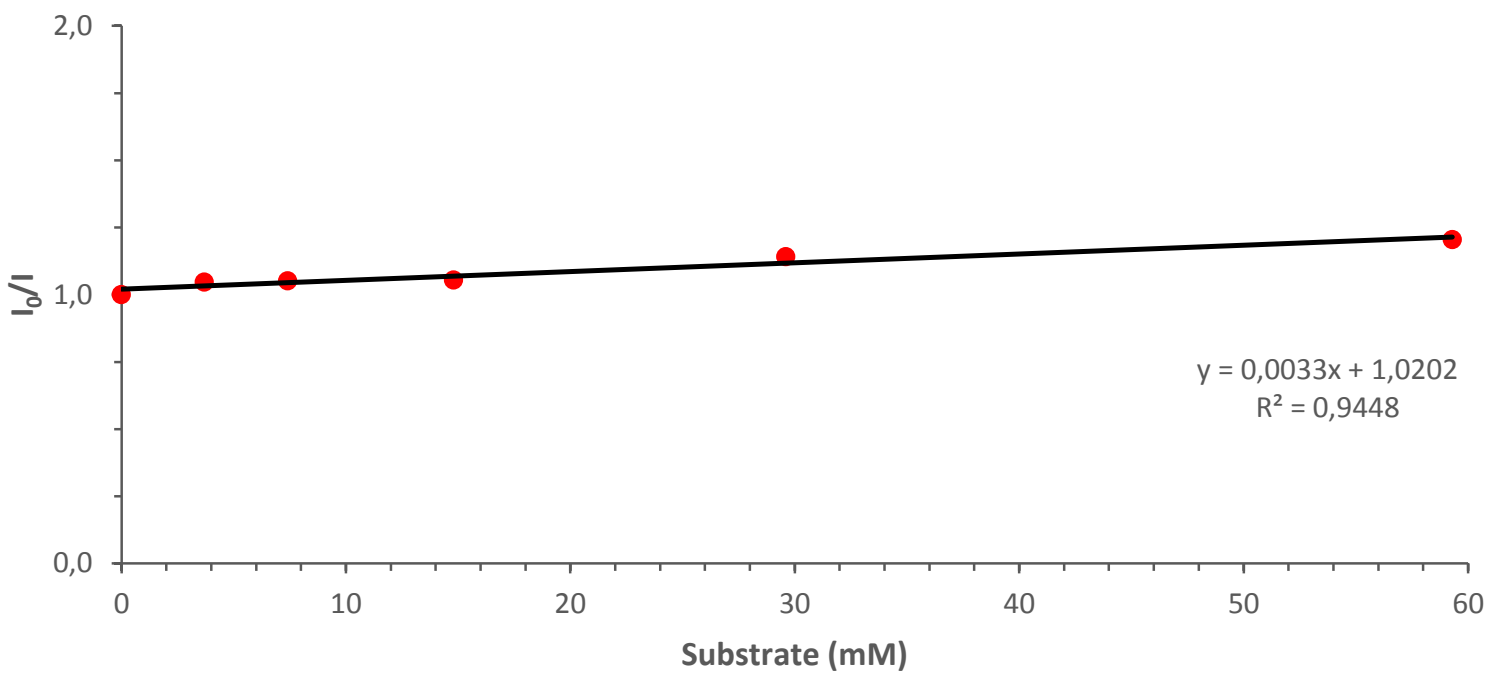

Figure S12. Stern-Volmer plot for PTH in DMSO $(0.17 \mathrm{mM})$ with varying furan.

$\mathrm{KSV}=3.3$ 


\section{SYNTHETIC PROCEDURES \& COMPOUND CHARACTERISATION}

\section{General Procedures}

\section{General Procedure A: Interrupted Pummerer Reactions of Arenes}

$\mathrm{Tf}_{2} \mathrm{O}$ (1.2 equiv.) was slowly added to a stirred solution of the corresponding arene $(0.2 \mathrm{mmol}, 1.0$ equiv.) and dibenzothiophene $S$-oxide (1.1 equiv.) in $\mathrm{CH}_{2} \mathrm{Cl}_{2}(0.1 \mathrm{M})$ at $-78{ }^{\circ} \mathrm{C}$ under a nitrogen atmosphere. The resulting solution was stirred at this temperature for 15 minutes before warming to room temperature. After stirring for $1 \mathrm{~h}, \mathrm{TLC}$ analysis showed complete consumption of the arene starting material, at which point the solvent was removed in vacuo. The sulfonium salt was then precipitated by the addition of $\mathrm{Et}_{2} \mathrm{O}$. The $\mathrm{Et}_{2} \mathrm{O}$ was then decanted off and the crude product was washed with further portions of $\mathrm{Et}_{2} \mathrm{O}$. The pure product was obtained by recrystallization $\left(\mathrm{CH}_{2} \mathrm{Cl}_{2} / \mathrm{Et}_{2} \mathrm{O}\right)$.

\section{General Procedure B: Photoredox-Catalyzed C-H/C-H Coupling of Arenes}

The dibenzothiophenium salt $(0.2 \mathrm{mmol}, 1.0$ equiv.) and 10-phenyl-phenothiazine (10 mol\%) were added to a reaction vial under a nitrogen atmosphere. DMSO $(0.2 \mathrm{M})$ was then added followed by the corresponding radical trap reagent. The reaction mixture was then irradiated with a Kessil $34 \mathrm{~W}$ blue LED lamp for $18 \mathrm{~h}$ before quenching with aqueous saturated $\mathrm{NaHCO}_{3}$ and dilution with EtOAc. The organic layer was washed with brine, dried using $\mathrm{MgSO}_{4}$, filtered, and concentrated in vacuo, to give the crude product, which was purified by column chromatography on silica gel.

\section{General Procedure C: Interrupted Pummerer-enabled, Photoredox-Catalyzed C- H/C-H Coupling of Arenes}

$\mathrm{Tf}_{2} \mathrm{O}$ (1.2 equiv.) was slowly added to a stirred solution of the arene $(0.2 \mathrm{mmol}, 1.0$ equiv.) and dibenzothiophene $\mathrm{S}$-oxide (1.1 equiv.) in $\mathrm{CH}_{2} \mathrm{Cl}_{2}(0.1 \mathrm{M})$ at $-78{ }^{\circ} \mathrm{C}$ under a nitrogen atmosphere. The resulting solution was stirred at this temperature for 15 minutes before warming to room temperature. After stirring for $1 \mathrm{~h}, \mathrm{TLC}$ analysis showed complete consumption of the arene starting material, at which point the solvent was removed in vacuo. 10-Phenyl-phenothiazine (10 mol\%) was then added to the reaction vial, followed by evacuation and refilling with nitrogen ( 3 cycles). DMSO $(0.2 \mathrm{M})$ was then added followed by the radical trap reagent. The reaction mixture was then irradiated with a Kessil $34 \mathrm{~W}$ blue LED lamp for $18 \mathrm{~h}$ before quenching with aqueous saturated $\mathrm{NaHCO}_{3}$ and dilution with EtOAc. The organic layer was washed with brine, dried using $\mathrm{MgSO}_{4}$, filtered, and concentrated in vacuo, to give the crude product, which was purified by column chromatography on silica gel. 


\section{General Procedure D: Interrupted Pummerer-enabled, Photoredox-Catalyzed C- $\mathrm{H} / \mathrm{C}-\mathrm{H}$ Coupling of Anilines \& Amide-Containing Substrates}

$\mathrm{Tf}_{2} \mathrm{O}$ (1.2 equiv.) was slowly added to a stirred solution of dibenzothiophene $\mathrm{S}$-oxide (1.1 equiv.) in $\mathrm{CH}_{2} \mathrm{Cl}_{2}(0.1 \mathrm{M})$ at $-78{ }^{\circ} \mathrm{C}$ under a nitrogen atmosphere. The resulting solution was stirred at this temperature for 1 hour before the addition of the aniline $(0.2 \mathrm{mmol}, 1.0$ equiv.) and a further 15 minutes of stirring. The solution was then warmed to room temperature. After stirring for $1 \mathrm{~h}, \mathrm{TLC}$ analysis showed complete consumption of the arene starting material, at which point the solvent was removed in vacuo. 10-Phenyl-phenothiazine (10 mol\%) was then added to the reaction vial, followed by evacuation and refilling with nitrogen ( 3 cycles). DMSO (0.2 M) was then added followed by the corresponding radical trap reagent. The reaction mixture was then irradiated with a Kessil $34 \mathrm{~W}$ blue LED lamp for $18 \mathrm{~h}$ before quenching with aqueous saturated $\mathrm{NaHCO}_{3}$ and dilution with EtOAc. The organic layer was washed with brine, dried using $\mathrm{MgSO}_{4}$, filtered, and concentrated in vacuo, to give the crude product, which was purified by column chromatography on silica gel. 


\section{Preparation of Starting Materials}

\section{Catalyst Preparation}

10-Phenyl-10H-phenothiazine, $\mathrm{S5}^{2}$<smiles>c1ccc(N2c3ccccc3Sc3ccccc32)cc1</smiles>

Phenothiazine (1.99 g, $10.0 \mathrm{mmol}, 1.00$ equiv.), sodium tert-butoxide $(2.88 \mathrm{~g}, 30.0 \mathrm{mmol}, 3.00$ equiv.), and $\mathrm{Pd}(\mathrm{dba})_{2}(0.29 \mathrm{~g}, 0.5 \mathrm{mmol}, 0.05$ equiv.) were added to a $50 \mathrm{~mL}$ round-bottom flask. The flask was fitted with a reflux condenser and sealed with a septum. The atmosphere was exchanged by applying vacuum and backfilling with nitrogen ( 3 cycles). lodobenzene $(1.1 \mathrm{~mL}, 10 \mathrm{mmol}, 1.00$ equiv.), tri-tert-butylphosphine (1.0 mL, $1 \mathrm{M}$ in toluene, $1.00 \mathrm{mmol}, 0.10$ equiv.), and toluene $(10 \mathrm{~mL})$ were added by syringe. The reaction mixture was then heated to $110{ }^{\circ} \mathrm{C}$ and stirred at this temperature for $16 \mathrm{~h}$. After cooling to room temperature, the reaction mixture was diluted with $\mathrm{CH}_{2} \mathrm{Cl}_{2}$ and washed with $\mathrm{H}_{2} \mathrm{O}$. The organic layer was dried over $\mathrm{MgSO}_{4}$, filtered and concentrated in vacuo. The crude product was purified by column chromatography on silica gel $\left(10 \% \mathrm{CHCl}_{3}\right.$ in hexane), to give $\mathbf{S 5}(2.46 \mathrm{~g}, 8.93 \mathrm{mmol}, 89 \%)$ as a pale yellow solid; $\delta_{H}\left(400 \mathrm{MHz}\right.$, DMSO- $\left.d_{6}\right) 6.16$ (dd, $J=8.0,1.3 \mathrm{~Hz}, 2 \mathrm{H}, \operatorname{Ar} H), 6.86(\mathrm{td}, J=7.4,1.3 \mathrm{~Hz}, 2 \mathrm{H}, \operatorname{Ar} H), 6.93(\mathrm{td}, J=8.0,1.7 \mathrm{~Hz}, 2 \mathrm{H}, \operatorname{Ar} H), 7.08$ (dd, $J=7.4,1.7 \mathrm{~Hz}, 2 \mathrm{H}, \operatorname{Ar} H$ ), $7.42(\mathrm{~d}, J=7.6 \mathrm{~Hz}, 2 \mathrm{H}, \operatorname{Ar} H), 7.54$ (d, J = 7.6 Hz, 1H, Ar H), 7.67 (t, $J=$ 7.6 Hz, 2H, Ar H); $\delta_{C}\left(100 \mathrm{MHz}, \mathrm{DMSO}-d_{6}\right) 116.1(\operatorname{ArCH}), 119.4(\operatorname{ArC}), 122.7(\mathrm{ArCH}), 126.7(\operatorname{ArCH})$, 127.3 ( $\mathrm{ArCH}), 128.4(\mathrm{ArCH}), 130.3(\mathrm{ArCH}), 131.0(\mathrm{ArCH}), 140.3(\operatorname{ArC}), 143.6(\operatorname{ArC})$.

The data is in accordance with the literature. ${ }^{2}$

\section{Substrate Preparation}

Dibenzo[b, $d]$ thiophene 5-oxide, $s 6^{5}$

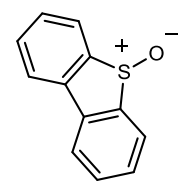

Dibenzothiophene $\left(9.2 \mathrm{~g}, 50.0 \mathrm{mmol}, 1.0\right.$ equiv.) was dissolved in $\mathrm{CH}_{2} \mathrm{Cl}_{2}(250 \mathrm{~mL})$ in a $500 \mathrm{ml}$ roundbottom flask and cooled to $0{ }^{\circ} \mathrm{C}$. $\mathrm{m}$-CPBA (11.2 g, 77\% in $\mathrm{H}_{2} \mathrm{O}, 50.0 \mathrm{mmol}, 1.0$ equiv.) was added portionwise over 30 minutes and the resulting suspension was stirred for a further 30 minutes. The 
reaction was then quenched with aqueous saturated $\mathrm{NaHCO}_{3}(150 \mathrm{~mL})$ and the organic layer was extracted using $\mathrm{CH}_{2} \mathrm{Cl}_{2}(3 \times 100 \mathrm{~mL})$. The combined organic layers were washed with aqueous saturated $\mathrm{NaHCO}_{3}(3 \times 150 \mathrm{~mL})$ and brine $(150 \mathrm{~mL})$, dried over $\mathrm{MgSO}_{4}$, filtered, and concentrated in vacuo. The crude product was purified by column chromatography on silica gel (10\% EtOAc in $\mathrm{CHCl}_{3}$ ), to give $\mathbf{S 6}(8.6 \mathrm{~g}, 42.9 \mathrm{mmol}, 86 \%)$ as a white solid; $\delta_{\mathrm{H}}\left(400 \mathrm{MHz}, \mathrm{CDCl}_{3}\right) 7.51(\mathrm{t}, J=7.6 \mathrm{~Hz}, 2 \mathrm{H}, \operatorname{Ar} H)$, $7.61(\mathrm{t}, J=7.6 \mathrm{~Hz}, 2 \mathrm{H}, \operatorname{Ar} H), 7.82(\mathrm{~d}, J=7.6 \mathrm{~Hz}, 2 \mathrm{H}, \operatorname{Ar} H), 8.00$ (d, J = 7.6 Hz, 2H, Ar H); $\delta_{\mathrm{C}}(100 \mathrm{MHz}$, $\left.\mathrm{CDCl}_{3}\right)$ 122.0 ( $\left.\mathrm{ArCH}\right), 127.7(\mathrm{ArCH}), 129.7(\mathrm{ArCH}), 132.7(\mathrm{ArCH}), 137.2(\operatorname{ArC}), 145.3(\operatorname{ArC})$.

The data is in accordance with the literature. ${ }^{5}$

5-(4-(tert-Butyl)phenyl)-5H-dibenzo[b, d] thiophen-5-ium trifluoromethanesulfonate, 2
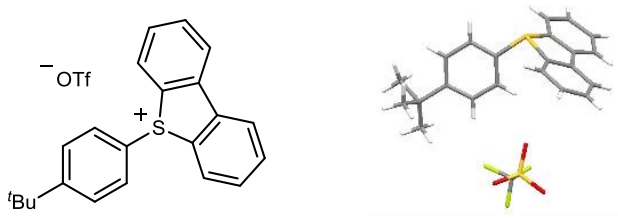

Prepared as described in General Procedure A: tert-butyl benzene $(0.78 \mathrm{~mL}, 5.00 \mathrm{mmol})$, dibenzothiophene $S$-oxide $(1.10 \mathrm{~g}, 5.50 \mathrm{mmol}), \mathrm{Tf}_{2} \mathrm{O}(1.00 \mathrm{~mL}, 6.00 \mathrm{mmol})$, after purification by recrystallization gave $2(2.04 \mathrm{~g}, 4.38 \mathrm{mmol}, 88 \%)$ as an off-white solid; m.p. (recrystallized from $\left.\mathrm{CH}_{2} \mathrm{Cl}_{2} / \mathrm{Et}_{2} \mathrm{O}\right) 184-186{ }^{\circ} \mathrm{C} ; \delta_{\mathrm{H}}\left(400 \mathrm{MHz}, \mathrm{CDCl}_{3}\right) 1.26\left(\mathrm{~s}, 9 \mathrm{H}, \mathrm{CH}_{3}\right), 7.47-7.55(\mathrm{~m}, 2 \mathrm{H}, \mathrm{Ar} \mathrm{H}), 7.56-$ $7.66(\mathrm{~m}, 4 \mathrm{H}, \operatorname{Ar} H), 7.84(\mathrm{td}, J=7.8,1.1 \mathrm{~Hz}, 2 \mathrm{H}, \operatorname{Ar} H), 8.15(\mathrm{~d}, J=8.2 \mathrm{~Hz}, 2 \mathrm{H}, \operatorname{Ar} H), 8.19(\mathrm{dd}, J=7.8$, $1.1 \mathrm{~Hz}, 2 \mathrm{H}, \mathrm{Ar} \mathrm{H}) ; \delta_{\mathrm{C}}\left(100 \mathrm{MHz}, \mathrm{CDCl}_{3}\right) 30.9\left(\mathrm{CH}_{3}\right), 35.6\left(\mathrm{CH}_{3} \mathrm{C}\right), 120.9\left(\mathrm{q}, J=320.6 \mathrm{~Hz}, \mathrm{CF}_{3}\right), 122.4(\mathrm{Ar}$ C), $124.2(\mathrm{ArCH}), 128.7(\mathrm{ArCH}), 129.0(\mathrm{ArCH}), 130.7(\mathrm{ArCH}), 131.8(\operatorname{ArCH}), 132.2(\operatorname{ArC}), 134.5(\operatorname{Ar}$ $\mathrm{CH}), 139.0(\operatorname{ArC}), 159.6(\operatorname{ArC}) ; \delta_{\mathrm{F}}\left(376 \mathrm{MHz}, \mathrm{CDCl}_{3}\right)-78.2 ; v_{\max }\left(\right.$ thin film/ $\left.\mathrm{cm}^{-1}\right) 736,761,783,837$, $1005,1027,1162,1222,1254,1448,2871,2907,2966,3062,3087 ; \mathrm{MS}_{\left(E^{+}\right)} \mathrm{m} / z 317.1\left(\mathrm{M}^{+}\right)$; HRMS $\mathrm{C}_{22} \mathrm{H}_{21}{ }^{+}\left(\mathrm{M}^{+}\right)$Expected 317.1358, Found 317.1352.

2 was further characterized by X-ray crystallographic analysis. CCDC: 1922367. 
5-Phenyl-5H-dibenzo[b,d]thiophen-5-ium trifluoromethanesulfonate, $\mathbf{S 2}$

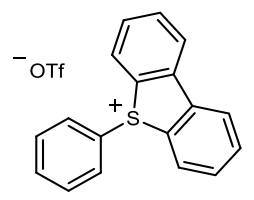

Prepared as described in General Procedure A: benzene $(0.09 \mathrm{~mL}, 1.00 \mathrm{mmol})$, dibenzothiophene $S$ oxide $(0.22 \mathrm{~g}, 1.10 \mathrm{mmol}), \mathrm{Tf}_{2} \mathrm{O}(0.20 \mathrm{~mL}, 1.20 \mathrm{mmol})$, after purification by recrystallization gave $\mathbf{S 2}$ ( $0.35 \mathrm{~g}, 0.84 \mathrm{mmol}, 84 \%$ ) as an off-white solid; m.p. (recrystallized from $\mathrm{CH}_{2} \mathrm{Cl}_{2} / \mathrm{Et}_{2} \mathrm{O}$ ) $177-179{ }^{\circ} \mathrm{C} ; \delta_{\mathrm{H}}$ $\left(400 \mathrm{MHz}, \mathrm{CDCl}_{3}\right) 7.48-7.56(\mathrm{~m}, 2 \mathrm{H}, \mathrm{Ar} H), 7.59-7.68(\mathrm{~m}, 5 \mathrm{H}, \mathrm{Ar} \mathrm{H}), 7.85$ (td, J = 7.8, 0.9 Hz, 2H, Ar $H), 8.14(\mathrm{~d}, J=8.2 \mathrm{~Hz}, 2 \mathrm{H}, \operatorname{Ar} H), 8.19(\mathrm{~d}, J=7.8 \mathrm{~Hz}, 2 \mathrm{H}, \operatorname{Ar} H) ; \delta_{\mathrm{C}}\left(100 \mathrm{MHz}, \mathrm{CDCl}_{3}\right) 120.8(\mathrm{q}, J=320.2$ $\left.\mathrm{Hz}, \mathrm{CF}_{3}\right), 124.2(\mathrm{ArCH}), 126.6(\operatorname{ArC}), 128.9(\mathrm{ArCH}), 130.8(\operatorname{ArCH}), 131.7(\operatorname{ArCH}), 131.9(\operatorname{ArCH}), 132.0$ $(\operatorname{ArC}), 134.6(\operatorname{ArCH}), 135.1(\operatorname{ArCH}), 139.1(\operatorname{ArC}) ; \delta_{F}\left(376 \mathrm{MHz}, \mathrm{CDCl}_{3}\right)-78.2 ; v_{\max }\left(\right.$ thin film/ $\mathrm{cm}^{-1}$ ) 734, 755, 997, 1026, 1049, 1222, 1251, 1448, 3011, 3062, 3088; MS (ES $\left.{ }^{+}\right) \mathrm{m} / \mathrm{z} 261.0\left(\mathrm{M}^{+}\right) ; \mathrm{HRMS} \mathrm{C}_{18} \mathrm{H}_{13} \mathrm{~S}^{+}$ $\left(\mathrm{M}^{+}\right)$Expected 261.0724, Found 261.0720.

5-(4-Methoxy-3-(methoxycarbonyl)phenyl)-5H-dibenzo[b, d]thiophen-5-ium trifluoromethanesulfonate, $\mathbf{S 3}$<smiles>COc1ccc(S(=O)(=O)c2ccccc2-c2ccccc2)c(OC)c1</smiles>

Prepared as described in General Procedure A: methyl 2-methoxybenzoate $(0.14 \mathrm{~mL}, 1.00 \mathrm{mmol})$, dibenzothiophene $S$-oxide $(0.22 \mathrm{~g}, 1.10 \mathrm{mmol}), \mathrm{Tf}_{2} \mathrm{O}(0.20 \mathrm{~mL}, 1.20 \mathrm{mmol})$, after purification by recrystallization gave $\mathbf{S 3}(0.46 \mathrm{~g}, 0.93 \mathrm{mmol}, 93 \%)$ as an off-white solid; m.p. (recrystallized from $\left.\mathrm{CH}_{2} \mathrm{Cl}_{2} / \mathrm{Et}_{2} \mathrm{O}\right) 162-164{ }^{\circ} \mathrm{C} ; \delta_{\mathrm{H}}\left(400 \mathrm{MHz}, \mathrm{CDCl}_{3}\right) 3.79\left(\mathrm{~s}, 3 \mathrm{H}, \mathrm{OCH}_{3}\right), 3.95\left(\mathrm{~s}, 3 \mathrm{H}, \mathrm{OCH}_{3}\right), 7.21(\mathrm{~d}, J=9.1$ $\mathrm{Hz}, 1 \mathrm{H}, \operatorname{Ar} H), 7.60(\mathrm{~d}, J=2.7 \mathrm{~Hz}, 1 \mathrm{H}, \operatorname{Ar} H), 7.62-7.68(\mathrm{~m}, 2 \mathrm{H}, \operatorname{Ar} H), 7.86(\mathrm{td}, J=7.9,1.1 \mathrm{~Hz}, 2 \mathrm{H}, \operatorname{Ar}$ $H$ ), $8.14(\mathrm{~d}, J=7.9 \mathrm{~Hz}, 2 \mathrm{H}, \operatorname{Ar} H), 8.18(\mathrm{~d}, J=7.9 \mathrm{~Hz}, 2 \mathrm{H}, \operatorname{Ar} H), 8.28(\mathrm{dd}, J=9.1,2.7 \mathrm{~Hz}, 1 \mathrm{H}, \operatorname{Ar} H) ; \delta_{\mathrm{C}}$ $\left(100 \mathrm{MHz}, \mathrm{CDCl}_{3}\right) 52.9\left(\mathrm{OCH}_{3}\right), 57.0\left(\mathrm{OCH}_{3}\right), 115.2(\mathrm{ArCH}), 120.9$ (q, J = 320.2 Hz, CF $), 123.7(\operatorname{ArC})$, 124.1 ( $\mathrm{ArCH}), 128.8(\operatorname{ArCH}), 132.0(\operatorname{ArCH}), 132.3(\operatorname{ArC}), 132.3(\operatorname{ArC}), 133.2(\operatorname{Ar~CH}), 134.6(\operatorname{ArCH})$, 138.5 ( $\mathrm{ArCH}), 138.8(\operatorname{ArC}), 164.0(\operatorname{ArC}), 164.4(C=0) ; \delta_{\mathrm{F}}\left(376 \mathrm{MHz}, \mathrm{CDCl}_{3}\right)-78.2 ; v_{\max }\left(\operatorname{thin}\right.$ film $/ \mathrm{cm}^{-1}$ ) $734,759,1028,1080,1155,1223,1250,1449,1488,1591,1728,2953,3013,3087$; MS (ES $\left.{ }^{+}\right) \mathrm{m} / \mathrm{z}$ $349.1\left(\mathrm{M}^{+}\right)$; $\mathrm{HRMS} \mathrm{C}_{21} \mathrm{H}_{17} \mathrm{O}_{3} \mathrm{~S}^{+}\left(\mathrm{M}^{+}\right)$Expected 349.0893, Found 349.0876. 
1-(4-(tert-Butyl)phenyl)tetrahydro-1H-thiophen-1-ium trifluoromethanesulfonate, $\mathbf{S 4}$<smiles>Brc1ccc([SH]2(=[131I])CCCC2)cc1</smiles>

Prepared as described in General Procedure A: tert-butyl benzene $(0.78 \mathrm{~mL}, 5.00 \mathrm{mmol})$, tetrahydrothiophene-S-oxide $(0.50 \mathrm{~mL}, 5.50 \mathrm{mmol}), \mathrm{Tf}_{2} \mathrm{O}(1.00 \mathrm{~mL}, 6.00 \mathrm{mmol})$, after purification by recrystallization $\left(\mathrm{CH}_{2} \mathrm{Cl}_{2} / \mathrm{Et}_{2} \mathrm{O}\right)$, gave $\mathbf{S} 4(1.46 \mathrm{~g}, 3.95 \mathrm{mmol}, 79 \%)$ as a grey solid; m.p. (recrystallized from $\left.\mathrm{CH}_{2} \mathrm{Cl}_{2} / \mathrm{Et}_{2} \mathrm{O}\right) 106-108{ }^{\circ} \mathrm{C} ; \delta_{\mathrm{H}}\left(400 \mathrm{MHz}, \mathrm{CDCl}_{3}\right) 1.33\left(\mathrm{~s}, 9 \mathrm{H}, \mathrm{CH}_{3}\right), 2.49-2.63\left(\mathrm{~m}, 4 \mathrm{H}, \mathrm{CH}_{2}\right), 3.57$ $-3.70\left(\mathrm{~m}, 2 \mathrm{H}, \mathrm{CH}_{2}\right), 4.16-4.29\left(\mathrm{~m}, 2 \mathrm{H}, \mathrm{CH}_{2}\right), 7.60-7.69(\mathrm{~m}, 2 \mathrm{H}, \operatorname{Ar} H), 7.66-7.74(\mathrm{~m}, 2 \mathrm{H}, \operatorname{Ar} H) ; \delta_{\mathrm{c}}$ $\left(100 \mathrm{MHz}, \mathrm{CDCl}_{3}\right) 29.3\left(\mathrm{CH}_{2}\right), 31.0\left(\mathrm{CH}_{3}\right), 35.5\left(\mathrm{CH}_{3} \mathrm{C}\right), 48.8\left(\mathrm{CH}_{2}\right), 120.8\left(\mathrm{q}, \mathrm{J}=320.4 \mathrm{~Hz}, \mathrm{CF}_{3}\right), 122.5(\mathrm{Ar}$ C), $128.7(\mathrm{ArCH}), 129.7(\mathrm{ArCH}), 158.7(\mathrm{ArC}) ; \delta_{\mathrm{F}}\left(376 \mathrm{MHz}, \mathrm{CDCl}_{3}\right)-78.3 ; v_{\max }\left(\right.$ thin film $\left./ \mathrm{cm}^{-1}\right)$ 735, 843, 871, 1030, 1158, 1250, 1266, 2873, 2908, 2961, 3025; MS $\left(\mathrm{ES}^{+}\right) \mathrm{m} / \mathrm{z} 221.1\left(\mathrm{M}^{+}\right) ; \mathrm{HRMS} \mathrm{C}_{14} \mathrm{H}_{21}{ }^{+}\left(\mathrm{M}^{+}\right)$ Expected 221.1358, Found 221.1355.

Phenyl pivalate, $S \mathbf{7}^{6}$<smiles>O=C([Se])Oc1ccccc1</smiles>

Triethylamine ( $2.20 \mathrm{~mL}, 15.9 \mathrm{mmol}, 2.0$ equiv.) and pivaloyl chloride ( $1.10 \mathrm{~mL}, 8.77 \mathrm{mmol}, 1.1$ equiv.) were added sequentially to a stirred solution of phenol $(0.75 \mathrm{~g}, 7.97 \mathrm{mmol}, 1.0$ equiv.) in THF (10 mL) at $0{ }^{\circ} \mathrm{C}$. After 15 minutes, the reaction mixture was warmed to ambient temperature and was stirred for a further 18 hours, before removal of the solvent in vacuo. The crude product was purified by column chromatography on silica gel (1\% EtOAc in hexanes), to give $\mathbf{S 7}(1.21 \mathrm{~g}, 6.77 \mathrm{mmol}, 85 \%)$ as a colourless oil; $\delta_{\mathrm{H}}\left(400 \mathrm{MHz}, \mathrm{CDCl}_{3}\right) 1.37\left(\mathrm{~s}, 9 \mathrm{H}, \mathrm{CH}_{3}\right), 7.03-7.09(\mathrm{~m}, 2 \mathrm{H}, \mathrm{Ar} H), 7.18-7.25(\mathrm{~m}, 1 \mathrm{H}, \mathrm{Ar}$ H), 7.34 - $7.42(\mathrm{~m}, 2 \mathrm{H}, \mathrm{Ar} \mathrm{H}) ; \delta_{\mathrm{C}}\left(100 \mathrm{MHz}, \mathrm{CDCl}_{3}\right) 27.3\left(\mathrm{CH}_{3}\right), 39.2\left(\mathrm{CH}_{3} \mathrm{C}\right), 121.6(\mathrm{ArCH}), 125.7(\mathrm{Ar}$ $\mathrm{CH}), 129.5(\mathrm{ArCH}), 151.3(\operatorname{Ar} C), 177.2(\mathrm{C}=\mathrm{O})$.

The data is in accordance with the literature. ${ }^{7}$ 
$N, N$-Diethyl-2-methoxybenzamide, $\mathbf{S} 8^{8}$<smiles>COc1ccccc1C(=O)Nc1ccccc1</smiles>

Dimethyl sulfate $(0.32 \mathrm{~mL}, 3.3 \mathrm{mmol}, 1.1$ equiv.) was added to a stirred solution of $\mathrm{N}, \mathrm{N}$-diethyl-2hydroxybenzamide ( $0.58 \mathrm{~g}, 3.0 \mathrm{mmol}, 1.0$ equiv.) and $\mathrm{K}_{2} \mathrm{CO}_{3}(0.46 \mathrm{~g}, 3.3 \mathrm{mmol}, 1.1$ equiv.) in acetone $(15 \mathrm{~mL})$ at ambient temperature. The reaction mixture was stirred for 18 hours, before removal of the solvent in vacuo. The crude residue was then diluted with $\mathrm{CH}_{2} \mathrm{Cl}_{2}(25 \mathrm{~mL})$, washed with water (2 $\times 25 \mathrm{~mL}$ ) and brine $(25 \mathrm{~mL})$, dried over $\mathrm{MgSO}_{4}$, filtered, and concentrated in vacuo. The crude product was purified by column chromatography on silica gel (40\% EtOAc in hexanes), to give $\mathbf{5 8}$ (0.61 g, $2.95 \mathrm{mmol}, 98 \%)$ as a white solid; $\delta_{\mathrm{H}}\left(400 \mathrm{MHz}, \mathrm{CDCl}_{3}\right) 1.02\left(\mathrm{t}, J=7.1 \mathrm{~Hz}, 3 \mathrm{H}, \mathrm{CH}_{3}\right), 1.24(\mathrm{t}, J=$ $\left.7.1 \mathrm{~Hz}, 3 \mathrm{H}, \mathrm{CH}_{3}\right), 3.14\left(\mathrm{q}, J=7.1 \mathrm{~Hz}, 2 \mathrm{H}, \mathrm{CH}_{2}\right), 3.46-3.69\left(\mathrm{~m}, 2 \mathrm{H}, \mathrm{CH}_{2}\right), 3.81\left(\mathrm{~s}, 3 \mathrm{H}, \mathrm{OCH}_{3}\right), 6.89(\mathrm{dd}, J=$ 8.4, $0.9 \mathrm{~Hz}, 1 \mathrm{H}, \operatorname{Ar} H), 6.96(\mathrm{td}, J=7.4,0.9 \mathrm{~Hz}, 1 \mathrm{H}, \operatorname{Ar} H), 7.18(\mathrm{dd}, J=7.4,1.7 \mathrm{~Hz}, 1 \mathrm{H}, \operatorname{Ar} H), 7.31$ (ddd, $J=8.4,7.4,1.7 \mathrm{~Hz}, 1 \mathrm{H}, \mathrm{Ar} H) ; \delta_{\mathrm{C}}(100 \mathrm{MHz}, \mathrm{CDCl}) 13.0\left(\mathrm{CH}_{3}\right), 14.1\left(\mathrm{CH}_{3}\right), 38.9\left(\mathrm{CH}_{2}\right), 42.9\left(\mathrm{CH}_{2}\right), 55.6$ $\left(\mathrm{OCH}_{3}\right), 111.1(\mathrm{ArCH}), 120.9(\mathrm{ArCH}), 127.2(\operatorname{ArC}), 127.6(\mathrm{ArCH}), 130.0(\operatorname{ArCH}), 155.3(\operatorname{ArC}), 168.9(\operatorname{Ar}$ $C=0$ ).

The data is in accordance with the literature. ${ }^{9}$

(2R,3R,4S,5R,6S)-2-(Acetoxymethyl)-6-(2-(acetoxymethyl)phenoxy)tetrahydro-2H-pyran-3,4,5-triyl triacetate, $\mathbf{s} \mathbf{g}^{10}$<smiles>O=C(O)COc1ccccc1OC1O[C@H](CO)C(O)[C@H](O)[C@H]1O</smiles>

Acetic anhydride ( $9.50 \mathrm{~mL}, 100 \mathrm{mmol}, 10$ equiv.) was added to a stirred solution of salicin ( $2.86 \mathrm{~g}, 10$ mmol, 1.0 equiv.) and $\mathrm{K}_{2} \mathrm{CO}_{3}(2.76 \mathrm{~g}, 20 \mathrm{mmol}, 2.0$ equiv.) in dioxane (40 mL) at ambient temperature. The resulting suspension was then heated to $60{ }^{\circ} \mathrm{C}$ for 16 hours before removing the solvent in vacuo. The crude residue was then diluted with EtOAc $(50 \mathrm{~mL})$, washed with water $(2 \times 50$ $\mathrm{mL}$ ) and brine $(50 \mathrm{~mL})$, dried over $\mathrm{MgSO}_{4}$, filtered, and concentrated in vacuo. The crude product was purified by recrystallization (1:1 EtOAc/hexanes), to give $\mathbf{S 9}(4.62 \mathrm{~g}, 9.3 \mathrm{mmol}, 93 \%)$ as a white solid; $\delta_{\mathrm{H}}\left(400 \mathrm{MHz}, \mathrm{CDCl}_{3}\right) 2.03\left(\mathrm{~s}, 3 \mathrm{H}, \mathrm{CH}_{3}\right), 2.04\left(\mathrm{~s}, 3 \mathrm{H}, \mathrm{CH}_{3}\right), 2.06\left(\mathrm{~s}, 3 \mathrm{H}, \mathrm{CH}_{3}\right), 2.09\left(\mathrm{~s}, 6 \mathrm{H}, \mathrm{CH}_{3}\right), 3.85$ (ddd, $J=10.0,5.3,2.5 \mathrm{~Hz}, 1 \mathrm{H}$, Alk CH), 4.18 (dd, $J=12.3,2.5 \mathrm{~Hz}, 1 \mathrm{H}$, Alk $\mathrm{CH}_{2}$ ), 4.27 (dd, $J=12.3,5.3$ $\mathrm{Hz}, 1 \mathrm{H}$, Alk $\left.\mathrm{CH}_{2}\right), 5.01-5.22\left(\mathrm{~m}, 4 \mathrm{H}\right.$, Alk CH \& Bn CH $\left.\mathrm{CH}_{2}\right), 5.25-5.35(\mathrm{~m}, 2 \mathrm{H}$, Alk CH$), 7.08(\mathrm{t}, J=7.7 \mathrm{~Hz}$, 
2H, Ar H), $7.24-7.30(\mathrm{~m}, 1 \mathrm{H}, \operatorname{Ar} H), 7.34(\mathrm{dd}, J=7.7,1.5 \mathrm{~Hz}, 1 \mathrm{H}, \operatorname{Ar} H) ; \delta_{\mathrm{c}}\left(100 \mathrm{MHz}, \mathrm{CDCl}_{3}\right) 20.7$ $\left(\mathrm{CH}_{3}\right), 20.7\left(\mathrm{CH}_{3}\right), 20.7\left(\mathrm{CH}_{3}\right), 20.8\left(\mathrm{CH}_{3}\right), 21.1\left(\mathrm{CH}_{3}\right), 61.2\left(\mathrm{BnCH}_{2}\right), 62.0\left(\right.$ Alk $\left.\mathrm{CH}_{2}\right), 68.4$ (Alk $\left.\mathrm{CH}\right), 71.1$ (Alk $C H$ ), 72.1 (Alk CH), 72.8 (Alk CH), 99.5 (Alk CH), 116.0 (Ar CH), 123.7 ( $\operatorname{ArCH}), 126.4(\operatorname{ArC}), 129.5$ $(\operatorname{ArCH}), 129.7(\operatorname{ArCH}), 154.7(\operatorname{ArC}), 169.4(C=0), 169.5(C=0), 170.3(C=0), 170.7(C=0), 170.8(C=0)$. The data is in accordance with the literature. ${ }^{10}$

N-(1-(2,6-Dimethylphenoxy)propan-2-yl)acetamide, $\mathbf{S 1 0}{ }^{11}$<smiles>CC(=O)NC(C)COc1c(C)cccc1C</smiles>

Acetyl chloride $(0.50 \mathrm{~mL}, 6.95 \mathrm{mmol}, 1.5$ equiv.) was slowly added to a stirred suspension of mexiletine hydrochloride $\left(1.00 \mathrm{~g}, 4.64 \mathrm{mmol}, 1.0\right.$ equiv.) in EtOAc $(5 \mathrm{~mL})$ and $5 \mathrm{~N} \mathrm{NaOH}(5 \mathrm{~mL})$ at $0{ }^{\circ} \mathrm{C}$. After the addition, the reaction mixture was warmed to ambient temperature and stirred for 1 hour. The mixture was then added to water $(10 \mathrm{~mL})$ and the organic layer was extracted using EtOAc $(3 \times$ $15 \mathrm{~mL})$. The combined organic layers were washed with aqueous saturated $\mathrm{NaHCO}_{3}(3 \times 15 \mathrm{~mL})$ and brine (15 mL), dried over $\mathrm{MgSO}_{4}$, filtered, and concentrated in vacuo, to give $\mathbf{S 1 0}(0.99 \mathrm{~g}, 4.50 \mathrm{mmol}$, 97\%) as a white solid; $\delta_{\mathrm{H}}\left(400 \mathrm{MHz}, \mathrm{CDCl}_{3}\right) 1.41\left(\mathrm{~d}, J=6.9 \mathrm{~Hz}, 3 \mathrm{H}, \mathrm{CH}_{3}\right), 2.03\left(\mathrm{~s}, 3 \mathrm{H}, \mathrm{CH}_{3}\right), 2.26(\mathrm{~s}, 6 \mathrm{H}$, $\left.\mathrm{CH}_{3}\right), 3.71\left(\mathrm{dd}, J=9.1,3.5 \mathrm{~Hz}, 1 \mathrm{H}, \mathrm{CH}_{2}\right), 3.80\left(\mathrm{dd}, J=9.1,3.5 \mathrm{~Hz}, 1 \mathrm{H}, \mathrm{CH}_{2}\right), 4.24-4.44(\mathrm{~m}, 1 \mathrm{H}$, Alk $\mathrm{CH})$, $5.91(\mathrm{br} \mathrm{s}, 1 \mathrm{H}, \mathrm{NH}), 6.93(\mathrm{dd}, J=8.4,6.5 \mathrm{~Hz}, 1 \mathrm{H}, \operatorname{Ar} H), 6.99-7.03(\mathrm{~m}, 2 \mathrm{H}, \operatorname{Ar} H) ; \delta_{\mathrm{c}}\left(100 \mathrm{MHz}, \mathrm{CDCl}_{3}\right)$ $16.3\left(\mathrm{CH}_{3}\right), 17.9\left(\mathrm{CH}_{3}\right), 23.6\left(\mathrm{CH}_{3}\right), 45.6(\mathrm{Alk} \mathrm{CH}), 74.0\left(\mathrm{CH}_{2}\right), 124.3(\mathrm{ArCH}), 129.2(\mathrm{ArCH}), 130.8(\operatorname{ArC})$, $155.0(\operatorname{ArC}), 169.5(C=0)$.

The data is in accordance with the literature. ${ }^{11}$ 
Interrupted Pummerer-Enabled, Photoredox-Catalyzed $\mathrm{C}-\mathrm{H} / \mathrm{C}-\mathrm{H}$ Coupling of Arenes

\section{Substrate Scope}

2-(4-(tert-Butyl)phenyl)furan, $\mathbf{S 1}^{12}$<smiles>Brc1ccc(-c2ccco2)cc1</smiles>

Prepared as described in General Procedure C: tert-butyl benzene (31 $\mu \mathrm{L}, 0.20 \mathrm{mmol}$ ), dibenzothiophene $S$-oxide ( $44 \mathrm{mg}, 0.22 \mathrm{mmol}$ ), $\mathrm{Tf}_{2} \mathrm{O}$ ( $41 \mu \mathrm{L}, 0.24 \mathrm{mmol}$ ), PTH (5.5 mg, $0.02 \mathrm{mmol}$ ), furan $(0.29 \mathrm{~mL}, 4.00 \mathrm{mmol}, 20$ equiv.), after purification by column chromatography on silica gel (20\% $\mathrm{CHCl}_{3}$ in hexanes), gave $\mathbf{S} 1(23.2 \mathrm{mg}, 0.12 \mathrm{mmol}, 58 \%)$ as a white solid; $\delta_{\mathrm{H}}\left(500 \mathrm{MHz}, \mathrm{CDCl}_{3}\right)$ $1.34\left(\mathrm{~s}, 9 \mathrm{H}, \mathrm{CH}_{3}\right), 6.46(\mathrm{dd}, J=3.3,1.8 \mathrm{~Hz}, 1 \mathrm{H}, \operatorname{Ar} H), 6.60(\mathrm{dd}, J=3.3,0.8 \mathrm{~Hz}, 1 \mathrm{H}, \operatorname{Ar} H), 7.41(\mathrm{~d}, J=8.5$ $\mathrm{Hz}, 2 \mathrm{H}, \operatorname{Ar} H), 7.45(\mathrm{dd}, J=1.8,0.8 \mathrm{~Hz}, 1 \mathrm{H}, \operatorname{Ar} H), 7.61(\mathrm{~d}, J=8.5 \mathrm{~Hz}, 2 \mathrm{H}, \operatorname{Ar} H) ; \delta_{\mathrm{C}}\left(125 \mathrm{MHz}, \mathrm{CDCl}_{3}\right)$ 31.4 $\left(\mathrm{CH}_{3}\right), 34.8\left(\mathrm{CH}_{3} \mathrm{C}\right), 104.5(\mathrm{ArCH}), 111.7(\mathrm{ArCH}), 123.7(\operatorname{ArCH}), 125.7(\operatorname{ArCH}), 128.4(\operatorname{ArC}), 141.9$ $(\operatorname{ArCH}), 150.5(\operatorname{ArC}), 154.3(\operatorname{ArC})$.

The data is in accordance with the literature. ${ }^{12}$

1-Methyl-2-phenyl-1H-pyrrole, $7^{13}$<smiles></smiles>

Prepared as described in General Procedure C: benzene $(18 \mu \mathrm{L}, 0.20 \mathrm{mmol})$, dibenzothiophene $\mathrm{S}$ oxide (44 mg, $0.22 \mathrm{mmol}), \mathrm{Tf}_{2} \mathrm{O}(41 \mu \mathrm{L}, 0.24 \mathrm{mmol}), \mathrm{PTH}(5.5 \mathrm{mg}, 0.02 \mathrm{mmol}), \mathrm{N}$-methylpyrrole (1.0 $\mathrm{mL}, 11.3 \mathrm{mmol}, 56$ equiv.), after purification by column chromatography on silica gel $\left(20 \% \mathrm{CHCl}_{3}\right.$ in hexanes), gave $7(15.4 \mathrm{mg}, 0.10 \mathrm{mmol}, 49 \%)$ as an off-white solid; $\delta_{\mathrm{H}}\left(400 \mathrm{MHz}, \mathrm{CDCl}_{3}\right) 3.68(\mathrm{~s}, 3 \mathrm{H}$, $\left.\mathrm{CH}_{3}\right), 6.22(\mathrm{dd}, J=3.6,2.6 \mathrm{~Hz}, 1 \mathrm{H}, \operatorname{Ar} H), 6.24(\mathrm{dd}, J=3.6,1.9 \mathrm{~Hz}, 1 \mathrm{H}, \operatorname{Ar} H), 6.73(\mathrm{dd}, J=2.6,1.9 \mathrm{~Hz}$, 1H, $\operatorname{Ar} H), 7.28-7.35(\mathrm{~m}, 1 \mathrm{H}, \operatorname{Ar} H), 7.37-7.44(\mathrm{~m}, 4 \mathrm{H}, \operatorname{Ar} H) ; \delta_{\mathrm{C}}\left(100 \mathrm{MHz}, \mathrm{CDCl}_{3}\right) 35.2\left(\mathrm{CH}_{3}\right), 107.9$ ( $\operatorname{ArCH}), 108.8(\mathrm{ArCH}), 123.8(\operatorname{ArCH}), 126.9(\mathrm{ArCH}), 128.5(\operatorname{ArCH}), 128.8(\operatorname{ArCH}), 133.5(\operatorname{ArC}), 134.7$ $(\operatorname{Ar} C)$.

The data is in accordance with the literature. ${ }^{13}$ 
2-(4-Ethylphenyl)-1-methyl-1H-pyrrole, 8<smiles></smiles>

Prepared as described in General Procedure C: ethylbenzene ( $25 \mu \mathrm{L}, 0.20 \mathrm{mmol}$ ), dibenzothiophene S-oxide (44 mg, $0.22 \mathrm{mmol}$ ), Tf $\mathrm{O}_{2} \mathrm{O}(41 \mu \mathrm{L}, 0.24 \mathrm{mmol}$ ), PTH (5.5 mg, $0.02 \mathrm{mmol}$ ), $\mathrm{N}$-methylpyrrole $(0.36 \mathrm{~mL}, 4.00 \mathrm{mmol}, 20$ equiv.), after purification by column chromatography on silica gel ( $20 \%$ $\mathrm{CHCl}_{3}$ in hexanes), gave $8(19.2 \mathrm{mg}, 0.10 \mathrm{mmol}, 52 \%)$ as a colourless oil; $\delta_{\mathrm{H}}\left(400 \mathrm{MHz}, \mathrm{CDCl}_{3}\right) 1.28$ (td, $\left.J=7.6,0.8 \mathrm{~Hz}, 3 \mathrm{H}, \mathrm{CH}_{3}\right), 2.69\left(\mathrm{q}, \mathrm{J}=7.6 \mathrm{~Hz}, 2 \mathrm{H}, \mathrm{CH}_{2}\right), 3.67\left(\mathrm{~s}, 3 \mathrm{H}, \mathrm{NCH}_{3}\right), 6.18-6.23(\mathrm{~m}, 2 \mathrm{H}, \operatorname{Ar~H})$, $6.68-6.73(\mathrm{~m}, 1 \mathrm{H}, \operatorname{Ar} H), 7.24(\mathrm{~d}, \mathrm{~J}=8.1 \mathrm{~Hz}, 2 \mathrm{H}, \operatorname{Ar} H), 7.33(\mathrm{~d}, \mathrm{~J}=8.1 \mathrm{~Hz}, 2 \mathrm{H}, \operatorname{Ar} H) ; \delta_{\mathrm{c}}(100 \mathrm{MHz}$, $\left.\mathrm{CDCl}_{3}\right)$ 15.6 $\left(\mathrm{CH}_{3}\right), 28.7\left(\mathrm{CH}_{2}\right), 35.1\left(\mathrm{NCH}_{3}\right), 107.8(\mathrm{ArCH}), 108.5(\mathrm{Ar} \mathrm{CH}), 123.4(\mathrm{ArCH}), 128.0(\mathrm{ArCH})$, 128.8 (ArCH), $130.8(\operatorname{ArC}), 134.8(\operatorname{Ar} C), 143.0(\operatorname{Ar} C) ; v_{\max }\left(\right.$ thin film $\left./ \mathrm{cm}^{-1}\right)$ 834, 909, 982, 1057, 1089, $1242,1308,1414,1475,2871,2929,2962 ; \mathrm{MS}$ (GCMS) $\mathrm{m} / \mathrm{z} 185.1$ (M); HRMS $\mathrm{C}_{13} \mathrm{H}_{16} \mathrm{~N}^{+}\left(\mathrm{M}+\mathrm{H}^{+}\right)$ Expected 186.1277, Found 186.1276.

2-(4-Isopropylphenyl)-1-methyl-1H-pyrrole, 9<smiles></smiles>

Prepared as described in General Procedure C: cumene $(28 \mu \mathrm{L}, 0.20 \mathrm{mmol})$, dibenzothiophene $\mathrm{S}$ oxide (44 mg, $0.22 \mathrm{mmol}$ ), Tf $\mathrm{T}_{2} \mathrm{O}(41 \mu \mathrm{L}, 0.24 \mathrm{mmol}$ ), PTH ( $5.5 \mathrm{mg}, 0.02 \mathrm{mmol}), N$-methylpyrrole (0.36 $\mathrm{mL}, 4.00 \mathrm{mmol}, 20$ equiv.), after purification by column chromatography on silica gel $\left(20 \% \mathrm{CHCl}_{3}\right.$ in hexanes), gave 9 (19.4 mg, $0.10 \mathrm{mmol}, 49 \%)$ as a colourless oil; $\delta_{\mathrm{H}}\left(400 \mathrm{MHz}, \mathrm{CDCl}_{3}\right) 1.30$ (d, J = 6.9 $\left.\mathrm{Hz}, 6 \mathrm{H}, \mathrm{CH}_{3}\right), 2.95$ (hept, $J=6.9 \mathrm{~Hz}, 1 \mathrm{H}, \mathrm{Alk} \mathrm{CH}$ ), $3.67\left(\mathrm{~s}, 3 \mathrm{H}, \mathrm{NCH}_{3}\right), 6.16-6.26(\mathrm{~m}, 2 \mathrm{H}, \mathrm{Ar} \mathrm{H}), 6.67-$ $6.77(\mathrm{~m}, 1 \mathrm{H}, \operatorname{Ar} H), 7.27(\mathrm{~d}, J=8.2 \mathrm{~Hz}, 2 \mathrm{H}, \operatorname{Ar} H), 7.34(\mathrm{~d}, J=8.2 \mathrm{~Hz}, 2 \mathrm{H}, \operatorname{Ar} H) ; \delta_{\mathrm{C}}\left(100 \mathrm{MHz}, \mathrm{CDCl}_{3}\right)$ $24.1\left(\mathrm{CH}_{3}\right), 34.0$ (Alk CH), $35.2\left(\mathrm{NCH}_{3}\right), 107.8(\mathrm{ArCH}), 108.5(\mathrm{ArCH}), 123.4(\mathrm{ArCH}), 126.5(\mathrm{Ar} \mathrm{CH})$, 128.8 (ArCH), $131.0(\operatorname{ArC}), 134.8(\operatorname{ArC}), 147.5(\operatorname{ArC}) ; v_{\max }\left(\operatorname{thin} \mathrm{film} / \mathrm{cm}^{-1}\right)$ 835, 909, 982, 1058, 1241, 1309, 1361, 1458, 1475, 2867, 2926, 2957; MS (GCMS) m/z 199.1 (M); HRMS C ${ }_{14} \mathrm{H}_{18} \mathrm{~N}^{+}\left(\mathrm{M}^{+} \mathrm{H}^{+}\right)$ Expected 200.1434, Found 200.1432. 
2-(4-(tert-Butyl)phenyl)-1-methyl-1H-pyrrole, $\mathbf{3}^{14}$<smiles>c1ccc(-c2cccn2-c2ccccc2)cc1</smiles>

Prepared as described in General Procedure C: tert-butyl benzene (31 $\mu \mathrm{L}, 0.20 \mathrm{mmol}$ ), dibenzothiophene $S$-oxide (44 mg, $0.22 \mathrm{mmol}$ ), $\mathrm{Tf}_{2} \mathrm{O}$ ( $41 \mu \mathrm{L}, 0.24 \mathrm{mmol}$ ), PTH (5.5 mg, $\left.0.02 \mathrm{mmol}\right), \mathrm{N}$ methylpyrrole $(0.36 \mathrm{~mL}, 4.00 \mathrm{mmol}, 20$ equiv.), after purification by column chromatography on silica gel (20\% $\mathrm{CHCl}_{3}$ in hexanes), gave $3(21.8 \mathrm{mg}, 0.10 \mathrm{mmol}, 51 \%)$ as a colourless oil; Reaction with excess $N$-methylpyrrole $\left(1.0 \mathrm{~mL}, 11.3 \mathrm{mmol}, 56\right.$ equiv.), gave $3(28.5 \mathrm{mg}, 0.13 \mathrm{mmol}, 67 \%) ; \delta_{H}(400$ $\left.\mathrm{MHz}, \mathrm{CDCl}_{3}\right) 1.37\left(\mathrm{~s}, 9 \mathrm{H}, \mathrm{CH}_{3}\right), 3.68\left(\mathrm{~s}, 3 \mathrm{H}, \mathrm{NCH}_{3}\right), 6.19-6.25(\mathrm{~m}, 2 \mathrm{H}, \mathrm{Ar} H), 6.69-6.75(\mathrm{~m}, 1 \mathrm{H}, \mathrm{Ar} H)$, $7.36(\mathrm{~d}, J=8.5 \mathrm{~Hz}, 2 \mathrm{H}, \operatorname{Ar} H), 7.44(\mathrm{~d}, J=8.5 \mathrm{~Hz}, 2 \mathrm{H}, \operatorname{Ar} H) ; \delta_{\mathrm{C}}\left(100 \mathrm{MHz}, \mathrm{CDCl}_{3}\right) 31.5\left(\mathrm{CH}_{3}\right), 34.7$ $\left(\mathrm{CH}_{3} \mathrm{C}\right), 35.2\left(\mathrm{NCH}_{3}\right), 107.8(\mathrm{Ar} \mathrm{CH}), 108.5(\mathrm{ArCH}), 123.4(\mathrm{Ar} \mathrm{CH}), 125.4(\mathrm{ArCH}), 128.5(\mathrm{ArCH}), 130.6$ $(\operatorname{Ar} C), 134.7(\operatorname{Ar} C), 149.8(\operatorname{Ar} C)$.

The data is in accordance with the literature. ${ }^{14}$

\section{2-(2,4-Dimethylphenyl)-1-methyl-1H-pyrrole, 10}<smiles>Nc1ccc(-c2cccn2-c2ccccc2)c(N)c1</smiles>

10

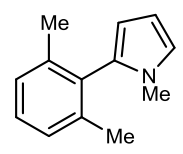

$10^{\prime}$

Prepared as described in General Procedure C: meta-xylene $(25 \mu \mathrm{L}, 0.20 \mathrm{mmol})$, dibenzothiophene $S$ oxide (44 mg, $0.22 \mathrm{mmol}$ ), Tf $2 \mathrm{O}(41 \mu \mathrm{L}, 0.24 \mathrm{mmol}$ ), PTH (5.5 mg, $0.02 \mathrm{mmol}), \mathrm{N}$-methylpyrrole (0.36 $\mathrm{mL}, 4.00 \mathrm{mmol}, 20$ equiv.), after purification by column chromatography on silica gel $\left(20 \% \mathrm{CHCl}_{3}\right.$ in hexanes), gave 10 (isolated as a 15:1 mixture with 10', $13.3 \mathrm{mg}, 0.10 \mathrm{mmol}, 51 \%$ ) as a colourless oil; $10 \delta_{\mathrm{H}}\left(400 \mathrm{MHz}, \mathrm{CDCl}_{3}\right) \delta 2.17\left(\mathrm{~s}, 3 \mathrm{H}, \mathrm{CH}_{3}\right), 2.37\left(\mathrm{~s}, 3 \mathrm{H}, \mathrm{CH}_{3}\right), 3.41\left(\mathrm{~s}, 3 \mathrm{H}, \mathrm{NCH}_{3}\right), 6.05(\mathrm{dd}, J=3.5,1.8$ $\mathrm{Hz}, 1 \mathrm{H}, \operatorname{Ar} H), 6.21(\mathrm{dd}, J=3.5,2.7 \mathrm{~Hz}, 1 \mathrm{H}, \operatorname{Ar} H), 6.71(\mathrm{dd}, J=2.7,1.8 \mathrm{~Hz}, 1 \mathrm{H}, \operatorname{Ar} H), 7.01-7.07(\mathrm{~m}$, $1 \mathrm{H}, \operatorname{Ar} H), 7.11(\mathrm{~s}, 1 \mathrm{H}, \operatorname{Ar} H), 7.14(\mathrm{~d}, J=7.7 \mathrm{~Hz}, 1 \mathrm{H}, \operatorname{Ar} H) ; 10 \delta_{\mathrm{C}}\left(100 \mathrm{MHz}, \mathrm{CDCl}_{3}\right) 20.1\left(\mathrm{CH}_{3}\right), 21.3$ $\left(\mathrm{CH}_{3}\right), 34.2\left(\mathrm{NCH}_{3}\right), 107.3(\mathrm{Ar} \mathrm{CH}), 108.5(\mathrm{Ar} \mathrm{CH}), 121.6(\mathrm{Ar} \mathrm{CH}), 126.3(\mathrm{ArCH}), 130.3(\mathrm{Ar} \mathrm{CH}), 130.9(\mathrm{Ar}$ $C H), 131.3(\operatorname{ArC}), 133.4(\operatorname{ArC}), 137.8(\operatorname{ArC}), 138.2(\operatorname{ArC}) ; v_{\max }\left(\operatorname{thin}\right.$ film/ $\left./ \mathrm{cm}^{-1}\right)$ 821, 883, 981, 1036, 1051, 1087, 1233, 1242, 1357, 1408, 1467, 2859, 2918, 3003; MS (GCMS) m/z $185.1\left(\mathrm{M}^{+}\right)$; HRMS $\mathrm{C}_{13} \mathrm{H}_{16} \mathrm{~N}^{+}\left(\mathrm{M}+\mathrm{H}^{+}\right)$Expected 186.1277, Found 186.1277. 


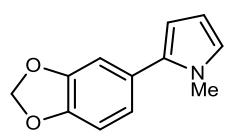

Prepared as described in General Procedure C: 1,3-Benzodioxole (23 $\mu \mathrm{L}, 0.20 \mathrm{mmol}$ ), dibenzothiophene $S$-oxide ( $44 \mathrm{mg}, 0.22 \mathrm{mmol}$ ), $\mathrm{Tf}_{2} \mathrm{O}$ ( $41 \mu \mathrm{L}, 0.24 \mathrm{mmol}$ ), PTH (5.5 mg, $0.02 \mathrm{mmol}$ ), $\mathrm{N}$ methylpyrrole $(0.36 \mathrm{~mL}, 4.00 \mathrm{mmol}, 20$ equiv.), after purification by column chromatography on silica gel (20\% $\mathrm{CHCl}_{3}$ in hexanes), gave $11(24.9 \mathrm{mg}, 0.12 \mathrm{mmol}, 62 \%)$ as a colourless oil; $\delta_{\mathrm{H}}(400 \mathrm{MHz}$, $\left.\mathrm{CDCl}_{3}\right) 3.64\left(\mathrm{~s}, 3 \mathrm{H}, \mathrm{CH}_{3}\right), 6.00\left(\mathrm{~s}, 2 \mathrm{H}, \mathrm{CH}_{2}\right), 6.16(\mathrm{dd}, J=3.6,1.9 \mathrm{~Hz}, 1 \mathrm{H}, \operatorname{Ar} \mathrm{H}), 6.19$ (dd, J = 3.6, $2.7 \mathrm{~Hz}$, $1 \mathrm{H}, \operatorname{Ar} H), 6.69(\mathrm{dd}, J=2.7,1.9 \mathrm{~Hz}, 1 \mathrm{H}, \operatorname{Ar} H), 6.85-6.87(\mathrm{~m}, 2 \mathrm{H}, \operatorname{Ar} H), 6.88-6.89(\mathrm{~m}, 1 \mathrm{H}, \operatorname{Ar} H) ; \delta_{c}$ (100 MHz, $\left.\mathrm{CDCl}_{3}\right) 35.0\left(\mathrm{CH}_{3}\right), 101.2\left(\mathrm{CH}_{2}\right), 107.7(\mathrm{Ar} \mathrm{CH}), 108.4(\mathrm{ArCH}), 108.4(\mathrm{ArCH}), 109.5(\mathrm{Ar} \mathrm{CH})$, 122.5 ( $\mathrm{ArCH}), 123.3(\operatorname{ArCH}), 127.4(\operatorname{ArC}), 134.4(\operatorname{ArC}), 146.8(\operatorname{ArC}), 147.7(\operatorname{ArC}) ; v_{\max }\left(\operatorname{thin} \mathrm{film} / \mathrm{cm}^{-1}\right)$ 781, 811, 868, 987, 1036, 1124, 1221, 1242, 1305, 1331, 1435, 1450, 2777, 2888; MS (GCMS) m/z $201.1\left(\mathrm{M}^{+}\right)$; HRMS $\mathrm{C}_{12} \mathrm{H}_{12} \mathrm{O}_{2} \mathrm{~N}^{+}\left(\mathrm{M}+\mathrm{H}^{+}\right)$Expected 202.0863, Found 202.0863.

1-Methyl-2-(4-phenoxyphenyl)-1H-pyrrole, 12

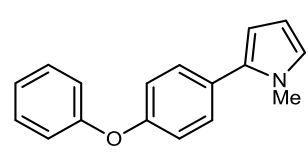

12

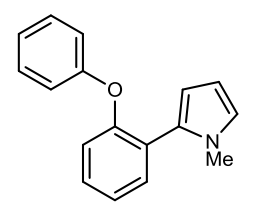

$12^{\prime}$

Prepared as described in General Procedure C: diphenyl ether (32 $\mu \mathrm{L}, 0.20 \mathrm{mmol})$, dibenzothiophene S-oxide (44 mg, $0.22 \mathrm{mmol}$ ), Tf $\mathrm{Tf}_{2} \mathrm{O}(41 \mu \mathrm{L}, 0.24 \mathrm{mmol}$ ), PTH (5.5 mg, $0.02 \mathrm{mmol}$ ), $\mathrm{N}$-methylpyrrole ( $0.36 \mathrm{~mL}, 4.00 \mathrm{mmol}, 20$ equiv.), after purification by column chromatography on silica gel (20\% $\mathrm{CHCl}_{3}$ in hexanes), gave 12 (isolated as an 11:1 mixture with 12 ', $33.4 \mathrm{mg}, 0.13 \mathrm{mmol}, 67 \%$ ) as a colourless oil; $12 \delta_{\mathrm{H}}\left(400 \mathrm{MHz}, \mathrm{CDCl}_{3}\right) 3.67\left(\mathrm{~s}, 3 \mathrm{H}, \mathrm{CH}_{3}\right), 6.22(\mathrm{~d}, J=2.3 \mathrm{~Hz}, 2 \mathrm{H}, \mathrm{Ar} \mathrm{H}), 6.72(\mathrm{t}, J=2.3$ $\mathrm{Hz}, 1 \mathrm{H}, \operatorname{Ar} H), 7.05(\mathrm{~d}, J=8.7 \mathrm{~Hz}, 2 \mathrm{H}, \operatorname{Ar} H), 7.06-7.10(\mathrm{~m}, 2 \mathrm{H}, \operatorname{Ar} H), 7.12-7.18(\mathrm{~m}, 1 \mathrm{H}, \operatorname{Ar} H), 7.33-$ $7.42(\mathrm{~m}, 4 \mathrm{H}, \mathrm{Ar} \mathrm{H}) ; 12 \delta_{\mathrm{C}}\left(100 \mathrm{MHz}, \mathrm{CDCl}_{3}\right) 35.1\left(\mathrm{CH}_{3}\right), 107.8(\mathrm{ArCH}), 108.5(\mathrm{ArCH}), 118.7(\mathrm{Ar} \mathrm{CH})$, 119.2 ( $\mathrm{ArCH}), 123.5(\mathrm{ArCH}), 123.6(\mathrm{ArCH}), 128.5(\mathrm{ArC}), 129.9(\mathrm{ArCH}), 130.2(\mathrm{ArCH}), 134.1(\mathrm{ArC})$, $156.5(\operatorname{ArC}), 157.1(\mathrm{ArCH}) ; v_{\max }\left(\right.$ thin film/ $\mathrm{cm}^{-1}$ ) 839, 868, 982, 1022, 1056, 1166, 1228, 1308, 1475, 1487, 1588, 2922, 3038; MS (GCMS) $\mathrm{m} / 2249.1\left(\mathrm{M}^{+}\right)$; HRMS $\mathrm{C}_{17} \mathrm{H}_{16} \mathrm{ON}^{+}\left(\mathrm{M}+\mathrm{H}^{+}\right)$Expected 250.1226, Found 250.1223. 
<smiles></smiles>

Prepared as described in General Procedure C: $\mathbf{S 7}$ (36 mg, $0.20 \mathrm{mmol}$ ), dibenzothiophene S-oxide (44 $\mathrm{mg}, 0.22 \mathrm{mmol}), \mathrm{Tf}_{2} \mathrm{O}(41 \mu \mathrm{L}, 0.24 \mathrm{mmol})$, PTH $(5.5 \mathrm{mg}, 0.02 \mathrm{mmol}), \mathrm{N}$-methylpyrrole $(0.36 \mathrm{~mL}, 4.00$ mmol, 20 equiv.), after purification by column chromatography on silica gel ( $70 \% \mathrm{CHCl}_{3}$ in hexanes), gave $13(26.2 \mathrm{mg}, 0.10 \mathrm{mmol}, 51 \%)$ as a white gum; $\delta_{\mathrm{H}}\left(400 \mathrm{MHz}, \mathrm{CDCl}_{3}\right) 1.38\left(\mathrm{~s}, 9 \mathrm{H}, \mathrm{CH}_{3}\right), 3.66(\mathrm{~s}, 3 \mathrm{H}$, $\left.\mathrm{NCH}_{3}\right), 6.17-6.25(\mathrm{~m}, 2 \mathrm{H}, \operatorname{Ar} H), 6.68-6.75(\mathrm{~m}, 1 \mathrm{H}, \operatorname{Ar} H), 7.10(\mathrm{~d}, J=8.6 \mathrm{~Hz}, 2 \mathrm{H}, \operatorname{Ar} H), 7.40(\mathrm{~d}, J=$ 8.6 Hz, 2H, $\mathrm{Ar} \mathrm{H})$; $\delta_{\mathrm{C}}\left(100 \mathrm{MHz}, \mathrm{CDCl}_{3}\right) 27.3\left(\mathrm{CH}_{3}\right), 35.1\left(\mathrm{NCH}_{3}\right), 39.3\left(\mathrm{CH}_{3} \mathrm{C}\right), 107.9(\mathrm{ArCH}), 108.9(\mathrm{Ar}$ $\mathrm{CH}), 121.5(\operatorname{ArCH}), 123.8(\operatorname{Ar} C H), 129.7(\operatorname{ArCH}), 131.0(\operatorname{Ar} C), 133.9(\operatorname{ArC} C), 150.1(\operatorname{Ar} C), 177.3(C=0)$; $v_{\max }\left(\right.$ thin film/cm $\left.{ }^{-1}\right) 710,780,851,942,1014,1028,1112,1165,1198,1277,1309,1476,1501,1546$, 1745, 2873, 2932, 2972; MS (GCMS) m/z $257.1\left(\mathrm{M}^{+}\right)$; HRMS $\mathrm{C}_{16} \mathrm{H}_{20} \mathrm{O}_{2} \mathrm{~N}^{+}\left(\mathrm{M}+\mathrm{H}^{+}\right)$Expected 258.1489, Found 258.1488.

1-(4-(1-Methyl-1H-pyrrol-2-yl)phenyl)pyrrolidin-2-one, 14

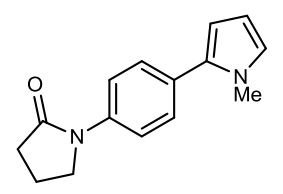

Prepared as described in General Procedure D: 1-phenyl-2-pyrrolidinone (32 mg, $0.20 \mathrm{mmol}$ ), dibenzothiophene $S$-oxide (44 mg, $0.22 \mathrm{mmol}$ ), $\mathrm{Tf}_{2} \mathrm{O}$ (41 $\mu \mathrm{L}, 0.24 \mathrm{mmol}$ ), PTH (5.5 mg, $\left.0.02 \mathrm{mmol}\right), \mathrm{N}$ methylpyrrole ( $0.36 \mathrm{~mL}, 4.00 \mathrm{mmol}, 20$ equiv.), after purification by column chromatography on silica gel (20\% EtOAc in hexanes), gave $14(28.8 \mathrm{mg}, 0.12 \mathrm{mmol}, 60 \%)$ as a white solid; m.p. (recrystallized from $\left.\mathrm{CHCl}_{3}\right) 119-12{ }^{\circ} \mathrm{C} ; \delta_{\mathrm{H}}\left(400 \mathrm{MHz}, \mathrm{CDCl}_{3}\right) 2.18\left(\mathrm{tt}, J=7.7,6.8 \mathrm{~Hz}, 2 \mathrm{H}, \mathrm{CH}_{2}\right), 2.63$ $\left(\mathrm{dd}, J=8.5,7.7 \mathrm{~Hz}, 2 \mathrm{H}, \mathrm{CH}_{2} \mathrm{C}(\mathrm{O})\right), 3.66\left(\mathrm{~s}, 3 \mathrm{H}, \mathrm{CH}_{3}\right), 3.85-3.94\left(\mathrm{~m}, 2 \mathrm{H}, \mathrm{CH}_{2} \mathrm{~N}\right), 6.17-6.24(\mathrm{~m}, 2 \mathrm{H}, \mathrm{Ar}$ H), $6.71(\mathrm{dd}, J=2.6,1.9 \mathrm{~Hz}, 1 \mathrm{H}, \operatorname{Ar} H), 7.41(\mathrm{~d}, J=8.8 \mathrm{~Hz}, 2 \mathrm{H}, \operatorname{Ar} H), 7.65(\mathrm{~d}, J=8.8 \mathrm{~Hz}, 2 \mathrm{H}, \operatorname{Ar} H) ; \delta_{\mathrm{C}}$ (100 MHz, CDCl $)$ 18.2 $\left(\mathrm{CH}_{2}\right), 32.9\left(\mathrm{CH}_{2} \mathrm{C}(\mathrm{O})\right)$, $35.2\left(\mathrm{CH}_{3}\right), 48.9\left(\mathrm{CH}_{2} \mathrm{~N}\right), 107.9(\mathrm{ArCH}), 108.7(\mathrm{ArCH})$, 119.9 (Ar CH), 123.7 ( $\operatorname{ArCH}), 129.1(\operatorname{ArCH}), 129.6(\operatorname{ArC}), 134.2(\operatorname{ArC}), 138.2(\operatorname{ArC}), 174.3(C=0) ; v_{\max }$ (thin film/cm ${ }^{-1}$ ) 838, 982, 1024, 1057, 1125, 1222, 1243, 1389, 1477, 1504, 1547, 1688, 2878, 2975, 3098; MS (GCMS) m/z 240.1 (M+); $\mathrm{HRMS} \mathrm{C}_{15} \mathrm{H}_{17} \mathrm{ON}_{2}{ }^{+}\left(\mathrm{M}+\mathrm{H}^{+}\right)$Expected 241.1335, Found 241.1337. 
N-(4-(1-Methyl-1H-pyrrol-2-yl)phenyl)acetamide, 15<smiles></smiles>

Prepared as described in General Procedure D: $N$-acetylaniline (27 mg, $0.20 \mathrm{mmol}$ ), dibenzothiophene S-oxide (44 mg, $0.22 \mathrm{mmol}$ ), $\mathrm{Tf}_{2} \mathrm{O}$ (41 $\mu \mathrm{L}, 0.24 \mathrm{mmol}$ ), PTH (5.5 mg, $0.02 \mathrm{mmol}$ ), $N$ methylpyrrole $(0.36 \mathrm{~mL}, 4.00 \mathrm{mmol}, 20$ equiv.), after purification by column chromatography on silica gel (50\% EtOAc in hexanes), gave $15(23.1 \mathrm{mg}, 0.11 \mathrm{mmol}, 54 \%)$ as a white solid; m.p. (recrystallized from $\left.\mathrm{CHCl}_{3}\right) 164-166{ }^{\circ} \mathrm{C} ; \delta_{\mathrm{H}}\left(400 \mathrm{MHz}, \mathrm{CDCl}_{3}\right) 2.19\left(\mathrm{~s}, 3 \mathrm{H}, \mathrm{CH}_{3}\right), 3.64\left(\mathrm{~s}, 3 \mathrm{H}, \mathrm{NCH}_{3}\right)$, $6.16-6.23(\mathrm{~m}, 2 \mathrm{H}, \operatorname{Ar} H), 6.67-6.73(\mathrm{~m}, 1 \mathrm{H}, \operatorname{Ar} H), 7.35(\mathrm{br} \mathrm{s}, \mathrm{NH}), 7.35(\mathrm{~d}, J=8.6 \mathrm{~Hz}, 2 \mathrm{H}, \operatorname{Ar} H), 7.53$ $(\mathrm{d}, J=8.6 \mathrm{~Hz}, 2 \mathrm{H}, \mathrm{Ar} \mathrm{H}) ; \delta_{\mathrm{C}}\left(100 \mathrm{MHz}, \mathrm{CDCl}_{3}\right) 24.8\left(\mathrm{CH}_{3}\right), 35.2\left(\mathrm{NCH}_{3}\right), 107.9(\mathrm{ArCH}), 108.6(\mathrm{ArCH})$, 120.0 (Ar CH), 123.7 ( $\operatorname{ArCH}), 129.3(\operatorname{ArCH}), 129.6(\operatorname{ArC} C), 134.2(\operatorname{ArC}), 136.7(\operatorname{ArC}), 168.4(C=0) ; v_{\max }$ (thin film/cm ${ }^{-1}$ ) 788, 832, 966, 978, 1017, 1183, 1238, 1265, 1321, 1366, 1401, 1505, 1529, 1542, 1601, 1658, 2927, 3061, 3116, 3182, 3303; MS (GCMS) m/z $214.1\left(\mathrm{M}^{+}\right) ; \mathrm{HRMS} \mathrm{C}_{13} \mathrm{H}_{15} \mathrm{ON}_{2}{ }^{+}\left(\mathrm{M}^{+} \mathrm{H}^{+}\right)$ Expected 215.1179, Found 215.1177.

4-Methyl-N-(4-(1-methyl-1H-pyrrol-2-yl)phenyl)benzenesulfonamide, 16

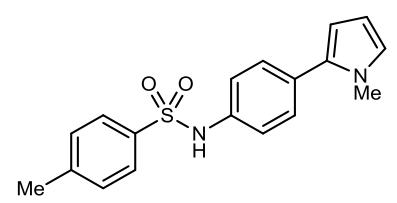

Prepared as described in General Procedure D: $N$-tosylaniline $(50 \mathrm{mg}, 0.20 \mathrm{mmol})$, dibenzothiophene S-oxide (44 mg, $0.22 \mathrm{mmol}$ ), Tf $\mathrm{T}_{2} \mathrm{O}(41 \mu \mathrm{L}, 0.24 \mathrm{mmol}$ ), PTH (5.5 mg, $0.02 \mathrm{mmol}$ ), $N$-methylpyrrole ( $0.36 \mathrm{~mL}, 4.00 \mathrm{mmol}, 20$ equiv.), after purification by column chromatography on silica gel ( $25 \%$ EtOAc in hexanes), gave 16 (37.9 g, $0.12 \mathrm{mmol}, 58 \%)$ as a white solid; m.p. (recrystallized from $\mathrm{CHCl}_{3}$ ) $86-88^{\circ} \mathrm{C} ; \delta_{\mathrm{H}}\left(400 \mathrm{MHz}, \mathrm{CDCl}_{3}\right) 2.39\left(\mathrm{~s}, 3 \mathrm{H}, \mathrm{CH}_{3}\right), 3.61\left(\mathrm{~s}, 3 \mathrm{H}, \mathrm{NCH}_{3}\right), 6.13-6.21(\mathrm{~m}, 2 \mathrm{H}, \mathrm{Ar} \mathrm{H}), 6.66-$ $6.72(\mathrm{~m}, 1 \mathrm{H}, \operatorname{Ar} H), 6.87(\mathrm{~s}, 1 \mathrm{H}, \mathrm{NH}), 7.10(\mathrm{~d}, J=8.4 \mathrm{~Hz}, 2 \mathrm{H}, \operatorname{Ar} H), 7.23-7.31(\mathrm{~m}, 4 \mathrm{H}, \operatorname{Ar} H), 7.71(\mathrm{~d}, J$ $=8.4 \mathrm{~Hz}, 2 \mathrm{H}, \operatorname{Ar} H) ; \delta_{\mathrm{C}}\left(100 \mathrm{MHz}, \mathrm{CDCl}_{3}\right) 21.7\left(\mathrm{CH}_{3}\right), 35.2\left(\mathrm{NCH}_{3}\right), 108.0(\operatorname{ArCH}), 108.8(\operatorname{ArCH}), 121.6$ ( $\operatorname{ArCH}), 124.0(\operatorname{ArCH}), 127.4(\operatorname{ArCH}), 129.5(\operatorname{ArCH}), 129.8(\operatorname{ArCH}), 130.6(\operatorname{ArC}), 133.7(\operatorname{ArC}), 135.2$ $(\operatorname{ArC}), 136.3(\operatorname{ArC}), 144.1(\operatorname{ArC}) ; v_{\max }\left(\operatorname{thin}\right.$ film $\left./ \mathrm{cm}^{-1}\right)$ 813, 912, 1017, 1089, 1155, 1223, 1328, 1393, $1447,1479,1547,1596,2922,3046,3249 ; \mathrm{MS}(\mathrm{GCMS}) \mathrm{m} / \mathrm{z} 326.1\left(\mathrm{M}^{+}\right) ; \mathrm{HRMS} \mathrm{C}_{18} \mathrm{H}_{19} \mathrm{O}_{2} \mathrm{~N}_{2} \mathrm{~S}^{+}\left(\mathrm{M}+\mathrm{H}^{+}\right)$ Expected 327.1162, Found 327.1153. 
N-(4-(1-Methyl-1H-pyrrol-2-yl)phenyl)methanesulfonamide, 17

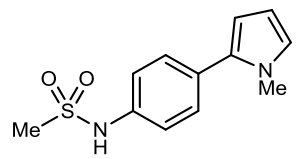

Prepared as described in General Procedure D: $N$-mesylaniline (34 mg, $0.20 \mathrm{mmol}$ ), dibenzothiophene S-oxide (44 mg, $0.22 \mathrm{mmol}$ ), $\mathrm{Tf}_{2} \mathrm{O}$ (41 $\mu \mathrm{L}, 0.24 \mathrm{mmol}$ ), PTH (5.5 mg, $\left.0.02 \mathrm{mmol}\right), \mathrm{N}$ methylpyrrole $(0.36 \mathrm{~mL}, 4.00 \mathrm{mmol}, 20$ equiv.), after purification by column chromatography on silica gel (30\% EtOAc in hexanes), gave $17(28.0 \mathrm{mg}, 0.11 \mathrm{mmol}, 56 \%)$ as a colourless oil; $\delta_{\mathrm{H}}(400$ $\left.\mathrm{MHz}, \mathrm{CDCl}_{3}\right) 3.07\left(\mathrm{~s}, 3 \mathrm{H}, \mathrm{CH}_{3}\right), 3.66\left(\mathrm{~s}, 3 \mathrm{H}, \mathrm{NCH}_{3}\right), 6.17-6.25(\mathrm{~m}, 2 \mathrm{H}, \mathrm{ArH}), 6.69-6.75(\mathrm{~m}, 1 \mathrm{H}, \mathrm{Ar} \mathrm{H})$, $6.78(\mathrm{~s}, 1 \mathrm{H}, \mathrm{NH}), 7.27(\mathrm{~d}, J=8.5 \mathrm{~Hz}, 2 \mathrm{H}, \operatorname{Ar} H), 7.39(\mathrm{~d}, J=8.5 \mathrm{~Hz}, 2 \mathrm{H}, \operatorname{Ar} H) ; \delta_{\mathrm{c}}\left(100 \mathrm{MHz}, \mathrm{CDCl}_{3}\right) 35.2$ $\left(\mathrm{NCH}_{3}\right), 39.7\left(\mathrm{CH}_{3}\right), 108.0(\mathrm{ArCH}), 109.0(\mathrm{ArCH}), 120.9(\mathrm{ArCH}), 124.1(\mathrm{ArCH}), 129.9(\mathrm{ArCH}), 130.9(\mathrm{Ar}$ C), $133.6(\operatorname{ArC}), 135.3(\operatorname{ArC}) ; v_{\max }\left(\right.$ thin film $\left./ \mathrm{cm}^{-1}\right)$ 840, 907, 966, 1058, 1090, 1147, 1222, 1322, 1391, 1504, 1546, 2849, 2927, 3012, 3257; MS (GCMS) $\mathrm{m} / \mathrm{z} 250.1\left(\mathrm{M}^{+}\right) ; \operatorname{HRMS} \mathrm{C}_{12} \mathrm{H}_{15} \mathrm{O}_{2} \mathrm{~N}_{2} \mathrm{~S}^{+}\left(\mathrm{M}+\mathrm{H}^{+}\right)$ Expected 251.0849, Found 251.0848.

\section{2-(3-Fluoro-4-methoxyphenyl)-1-methyl-1H-pyrrole, 18}<smiles></smiles>

Prepared as described in General Procedure C: 2-fluoroanisole (23 $\mu \mathrm{L}, 0.20 \mathrm{mmol}$ ), dibenzothiophene $S$-oxide (44 mg, $0.22 \mathrm{mmol}$ ), $\mathrm{Tf}_{2} \mathrm{O}$ (41 $\mu \mathrm{L}, 0.24 \mathrm{mmol}$ ), PTH (5.5 mg, $\left.0.02 \mathrm{mmol}\right), \mathrm{N}$ methylpyrrole $(0.36 \mathrm{~mL}, 4.00 \mathrm{mmol}, 20$ equiv.), after purification by column chromatography on silica gel (30\% $\mathrm{CHCl}_{3}$ in hexanes), gave $18(27.5 \mathrm{mg}, 0.13 \mathrm{mmol}, 67 \%)$ as a white solid; m.p. (recrystallized from $\left.\mathrm{CHCl}_{3}\right) 53-55^{\circ} \mathrm{C} ; \delta_{\mathrm{H}}\left(400 \mathrm{MHz}, \mathrm{CDCl}_{3}\right) 3.65\left(\mathrm{~s}, 3 \mathrm{H}, \mathrm{NCH}_{3}\right), 3.93\left(\mathrm{~s}, 3 \mathrm{H}, \mathrm{OCH}_{3}\right), 6.15$ - $6.23(\mathrm{~m}, 2 \mathrm{H}, \operatorname{Ar} H), 6.68-6.73(\mathrm{~m}, 1 \mathrm{H}, \operatorname{Ar} H), 6.95-7.04(\mathrm{~m}, 1 \mathrm{H}, \operatorname{Ar} H), 7.09-7.17(\mathrm{~m}, 2 \mathrm{H}, \operatorname{Ar} H) ; \delta_{\mathrm{C}}$ $\left(100 \mathrm{MHz}, \mathrm{CDCl}_{3}\right) 35.1\left(\mathrm{NCH}_{3}\right), 56.5\left(\mathrm{OCH}_{3}\right), 107.9(\mathrm{ArCH}), 108.7(\mathrm{ArCH}), 113.4(\mathrm{~d}, \mathrm{~J}=2.4 \mathrm{~Hz}, \mathrm{ArCH})$, $116.6(\mathrm{~d}, J=18.8 \mathrm{~Hz}, \operatorname{Ar} \mathrm{CH}), 123.7(\operatorname{ArCH}), 124.6(\mathrm{~d}, J=3.4 \mathrm{~Hz}, \operatorname{Ar} C H), 126.7$ (d, J = 7.0 Hz, $\operatorname{Ar} C$ ), $133.3(\mathrm{~d}, J=2.0 \mathrm{~Hz}, \operatorname{Ar} C), 146.7$ (d, $J=10.7 \mathrm{~Hz}, \operatorname{Ar} C), 152.1(\mathrm{~d}, J=245.7 \mathrm{~Hz}, \operatorname{Ar} C) ; \delta_{\mathrm{F}}(376 \mathrm{MHz}$, $\mathrm{CDCl}_{3}$ ) -135.3; $v_{\max }$ (thin film/cm ${ }^{-1}$ ) 813, 874, 996, 1023, 1049, 1090, 1169, 1268, 1297, 1463, 1478, 1508, 1549, 2838, 2934, 3098; MS (GCMS) $\mathrm{m} / \mathrm{z} 205.0\left(\mathrm{M}^{+}\right)$; HRMS $\mathrm{C}_{12} \mathrm{H}_{13} \mathrm{ONF}^{+}\left(\mathrm{M}+\mathrm{H}^{+}\right)$Expected 206.0976, Found 206.0975. 


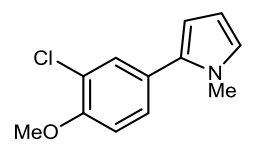

Prepared as described in General Procedure C: 2-chloroanisole (25 $\mu \mathrm{L}, 0.20 \mathrm{mmol})$, dibenzothiophene $S$-oxide (44 mg, $0.22 \mathrm{mmol}$ ), $\mathrm{Tf}_{2} \mathrm{O}$ ( $41 \mu \mathrm{L}, 0.24 \mathrm{mmol}$ ), PTH (5.5 mg, $\left.0.02 \mathrm{mmol}\right), \mathrm{N}$ methylpyrrole $(0.36 \mathrm{~mL}, 4.00 \mathrm{mmol}, 20$ equiv.), after purification by column chromatography on silica gel (40\% $\mathrm{CHCl}_{3}$ in hexanes), gave $19(30.2 \mathrm{mg}, 0.14 \mathrm{mmol}, 68 \%)$ as an off-white solid; m.p. (recrystallized from $\left.\mathrm{CHCl}_{3}\right) 58-60^{\circ} \mathrm{C} ; \delta_{\mathrm{H}}\left(400 \mathrm{MHz}, \mathrm{CDCl}_{3}\right) 3.64\left(\mathrm{~s}, 3 \mathrm{H}, \mathrm{NCH}_{3}\right), 3.94\left(\mathrm{~s}, 3 \mathrm{H}, \mathrm{OCH}_{3}\right), 6.15$ - $6.21(\mathrm{~m}, 2 \mathrm{H}, \operatorname{Ar} H), 6.67-6.73(\mathrm{~m}, 1 \mathrm{H}, \operatorname{Ar} H), 6.97(\mathrm{~d}, J=8.5 \mathrm{~Hz}, 1 \mathrm{H}, \operatorname{Ar} H), 7.26(\mathrm{dd}, J=8.5,2.2 \mathrm{~Hz}$, $1 \mathrm{H}, \operatorname{Ar} H), 7.42(\mathrm{~d}, J=2.2 \mathrm{~Hz}, 1 \mathrm{H}, \operatorname{Ar} H) ; \delta_{\mathrm{C}}\left(100 \mathrm{MHz}, \mathrm{CDCl}_{3}\right) 35.1\left(\mathrm{NCH}_{3}\right), 56.3\left(\mathrm{OCH}_{3}\right), 107.9(\mathrm{ArCH})$, 108.7 ( $\mathrm{ArCH}), 112.0(\operatorname{ArCH}), 122.4(\operatorname{ArC}), 123.6(\operatorname{ArCH}), 127.0(\operatorname{ArC}), 128.2(\operatorname{ArCH}), 130.6(\operatorname{Ar} C H)$, $133.1(\operatorname{ArC}), 154.1(\operatorname{ArC}) ; v_{\max }\left(\right.$ thin film $/ \mathrm{cm}^{-1}$ ) 885, 992, 1020, 1061, 1149, 1183, 1253, 1287, 1474, $1498,1544,1564,2837,2942,3002,3098 ; \mathrm{MS}$ (GCMS) $\mathrm{m} / \mathrm{z} 221.0\left(\mathrm{M}^{+}\right) ; \mathrm{HRMS} \mathrm{C}_{12} \mathrm{H}_{13} \mathrm{ONCl}^{+}\left(\mathrm{M}^{+} \mathrm{H}^{+}\right)$ Expected 222.0680, Found 222.0681.

2-(3-Bromo-4-methoxyphenyl)-1-methyl-1H-pyrrole, 20

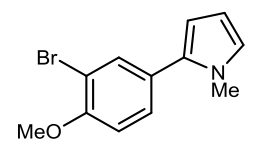

Prepared as described in General Procedure C: 2-bromoanisole (25 $\mu \mathrm{L}, 0.20 \mathrm{mmol}$ ), dibenzothiophene $S$-oxide (44 mg, $0.22 \mathrm{mmol}$ ), $\mathrm{Tf}_{2} \mathrm{O}$ (41 $\left.\mu \mathrm{L}, 0.24 \mathrm{mmol}\right), \mathrm{PTH}(5.5 \mathrm{mg}, 0.02 \mathrm{mmol}), \mathrm{N}$ methylpyrrole $(0.36 \mathrm{~mL}, 4.00 \mathrm{mmol}, 20$ equiv.), after purification by column chromatography on silica gel (30\% $\mathrm{CHCl}_{3}$ in hexanes), gave $20(36.6 \mathrm{mg}, 0.14 \mathrm{mmol}, 69 \%)$ as an off-white solid; m.p. (recrystallized from $\left.\mathrm{CHCl}_{3}\right) 71-73^{\circ} \mathrm{C} ; \delta_{\mathrm{H}}\left(400 \mathrm{MHz}, \mathrm{CDCl}_{3}\right) 3.64\left(\mathrm{~s}, 3 \mathrm{H}, \mathrm{NCH}_{3}\right), 3.93\left(\mathrm{~s}, 3 \mathrm{H}, \mathrm{OCH}_{3}\right), 6.13$ - $6.22(\mathrm{~m}, 2 \mathrm{H}, \operatorname{Ar} H), 6.67-6.73(\mathrm{~m}, 1 \mathrm{H}, \operatorname{Ar} H), 6.94(\mathrm{~d}, J=8.4 \mathrm{~Hz}, 1 \mathrm{H}, \operatorname{Ar} H), 7.31(\mathrm{dd}, J=8.4,2.2 \mathrm{~Hz}$, $1 \mathrm{H}, \operatorname{Ar} H), 7.60(\mathrm{~d}, J=2.2 \mathrm{~Hz}, 1 \mathrm{H}, \mathrm{Ar} H) ; \delta_{\mathrm{C}}\left(100 \mathrm{MHz}, \mathrm{CDCl}_{3}\right) 35.0\left(\mathrm{NCH}_{3}\right), 56.4\left(\mathrm{OCH}_{3}\right), 107.9(\mathrm{ArCH})$, 108.7 ( $\mathrm{ArCH}), 111.6(\operatorname{ArC}), 111.8(\operatorname{ArCH}), 123.6(\operatorname{ArCH}), 127.5(\operatorname{ArC}), 128.9(\operatorname{ArCH}), 132.9(\operatorname{ArC})$, 133.6 (Ar CH), $155.0(\operatorname{Ar} C) ; v_{\max }\left(\right.$ thin film/ $\left.\mathrm{cm}^{-1}\right)$ 711, 783, 812, 885, 1017, 1053, 1147, 1182, 1251, $1283,1360,1441,1542,2836,2939,3002 ; \mathrm{MS}$ (GCMS) $\mathrm{m} / \mathrm{z} 265.0\left(\mathrm{M}^{+}\right) ; \mathrm{HRMS} \mathrm{C}_{12} \mathrm{H}_{13} \mathrm{ONBr}^{+}\left(\mathrm{M}^{+} \mathrm{H}^{+}\right)$ Expected 266.0175, Found 266.0177. 
<smiles></smiles>

Prepared as described in General Procedure C: 2-iodoanisole ( $26 \mu \mathrm{L}, 0.20 \mathrm{mmol}$ ), dibenzothiophene S-oxide (44 mg, $0.22 \mathrm{mmol}$ ), Tf $\mathrm{O}_{2} \mathrm{O}(41 \mu \mathrm{L}, 0.24 \mathrm{mmol}$ ), PTH (5.5 mg, $0.02 \mathrm{mmol}$ ), $\mathrm{N}$-methylpyrrole $(0.36 \mathrm{~mL}, 4.00 \mathrm{mmol}, 20$ equiv.), after purification by column chromatography on silica gel (30\% $\mathrm{CHCl}_{3}$ in hexanes), gave 21 (40.1 mg, $0.13 \mathrm{mmol}, 64 \%$ ) as a colourless oil; $\delta_{\mathrm{H}}\left(400 \mathrm{MHz}, \mathrm{CDCl}_{3}\right) 3.63$ (s, $\left.3 \mathrm{H}, \mathrm{NCH}_{3}\right), 3.92\left(\mathrm{~s}, 3 \mathrm{H}, \mathrm{OCH}_{3}\right), 6.12-6.24(\mathrm{~m}, 2 \mathrm{H}, \operatorname{Ar} H), 6.67-6.73(\mathrm{~m}, 1 \mathrm{H}, \operatorname{Ar} H), 6.86(\mathrm{~d}, J=8.5 \mathrm{~Hz}$, $1 \mathrm{H}, \operatorname{Ar} H), 7.34(\mathrm{dd}, J=8.5,2.1 \mathrm{~Hz}, 1 \mathrm{H}, \operatorname{Ar} H), 7.84(\mathrm{~d}, J=2.1 \mathrm{~Hz}, 1 \mathrm{H}, \operatorname{Ar} H) ; \delta_{\mathrm{C}}\left(100 \mathrm{MHz}, \mathrm{CDCl}_{3}\right) 35.0$ $\left(\mathrm{NCH}_{3}\right), 56.6\left(\mathrm{OCH}_{3}\right), 85.9(\operatorname{ArC}), 107.8(\mathrm{Ar} \mathrm{CH}), 108.7(\mathrm{ArCH}), 110.7(\mathrm{ArCH}), 123.6(\mathrm{ArCH}), 128.1(\mathrm{Ar}$ C), $130.0(\operatorname{ArCH}), 132.8(\operatorname{ArC}), 139.8(\mathrm{ArCH}), 157.2(\operatorname{ArC}) ; v_{\max }\left(\right.$ thin film/ $\left./ \mathrm{cm}^{-1}\right)$ 710, 751, 815, 886, $1014,1037,1144,1180,1239,1279,1427,1461,1490,1540,2835,2937,2959$, 3002; MS (GCMS) $\mathrm{m} / \mathrm{z} 313.0\left(\mathrm{M}^{+}\right) ; \mathrm{HRMS} \mathrm{C}_{12} \mathrm{H}_{13} \mathrm{ONI}{ }^{+}\left(\mathrm{M}+\mathrm{H}^{+}\right)$Expected 314.0036, Found 314.0035.

2-Methoxy-5-(1-methyl-1H-pyrrol-2-yl)phenyl trifluoromethanesulfonate, 22<smiles></smiles>

Prepared as described in General Procedure C: 2-methoxyphenyl trifluoromethanesulfonate $(51 \mathrm{mg}$, $0.20 \mathrm{mmol}$ ), dibenzothiophene $\mathrm{S}$-oxide (44 mg, $0.22 \mathrm{mmol}$ ), $\mathrm{Tf}_{2} \mathrm{O}(41 \mu \mathrm{L}, 0.24 \mathrm{mmol}), \mathrm{PTH}(5.5 \mathrm{mg}$, $0.02 \mathrm{mmol}), N$-methylpyrrole $(0.36 \mathrm{~mL}, 4.00 \mathrm{mmol}, 20$ equiv.), after purification by column chromatography on silica gel (40\% $\mathrm{CHCl}_{3}$ in hexanes), gave $22(57.5 \mathrm{mg}, 0.17 \mathrm{mmol}, 86 \%)$ as a colourless oil; $\delta_{\mathrm{H}}\left(400 \mathrm{MHz}, \mathrm{CDCl}_{3}\right) 3.52\left(\mathrm{~s}, 3 \mathrm{H}, \mathrm{NCH}_{3}\right), 3.85\left(\mathrm{~s}, 3 \mathrm{H}, \mathrm{OCH}_{3}\right), 6.20(\mathrm{dd}, \mathrm{J}=3.6,1.8 \mathrm{~Hz}, 1 \mathrm{H}$, $\operatorname{Ar} H), 6.24(\mathrm{dd}, J=3.6,2.7 \mathrm{~Hz}, 1 \mathrm{H}, \operatorname{Ar} H), 6.77(\mathrm{dd}, J=2.7,1.8 \mathrm{~Hz}, 1 \mathrm{H}, \operatorname{Ar} H), 6.97(\mathrm{~d}, J=9.0 \mathrm{~Hz}, 1 \mathrm{H}, \operatorname{Ar}$ H), $7.19-7.29(\mathrm{~m}, 2 \mathrm{H}, \mathrm{Ar} \mathrm{H}) ; \delta_{\mathrm{C}}\left(100 \mathrm{MHz}, \mathrm{CDCl}_{3}\right) 34.8\left(\mathrm{NCH}_{3}\right), 56.1\left(\mathrm{OCH}_{3}\right), 108.0(\mathrm{ArCH}), 110.2(\mathrm{Ar}$ $\mathrm{CH}), 111.6(\mathrm{ArCH}), 118.9$ (q, J = 320.9 Hz, $\left.\mathrm{CF}_{3}\right), 121.6(\operatorname{ArCH}), 123.6(\operatorname{ArCH}), 124.5(\operatorname{ArC}), 124.8(\operatorname{Ar}$ $C H), 129.0(\operatorname{ArC}), 142.8(\operatorname{ArC}), 156.9(\operatorname{ArC}) ; \delta_{\mathrm{F}}\left(376 \mathrm{MHz}, \mathrm{CDCl}_{3}\right)-72.7 ; v_{\max }\left(\right.$ thin film/ $\left.\mathrm{cm}^{-1}\right)$ 714, 878, $906,1025,1089,1135,1204,1418,1495,1546,2844,2915,2942,3002$; MS (GCMS) m/z $335.1\left(M^{+}\right)$; HRMS $\mathrm{C}_{13} \mathrm{H}_{13} \mathrm{O}_{4} \mathrm{NF}_{3} \mathrm{~S}^{+}\left(\mathrm{M}+\mathrm{H}^{+}\right)$Expected 336.0512, Found 336.0501. 


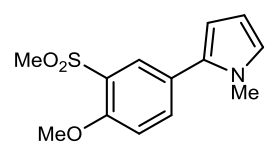

Prepared as described in General Procedure C: 2-mesylanisole (37 mg, $0.20 \mathrm{mmol}$ ), dibenzothiophene $S$-oxide (44 mg, $0.22 \mathrm{mmol}$ ), $\mathrm{Tf}_{2} \mathrm{O}$ ( $41 \mu \mathrm{L}, 0.24 \mathrm{mmol}$ ), PTH (5.5 mg, $\left.0.02 \mathrm{mmol}\right), \mathrm{N}$ methylpyrrole $(0.36 \mathrm{~mL}, 4.00 \mathrm{mmol}, 20$ equiv.), after purification by column chromatography on silica gel (50\% EtOAc in hexanes), gave $23(40.3 \mathrm{mg}, 0.15 \mathrm{mmol}, 76 \%)$ as a colourless oil; $\delta_{H}(400$ $\left.\mathrm{MHz}, \mathrm{CDCl}_{3}\right) 3.25\left(\mathrm{~s}, 3 \mathrm{H}, \mathrm{CH}_{3}\right), 3.64\left(\mathrm{~s}, 3 \mathrm{H}, \mathrm{NCH}_{3}\right), 4.03\left(\mathrm{~s}, 3 \mathrm{H}, \mathrm{OCH}_{3}\right), 6.19$ (dd, J = 3.6, $2.7 \mathrm{~Hz}, 1 \mathrm{H}, \mathrm{Ar}$ $H$ ), $6.22(\mathrm{dd}, J=3.6,1.8 \mathrm{~Hz}, 1 \mathrm{H}, \operatorname{Ar} H), 6.71(\mathrm{dd}, J=2.7,1.8 \mathrm{~Hz}, 1 \mathrm{H}, \operatorname{Ar} H), 7.10(\mathrm{~d}, J=8.6 \mathrm{~Hz}, 1 \mathrm{H}, \operatorname{Ar} H)$, $7.62(\mathrm{dd}, J=8.6,2.4 \mathrm{~Hz}, 1 \mathrm{H}, \operatorname{Ar} H), 8.01(\mathrm{~d}, J=2.4 \mathrm{~Hz}, 1 \mathrm{H}, \operatorname{Ar} H) ; \delta_{\mathrm{C}}\left(100 \mathrm{MHz}, \mathrm{CDCl}_{3}\right) 35.2\left(\mathrm{NCH}_{3}\right), 43.1$ $\left(\mathrm{CH}_{3}\right), 56.6\left(\mathrm{OCH}_{3}\right), 108.0(\mathrm{ArCH}), 109.2(\mathrm{ArCH}), 112.7(\mathrm{ArCH}), 124.2(\mathrm{ArCH}), 126.6(\operatorname{ArC}), 128.5(\mathrm{Ar}$ C), $129.5(\mathrm{ArCH}), 132.3(\operatorname{ArC}), 135.5(\mathrm{ArCH}), 156.0(\operatorname{ArC}) ; v_{\max }\left(\operatorname{thin}\right.$ film $\left./ \mathrm{cm}^{-1}\right)$ 719, 749, 831, 911, $958,1015,1064,1139,1281,1301,1429,1475,1492,1543,1568,2844,2942,3019$; MS (GCMS) m/z $265.0\left(\mathrm{M}^{+}\right) ; \mathrm{HRMS} \mathrm{C}_{13} \mathrm{H}_{16} \mathrm{O}_{3} \mathrm{NS}^{+}\left(\mathrm{M}+\mathrm{H}^{+}\right)$Expected 266.0845, Found 266.0845.

6-(1-Methyl-1H-pyrrol-2-yl)chroman-4-one, 24<smiles></smiles>

Prepared as described in General Procedure C: chroman-4-one (30 mg, $0.20 \mathrm{mmol}$ ), dibenzothiophene $S$-oxide (44 mg, $0.22 \mathrm{mmol}$ ), $\mathrm{Tf}_{2} \mathrm{O}$ ( $41 \mu \mathrm{L}, 0.24 \mathrm{mmol}$ ), PTH (5.5 mg, $\left.0.02 \mathrm{mmol}\right), \mathrm{N}$ methylpyrrole $(0.36 \mathrm{~mL}, 4.00 \mathrm{mmol}, 20$ equiv.), after purification by column chromatography on silica gel (20\% EtOAc in hexanes), gave $24(21.9 \mathrm{mg}, 0.10 \mathrm{mmol}, 48 \%)$ as a pale yellow oil; $\delta_{H}$ (400 $\left.\mathrm{MHz}, \mathrm{CDCl}_{3}\right) 2.84\left(\mathrm{t}, J=6.3 \mathrm{~Hz}, 2 \mathrm{H}, \mathrm{CH}_{2} \mathrm{C}(\mathrm{O})\right), 3.65\left(\mathrm{~s}, 3 \mathrm{H}, \mathrm{NCH}_{3}\right), 4.57\left(\mathrm{t}, J=6.3 \mathrm{~Hz}, 2 \mathrm{H}, \mathrm{CH}_{2} \mathrm{O}\right), 6.19$ (dd, J = 3.6, 2.7 Hz, 1H, Ar H), 6.21 (dd, J = 3.6, 1.9 Hz, 1H, Ar H), 6.70 (dd, J=2.7, 1.9 Hz, 1H, Ar H), $7.02(\mathrm{~d}, J=8.6 \mathrm{~Hz}, 1 \mathrm{H}, \operatorname{Ar} H), 7.53(\mathrm{dd}, J=8.6,2.4 \mathrm{~Hz}, 1 \mathrm{H}, \operatorname{Ar} H), 7.91(\mathrm{~d}, J=2.4 \mathrm{~Hz}, 1 \mathrm{H}, \operatorname{Ar} H) ; \delta_{\mathrm{c}}(100$ $\left.\mathrm{MHz}, \mathrm{CDCl}_{3}\right) 35.2\left(\mathrm{NCH}_{3}\right), 37.9\left(\mathrm{CH}_{2} \mathrm{C}(\mathrm{O})\right), 67.2\left(\mathrm{CH}_{2} \mathrm{O}\right), 107.9(\mathrm{ArCH}), 108.8(\mathrm{ArCH}), 118.2(\mathrm{ArCH})$, 121.2 (ArC), 123.8 (ArCH), 126.5 (ArCH), 127.0 (ArC), 133.2 (ArC), 136.5 (ArCH), $160.8(\operatorname{ArC}), 191.7$ $(C=0) ; v_{\max }\left(\right.$ thin film $\left./ \mathrm{cm}^{-1}\right) 713,831,872,914,927,1028,1063,1133,1172,1236,1279,1403,1461$, $1491,1615,1687,2878,2923,3054,3098 ; \mathrm{MS}(\mathrm{GCMS}) \mathrm{m} / \mathrm{z} 227.1\left(\mathrm{M}^{+}\right) ; \mathrm{HRMS} \mathrm{C}_{14} \mathrm{H}_{14} \mathrm{O}_{2} \mathrm{~N}^{+}\left(\mathrm{M}^{+} \mathrm{H}^{+}\right)$ Expected 228.1019, Found 228.1018. 
N,N-Diethyl-2-methoxy-5-(1-methyl-1H-pyrrol-2-yl)benzamide, 25<smiles></smiles>

Prepared as described in General Procedure C: $\mathbf{5 8}(42 \mathrm{mg}, 0.20 \mathrm{mmol}$ ), dibenzothiophene S-oxide (44 $\mathrm{mg}, 0.22 \mathrm{mmol}), \mathrm{Tf}_{2} \mathrm{O}(41 \mu \mathrm{L}, 0.24 \mathrm{mmol}), \mathrm{PTH}(5.5 \mathrm{mg}, 0.02 \mathrm{mmol}), \mathrm{N}$-methylpyrrole $(0.36 \mathrm{~mL}, 4.00$ mmol, 20 equiv.), after purification by column chromatography on silica gel (50\% EtOAc in hexanes), gave 25 (36.7 mg, $0.13 \mathrm{mmol}, 64 \%$ ) as a white solid; m.p. (recrystallized from $\mathrm{CHCl}_{3}$ ) $69-71{ }^{\circ} \mathrm{C} ; \delta_{\mathrm{H}}$ $\left(400 \mathrm{MHz}, \mathrm{CDCl}_{3}\right) 1.05\left(\mathrm{t}, J=7.1 \mathrm{~Hz}, 3 \mathrm{H}, \mathrm{CH}_{3}\right), 1.24\left(\mathrm{t}, J=7.1 \mathrm{~Hz}, 3 \mathrm{H}, \mathrm{CH}_{3}\right), 3.19\left(\mathrm{q}, J=7.1 \mathrm{~Hz}, 2 \mathrm{H}, \mathrm{CH}_{2}\right)$, $3.53-3.64\left(\mathrm{~m}, 2 \mathrm{H}, \mathrm{CH}_{2}\right), 3.63\left(\mathrm{~s}, 3 \mathrm{H}, \mathrm{NCH}_{3}\right), 3.85\left(\mathrm{~s}, 3 \mathrm{H}, \mathrm{OCH}_{3}\right), 6.13-6.21(\mathrm{~m}, 2 \mathrm{H}, \mathrm{Ar} \mathrm{H}), 6.65-6.71$ (m, 1H, Ar H), $6.94(\mathrm{~d}, J=8.6 \mathrm{~Hz}, 1 \mathrm{H}, \operatorname{Ar} H), 7.22$ (d, J = 2.3 Hz, 1H, Ar H), 7.34 (dd, J = 8.6, $2.3 \mathrm{~Hz}, 1 \mathrm{H}$, $\operatorname{Ar~H}) ; \delta_{\mathrm{C}}\left(100 \mathrm{MHz}, \mathrm{CDCl}_{3}\right) 13.0\left(\mathrm{CH}_{3}\right), 14.1\left(\mathrm{CH}_{3}\right), 35.1\left(\mathrm{NCH}_{3}\right), 38.9\left(\mathrm{CH}_{2}\right), 42.9\left(\mathrm{CH}_{2}\right), 55.8\left(\mathrm{OCH}_{3}\right)$, 107.8 ( $\mathrm{ArCH}), 108.4(\operatorname{ArCH}), 111.1(\operatorname{ArCH}), 123.4(\operatorname{ArCH}), 126.3(\operatorname{ArC}), 127.1(\operatorname{ArC}), 127.9(\operatorname{ArCH})$, 130.3 (ArCH), $133.8(\operatorname{ArC}), 154.4(\operatorname{Ar} C), 168.5(C=0) ; v_{\max }\left(\right.$ thin film $\left./ \mathrm{cm}^{-1}\right)$ 710, 781, 821, 877, 1022, $1085,1144,1175,1248,1292,1378,1440,1472,1543,1626,2837,2872,2970 ;$ MS (GCMS) m/z $286.1\left(\mathrm{M}^{+}\right)$; HRMS $\mathrm{C}_{17} \mathrm{H}_{23} \mathrm{O}_{2} \mathrm{~N}_{2}^{+}\left(\mathrm{M}+\mathrm{H}^{+}\right)$Expected 287.1754, Found 287.1748.

Methyl 2-methoxy-5-(1-methyl-1H-pyrrol-2-yl)benzoate, 26<smiles></smiles>

Prepared as described in General Procedure C: methyl 2-methoxybenzoate ( $29 \mu \mathrm{L}, 0.20 \mathrm{mmol}$ ), dibenzothiophene $S$-oxide (44 mg, $0.22 \mathrm{mmol}$ ), $\mathrm{Tf}_{2} \mathrm{O}$ (41 $\left.\mu \mathrm{L}, 0.24 \mathrm{mmol}\right)$, PTH ( $\left.5.5 \mathrm{mg}, 0.02 \mathrm{mmol}\right), \mathrm{N}$ methylpyrrole $(0.36 \mathrm{~mL}, 4.00 \mathrm{mmol}, 20$ equiv.), after purification by column chromatography on silica gel (20\% EtOAc in hexanes), gave 26 (32.9 mg, $0.13 \mathrm{mmol}, 67 \%$ ) as a white solid.

$10 \mathrm{mmol}$ scale reaction: methyl 2-methoxybenzoate $(1.44 \mathrm{~mL}, 10 \mathrm{mmol})$, dibenzothiophene $\mathrm{S}$-oxide (2.20 g, $11 \mathrm{mmol}), \mathrm{Tf}_{2} \mathrm{O}(2.02 \mathrm{~mL}, 12 \mathrm{mmol}), \mathrm{PTH}(275 \mathrm{mg}, 1.0 \mathrm{mmol}), \mathrm{N}$-methylpyrrole (17.8 mL, 200 mmol, 20 equiv.), after purification on silica gel (20\% EtOAc in hexanes), gave 26 (1.45 g, 5.9 mmol, $59 \%, 68 \%$ brsm) as a white solid; m.p. (recrystallized from $\left.\mathrm{CHCl}_{3}\right) 70-72{ }^{\circ} \mathrm{C} ; \delta_{\mathrm{H}}\left(400 \mathrm{MHz}, \mathrm{CDCl}_{3}\right)$ $3.63\left(\mathrm{~s}, 3 \mathrm{H}, \mathrm{NCH}_{3}\right), 3.90\left(\mathrm{~s}, 3 \mathrm{H}, \mathrm{OCH}_{3}\right), 3.94\left(\mathrm{~s}, 3 \mathrm{H}, \mathrm{OCH}_{3}\right), 6.15-6.22(\mathrm{~m}, 2 \mathrm{H}, \mathrm{Ar} \mathrm{H}), 6.67-6.73(\mathrm{~m}$, $1 \mathrm{H}, \operatorname{Ar} H), 7.02(\mathrm{~d}, J=8.6 \mathrm{~Hz}, 1 \mathrm{H}, \operatorname{Ar} H), 7.50(\mathrm{dd}, J=8.6,2.4 \mathrm{~Hz}, 1 \mathrm{H}, \operatorname{Ar} H), 7.84(\mathrm{~d}, J=2.4 \mathrm{~Hz}, 1 \mathrm{H}, \operatorname{Ar}$ H); $\delta_{\mathrm{C}}(100 \mathrm{MHz}, \mathrm{CDCl}) 35.0\left(\mathrm{NCH}_{3}\right), 52.2\left(\mathrm{OCH}_{3}\right), 56.3\left(\mathrm{OCH}_{3}\right), 107.8(\mathrm{ArCH}), 108.6(\mathrm{ArCH}), 112.2(\mathrm{Ar}$ $C H), 120.0(\operatorname{ArC}), 123.5(\operatorname{ArCH}), 125.7(\operatorname{ArC}), 132.0(\operatorname{ArCH}), 133.3(\operatorname{ArC}), 133.8(\operatorname{ArCH}), 158.1(\operatorname{Ar} C)$, 
$166.6(C=0) ; v_{\max }\left(\right.$ thin film/cm $\left.{ }^{-1}\right) 712,784,821,965,1021,1081,1229,1255,1315,1463,1497$, $1543,1574,1699,1726,2838,2949,3097$; MS (GCMS) $m / z 245.1\left(\mathrm{M}^{+}\right) ; \mathrm{HRMS} \mathrm{C}_{14} \mathrm{H}_{16} \mathrm{O}_{3} \mathrm{~N}^{+}\left(\mathrm{M}^{+} \mathrm{H}^{+}\right)$ Expected 246.1125, Found 246.1120.

2-(2-Methoxy-5-(trifluoromethyl)phenyl)-1-methyl-1H-pyrrole, 27<smiles></smiles>

Prepared as described in General Procedure C: 4-trifluoromethylanisole ( $29 \mu \mathrm{L}, 0.20 \mathrm{mmol})$, dibenzothiophene S-oxide (44 mg, $0.22 \mathrm{mmol}$ ), $\mathrm{Tf}_{2} \mathrm{O}$ (41 $\mu \mathrm{L}, 0.24 \mathrm{mmol}$ ), PTH (5.5 mg, $\left.0.02 \mathrm{mmol}\right), \mathrm{N}$ methylpyrrole $(0.36 \mathrm{~mL}, 4.00 \mathrm{mmol}, 20$ equiv.), after purification by column chromatography on silica gel (30\% $\mathrm{CHCl}_{3}$ in hexanes), gave $27(41.6 \mathrm{mg}, 0.16 \mathrm{mmol}, 82 \%)$ as a white solid; m.p. (recrystallized from $\left.\mathrm{CHCl}_{3}\right) 67-69^{\circ} \mathrm{C} ; \delta_{\mathrm{H}}\left(400 \mathrm{MHz}, \mathrm{CDCl}_{3}\right) 3.50\left(\mathrm{~s}, 3 \mathrm{H}, \mathrm{NCH}_{3}\right), 3.88\left(\mathrm{~s}, 3 \mathrm{H}, \mathrm{OCH}_{3}\right), 6.20$ (dd, J = 3.6, 1.8 Hz, 1H, Ar H), $6.24(\mathrm{dd}, J=3.6,2.7 \mathrm{~Hz}, 1 \mathrm{H}, \operatorname{Ar} H), 6.73-6.79(\mathrm{~m}, 1 \mathrm{H}, \operatorname{Ar} H), 7.03$ (d, J = 8.6 Hz, 1H, Ar H), $7.56(\mathrm{~d}, J=2.4 \mathrm{~Hz}, 1 \mathrm{H}, \operatorname{Ar} H), 7.57-7.65(\mathrm{~m}, 1 \mathrm{H}, \operatorname{Ar} H) ; \delta_{\mathrm{C}}\left(100 \mathrm{MHz}, \mathrm{CDCl}_{3}\right) 34.7$ $\left(\mathrm{NCH}_{3}\right), 55.9\left(\mathrm{OCH}_{3}\right), 107.9(\mathrm{ArCH}), 109.8(\mathrm{ArCH}), 110.7(\mathrm{ArCH}), 123.0(\mathrm{q}, J=32.8 \mathrm{~Hz}, \mathrm{ArC}), 123.2(\mathrm{Ar}$ C), 123.3 ( $\mathrm{ArCH}$ ), 124.2 (q, J = 271.0 Hz, CF $), 126.5$ (q, J = 3.8 Hz, $\mathrm{ArCH}$ ), $129.5(q, J=3.6 \mathrm{~Hz}, \mathrm{ArCH}$ ), $129.7(\operatorname{ArC}), 159.8(\operatorname{ArC}) ; \delta_{\mathrm{F}}\left(376 \mathrm{MHz}, \mathrm{CDCl}_{3}\right)-61.5 ; v_{\max }\left(\right.$ thin film/ $\left./ \mathrm{cm}^{-1}\right)$ 711, 818, 907, 1022, 1045,

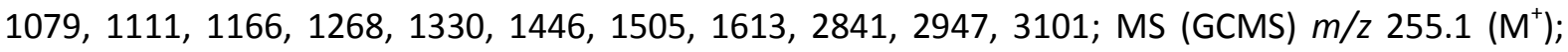
HRMS $\mathrm{C}_{13} \mathrm{H}_{13} \mathrm{ONF}_{3}{ }^{+}\left(\mathrm{M}+\mathrm{H}^{+}\right)$Expected 256.0944, Found 256.0939.

4-Methoxy-3-(1-methyl-1H-pyrrol-2-yl)benzonitrile, 28<smiles></smiles>

Prepared as described in General Procedure C: 4-methoxybenzonitrile (26 mg, $0.20 \mathrm{mmol}$ ), dibenzothiophene $S$-oxide ( $44 \mathrm{mg}, 0.22 \mathrm{mmol}$ ), $\mathrm{Tf}_{2} \mathrm{O}(41 \mu \mathrm{L}, 0.24 \mathrm{mmol}$ ), PTH (5.5 mg, $0.02 \mathrm{mmol}$ ), $\mathrm{N}$ methylpyrrole ( $0.36 \mathrm{~mL}, 4.00 \mathrm{mmol}, 20$ equiv.), after purification by column chromatography on silica gel (20\% EtOAc in hexanes), gave $28(30.1 \mathrm{mg}, 0.14 \mathrm{mmol}, 71 \%)$ as a colourless oil; $\delta_{\mathrm{H}}(400$ $\left.\mathrm{MHz}, \mathrm{CDCl}_{3}\right) 3.48\left(\mathrm{~s}, 3 \mathrm{H}, \mathrm{NCH}_{3}\right), 3.89\left(\mathrm{~s}, 3 \mathrm{H}, \mathrm{OCH}_{3}\right), 6.14-6.20(\mathrm{~m}, 1 \mathrm{H}, \mathrm{Ar} \mathrm{H}), 6.19-6.26(\mathrm{~m}, 1 \mathrm{H}, \mathrm{Ar}$ H), $6.72-6.79(\mathrm{~m}, 1 \mathrm{H}, \operatorname{Ar} H), 7.01(\mathrm{~d}, J=8.6 \mathrm{~Hz}, 1 \mathrm{H}, \operatorname{Ar} H), 7.56(\mathrm{~d}, J=2.1 \mathrm{~Hz}, 1 \mathrm{H}, \operatorname{Ar} H), 7.65(\mathrm{dd}, J=$ 8.6, 2.1 Hz, 1H, $\mathrm{Ar} \mathrm{H})$; $\delta_{\mathrm{C}}\left(100 \mathrm{MHz}, \mathrm{CDCl}_{3}\right) 34.8\left(\mathrm{NCH}_{3}\right), 56.0\left(\mathrm{OCH}_{3}\right), 104.2(\operatorname{ArC}), 108.1(\operatorname{ArCH}), 110.2$ $(\operatorname{ArCH}), 111.4(\operatorname{ArCH}), 119.1(C \equiv N), 123.6(\operatorname{ArCH}), 124.1(\operatorname{ArC}), 128.7(\operatorname{ArC}), 133.7(\operatorname{ArCH}), 135.9(\operatorname{Ar}$ 
$C H), 160.7(\operatorname{ArC}) ; v_{\max }\left(\operatorname{thin}\right.$ film/cm $\mathrm{cm}^{-1}$ ) 714, 817, 892, 904, 1018, 1056, 1142, 1259, 1295, 1463, 1495, 1540, 1580, 1600, 2222, 2841, 2943, 3099; MS (GCMS) m/z $212.1\left(\mathrm{M}^{+}\right) ; \mathrm{HRMS} \mathrm{C}_{13} \mathrm{H}_{13} \mathrm{ON}_{2}{ }^{+}\left(\mathrm{M}^{+} \mathrm{H}^{+}\right)$ Expected 213.1022, Found 213.1019.

Methyl 2-methoxy-5-(1H-pyrrol-2-yl)benzoate, 29<smiles>COc1ccc(-c2ccc[nH]2)cc1C(=O)O</smiles>

Prepared as described in General Procedure C: methyl 2-methoxybenzoate ( $29 \mu \mathrm{L}, 0.20 \mathrm{mmol})$, dibenzothiophene $S$-oxide (44 mg, $0.22 \mathrm{mmol}$ ), $\mathrm{Tf}_{2} \mathrm{O}(41 \mu \mathrm{L}, 0.24 \mathrm{mmol}$ ), PTH (5.5 mg, $0.02 \mathrm{mmol}$ ), $N$ H-pyrrole $(0.28 \mathrm{~mL}, 4.00 \mathrm{mmol}, 20$ equiv.), after purification by column chromatography on silica gel (30\% EtOAc in hexanes), gave $29(23.9 \mathrm{mg}, 0.10 \mathrm{mmol}, 52 \%)$ as a white solid; m.p. (recrystallized from $\left.\mathrm{CHCl}_{3}\right) 93-95^{\circ} \mathrm{C} ; \delta_{\mathrm{H}}\left(400 \mathrm{MHz}, \mathrm{CDCl}_{3}\right) 3.89\left(\mathrm{~s}, 3 \mathrm{H}, \mathrm{OCH}_{3}\right), 3.91\left(\mathrm{~s}, 3 \mathrm{H}, \mathrm{OCH}_{3}\right), 6.29$ (dd, J = 3.9, $2.7 \mathrm{~Hz}, 1 \mathrm{H}, \operatorname{Ar} H), 6.45$ (dd, J = 3.9, $1.5 \mathrm{~Hz}, 1 \mathrm{H}, \operatorname{Ar} H), 6.84$ (dd, J = 2.7, $1.5 \mathrm{~Hz}, 1 \mathrm{H}, \operatorname{Ar} H), 6.96$ (d, J = 8.7 $\mathrm{Hz}, 1 \mathrm{H}, \operatorname{Ar} H), 7.58(\mathrm{dd}, J=8.7,2.5 \mathrm{~Hz}, 1 \mathrm{H}, \operatorname{Ar} H), 7.90(\mathrm{~d}, J=2.5 \mathrm{~Hz}, 1 \mathrm{H}, \operatorname{Ar} H), 8.56(\mathrm{~s}, 1 \mathrm{H}, \mathrm{NH}) ; \delta_{\mathrm{C}}$ $\left(100 \mathrm{MHz}, \mathrm{CDCl}_{3}\right) 52.3\left(\mathrm{OCH}_{3}\right), 56.3\left(\mathrm{OCH}_{3}\right), 105.6(\mathrm{ArCH}), 110.1(\mathrm{ArCH}), 112.8(\mathrm{ArCH}), 118.8(\mathrm{ArCH})$, 120.3 ( $\operatorname{Ar} C), 125.7(\operatorname{Ar} C), 127.2(\operatorname{Ar} C H), 129.3(\operatorname{Ar} C H), 131.1(\operatorname{Ar} C), 157.7(\operatorname{Ar} C), 166.8(C=0) ; v_{\max }$ (thin film/ $\mathrm{cm}^{-1}$ ) 722, 787, 820, 1020, 1060, 1085, 1182, 1234, 1255, 1315, 1434, 1462, 1498, 1564, $1578,1709,2839,2948,3001,3098,3356$; MS (GCMS) $m / z 231.1\left(\mathrm{M}^{+}\right) ; \mathrm{HRMS} \mathrm{C}_{13} \mathrm{H}_{14} \mathrm{O}_{3} \mathrm{~N}^{+}\left(\mathrm{M}^{+} \mathrm{H}^{+}\right)$ Expected 232.0968, Found 232.0965.

Methyl 2-methoxy-5-(1-phenyl-1H-pyrrol-2-yl)benzoate, 30<smiles>COC(=O)c1cc(-c2cccn2-c2ccccc2)ccc1O</smiles>

Prepared as described in General Procedure C: methyl 2-methoxybenzoate ( $29 \mu \mathrm{L}, 0.20 \mathrm{mmol}$ ), dibenzothiophene S-oxide (44 mg, $0.22 \mathrm{mmol}$ ), $\mathrm{Tf}_{2} \mathrm{O}$ (41 $\left.\mu \mathrm{L}, 0.24 \mathrm{mmol}\right), \mathrm{PTH}(5.5 \mathrm{mg}, 0.02 \mathrm{mmol}), \mathrm{N}$ phenylpyrrole ( $286 \mathrm{mg}, 2.00 \mathrm{mmol}, 10$ equiv.), after purification by column chromatography on silica gel (20\% EtOAc in hexanes), gave $30(41.8 \mathrm{mg}, 0.14 \mathrm{mmol}, 68 \%)$ as a colourless oil; $\delta_{\mathrm{H}}(400 \mathrm{MHz}$, $\left.\mathrm{CDCl}_{3}\right) 3.83\left(\mathrm{~s}, 3 \mathrm{H}, \mathrm{OCH}_{3}\right), 3.86\left(\mathrm{~s}, 3 \mathrm{H}, \mathrm{OCH}_{3}\right), 6.35(\mathrm{dd}, J=3.6,2.8 \mathrm{~Hz}, 1 \mathrm{H}, \mathrm{Ar} \mathrm{H}), 6.41(\mathrm{dd}, J=3.6,1.8$ $\mathrm{Hz}, 1 \mathrm{H}, \operatorname{Ar} H), 6.78(\mathrm{~d}, J=8.7 \mathrm{~Hz}, 1 \mathrm{H}, \operatorname{Ar} H), 6.93(\mathrm{dd}, J=2.8,1.8 \mathrm{~Hz}, 1 \mathrm{H}, \operatorname{Ar} H), 7.09$ (dd, J = 8.7, 2.4 Hz, $1 \mathrm{H}, \operatorname{Ar} H), 7.11-7.20(\mathrm{~m}, 2 \mathrm{H}, \operatorname{Ar} H), 7.23-7.33(\mathrm{~m}, 1 \mathrm{H}, \operatorname{Ar} H), 7.29-7.40(\mathrm{~m}, 2 \mathrm{H}, \operatorname{Ar} H), 7.69(\mathrm{~d}, J=$ 
2.4 Hz, $1 \mathrm{H}, \operatorname{Ar~H}) ; \delta_{\mathrm{C}}\left(100 \mathrm{MHz}, \mathrm{CDCl}_{3}\right) 52.1\left(\mathrm{OCH}_{3}\right), 56.2\left(\mathrm{OCH}_{3}\right), 109.3(\mathrm{ArCH}), 110.4(\mathrm{ArCH}), 111.8$ ( $\operatorname{ArCH}), 119.9(\operatorname{ArC}), 124.2(\operatorname{ArCH}), 125.5(\operatorname{ArC}), 125.9(\operatorname{Ar} C H), 126.8(\operatorname{ArCH}), 129.2(\operatorname{ArCH}), 131.7$ $(\operatorname{Ar} C H), 132.6(\operatorname{ArC}), 133.3(\operatorname{Ar} C H), 140.4(\operatorname{ArC}), 157.7(\operatorname{ArC}), 166.6(C=0) ; v_{\max }\left(\operatorname{thin}\right.$ film/ $\left.\mathrm{cm}^{-1}\right) 764$, $822,950,1022,1070,1084,1154,1185,1232,1263,1301,1435,1454,1497,1544,1597,1728$, 2838, 2947, 3002, 3102; MS (GCMS) $m / z 307.1\left(\mathrm{M}^{+}\right)$; HRMS $\mathrm{C}_{19} \mathrm{H}_{18} \mathrm{O}_{3} \mathrm{~N}^{+}\left(\mathrm{M}+\mathrm{H}^{+}\right)$Expected 308.1281, Found 308.1276 .

tert-Butyl 2-(4-methoxy-3-(methoxycarbonyl)phenyl)-1H-pyrrole-1-carboxylate, 31<smiles>COc1ccc(-c2cccn2C(=O)O)cc1C(=O)O</smiles>

Prepared as described in General Procedure C: methyl 2-methoxybenzoate ( $29 \mu \mathrm{L}, 0.20 \mathrm{mmol}$ ), dibenzothiophene $S$-oxide ( $44 \mathrm{mg}, 0.22 \mathrm{mmol}$ ), $\mathrm{Tf}_{2} \mathrm{O}(41 \mu \mathrm{L}, 0.24 \mathrm{mmol}$ ), PTH (5.5 mg, $0.02 \mathrm{mmol}), \mathrm{N}$ Boc-pyrrole ( $0.33 \mathrm{~mL}, 2.00 \mathrm{mmol}, 10$ equiv.), after purification by column chromatography on silica gel (20\% EtOAc in hexanes), gave $31(50.6 \mathrm{mg}, 0.15 \mathrm{mmol}, 76 \%)$ as a colourless oil; $\delta_{\mathrm{H}}(400 \mathrm{MHz}$, $\left.\mathrm{CDCl}_{3}\right) 1.40\left(\mathrm{~s}, 9 \mathrm{H}, \mathrm{CH}_{3}\right), 3.90\left(\mathrm{~s}, 3 \mathrm{H}, \mathrm{OCH}_{3}\right), 3.95\left(\mathrm{~s}, 3 \mathrm{H}, \mathrm{OCH}_{3}\right), 6.14-6.21(\mathrm{~m}, 1 \mathrm{H}, \mathrm{Ar} \mathrm{H}), 6.20-6.27$ (m, 1H, Ar H), 6.99 (d, J = 8.6 Hz, 1H, Ar H), $7.35-7.40$ (m, 1H, Ar H), 7.49 (dd, J = 8.6, 2.4 Hz, 1H, Ar $H), 7.83(\mathrm{~d}, J=2.3 \mathrm{~Hz}, 1 \mathrm{H}, \operatorname{Ar} H) ; \delta_{\mathrm{C}}\left(100 \mathrm{MHz}, \mathrm{CDCl}_{3}\right) 27.8\left(\mathrm{CH}_{3}\right), 52.1\left(\mathrm{OCH}_{3}\right), 56.2\left(\mathrm{OCH}_{3}\right), 83.9$ $\left(\mathrm{CH}_{3} \mathrm{C}\right), 110.7(\mathrm{ArCH}), 111.3(\mathrm{ArCH}), 114.6(\mathrm{ArCH}), 119.1(\operatorname{ArC}), 122.7(\operatorname{ArCH}), 126.6(\operatorname{ArC}), 132.9(\operatorname{Ar}$ $C H), 133.6(\operatorname{ArC}), 134.3(\operatorname{Ar} C H), 149.4(\operatorname{Ar} C), 158.6(C=0), 166.5(C=0) ; v_{\max }\left(\right.$ thin film $\left./ \mathrm{cm}^{-1}\right) 730,771$, $813,832,962,1022,1080,1142,1253,1311,1504,1579,1728,2839,2950,2978$; MS (GCMS) $m / z$ $331.1\left(\mathrm{M}^{+}\right)$; HRMS $\mathrm{C}_{18} \mathrm{H}_{22} \mathrm{O}_{5} \mathrm{~N}^{+}\left(\mathrm{M}+\mathrm{H}^{+}\right)$Expected 332.1492, Found 332.1484.

Methyl 5-(4-methoxy-3-(methoxycarbonyl)phenyl)-1-methyl-1H-pyrrole-2-carboxylate, 32<smiles></smiles>

Prepared as described in General Procedure C: methyl 2-methoxybenzoate ( $29 \mu \mathrm{L}, 0.20 \mathrm{mmol}$ ), dibenzothiophene $S$-oxide ( $44 \mathrm{mg}, 0.22 \mathrm{mmol}$ ), $\mathrm{Tf}_{2} \mathrm{O}$ ( $41 \mu \mathrm{L}, 0.24 \mathrm{mmol}$ ), PTH (5.5 mg, $0.02 \mathrm{mmol}$ ), methyl 2-pyrrolecarboxylate $(0.52 \mathrm{~mL}, 4.00 \mathrm{mmol}, 20$ equiv.), after purification by column chromatography on silica gel (20\% EtOAc in hexanes), gave 32 (36.9 mg, $0.12 \mathrm{mmol}, 61 \%$ ) as a white solid; m.p. (recrystallized from $\left.\mathrm{CHCl}_{3}\right) 104-106{ }^{\circ} \mathrm{C} ; \delta_{\mathrm{H}}\left(400 \mathrm{MHz}, \mathrm{CDCl}_{3}\right) 3.83\left(\mathrm{~s}, 3 \mathrm{H}, \mathrm{OCH}_{3}\right), 3.85$ (s, $\left.3 \mathrm{H}, \mathrm{NCH}_{3}\right), 3.90\left(\mathrm{~s}, 3 \mathrm{H}, \mathrm{OCH}_{3}\right), 3.95\left(\mathrm{~s}, 3 \mathrm{H}, \mathrm{OCH}_{3}\right), 6.17(\mathrm{~d}, J=4.0 \mathrm{~Hz}, 1 \mathrm{H}, \mathrm{Ar} H), 7.00(\mathrm{~d}, J=4.0 \mathrm{~Hz}, 1 \mathrm{H}$, 
$\operatorname{Ar} H), 7.05(\mathrm{~d}, J=8.6 \mathrm{~Hz}, 1 \mathrm{H}, \operatorname{Ar} H), 7.48(\mathrm{dd}, J=8.6,2.4 \mathrm{~Hz}, 1 \mathrm{H}, \operatorname{Ar} H), 7.83(\mathrm{~d}, J=2.4 \mathrm{~Hz}, 1 \mathrm{H}, \operatorname{Ar} H) ; \delta_{\mathrm{C}}$ $\left(100 \mathrm{MHz}, \mathrm{CDCl}_{3}\right) 34.4\left(\mathrm{NCH}_{3}\right), 51.2\left(\mathrm{OCH}_{3}\right), 52.3\left(\mathrm{OCH}_{3}\right), 56.3\left(\mathrm{OCH}_{3}\right), 109.2(\mathrm{ArCH}), 112.3(\mathrm{ArCH})$, 117.8 (ArCH), $120.3(\operatorname{ArC}), 123.4(\operatorname{ArC}), 124.4(\operatorname{ArC}), 132.8(\operatorname{ArCH}), 134.4(\operatorname{ArCH}), 140.4(\operatorname{ArC} C), 159.0$ $(\operatorname{Ar} C), 162.0(C=0), 166.3(C=0) ; v_{\max }\left(\right.$ thin film $\left./ \mathrm{cm}^{-1}\right)$ 753, 785, 822, 914, 1021, 1082, 1106, 1183, 1238, 1300, 1433, 1454, 1539, 1575, 1697, 1729, 2841, 2949, 2997; MS (GCMS) m/z $303.1\left(\mathrm{M}^{+}\right)$; HRMS $\mathrm{C}_{16} \mathrm{H}_{18} \mathrm{O}_{5} \mathrm{~N}^{+}\left(\mathrm{M}+\mathrm{H}^{+}\right)$Expected 304.1179, Found 304.1176.

Methyl 2-methoxy-5-(thiophen-2-yl)benzoate, 33<smiles>COc1ccc(-c2cccs2)cc1C(=O)O</smiles>

Prepared as described in General Procedure C: methyl 2-methoxybenzoate ( $29 \mu \mathrm{L}, 0.20 \mathrm{mmol}$ ), dibenzothiophene $S$-oxide (44 mg, $0.22 \mathrm{mmol}$ ), $\mathrm{Tf}_{2} \mathrm{O}(41 \mu \mathrm{L}, 0.24 \mathrm{mmol}), \mathrm{PTH}(5.5 \mathrm{mg}, 0.02 \mathrm{mmol}$ ), thiophene ( $0.32 \mathrm{~mL}, 4.00 \mathrm{mmol}, 20$ equiv.), after purification by column chromatography on silica gel (20\% EtOAc in hexanes), gave $33(29.3 \mathrm{mg}, 0.12 \mathrm{mmol}, 59 \%)$ as a white solid; m.p. (recrystallized from $\left.\mathrm{CHCl}_{3}\right) 85-87^{\circ} \mathrm{C} ; \delta_{\mathrm{H}}\left(400 \mathrm{MHz}, \mathrm{CDCl}_{3}\right) 3.94\left(\mathrm{~s}, 3 \mathrm{H}, \mathrm{OCH}_{3}\right), 3.95\left(\mathrm{~s}, 3 \mathrm{H}, \mathrm{OCH}_{3}\right), 7.01(\mathrm{~d}, J=8.7 \mathrm{~Hz}$, $1 \mathrm{H}, \operatorname{Ar} H), 7.08(\mathrm{dd}, J=4.9,3.9 \mathrm{~Hz}, 1 \mathrm{H}, \operatorname{Ar} H), 7.24-7.30(\mathrm{~m}, 2 \mathrm{H}, \operatorname{Ar} H), 7.71(\mathrm{dd}, J=8.7,2.5 \mathrm{~Hz}, 1 \mathrm{H}, \operatorname{Ar}$ $H), 8.05(\mathrm{~d}, J=2.5 \mathrm{~Hz}, 1 \mathrm{H}, \operatorname{Ar} H) ; \delta_{\mathrm{c}}\left(100 \mathrm{MHz}, \mathrm{CDCl}_{3}\right) 52.3\left(\mathrm{OCH}_{3}\right), 56.3\left(\mathrm{OCH}_{3}\right), 112.6(\operatorname{ArCH}), 120.5$ $(\operatorname{ArC}), 122.9(\operatorname{ArCH}), 124.6(\operatorname{ArCH}), 127.1(\operatorname{ArC}), 128.2(\operatorname{ArCH}), 129.3(\operatorname{ArCH}), 131.0(\operatorname{ArCH}), 143.2$ $(\operatorname{ArC}), 158.6(\operatorname{Ar} C), 166.5(C=0) ; v_{\max }\left(\right.$ thin film $/ \mathrm{cm}^{-1}$ ) 814, 858, 956, 1008, 1025, 1081, 1207, 1234, $1263,1306,1432,1493,1574,1610,1727,2837,2947,3002$; MS (GCMS) m/z $248.0\left(\mathrm{M}^{+}\right)$; HRMS $\mathrm{C}_{13} \mathrm{H}_{12} \mathrm{O}_{3} \mathrm{~S}^{+}\left(\mathrm{M}^{+}\right)$Expected 248.0502, Found 248.0510.

Methyl 5-(3,4-dibromothiophen-2-yl)-2-methoxybenzoate, 34<smiles>COc1ccc(-c2scc(Br)c2Br)cc1C(=O)O[Na]</smiles>

Prepared as described in General Procedure C: methyl 2-methoxybenzoate ( $29 \mu \mathrm{L}, 0.20 \mathrm{mmol}$ ), dibenzothiophene $S$-oxide (44 mg, $0.22 \mathrm{mmol}$ ), $\mathrm{Tf}_{2} \mathrm{O}$ ( $41 \mu \mathrm{L}, 0.24 \mathrm{mmol}$ ), PTH (5.5 mg, $0.02 \mathrm{mmol}$ ), 3,4-dibromothiophene $(0.44 \mathrm{~mL}, 4.00 \mathrm{mmol}, 20$ equiv.), after purification by column chromatography on silica gel (15\% EtOAc in hexanes), gave $34(50.4 \mathrm{mg}, 0.12 \mathrm{mmol}, 62 \%)$ as a white solid; m.p. (recrystallized from $\left.\mathrm{CHCl}_{3}\right) 126-128{ }^{\circ} \mathrm{C} ; \delta_{\mathrm{H}}\left(400 \mathrm{MHz}, \mathrm{CDCl}_{3}\right) 3.91\left(\mathrm{~s}, 3 \mathrm{H}, \mathrm{OCH}_{3}\right), 3.95(\mathrm{~s}$, 
$\left.3 \mathrm{H}, \mathrm{OCH}_{3}\right), 7.05(\mathrm{~d}, J=8.7 \mathrm{~Hz}, 1 \mathrm{H}, \operatorname{Ar} H), 7.35(\mathrm{~s}, 1 \mathrm{H}, \operatorname{Ar} H), 7.72(\mathrm{dd}, J=8.7,2.5 \mathrm{~Hz}, 1 \mathrm{H}, \operatorname{Ar} H), 8.02(\mathrm{~d}$, $J=2.5 \mathrm{~Hz}, 1 \mathrm{H}, \operatorname{Ar} H) ; \delta_{\mathrm{C}}\left(100 \mathrm{MHz}, \mathrm{CDCl}_{3}\right) 52.4\left(\mathrm{OCH}_{3}\right), 56.3\left(\mathrm{OCH}_{3}\right), 111.6(\operatorname{ArC}), 112.3(\operatorname{ArCH}), 114.8$ $(\operatorname{ArC}), 120.4(\operatorname{ArC}), 122.2(\operatorname{ArCH}), 125.1(\operatorname{ArC}), 132.5(\operatorname{ArCH}), 134.1(\operatorname{ArCH}), 138.3(\operatorname{ArC}), 159.5(\operatorname{Ar}$ C), $166.1(C=0) ; v_{\max }\left(\right.$ thin film/cm $\mathrm{cm}^{-1}$ ) 751, 824, 839, 881, 967, 1008, 1014, 1026, 1120, 1157, 1262, $1313,1398,1433,1485,1521,1609,1710,1734,2839,2942,3003,3132$; MS (GCMS) m/z 403.2 $\left(\mathrm{M}^{+}\right) ; \mathrm{HRMS} \mathrm{C}_{13} \mathrm{H}_{11} \mathrm{O}_{3} \mathrm{Br}_{2} \mathrm{~S}^{+}\left(\mathrm{M}+\mathrm{H}^{+}\right)$Expected 404.8790, Found 404.8793.

Methyl 5-(4-acetylthiophen-2-yl)-2-methoxybenzoate, 35<smiles>COc1ccc(-c2cc(C(C)=O)cs2)cc1C(=O)O[Na]</smiles>

Prepared as described in General Procedure C: methyl 2-methoxybenzoate ( $29 \mu \mathrm{L}, 0.20 \mathrm{mmol}$ ), dibenzothiophene S-oxide (44 mg, $0.22 \mathrm{mmol}$ ), $\mathrm{Tf}_{2} \mathrm{O}$ (41 $\left.\mu \mathrm{L}, 0.24 \mathrm{mmol}\right), \mathrm{PTH}(5.5 \mathrm{mg}, 0.02 \mathrm{mmol}$ ), 3acetylthiophene ( $505 \mathrm{mg}, 4.00 \mathrm{mmol}, 20$ equiv.), after purification by column chromatography on silica gel (30\% EtOAc in hexanes), gave $35(37.6 \mathrm{mg}, 0.13 \mathrm{mmol}, 65 \%)$ as a white solid; m.p. (recrystallized from $\left.\mathrm{CHCl}_{3}\right) 86-88^{\circ} \mathrm{C} ; \delta_{\mathrm{H}}\left(400 \mathrm{MHz}, \mathrm{CDCl}_{3}\right) 2.26\left(\mathrm{~s}, 3 \mathrm{H}, \mathrm{CH}_{3}\right), 3.89\left(\mathrm{~s}, 3 \mathrm{H}, \mathrm{OCH}_{3}\right), 3.95$ $\left(\mathrm{s}, 3 \mathrm{H}, \mathrm{OCH}_{3}\right), 7.03(\mathrm{~d}, J=8.6 \mathrm{~Hz}, 1 \mathrm{H}, \operatorname{Ar} H), 7.23(\mathrm{~d}, J=5.3 \mathrm{~Hz}, 1 \mathrm{H}, \operatorname{Ar} H), 7.43(\mathrm{~d}, J=5.3 \mathrm{~Hz}, 1 \mathrm{H}, \operatorname{Ar} H)$, $7.58(\mathrm{dd}, J=8.6,2.4 \mathrm{~Hz}, 1 \mathrm{H}, \operatorname{Ar} H), 7.90(\mathrm{~d}, J=2.4 \mathrm{~Hz}, 1 \mathrm{H}, \operatorname{Ar} H) ; \delta_{\mathrm{C}}\left(100 \mathrm{MHz}, \mathrm{CDCl}_{3}\right) 30.1\left(\mathrm{CH}_{3}\right), 52.3$ $\left(\mathrm{OCH}_{3}\right), 56.3\left(\mathrm{OCH}_{3}\right), 112.1(\mathrm{ArCH}), 120.0(\mathrm{ArC}), 124.3(\mathrm{ArCH}), 125.5(\operatorname{ArC}), 129.4(\operatorname{ArCH}), 133.2(\mathrm{Ar}$ $\mathrm{CH}), 135.0(\mathrm{ArCH}), 137.9(\operatorname{ArC}), 148.4(\operatorname{ArC}), 159.7(\operatorname{ArC}), 166.1(C=0), 194.5(C=0) ; v_{\max }$ (thin film/cm ${ }^{-1}$ ) 737, 817, 824, 878, 959, 1011, 1024, 1081, 1139, 1182, 1246, 1270, 1305, 1431, 1493, $1572,1608,1673,1727,2840,2948,3001,3107$; MS (GCMS) m/z $290.1\left(\mathrm{M}^{+}\right)$; HRMS $\mathrm{C}_{15} \mathrm{H}_{15} \mathrm{O}_{4} \mathrm{~S}^{+}$ $\left(\mathrm{M}+\mathrm{H}^{+}\right)$Expected 291.0686, Found 291.0682.

Methyl 5-(furan-2-yl)-2-methoxybenzoate, 36<smiles>COc1ccc(-c2ccco2)cc1C(N)=O</smiles>

Prepared as described in General Procedure C: methyl 2-methoxybenzoate ( $29 \mu \mathrm{L}, 0.20 \mathrm{mmol}$ ), dibenzothiophene $S$-oxide (44 mg, $0.22 \mathrm{mmol}$ ), $\mathrm{Tf}_{2} \mathrm{O}$ (41 $\mu \mathrm{L}, 0.24 \mathrm{mmol}$ ), PTH (5.5 mg, $0.02 \mathrm{mmol}$ ), furan $(0.29 \mathrm{~mL}, 4.00 \mathrm{mmol}, 20$ equiv.), after purification by column chromatography on silica gel 
(20\% EtOAc in hexanes), gave $36(26.4 \mathrm{mg}, 0.11 \mathrm{mmol}, 57 \%)$ as a white solid; m.p. (recrystallized from $\left.\mathrm{CHCl}_{3}\right) 75-77^{\circ} \mathrm{C} ; \delta_{\mathrm{H}}\left(400 \mathrm{MHz}, \mathrm{CDCl}_{3}\right) 3.92\left(\mathrm{~s}, 3 \mathrm{H}, \mathrm{OCH}_{3}\right), 3.93\left(\mathrm{~s}, 3 \mathrm{H}, \mathrm{OCH}_{3}\right), 6.46(\mathrm{dd}, J=3.3$, $1.8 \mathrm{~Hz}, 1 \mathrm{H}, \operatorname{Ar} H), 6.58(\mathrm{dd}, J=3.3,0.8 \mathrm{~Hz}, 1 \mathrm{H}, \operatorname{Ar} H), 7.00(\mathrm{~d}, J=8.7 \mathrm{~Hz}, 1 \mathrm{H}, \operatorname{Ar} H), 7.44(\mathrm{dd}, J=1.8,0.8$ $\mathrm{Hz}, 1 \mathrm{H}, \operatorname{Ar} H), 7.76(\mathrm{dd}, J=8.7,2.4 \mathrm{~Hz}, 1 \mathrm{H}, \operatorname{Ar} H), 8.09(\mathrm{~d}, J=2.4 \mathrm{~Hz}, 1 \mathrm{H}, \operatorname{Ar} H) ; \delta_{\mathrm{C}}\left(100 \mathrm{MHz}, \mathrm{CDCl}_{3}\right)$ $52.3\left(\mathrm{OCH}_{3}\right), 56.3\left(\mathrm{OCH}_{3}\right), 104.4(\operatorname{ArCH}), 111.8(\mathrm{ArCH}), 112.5(\operatorname{ArCH}), 120.4(\operatorname{ArC}), 123.8(\operatorname{ArC}), 127.4$ $(\operatorname{ArCH}), 128.9(\mathrm{ArCH}), 141.9(\operatorname{ArCH}), 153.0(\operatorname{ArC}), 158.5(\operatorname{ArC}), 166.5(C=0) ; v_{\max }\left(\right.$ thin film $\left./ \mathrm{cm}^{-1}\right) 733$, 785, 801, 819, 915, 1013, 1082, 1153, 1181, 1217, 1235, 1310, 1462, 1479, 1507, 1596, 1726, 2838, 2903, 2949, 3002; MS (GCMS) $\mathrm{m} / z 232.0\left(\mathrm{M}^{+}\right)$; HRMS $\mathrm{C}_{13} \mathrm{H}_{13} \mathrm{O}_{4}{ }^{+}\left(\mathrm{M}+\mathrm{H}^{+}\right)$Expected 233.0808, Found 233.0804 .

Methyl 2-methoxy-5-(5-methylfuran-2-yl)benzoate, 37<smiles>COc1ccc(-c2ccc(C)o2)cc1C(=O)O[Na]</smiles>

Prepared as described in General Procedure C: methyl 2-methoxybenzoate ( $29 \mu \mathrm{L}, 0.20 \mathrm{mmol}$ ), dibenzothiophene S-oxide (44 mg, $0.22 \mathrm{mmol}$ ), $\mathrm{Tf}_{2} \mathrm{O}$ (41 $\left.\mu \mathrm{L}, 0.24 \mathrm{mmol}\right), \mathrm{PTH}(5.5 \mathrm{mg}, 0.02 \mathrm{mmol}$ ), 2methylfuran $(0.36 \mathrm{~mL}, 4.00 \mathrm{mmol}, 20$ equiv.), after purification by column chromatography on silica gel (10\% EtOAc in hexanes), gave 37 (25.9 mg, $0.11 \mathrm{mmol}, 53 \%)$ as a white solid; m.p. (recrystallized from $\left.\mathrm{CHCl}_{3}\right) 77-79{ }^{\circ} \mathrm{C} ; \delta_{\mathrm{H}}\left(500 \mathrm{MHz}, \mathrm{CDCl}_{3}\right) 2.36\left(\mathrm{~s}, 3 \mathrm{H}, \mathrm{CH}_{3}\right), 3.92\left(\mathrm{~s}, 3 \mathrm{H}, \mathrm{OCH}_{3}\right), 3.92\left(\mathrm{~s}, 3 \mathrm{H}, \mathrm{OCH}_{3}\right)$, $6.03(\mathrm{~d}, J=3.2 \mathrm{~Hz}, 1 \mathrm{H}, \operatorname{Ar} H), 6.45(\mathrm{~d}, J=3.2 \mathrm{~Hz}, 1 \mathrm{H}, \operatorname{Ar} H), 6.98(\mathrm{~d}, J=8.8 \mathrm{~Hz}, 1 \mathrm{H}, \operatorname{Ar} H), 7.71(\mathrm{dd}, J=$ 8.8, 2.4 Hz, 1H, $\operatorname{Ar~H}), 8.04(\mathrm{~d}, J=2.4 \mathrm{~Hz}, 1 \mathrm{H}, \mathrm{Ar} \mathrm{H}) ; \delta_{\mathrm{C}}\left(125 \mathrm{MHz}, \mathrm{CDCl}_{3}\right) 13.8\left(\mathrm{CH}_{3}\right), 52.3\left(\mathrm{OCH}_{3}\right), 56.3$ $\left(\mathrm{OCH}_{3}\right), 105.2(\mathrm{ArCH}), 107.8(\mathrm{ArCH}), 112.5(\mathrm{ArCH}), 120.3(\operatorname{ArC}), 124.1(\operatorname{ArC}), 126.9(\mathrm{ArCH}), 128.4(\mathrm{Ar}$ $C H), 151.3(\operatorname{Ar} C), 151.9(\operatorname{Ar} C), 158.0(\operatorname{Ar} C), 166.7(C=0) ; v_{\max }\left(\right.$ thin film/ $\left./ \mathrm{cm}^{-1}\right)$ 782, 819, 956, 1020, $1087,1153,1181,1233,1271,1312,1462,1489,1579,1728,2838,2918,2948,3000$; MS (GCMS) $\mathrm{m} / \mathrm{z} 246.1\left(\mathrm{M}^{+}\right)$; $\mathrm{HRMS} \mathrm{C}_{14} \mathrm{H}_{15} \mathrm{O}_{4}^{+}\left(\mathrm{M}+\mathrm{H}^{+}\right)$Expected 247.0965, Found 247.0962.

Methyl 5-(5-acetylfuran-2-yl)-2-methoxybenzoate, 38<smiles>COc1ccc(-c2ccc(C(C)=O)o2)cc1C(C)=O</smiles>

Prepared as described in General Procedure C: methyl 2-methoxybenzoate ( $29 \mu \mathrm{L}, 0.20 \mathrm{mmol}$ ), dibenzothiophene $S$-oxide (44 mg, $0.22 \mathrm{mmol}$ ), $\mathrm{Tf}_{2} \mathrm{O}$ (41 $\mu \mathrm{L}, 0.24 \mathrm{mmol}$ ), PTH (5.5 mg, $0.02 \mathrm{mmol}$ ), 2acetylfuran ( $0.40 \mathrm{~mL}, 4.00 \mathrm{mmol}, 20$ equiv.), after purification by column chromatography on silica 
gel (40\% EtOAc in hexanes), gave $38(38.4 \mathrm{mg}, 0.14 \mathrm{mmol}, 70 \%)$ as a white solid; m.p. (recrystallized from $\left.\mathrm{CHCl}_{3}\right) 94-96^{\circ} \mathrm{C} ; \delta_{\mathrm{H}}\left(400 \mathrm{MHz}, \mathrm{CDCl}_{3}\right) 2.51\left(\mathrm{~s}, 3 \mathrm{H}, \mathrm{CH}_{3}\right), 3.92\left(\mathrm{~s}, 3 \mathrm{H}, \mathrm{OCH}_{3}\right), 3.94\left(\mathrm{~s}, 3 \mathrm{H}, \mathrm{OCH}_{3}\right)$, $6.70(\mathrm{~d}, J=3.7 \mathrm{~Hz}, 1 \mathrm{H}, \operatorname{Ar} H), 7.03(\mathrm{dd}, J=8.8,2.2 \mathrm{~Hz}, 1 \mathrm{H}, \operatorname{Ar} H), 7.24(\mathrm{~d}, J=3.7 \mathrm{~Hz}, 1 \mathrm{H}, \operatorname{Ar} H), 7.89$ (dd, $J=8.8,2.2 \mathrm{~Hz}, 1 \mathrm{H}, \operatorname{Ar} H), 8.17(\mathrm{~d}, J=2.2 \mathrm{~Hz}, 1 \mathrm{H}, \operatorname{Ar} H) ; \delta_{\mathrm{C}}\left(100 \mathrm{MHz}, \mathrm{CDCl}_{3}\right) 26.1\left(\mathrm{CH}_{3}\right), 52.4\left(\mathrm{OCH}_{3}\right)$, $56.4\left(\mathrm{OCH}_{3}\right), 106.8(\mathrm{ArCH}), 112.6(\mathrm{ArCH}), 119.9(\mathrm{ArCH}), 120.8(\operatorname{ArC}), 122.0(\operatorname{ArC}), 128.6(\operatorname{ArCH})$, 130.1 ( $\operatorname{ArCH}), 151.8(\operatorname{ArC}), 156.9(\operatorname{Ar} C), 159.7(\operatorname{ArC}), 166.2(C=0), 186.2(C=0) ; v_{\max }\left(\operatorname{thin} \mathrm{film} / \mathrm{cm}^{-1}\right.$ ) 786, 817, 924, 971, 1018, 1074, 1088, 1156, 1182, 1233, 1273, 1311, 1409, 1434, 1475, 1581, 1613, 1727, 2839, 2949, 3002; MS (GCMS) $m / z 274.1\left(\mathrm{M}^{+}\right)$; HRMS $\mathrm{C}_{15} \mathrm{H}_{15} \mathrm{O}_{5}{ }^{+}\left(\mathrm{M}+\mathrm{H}^{+}\right)$Expected 275.0914, Found 275.0910 .

Methyl 5-(4-bromofuran-2-yl)-2-methoxybenzoate, 39<smiles>COc1ccc(-c2cc(Br)co2)cc1C(=O)O</smiles>

Prepared as described in General Procedure C: methyl 2-methoxybenzoate (29 $\mu \mathrm{L}, 0.20 \mathrm{mmol}$ ), dibenzothiophene $S$-oxide (44 mg, $0.22 \mathrm{mmol}$ ), Tf $\mathrm{T}_{2} \mathrm{O}$ (41 $\mu \mathrm{L}, 0.24 \mathrm{mmol}$ ), PTH (5.5 mg, $0.02 \mathrm{mmol}$ ), 3bromofuran ( $0.36 \mathrm{~mL}, 4.00 \mathrm{mmol}, 20$ equiv.), after purification by column chromatography on silica gel (10\% EtOAc in hexanes), gave $39(38.9 \mathrm{mg}, 0.13 \mathrm{mmol}, 63 \%)$ as a white solid; m.p. (recrystallized from $\left.\mathrm{CHCl}_{3}\right) 67-69^{\circ} \mathrm{C} ; \delta_{\mathrm{H}}\left(500 \mathrm{MHz}, \mathrm{CDCl}_{3}\right) 3.92\left(\mathrm{~s}, 3 \mathrm{H}, \mathrm{OCH}_{3}\right), 3.94\left(\mathrm{~s}, 3 \mathrm{H}, \mathrm{OCH}_{3}\right), 6.52(\mathrm{~d}, J=1.9 \mathrm{~Hz}$, $1 \mathrm{H}, \operatorname{Ar} H$ ), 7.04 (d, J=8.8 Hz, 1H, Ar H), 7.39 (d, J = 1.9 Hz, 1H, Ar H), 8.07 (dd, J = 8.8, 2.4 Hz, 1H, Ar $H), 8.38(\mathrm{~d}, J=2.4 \mathrm{~Hz}, 1 \mathrm{H}, \operatorname{Ar} H) ; \delta_{\mathrm{C}}\left(125 \mathrm{MHz}, \mathrm{CDCl}_{3}\right) 52.4\left(\mathrm{OCH}_{3}\right), 56.3\left(\mathrm{OCH}_{3}\right), 95.4(\operatorname{ArC}), 112.2(\operatorname{Ar}$ $\mathrm{CH}), 116.3(\mathrm{ArCH}), 120.2(\operatorname{ArC}), 122.4(\mathrm{ArC}), 129.2(\mathrm{ArCH}), 130.7(\mathrm{ArCH}), 141.7(\mathrm{ArCH}), 148.1(\mathrm{Ar}$ C), $158.8(\operatorname{ArC}), 166.4(C=0) ; v_{\max }\left(\right.$ thin film $\left./ \mathrm{cm}^{-1}\right)$ 738, 785, 818, 885, 950, 1022, 1084, 1183, 1226, $1265,1309,1462,1483,1511,1572,1615,1727,2838,2906,2948,3001$; MS (GCMS) m/z 310.0 $\left(\mathrm{M}^{+}\right) ; \mathrm{HRMS} \mathrm{C}_{13} \mathrm{H}_{12} \mathrm{O}_{4} \mathrm{Br}^{+}\left(\mathrm{M}+\mathrm{H}^{+}\right)$Expected 310.9913, Found 310.9911. 


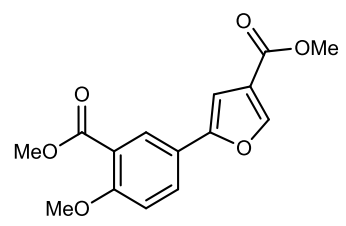

Prepared as described in General Procedure C: methyl 2-methoxybenzoate ( $29 \mu \mathrm{L}, 0.20 \mathrm{mmol}$ ), dibenzothiophene $S$-oxide ( $44 \mathrm{mg}, 0.22 \mathrm{mmol}$ ), $\mathrm{Tf}_{2} \mathrm{O}(41 \mu \mathrm{L}, 0.24 \mathrm{mmol}$ ), PTH (5.5 mg, $0.02 \mathrm{mmol}$ ), methyl furan-3-carboxylate $(0.43 \mathrm{~mL}, 4.00 \mathrm{mmol}, 20$ equiv.), after purification by column chromatography on silica gel (30\% EtOAc in hexanes), gave 40 (38.9 $\mathrm{mg}, 0.13 \mathrm{mmol}, 67 \%)$ as a white solid; m.p. (recrystallized from $\left.\mathrm{CHCl}_{3}\right) 89-91{ }^{\circ} \mathrm{C} ; \delta_{\mathrm{H}}\left(400 \mathrm{MHz}, \mathrm{CDCl}_{3}\right) 3.83\left(\mathrm{~s}, 3 \mathrm{H}, \mathrm{OCH}_{3}\right), 3.91(\mathrm{~s}, 3 \mathrm{H}$, $\left.\mathrm{OCH}_{3}\right), 3.95\left(\mathrm{~s}, 3 \mathrm{H}, \mathrm{OCH}_{3}\right), 6.81(\mathrm{~d}, J=2.0 \mathrm{~Hz}, 1 \mathrm{H}, \operatorname{Ar} H), 7.04(\mathrm{~d}, J=8.8 \mathrm{~Hz}, 1 \mathrm{H}, \operatorname{Ar} H), 7.39$ (d, J = 2.0 $\mathrm{Hz}, 1 \mathrm{H}, \operatorname{Ar} H), 8.21(\mathrm{dd}, J=8.8,2.4 \mathrm{~Hz}, 1 \mathrm{H}, \operatorname{Ar} H), 8.44(\mathrm{~d}, J=2.4 \mathrm{~Hz}, 1 \mathrm{H}, \operatorname{Ar} H) ; \delta_{\mathrm{c}}\left(100 \mathrm{MHz}, \mathrm{CDCl}_{3}\right)$ $51.8\left(\mathrm{OCH}_{3}\right), 52.3\left(\mathrm{OCH}_{3}\right), 56.3\left(\mathrm{OCH}_{3}\right), 111.7(\mathrm{ArCH}), 113.0(\operatorname{ArCH}), 113.1(\operatorname{ArC}), 119.8(\operatorname{ArC}), 122.1$ $(\operatorname{ArC}), 132.0(\operatorname{ArCH}), 133.7(\operatorname{ArCH}), 141.1(\operatorname{ArCH}), 156.7(\operatorname{Ar~C}), 159.9(\operatorname{ArC}), 164.1(C=0), 166.3$ $(C=0) ; v_{\max }\left(\right.$ thin film/ $\mathrm{cm}^{-1}$ ) 745, 785, 821, 935, 1077, 1149, 1227, 1263, 1302, 1461, 1489, 1581, $1612,1714,2843,2950,3002 ; \mathrm{MS}(\mathrm{GCMS}) \mathrm{m} / \mathrm{z} 290.1\left(\mathrm{M}^{+}\right)$; HRMS $\mathrm{C}_{15} \mathrm{H}_{14} \mathrm{O}_{6}{ }^{+}\left(\mathrm{M}^{+}\right)$Expected 290.0785, Found 290.0781 .

Methyl 5-(benzofuran-2-yl)-2-methoxybenzoate, 41<smiles>COc1ccc(-c2cc3ccccc3o2)cc1C(=O)O</smiles>

Prepared as described in General Procedure C: methyl 2-methoxybenzoate ( $29 \mu \mathrm{L}, 0.20 \mathrm{mmol}$ ), dibenzothiophene $S$-oxide (44 mg, $0.22 \mathrm{mmol}$ ), $\mathrm{Tf}_{2} \mathrm{O}$ ( $41 \mu \mathrm{L}, 0.24 \mathrm{mmol}$ ), PTH (5.5 mg, $0.02 \mathrm{mmol}$ ), benzofuran ( $0.44 \mathrm{~mL}, 4.00 \mathrm{mmol}, 20$ equiv.), after purification by column chromatography on silica gel (20\% EtOAc in hexanes), gave $41(29.9 \mathrm{mg}, 0.11 \mathrm{mmol}, 53 \%)$ as a white solid; m.p. (recrystallized from $\left.\mathrm{CHCl}_{3}\right) 110-112{ }^{\circ} \mathrm{C} ; \delta_{\mathrm{H}}\left(400 \mathrm{MHz}, \mathrm{CDCl}_{3}\right) 3.95\left(\mathrm{~s}, 3 \mathrm{H}, \mathrm{OCH}_{3}\right), 3.96\left(\mathrm{~s}, 3 \mathrm{H}, \mathrm{OCH}_{3}\right), 6.96(\mathrm{~d}, J=1.0$ $\mathrm{Hz}, 1 \mathrm{H}, \operatorname{Ar} H), 7.06(\mathrm{~d}, J=8.8 \mathrm{~Hz}, 1 \mathrm{H}, \operatorname{Ar} H), 7.23(\mathrm{td}, J=7.4,1.0 \mathrm{~Hz}, 1 \mathrm{H}, \operatorname{Ar} H), 7.25-7.30(\mathrm{~m}, 1 \mathrm{H}, \operatorname{Ar}$ H), $7.49-7.54(\mathrm{~m}, 1 \mathrm{H}, \operatorname{Ar} H), 7.54-7.60(\mathrm{~m}, 1 \mathrm{H}, \operatorname{Ar} H), 7.96(\mathrm{dd}, J=8.8,2.4 \mathrm{~Hz}, 1 \mathrm{H}, \operatorname{Ar} H), 8.30(\mathrm{~d}, J=$ $2.4 \mathrm{~Hz}, 1 \mathrm{H}, \mathrm{Ar} \mathrm{H}) ; \delta_{\mathrm{C}}\left(100 \mathrm{MHz}, \mathrm{CDCl}_{3}\right) 52.4\left(\mathrm{OCH}_{3}\right), 56.4\left(\mathrm{OCH}_{3}\right), 100.7(\operatorname{ArCH}), 111.2(\operatorname{ArCH}), 112.5$ ( $\operatorname{ArCH}), 120.6(\operatorname{ArC}), 120.9(\operatorname{ArCH}), 123.1(\operatorname{ArC}), 123.1(\operatorname{Ar} C H), 124.3(\operatorname{ArCH}), 128.6(\operatorname{ArCH}), 129.4$ $(\operatorname{ArC}), 130.0(\operatorname{ArCH}), 154.9(\operatorname{ArC}), 155.0(\operatorname{ArC}), 159.4(\operatorname{Ar} C), 166.4(C=0) ; v_{\max }\left(\right.$ thin film $\left./ \mathrm{cm}^{-1}\right)$ 749, 798, 929, 1021, 1082, 1182, 1204, 1237, 1273, 1350, 1434, 1452, 1496, 1595, 1615, 1729, 2837, 
2901, 2948, 3003; MS (GCMS) $\mathrm{m} / \mathrm{z} 282.1\left(\mathrm{M}^{+}\right)$; HRMS $\mathrm{C}_{17} \mathrm{H}_{14} \mathrm{O}_{4}^{+}\left(\mathrm{M}^{+}\right)$Expected 282.0887, Found 282.0895 .

Methyl 2-methoxy-5-(3-methyl-1H-indol-2-yl)benzoate, 42<smiles>COc1ccc(-c2[nH]c3ccccc3c2C)cc1C(=O)O[Na]</smiles>

Prepared as described in General Procedure C: methyl 2-methoxybenzoate ( $29 \mu \mathrm{L}, 0.20 \mathrm{mmol}$ ), dibenzothiophene $S$-oxide (44 mg, $0.22 \mathrm{mmol}$ ), $\mathrm{Tf}_{2} \mathrm{O}$ (41 $\left.\mu \mathrm{L}, 0.24 \mathrm{mmol}\right), \mathrm{PTH}(5.5 \mathrm{mg}, 0.02 \mathrm{mmol}), 3-$ methylindole ( $525 \mathrm{mg}, 4.00 \mathrm{mmol}, 20$ equiv.), after purification by column chromatography on silica gel (30\% EtOAc in hexanes), gave 42 (30.8 mg, $0.10 \mathrm{mmol}, 52 \%)$ as a white solid; m.p. (recrystallized from $\left.\mathrm{CHCl}_{3}\right) 131-133{ }^{\circ} \mathrm{C} ; \delta_{\mathrm{H}}\left(400 \mathrm{MHz}, \mathrm{CDCl}_{3}\right) 2.44\left(\mathrm{~s}, 3 \mathrm{H}, \mathrm{CH}_{3}\right), 3.93\left(\mathrm{~s}, 3 \mathrm{H}, \mathrm{OCH}_{3}\right), 3.97(\mathrm{~s}, 3 \mathrm{H}$, $\left.\mathrm{OCH}_{3}\right), 7.09(\mathrm{~d}, J=8.7 \mathrm{~Hz}, 1 \mathrm{H}, \operatorname{Ar} H), 7.15(\mathrm{t}, J=8.0 \mathrm{~Hz}, 1 \mathrm{H}, \operatorname{Ar} H), 7.21(\mathrm{t}, J=8.0 \mathrm{~Hz}, 1 \mathrm{H}, \operatorname{Ar} H), 7.36(\mathrm{~d}$, $J=8.0 \mathrm{~Hz}, 1 \mathrm{H}, \operatorname{Ar} H), 7.59(\mathrm{~d}, J=8.0 \mathrm{~Hz}, 1 \mathrm{H}, \operatorname{Ar} H), 7.69(\mathrm{dd}, J=8.7,2.4 \mathrm{~Hz}, 1 \mathrm{H}, \operatorname{Ar} H), 8.01(\mathrm{~d}, J=2.4$ $\mathrm{Hz}, 1 \mathrm{H}, \mathrm{Ar} H), 8.02(\mathrm{~s}, 1 \mathrm{H}, \mathrm{NH}) ; \delta_{\mathrm{C}}(100 \mathrm{MHz}, \mathrm{CDCl}) 9.7\left(\mathrm{CH}_{3}\right), 52.4\left(\mathrm{OCH}_{3}\right), 56.4\left(\mathrm{OCH}_{3}\right), 108.6(\operatorname{ArC})$, $110.8(\operatorname{ArCH}), 112.6(\operatorname{ArCH}), 119.0(\operatorname{ArCH}), 119.7(\operatorname{ArCH}), 120.6(\operatorname{ArC}), 122.4(\operatorname{ArCH}), 125.7(\operatorname{ArC})$, $130.1(\operatorname{ArC}), 130.9(\operatorname{ArCH}), 132.9(\operatorname{ArCH}), 133.0(\operatorname{ArC}), 135.9(\operatorname{ArC}), 158.5(\operatorname{ArC}), 166.6(C=0) ; v_{\max }$ (thin film/cm ${ }^{-1}$ ) 743, 821, 1019, 1087, 1182, 1242, 1261, 1307, 1460, 1489, 1501, 1574, 1613, 1714, 2840, 2861, 2948, 3003, 3360; MS (GCMS) $\mathrm{m} / \mathrm{z} 295.1\left(\mathrm{M}^{+}\right)$; HRMS $\mathrm{C}_{18} \mathrm{H}_{17} \mathrm{O}_{3} \mathrm{~N}^{+}\left(\mathrm{M}^{+}\right)$Expected 295.1203, Found 295.1206.

Methyl 2-methoxy-5-(pyrazin-2-yl)benzoate, 43<smiles>COc1ccc(-c2cnccn2)cc1C(=O)O</smiles>

Prepared as described in General Procedure C: methyl 2-methoxybenzoate ( $29 \mu \mathrm{L}, 0.20 \mathrm{mmol}$ ), dibenzothiophene $S$-oxide ( $44 \mathrm{mg}, 0.22 \mathrm{mmol}$ ), $\mathrm{Tf}_{2} \mathrm{O}(41 \mu \mathrm{L}, 0.24 \mathrm{mmol}$ ), PTH (5.5 mg, $0.02 \mathrm{mmol}$ ), pyrazine (320 mg, $4.00 \mathrm{mmol}, 20$ equiv.), after purification by column chromatography on silica gel (50\% EtOAc in hexanes), gave $43(20.5 \mathrm{mg}, 0.08 \mathrm{mmol}, 42 \%)$ as a white solid; m.p. (recrystallized from $\left.\mathrm{CHCl}_{3}\right) 86-88^{\circ} \mathrm{C} ; \delta_{\mathrm{H}}\left(400 \mathrm{MHz}, \mathrm{CDCl}_{3}\right) 3.93\left(\mathrm{~s}, 3 \mathrm{H}, \mathrm{OCH}_{3}\right), 3.97\left(\mathrm{~s}, 3 \mathrm{H}, \mathrm{OCH}_{3}\right), 7.11(\mathrm{~d}, \mathrm{~J}=8.8 \mathrm{~Hz}$, $1 \mathrm{H}, \operatorname{Ar} H$ ), 8.16 (dd, $J=8.8,2.5 \mathrm{~Hz}, 1 \mathrm{H}, \operatorname{Ar} H), 8.41-8.52(\mathrm{~m}, 2 \mathrm{H}, \operatorname{Ar} H), 8.59$ (dd, J = 2.5, $1.5 \mathrm{~Hz}, 1 \mathrm{H}, \operatorname{Ar}$ H), $9.01(\mathrm{~d}, J=1.5 \mathrm{~Hz}, 1 \mathrm{H}, \operatorname{Ar} H) ; \delta_{\mathrm{C}}\left(100 \mathrm{MHz}, \mathrm{CDCl}_{3}\right) 52.3\left(\mathrm{OCH}_{3}\right), 56.4\left(\mathrm{OCH}_{3}\right), 112.7(\operatorname{ArCH}), 120.7$ 
( $\operatorname{Ar} C), 128.5(\operatorname{ArC}), 130.4(\operatorname{ArCH}), 132.0(\operatorname{ArCH}), 141.7(\operatorname{ArCH}), 142.8(\operatorname{Ar~CH}), 144.2(\operatorname{ArCH}), 151.6$ $(\operatorname{ArC}), 160.6(\operatorname{Ar} C), 166.3(C=0) ; v_{\max }\left(\right.$ thin film $\left./ \mathrm{cm}^{-1}\right)$ 818, 969, 1013, 1041, 1076, 1093, 1142, 1184, 1229, 1274, 1410, 1435, 1504, 1609, 1725, 2840, 2949, 3003; MS (GCMS) m/z $244.1\left(\mathrm{M}^{+}\right)$; HRMS $\mathrm{C}_{13} \mathrm{H}_{13} \mathrm{O}_{3} \mathrm{~N}_{2}^{+}\left(\mathrm{M}+\mathrm{H}^{+}\right)$Expected 245.0921, Found 245.0917.

Methyl 5-(2,6-dimethoxypyridin-3-yl)-2-methoxybenzoate, 44<smiles>COc1ccc(-c2ccc(OC)c(C(=O)O[Na])c2)c(OC)n1</smiles>

Prepared as described in General Procedure C: methyl 2-methoxybenzoate ( $29 \mu \mathrm{L}, 0.20 \mathrm{mmol}$ ), dibenzothiophene $S$-oxide ( $44 \mathrm{mg}, 0.22 \mathrm{mmol}$ ), $\mathrm{Tf}_{2} \mathrm{O}$ ( $41 \mu \mathrm{L}, 0.24 \mathrm{mmol}$ ), PTH (5.5 mg, $0.02 \mathrm{mmol}$ ), 2,6-dimethoxypyridine $(0.53 \mathrm{~mL}, 4.00 \mathrm{mmol}, 20$ equiv.), after purification by column chromatography on silica gel (20\% EtOAc in hexanes), gave $44(25.6 \mathrm{mg}, 0.08 \mathrm{mmol}, 42 \%)$ as a colourless oil; $\delta_{\mathrm{H}}\left(400 \mathrm{MHz}, \mathrm{CDCl}_{3}\right) 3.90\left(\mathrm{~s}, 3 \mathrm{H}, \mathrm{OCH}_{3}\right), 3.93\left(\mathrm{~s}, 3 \mathrm{H}, \mathrm{OCH}_{3}\right), 3.96\left(\mathrm{~s}, 3 \mathrm{H}, \mathrm{OCH}_{3}\right), 3.96(\mathrm{~s}$, $\left.3 \mathrm{H}, \mathrm{OCH}_{3}\right), 6.38(\mathrm{~d}, J=8.0 \mathrm{~Hz}, 1 \mathrm{H}, \operatorname{Ar} H), 7.01(\mathrm{~d}, J=8.7 \mathrm{~Hz}, 1 \mathrm{H}, \operatorname{Ar} H), 7.55(\mathrm{~d}, J=8.0 \mathrm{~Hz}, 1 \mathrm{H}, \operatorname{Ar} H)$, $7.66(\mathrm{dd}, J=8.7,2.4 \mathrm{~Hz}, 1 \mathrm{H}, \operatorname{Ar} H), 7.94(\mathrm{~d}, J=2.4 \mathrm{~Hz}, 1 \mathrm{H}, \operatorname{Ar} H) ; \delta_{\mathrm{C}}\left(100 \mathrm{MHz}, \mathrm{CDCl}_{3}\right) 52.2\left(\mathrm{OCH}_{3}\right), 53.6$ $\left(\mathrm{OCH}_{3}\right), 53.8\left(\mathrm{OCH}_{3}\right), 56.3\left(\mathrm{OCH}_{3}\right), 101.2(\mathrm{ArCH}), 112.0(\mathrm{ArCH}), 114.4(\operatorname{ArC}), 119.9(\operatorname{ArC}), 129.1(\operatorname{ArC})$, 132.2 (ArCH), $134.1(\operatorname{ArCH}), 141.3(\operatorname{Ar} C H), 158.1(\operatorname{Ar} C), 159.3(\operatorname{Ar} C), 162.3(\operatorname{Ar} C), 166.8(C=0) ; v_{\max }$ (thin film/cm ${ }^{-1}$ ) 733, 808, 911, 953, 967, 1014, 1082, 1155, 1181, 1243, 1369, 1420, 1455, 1476, $1568,1597,1728,2836,2946,3005 ; \mathrm{MS}(\mathrm{GCMS}) \mathrm{m} / \mathrm{z} 303.1\left(\mathrm{M}^{+}\right)$; HRMS $\mathrm{C}_{16} \mathrm{H}_{18} \mathrm{O}_{5} \mathrm{~N}^{+}\left(\mathrm{M}+\mathrm{H}^{+}\right)$Expected 304.1179, Found 304.1170.

Methyl 4-methoxy-[1,1'-biphenyl]-3-carboxylate, 45<smiles>COc1ccc(-c2ccccc2)cc1C(C)=O</smiles>

Prepared as described in General Procedure C: methyl 2-methoxybenzoate ( $29 \mu \mathrm{L}, 0.20 \mathrm{mmol}$ ), dibenzothiophene $S$-oxide ( $44 \mathrm{mg}, 0.22 \mathrm{mmol}$ ), $\mathrm{Tf}_{2} \mathrm{O}$ (41 $\mu \mathrm{L}, 0.24 \mathrm{mmol}$ ), PTH (5.5 mg, $0.02 \mathrm{mmol}$ ), benzene (1.0 mL, $11.2 \mathrm{mmol}, 56$ equiv.), after purification by column chromatography on silica gel $\left(\mathrm{CHCl}_{3}\right)$, gave $45(26.6 \mathrm{mg}, 0.11 \mathrm{mmol}, 55 \%)$ as a colourless oil; $\delta_{\mathrm{H}}\left(400 \mathrm{MHz}, \mathrm{CDCl}_{3}\right) 3.92(\mathrm{~s}, 3 \mathrm{H}$, $\left.\mathrm{OCH}_{3}\right), 3.95\left(\mathrm{~s}, 3 \mathrm{H}, \mathrm{OCH}_{3}\right), 7.06(\mathrm{~d}, J=8.7 \mathrm{~Hz}, 1 \mathrm{H}, \operatorname{Ar} H), 7.30-7.36(\mathrm{~m}, 1 \mathrm{H}, \operatorname{Ar} H), 7.43(\mathrm{t}, J=7.6 \mathrm{~Hz}$, $2 \mathrm{H}, \operatorname{Ar} H), 7.54-7.59(\mathrm{~m}, 2 \mathrm{H}, \operatorname{Ar} H), 7.70(\mathrm{dd}, J=8.7,2.5 \mathrm{~Hz}, 1 \mathrm{H}, \operatorname{Ar} H), 8.05(\mathrm{~d}, J=2.5 \mathrm{~Hz}, 1 \mathrm{H}, \operatorname{Ar} H)$; 
$\delta_{\mathrm{C}}\left(100 \mathrm{MHz}, \mathrm{CDCl}_{3}\right) 52.3\left(\mathrm{OCH}_{3}\right), 56.3\left(\mathrm{OCH}_{3}\right), 112.6(\mathrm{ArCH}), 120.4(\mathrm{ArC}), 126.9(\mathrm{ArCH}), 127.3(\mathrm{Ar}$ $\mathrm{CH}), 129.0(\operatorname{ArCH}), 130.4(\operatorname{Ar} C H), 132.0(\operatorname{Ar} C H), 133.4(\operatorname{Ar} C), 139.9(\operatorname{Ar} C), 158.7(\operatorname{Ar} C), 166.8(C=0)$; $v_{\max }\left(\right.$ thin film/ $\left.\mathrm{cm}^{-1}\right) 761,820,968,1019,1082,1153,1181,1228,1272,1312,1433,1484,1583$, 1611, 1699, 1726, 2838, 2949, 3002; MS (GCMS) m/z $242.1\left(\mathrm{M}^{+}\right)$; $\mathrm{HRMS} \mathrm{C}_{15} \mathrm{H}_{15} \mathrm{O}_{3}{ }^{+}\left(\mathrm{M}+\mathrm{H}^{+}\right)$Expected 243.1016, Found 243.1015.

Methyl 2',5'-difluoro-4-methoxy-[1,1'-biphenyl]-3-carboxylate, 46<smiles>COC(=O)c1cc(-c2ccc(F)cc2F)ccc1OC</smiles>

Prepared as described in General Procedure C: methyl 2-methoxybenzoate ( $29 \mu \mathrm{L}, 0.20 \mathrm{mmol})$, dibenzothiophene $S$-oxide (44 mg, $0.22 \mathrm{mmol}$ ), $\mathrm{Tf}_{2} \mathrm{O}(41 \mu \mathrm{L}, 0.24 \mathrm{mmol}$ ), PTH (5.5 mg, $0.02 \mathrm{mmol}$ ), 1,4-difluorobenzene (1.0 mL, $9.7 \mathrm{mmol}, 49$ equiv.), after purification by column chromatography on silica gel $\left(\mathrm{CHCl}_{3}\right)$, gave $46(31.2 \mathrm{mg}, 0.11 \mathrm{mmol}, 56 \%)$ as a colourless oil; $\delta_{\mathrm{H}}\left(400 \mathrm{MHz}, \mathrm{CDCl}_{3}\right) 3.91(\mathrm{~s}$, $\left.3 \mathrm{H}, \mathrm{OCH})_{3}\right), 3.96\left(\mathrm{~s}, 3 \mathrm{H}, \mathrm{OCH}_{3}\right), 6.93-7.02(\mathrm{~m}, 1 \mathrm{H}, \operatorname{Ar} H), 7.06(\mathrm{~d}, J=8.7 \mathrm{~Hz}, 1 \mathrm{H}, \operatorname{Ar} H), 7.09-7.16(\mathrm{~m}$, $2 \mathrm{H}, \operatorname{Ar} H), 7.66(\mathrm{dt}, J=8.7,2.3 \mathrm{~Hz}, 1 \mathrm{H}, \operatorname{Ar} H), 7.98(\mathrm{dd}, J=2.3,1.2 \mathrm{~Hz}, 1 \mathrm{H}, \operatorname{Ar} H) ; \delta_{\mathrm{C}}\left(100 \mathrm{MHz}, \mathrm{CDCl}_{3}\right)$ $52.3\left(\mathrm{OCH}_{3}\right), 56.3\left(\mathrm{OCH}_{3}\right), 112.3(\mathrm{ArCH}), 115.2(\mathrm{dd}, J=23.9,8.6 \mathrm{~Hz}, \mathrm{ArCH}), 116.7$ (dd, $J=24.4,3.8 \mathrm{~Hz}$, $\operatorname{Ar} C H), 117.3(d d, J=25.9,8.8 \mathrm{~Hz}, \operatorname{Ar} C H), 120.3(\operatorname{ArC}), 126.9(\operatorname{ArC}), 129.1(\mathrm{dd}, J=15.8,8.1 \mathrm{~Hz}, \operatorname{Ar} C)$, 132.2 (d, J = 3.0 Hz, $\operatorname{ArCH}$ ), 134.0 (d, J = 3.9 Hz, $\operatorname{ArCH}$ ), 155.8 (dd, J = 243.4, 2.5 Hz, Ar C), 158.9 (dd, J = 242.6, $2.5 \mathrm{~Hz}, \operatorname{Ar} C), 159.2(\operatorname{Ar} C), 166.4(C=0) ; \delta_{\mathrm{F}}\left(376 \mathrm{MHz}, \mathrm{CDCl}_{3}\right)-124.3,-118.9 ; v_{\max }$ (thin film $/ \mathrm{cm}^{-1}$ ) 761, 820, 977, 1021, 1082, 1158, 1176, 1237, 1435, 1484, 1591, 1610, 1728, 2840, 2950, 3001; MS (GCMS) m/z $278.1\left(\mathrm{M}^{+}\right)$; HRMS $\mathrm{C}_{15} \mathrm{H}_{13} \mathrm{O}_{3} \mathrm{~F}_{2}^{+}\left(\mathrm{M}+\mathrm{H}^{+}\right)$Expected 279.0827, Found 279.0828.

Methyl 2',4,4',6'-tetramethoxy-[1,1'-biphenyl]-3-carboxylate, 47<smiles>COc1cc(OC)c(-c2ccc(OC)c(OC)c2)c(OC)c1</smiles>

Prepared as described in General Procedure C: methyl 2-methoxybenzoate ( $29 \mu \mathrm{L}, 0.20 \mathrm{mmol}$ ), dibenzothiophene $S$-oxide ( $44 \mathrm{mg}, 0.22 \mathrm{mmol}$ ), $\mathrm{Tf}_{2} \mathrm{O}$ (41 $\mu \mathrm{L}, 0.24 \mathrm{mmol}$ ), PTH (5.5 mg, $0.02 \mathrm{mmol}$ ), 1,3,5-trimethoxybenzene (673 mg, $4.00 \mathrm{mmol}, 20$ equiv.), after purification by column chromatography on silica gel (30\% EtOAc in hexanes), gave $47(34.7 \mathrm{mg}, 0.10 \mathrm{mmol}, 52 \%)$ as a white solid; m.p. (recrystallized from $\left.\mathrm{CHCl}_{3}\right) 46-48^{\circ} \mathrm{C} ; \delta_{\mathrm{H}}\left(400 \mathrm{MHz}, \mathrm{CDCl}_{3}\right) 3.72\left(\mathrm{~s}, 6 \mathrm{H}, \mathrm{OCH}_{3}\right), 3.86(\mathrm{~s}, 3 \mathrm{H}$, 
$\left.\mathrm{OCH}_{3}\right), 3.87\left(\mathrm{~s}, 3 \mathrm{H}, \mathrm{OCH}_{3}\right), 3.93\left(\mathrm{~s}, 3 \mathrm{H}, \mathrm{OCH}_{3}\right), 6.22(\mathrm{~s}, 2 \mathrm{H}, \operatorname{Ar} H), 7.00(\mathrm{~d}, J=8.6 \mathrm{~Hz}, 1 \mathrm{H}, \operatorname{Ar~H}), 7.44$ (dd, $J=8.6,2.3 \mathrm{~Hz}, 1 \mathrm{H}, \operatorname{Ar} H), 7.79(\mathrm{~d}, J=2.3 \mathrm{~Hz}, 1 \mathrm{H}, \operatorname{Ar} H) ; \delta_{\mathrm{C}}\left(100 \mathrm{MHz}, \mathrm{CDCl}_{3}\right) 52.0\left(\mathrm{OCH}_{3}\right), 55.5\left(\mathrm{OCH}_{3}\right)$, $56.0\left(\mathrm{OCH}_{3}\right), 56.0\left(\mathrm{OCH}_{3}\right), 56.1\left(\mathrm{OCH}_{3}\right), 91.0(\operatorname{ArCH}), 111.1(\operatorname{ArC}), 111.5(\operatorname{ArCH}), 119.3(\operatorname{ArC}), 126.1$ $(\operatorname{ArC}), 134.8(\operatorname{ArCH}), 136.5(\operatorname{ArCH}), 157.9(\operatorname{ArC}), 158.5(\operatorname{Ar} C), 160.7(\operatorname{ArC}), 166.9(C=0) ; v_{\max }(\operatorname{thin}$ film/cm ${ }^{-1}$ ) 733, 813, 947, 1021, 1068, 1082, 1121, 1152, 1220, 1267, 1307, 1434, 1583, 1602, 1725, 2837, 2944, 3000; MS (GCMS) $\mathrm{m} / z 332.2\left(\mathrm{M}^{+}\right)$; HRMS $\mathrm{C}_{18} \mathrm{H}_{21} \mathrm{O}_{6}{ }^{+}\left(\mathrm{M}+\mathrm{H}^{+}\right)$Expected 333.1333, Found 333.1327.

Methyl 2-methoxy-5-(2-methoxynaphthalen-1-yl)benzoate, 48<smiles>COC(=O)c1cc(-c2cccc3ccccc23)ccc1OC</smiles>

Prepared as described in General Procedure C: methyl 2-methoxybenzoate ( $29 \mu \mathrm{L}, 0.20 \mathrm{mmol}$ ), dibenzothiophene S-oxide ( $44 \mathrm{mg}, 0.22 \mathrm{mmol}$ ), $\mathrm{Tf}_{2} \mathrm{O}$ ( $41 \mu \mathrm{L}, 0.24 \mathrm{mmol}$ ), PTH (5.5 mg, $0.02 \mathrm{mmol}$ ), 2methoxynapthalene (633 $\mathrm{mg}, 4.00 \mathrm{mmol}, 20$ equiv.), after purification by column chromatography on silica gel $\left(\mathrm{CH}_{2} \mathrm{Cl}_{2}\right)$, gave $48(25.8 \mathrm{mg}, 0.08 \mathrm{mmol}, 40 \%)$ as a white solid; m.p. (recrystallized from $\left.\mathrm{CHCl}_{3}\right) 144-146^{\circ} \mathrm{C} ; \delta_{\mathrm{H}}\left(400 \mathrm{MHz}, \mathrm{CDCl}_{3}\right) 3.84\left(\mathrm{~s}, 3 \mathrm{H}, \mathrm{OCH}_{3}\right), 3.87\left(\mathrm{~s}, 3 \mathrm{H}, \mathrm{OCH}_{3}\right), 4.00\left(\mathrm{~s}, 3 \mathrm{H}, \mathrm{OCH}_{3}\right)$, $7.12(\mathrm{~d}, J=8.5 \mathrm{~Hz}, 1 \mathrm{H}, \operatorname{Ar} H), 7.33-7.38(\mathrm{~m}, 3 \mathrm{H}, \operatorname{Ar} H), 7.45-7.52(\mathrm{~m}, 2 \mathrm{H}, \operatorname{Ar} H), 7.80-7.85(\mathrm{~m}, 2 \mathrm{H}$, $\operatorname{Ar} H), 7.88(\mathrm{~d}, J=9.1 \mathrm{~Hz}, 1 \mathrm{H}, \mathrm{Ar} H) ; \delta_{\mathrm{C}}\left(100 \mathrm{MHz}, \mathrm{CDCl}_{3}\right) 52.1\left(\mathrm{OCH}_{3}\right), 56.3\left(\mathrm{OCH}_{3}\right), 56.9\left(\mathrm{OCH}_{3}\right), 112.1$ ( $\operatorname{ArCH}), 113.8(\operatorname{ArCH}), 119.9(\operatorname{ArC}), 123.7(\operatorname{ArCH}), 123.9(\operatorname{ArC}), 125.1(\operatorname{ArCH}), 126.6(\operatorname{ArCH}), 128.1$ ( $\operatorname{ArCH}), 128.3(\operatorname{ArC}), 129.2(\operatorname{ArC}), 129.4(\operatorname{ArCH}), 133.8(\operatorname{ArC}), 134.4(\operatorname{ArCH}), 136.3(\operatorname{ArCH}), 154.1(\operatorname{Ar}$ C), $158.5(\operatorname{Ar} C), 166.8(C=0) ; v_{\max }\left(\right.$ thin film $\left./ \mathrm{cm}^{-1}\right)$ 750, 809, 961, 1004, 1022, 1082, 1245, 1260, 1301, 1435, 1499, 1592, 1726, 2837, 2902, 2946, 3001; MS (GCMS) m/z $322.1\left(\mathrm{M}^{+}\right)$; HRMS $\mathrm{C}_{20} \mathrm{H}_{19} \mathrm{O}_{4}^{+}$ $\left(\mathrm{M}+\mathrm{H}^{+}\right)$Expected 323.1278, Found 323.1274. 


\section{Concise Synthesis of Pseudilins}

tert-Butyl 2-(3,5-dichloro-2-methoxyphenyl)-1H-pyrrole-1-carboxylate, S11<smiles>COc1c(Cl)cc(Cl)cc1-c1cccn1C(=O)OCc1ccccc1</smiles>

$\mathrm{Tf}_{2} \mathrm{O}$ (170 $\mu \mathrm{L}, 1.00 \mathrm{mmol}, 2.0$ equiv.) was slowly added to a stirred solution of 2,4-dichloroanisole (69 $\mu \mathrm{L}, 0.50 \mathrm{mmol}, 1.0$ equiv.) and dibenzothiophene $S$-oxide (200 mg, $1.00 \mathrm{mmol}, 2.0$ equiv.) in $\mathrm{CH}_{2} \mathrm{Cl}_{2}$ $(0.1 \mathrm{M})$ at $-40{ }^{\circ} \mathrm{C}$ under a nitrogen atmosphere. The resulting solution was stirred at this temperature for 2 hours before warming to room temperature. The solvent was removed in vacuo and PTH (13.7 mg, $0.05 \mathrm{mmol}, 10 \mathrm{~mol} \%$ ) was then added to the reaction vial, followed by evacuation and refilling with nitrogen ( 3 cycles). DMSO $(0.2 \mathrm{M})$ was then added followed by $\mathrm{N}$-Boc-pyrrole (835 $\mu \mathrm{L}, 5.00 \mathrm{mmol}, 10$ equiv.). The reaction mixture was then irradiated with a Kessil $34 \mathrm{~W}$ blue LED lamp for $18 \mathrm{~h}$ before quenching with aqueous saturated $\mathrm{NaHCO}_{3}$ and dilution with EtOAc. The organic layer was washed with brine, dried using $\mathrm{MgSO}_{4}$, filtered, and concentrated in vacuo, to give the crude product, which was purified by column chromatography $\left(50 \% \mathrm{CHCl}_{3} /\right.$ hexanes to $\left.\mathrm{CHCl}_{3}\right)$ to give S11 (135 mg, $0.39 \mathrm{mmol}, 79 \%)$ as a brown oil; $\delta_{\mathrm{H}}\left(400 \mathrm{MHz}, \mathrm{CDCl}_{3}\right) 1.32\left(\mathrm{~d}, J=1.2,9 \mathrm{H}, \mathrm{CH}_{3}\right), 3.44$ (d, $\left.J=1.2 \mathrm{~Hz}, 3 \mathrm{H}, \mathrm{OCH}_{3}\right), 6.19-6.29(\mathrm{~m}, 2 \mathrm{H}, \operatorname{Ar} H), 7.20(\mathrm{dd}, J=2.6,1.1 \mathrm{~Hz}, 1 \mathrm{H}, \operatorname{Ar} H), 7.35(\mathrm{dd}, J=2.6$, $1.1 \mathrm{~Hz}, 1 \mathrm{H}, \operatorname{Ar} H), 7.42(\mathrm{dt}, J=3.0,1.4 \mathrm{~Hz}, 1 \mathrm{H}, \operatorname{Ar} H) . \delta_{\mathrm{C}}\left(100 \mathrm{MHz}, \mathrm{CDCl}_{3}\right) 27.6\left(\mathrm{CH}_{3}\right), 60.3\left(\mathrm{OCH}_{3}\right)$, $84.1\left(\mathrm{CH}_{3} \mathrm{C}\right), 110.6(\operatorname{ArCH}), 115.2(\mathrm{Ar} \mathrm{CH}), 123.2(\operatorname{ArCH}), 128.1(\operatorname{ArC}), 128.4(\operatorname{Ar~C}), 128.8(\operatorname{Ar~CH})$, $128.9(\operatorname{ArC}), 129.1(\operatorname{ArCH}), 131.7(\operatorname{ArC}), 152.8(\operatorname{ArC}), C=0$ not observed; $v_{\max }\left(\right.$ thin film $/ \mathrm{cm}^{-1}$ ) 732, 842, 1002, 1147, 1312, 1337, 1370, 1742, 2935, 2978; MS (APCI) $m / z 342.1\left(\mathrm{M}+\mathrm{H}^{+}\right)$; HRMS $\mathrm{C}_{16} \mathrm{H}_{18} \mathrm{Cl}_{2} \mathrm{NO}_{3}{ }^{+}\left(\mathrm{M}+\mathrm{H}^{+}\right)$Expected 342.0658, Found 342.0657.

\section{2-(3,5-Dichloro-2-methoxyphenyl)-1H-pyrrole, 49}<smiles>COc1c(Cl)cc(Cl)cc1-c1ccc[nH]1</smiles>

A solution of $\mathrm{NaOMe}(1.0 \mathrm{~mL}, 30 \% \mathrm{w} / \mathrm{w}$ in $\mathrm{MeOH})$ was added to a solution of tert-butyl 2-(3,5dichloro-2-methoxyphenyl)-1H-pyrrole-1-carboxylate, $\mathbf{S 1 1}(171 \mathrm{mg}, 0.50 \mathrm{mmol})$ in anhydrous THF $\left(3.5 \mathrm{~mL}\right.$ ) and the resulting mixture was heated at $45^{\circ} \mathrm{C}$ for 2 hours. The reaction was allowed to cool to room temperature and was quenched by the addition of $\mathrm{H}_{2} \mathrm{O}(5 \mathrm{~mL})$. The mixture was extracted 
with $\mathrm{CH}_{2} \mathrm{Cl}_{2}(2 \times 5 \mathrm{~mL})$, the combined organic extracts were washed with $\mathrm{H}_{2} \mathrm{O}(3 \times 10 \mathrm{~mL})$, dried using $\mathrm{MgSO}_{4}$, filtered, and concentrated in vacuo. The resulting crude pyrrole 49 (117 mg, $0.48 \mathrm{mmol}$, 97\%) was used without purification; $\delta_{\mathrm{H}}\left(500 \mathrm{MHz}, \mathrm{CDCl}_{3}\right) 3.78\left(\mathrm{~s}, 3 \mathrm{H}, \mathrm{OCH}_{3}\right), 6.30(\mathrm{dt}, J=3.8,2.6 \mathrm{~Hz}$, $1 \mathrm{H}, \operatorname{Ar} H$ ), 6.61 (ddd, $J=3.8,2.6,1.5 \mathrm{~Hz}, 1 \mathrm{H}, \operatorname{Ar} H), 6.92(\mathrm{td}, J=2.8,1.5 \mathrm{~Hz}, 1 \mathrm{H}, \operatorname{Ar} H), 7.19$ (d, J = 2.5 $\mathrm{Hz}, 1 \mathrm{H}, \operatorname{Ar} H), 7.47(\mathrm{~d}, J=2.5 \mathrm{~Hz}, 1 \mathrm{H}, \mathrm{Ar} H), 9.67(\mathrm{~s}, 1 \mathrm{H}, \mathrm{NH}) ; \delta_{\mathrm{C}}\left(125 \mathrm{MHz}, \mathrm{CDCl}_{3}\right) 60.9\left(\mathrm{OCH}_{3}\right), 108.6$ $(\operatorname{ArCH}), 109.7(\operatorname{ArCH}), 119.9(\operatorname{ArCH}), 125.4(\operatorname{ArCH}), 127.1(\operatorname{ArCH}), 127.2(\operatorname{ArC}), 128.3(\operatorname{ArC}), 129.7$ $(\operatorname{ArC}), 130.3(\operatorname{Ar} C), 149.9(\operatorname{Ar} C)$.

The data is in accordance with the literature. ${ }^{15}$

2,4-dichloro-6-(3,4,5-trichloro-1H-pyrrol-2-yl)phenol, 50<smiles>Oc1c(Cl)cc(Cl)cc1-c1[nH]c(Cl)c(Cl)c1Cl</smiles>

A solution of $N$-chlorosuccinimide ( $46.7 \mathrm{mg}, 0.35 \mathrm{mmol}, 3.0$ equiv.) in $\mathrm{MeCN}(0.5 \mathrm{~mL})$ was added to a solution of 2-(3,5-dichloro-2-methoxyphenyl)-1H-pyrrole, 49 (30.3 mg, $0.12 \mathrm{mmol}, 1.0$ equiv.) in $\operatorname{MeCN}(2 \mathrm{~mL})$ at $-40{ }^{\circ} \mathrm{C}$. The reaction mixture was stirred at $-40^{\circ} \mathrm{C}$ for $15 \mathrm{~min}$ and then allowed to warm up to room temperature and was stirred for 15 hours. The solvent was removed in vacuo and the residue was filtered through a plug of silica, eluting with $50 \% \mathrm{CHCl}_{3} /$ hexanes. The resulting crude product $\left(0.07 \mathrm{mmol}, 55 \%\right.$, yield determined by ${ }^{1} \mathrm{H}$ NMR analysis) was dissolved in $\mathrm{CH}_{2} \mathrm{Cl}_{2}(2 \mathrm{~mL})$ and cooled to $-78^{\circ} \mathrm{C}$. Boron tribromide ( $1 \mathrm{M}$ in $\mathrm{CH}_{2} \mathrm{Cl}_{2}, 70 \mu \mathrm{L}, 0.07 \mathrm{mmol}, 1.1$ equiv.) was then added and the reaction mixture was stirred at $0{ }^{\circ} \mathrm{C}$ for 2 hours. The reaction was quenched by the addition of $\mathrm{MeOH}(5 \mathrm{~mL})$ and the solvent was removed in vacuo. To the residue was added $\mathrm{H}_{2} \mathrm{O}(5 \mathrm{~mL})$ and the product was extracted into $\mathrm{Et}_{2} \mathrm{O}(2 \times 5 \mathrm{~mL})$. The combined organic extracts were dried using $\mathrm{MgSO}_{4}$, filtered and concentrated in vacuo, to give the crude product which was purified by column chromatography on silica gel (20\% to $40 \% \mathrm{Et}_{2} \mathrm{O}$ in hexanes), to give $\mathbf{5 0}(13.7 \mathrm{mg}, 0.04 \mathrm{mmol}, 64 \%$ ) as a purple solid; $\delta_{\mathrm{H}}\left(400 \mathrm{MHz}, \mathrm{CDCl}_{3}\right) 6.12-6.24(1 \mathrm{H}, \mathrm{m}, \mathrm{OH}), 7.29(\mathrm{dd}, J=2.5,1.3 \mathrm{~Hz}, 1 \mathrm{H}, \mathrm{Ar} \mathrm{H}), 8.00$ (dd, J = 2.5, $1.3 \mathrm{~Hz}, 1 \mathrm{H}, \operatorname{Ar} H), 9.57(\mathrm{~s}, 1 \mathrm{H}, \mathrm{NH}) ; \delta_{\mathrm{C}}\left(100 \mathrm{MHz}, \mathrm{CDCl}_{3}\right) 110.6(\operatorname{ArC}), 110.6(\operatorname{ArC}), 112.5$ $(\operatorname{ArC}), 118.8(\operatorname{Ar} C), 120.7(\operatorname{Ar} C), 121.5(\operatorname{Ar} C), 126.6(\operatorname{ArCH}$ and $\operatorname{Ar} C), 127.3(\operatorname{ArCH}), 145.7(\operatorname{Ar} C)$.

The data is in accordance with the literature. ${ }^{15}$ 
<smiles>Oc1c(Cl)cc(Cl)cc1-c1[nH]c(Br)c(Br)c1Br</smiles>

Pyridinium tribromide (113 mg, $0.35 \mathrm{mmol}, 3.0$ equiv.) was added to a solution of 2-(3,5-dichloro-2methoxyphenyl)-1H-pyrrole, 49 (30.3 mg, $0.12 \mathrm{mmol}, 1.0$ equiv.) in $\mathrm{EtOH}(1 \mathrm{~mL})$ at room temperature and the mixture was stirred for $30 \mathrm{~min}$. The solvent was removed in vacuo and the residue was filtered through a plug of silica, eluting with $25 \%$ to $50 \% \mathrm{CHCl}_{3} /$ hexanes, to give $\mathbf{S 1 2}$ (39.7 mg, $0.08 \mathrm{mmol}, 70 \%$ ) as a brown oil; $\delta_{\mathrm{H}}\left(400 \mathrm{MHz}, \mathrm{CDCl}_{3}\right) 3.68\left(3 \mathrm{H}, \mathrm{s}, \mathrm{OCH}_{3}\right), 7.35(\mathrm{~d}, J=2.5 \mathrm{~Hz}$, $1 \mathrm{H}, \operatorname{Ar} H), 7.82(\mathrm{~d}, J=2.5 \mathrm{~Hz}, 1 \mathrm{H}, \mathrm{Ar} H), 9.41-9.64(\mathrm{~m}, 1 \mathrm{H}, \mathrm{NH}) ; \delta_{\mathrm{C}}\left(100 \mathrm{MHz}, \mathrm{CDCl}_{3}\right) 61.3\left(\mathrm{OCH}_{3}\right)$, 99.7 (ArC), $101.5(\operatorname{ArC}), 103.9(\operatorname{ArC}), 125.2(\operatorname{ArC}), 125.9(\operatorname{ArC}), 127.8(\operatorname{ArCH}), 129.7(\operatorname{ArCH}), 129.7$ $(\operatorname{ArC}), 130.3(\operatorname{Ar} C), 151.3(\operatorname{Ar} C)$.

The data is in accordance with the literature. ${ }^{15}$

\section{2,4-dichloro-6-(3,4,5-tribromo-1H-pyrrol-2-yl)phenol, 51}<smiles>Oc1c(Cl)cc(Cl)cc1-c1[nH]c(Br)c(Br)c1Br</smiles>

Boron tribromide ( $1 \mathrm{M}$ in $\mathrm{CH}_{2} \mathrm{Cl}_{2}, 60 \mu \mathrm{L}, 60.0 \mu \mathrm{mol}, 1.1$ equiv.) was added to a solution of 2,3,4tribromo-5-(3,5-dichloro-2-methoxyphenyl)-1H-pyrrole, $\mathbf{S 1 2}(27.0 \mathrm{mg}, 56.3 \mu \mathrm{mol})$ in $\mathrm{CH}_{2} \mathrm{Cl}_{2}(2 \mathrm{~mL})$ at $-78{ }^{\circ} \mathrm{C}$ and the reaction mixture was stirred at $0{ }^{\circ} \mathrm{C}$ for $2 \mathrm{~h}$. The reaction was quenched by the addition of $\mathrm{MeOH}(5 \mathrm{~mL})$ and the solvent was removed in vacuo. To the residue was added $\mathrm{H}_{2} \mathrm{O}(5$ $\mathrm{mL}$ ) and the product was extracted into $\mathrm{Et}_{2} \mathrm{O}(2 \times 5 \mathrm{~mL})$. The combined organic extracts were dried using $\mathrm{MgSO}_{4}$, filtered and concentrated in vacuo, to give $51(25.0 \mathrm{mg}, 53.8 \mu \mathrm{mol}, 96 \%)$ as an offwhite solid; $\delta_{\mathrm{H}}\left(400 \mathrm{MHz}, \mathrm{CDCl}_{3}\right) 6.10(1 \mathrm{H}, \mathrm{s}, \mathrm{OH}), 7.31(\mathrm{~d}, J=2.4 \mathrm{~Hz}, 1 \mathrm{H}, \mathrm{Ar} H), 7.96(\mathrm{~d}, J=2.4 \mathrm{~Hz}, 1$ H, $\operatorname{Ar} H), 9.55(\mathrm{~s}, 1 \mathrm{H}, \mathrm{NH}) ; \delta_{\mathrm{C}}\left(100 \mathrm{MHz}, \mathrm{CDCl}_{3}\right) 99.5(\operatorname{Ar} C), 101.2(\operatorname{ArC}), 104.1(\operatorname{ArC}), 119.2(\operatorname{Ar} C)$, 121.5 ( $\operatorname{ArC}), 125.3(\operatorname{ArC}), 126.3(\operatorname{ArC}), 127.4(\operatorname{ArCH}), 127.8(\operatorname{ArCH}), 146.1(\operatorname{ArC})$.

The data is in accordance with the literature. ${ }^{15}$ 


\section{Late-Stage Functionalization}

(2R,3R,4S,5R,6S)-2-(Acetoxymethyl)-6-(2-(acetoxymethyl)-4-(1-methyl-1H-pyrrol-2yl)phenoxy)tetrahydro-2H-pyran-3,4,5-triyl triacetate, 52

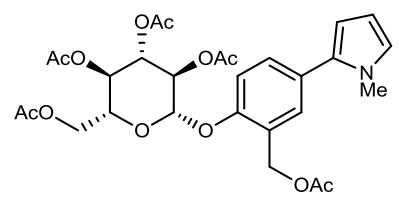

Prepared as described in General Procedure C: $\mathbf{S 9}$ (99 mg, $0.20 \mathrm{mmol}$ ), dibenzothiophene S-oxide (44 $\mathrm{mg}, 0.22 \mathrm{mmol}$ ), $\mathrm{Tf}_{2} \mathrm{O}(41 \mu \mathrm{L}, 0.24 \mathrm{mmol}), \mathrm{PTH}(5.5 \mathrm{mg}, 0.02 \mathrm{mmol}), N$-methylpyrrole $(0.36 \mathrm{~mL}, 4.00$ mmol, 20 equiv.), after purification by column chromatography on silica gel (40\% EtOAc in hexanes), gave $52(81.7 \mathrm{mg}, 0.14 \mathrm{mmol}, 71 \%)$ as a white gum; $\delta_{\mathrm{H}}\left(400 \mathrm{MHz}, \mathrm{CDCl}_{3}\right) 2.05\left(\mathrm{~s}, 3 \mathrm{H}, \mathrm{CH}_{3}\right), 2.05(\mathrm{~s}, 3 \mathrm{H}$, $\left.\mathrm{CH}_{3}\right), 2.08\left(\mathrm{~s}, 3 \mathrm{H}, \mathrm{CH}_{3}\right), 2.10\left(\mathrm{~s}, 3 \mathrm{H}, \mathrm{CH}_{3}\right), 2.11\left(\mathrm{~s}, 3 \mathrm{H}, \mathrm{CH}_{3}\right), 3.62$ (s, 3H, $\left.\mathrm{NCH}_{3}\right), 3.88$ (ddd, $J=10.0,5.3$, $2.5 \mathrm{~Hz}, 1 \mathrm{H}$, Alk $\mathrm{CH}$ ), 4.20 (dd, $J=12.3,2.5 \mathrm{~Hz}, 1 \mathrm{H}$, Alk $\mathrm{CH}_{2}$ ), 4.29 (dd, $J=12.3,5.3 \mathrm{~Hz}, 1 \mathrm{H}$, Alk $\mathrm{CH}_{2}$ ), $5.03-5.23\left(\mathrm{~m}, 4 \mathrm{H}, \mathrm{OCHO}\right.$, Alk CH \& Bn $\left.\mathrm{CH}_{2}\right), 5.27-5.37(\mathrm{~m}, 2 \mathrm{H}$, Alk $\mathrm{CH}), 6.14-6.22(\mathrm{~m}, 2 \mathrm{H}, \mathrm{Ar} H)$, $6.68-6.72(\mathrm{~m}, 1 \mathrm{H}, \operatorname{Ar} H), 7.10(\mathrm{~d}, J=8.5 \mathrm{~Hz}, 1 \mathrm{H}, \operatorname{Ar} H), 7.30(\mathrm{dd}, J=8.5,2.3 \mathrm{~Hz}, 1 \mathrm{H}, \operatorname{Ar} H), 7.37(\mathrm{~d}, J=$ $2.3 \mathrm{~Hz}, 1 \mathrm{H}, \mathrm{Ar} \mathrm{H}) ; \delta_{\mathrm{C}}(100 \mathrm{MHz}, \mathrm{CDCl})_{3} 20.7\left(\mathrm{CH}_{3}\right), 20.7\left(\mathrm{CH}_{3}\right), 20.7\left(\mathrm{CH}_{3}\right), 20.8\left(\mathrm{CH}_{3}\right), 21.1\left(\mathrm{CH}_{3}\right), 35.1$ $\left(\mathrm{NCH}_{3}\right.$ ), 61.1 (Alk $\mathrm{CH}_{2}$ ), 62.0 (Bn $\mathrm{CH}_{2}$ ), 68.4 (Alk CH), 71.1 (Alk $\mathrm{CH}$ ), 72.2 (Alk $\mathrm{CH}$ ), 72.7 (Alk CH), 99.4 (OCHO), 107.9 ( $\mathrm{ArCH}), 108.7$ ( $\mathrm{ArCH}), 115.8(\mathrm{ArCH}), 123.7$ (Ar CH), $126.3(\operatorname{ArC}), 128.9(\operatorname{ArC}), 129.7$ ( $\operatorname{ArCH}), 129.9(\operatorname{ArCH}), 133.7(\operatorname{Ar} C), 153.5(\operatorname{Ar} C), 169.4(C=0), 169.5(C=0), 170.3(C=0), 170.6(C=0)$, $170.8(C=0) ; v_{\max }\left(\right.$ thin film $\left./ \mathrm{cm}^{-1}\right) 733,833,907,983,1033,1121,1210,1365,1375,1498,1545$, 1741, 2958, 3056; MS (GCMS) $\mathrm{m} / z 575.3\left(\mathrm{M}^{+}\right)$; HRMS $\mathrm{C}_{28} \mathrm{H}_{33} \mathrm{O}_{12} \mathrm{NNa}^{+}\left(\mathrm{M}+\mathrm{Na}^{+}\right)$Expected 598.1895, Found 598.1866.

2-Chloro-N-(4'-chloro-5-(1-methyl-1H-pyrrol-2-yl)-[1,1'-biphenyl]-2-yl)nicotinamide, 53
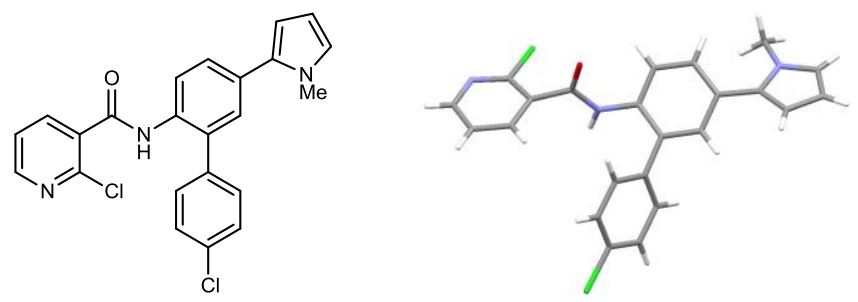

Prepared as described in General Procedure D: boscalid $(69 \mathrm{mg}, 0.20 \mathrm{mmol})$, dibenzothiophene $S$ oxide (44 mg, $0.22 \mathrm{mmol}), \mathrm{Tf}_{2} \mathrm{O}$ (41 $\mu \mathrm{L}, 0.24 \mathrm{mmol}$ ), PTH (5.5 mg, $\left.0.02 \mathrm{mmol}\right), N$-methylpyrrole (0.36 $\mathrm{mL}, 4.00 \mathrm{mmol}, 20$ equiv.), after purification by column chromatography on silica gel ( $40 \%$ EtOAc, $1 \%$ 
$\mathrm{Et}_{3} \mathrm{~N}$ in hexanes), gave 53 (55.7 mg, $\left.0.13 \mathrm{mmol}, 66 \%\right)$ as an off-white solid; m.p. (recrystallized from $\left.\mathrm{CHCl}_{3}\right) 145-147^{\circ} \mathrm{C} ; \delta_{\mathrm{H}}\left(400 \mathrm{MHz}, \mathrm{CDCl}_{3}\right) 3.71\left(\mathrm{~s}, 3 \mathrm{H}, \mathrm{NCH}_{3}\right), 6.21(\mathrm{dd}, J=3.6,2.7 \mathrm{~Hz}, 1 \mathrm{H}, \operatorname{Ar~H}), 6.26$ (dd, $J=3.6,1.8 \mathrm{~Hz}, 1 \mathrm{H}, \operatorname{Ar} H), 6.73(\mathrm{dd}, J=2.7,1.8 \mathrm{~Hz}, 1 \mathrm{H}, \operatorname{Ar} H), 7.32(\mathrm{~d}, J=2.1 \mathrm{~Hz}, 1 \mathrm{H}, \operatorname{Ar} H), 7.33-$ $7.41(\mathrm{~m}, 3 \mathrm{H}, \operatorname{Ar} H), 7.41-7.53(\mathrm{~m}, 3 \mathrm{H}, \operatorname{Ar} H), 8.17(\mathrm{dd}, J=7.7,2.1 \mathrm{~Hz}, 1 \mathrm{H}, \operatorname{Ar} H), 8.21(\mathrm{~s}, 1 \mathrm{H}, \mathrm{NH}), 8.43$ - $8.50(\mathrm{~m}, 2 \mathrm{H}, \mathrm{Ar} \mathrm{H}) ; \delta_{\mathrm{c}}\left(100 \mathrm{MHz}, \mathrm{CDCl}_{3}\right) 35.3\left(\mathrm{NCH}_{3}\right), 108.1(\mathrm{ArCH}), 109.2(\mathrm{ArCH}), 122.1(\mathrm{ArCH})$, 123.1 ( $\mathrm{ArCH}), 124.2(\mathrm{ArCH}), 128.8(\mathrm{ArCH}), 129.6(\mathrm{ArCH}), 130.3(\mathrm{ArCH}), 130.6(\mathrm{ArCH}), 130.9(\operatorname{ArC})$, $131.1(\operatorname{ArC}), 132.4(\operatorname{ArC}), 133.1(\operatorname{Ar} C), 133.6(\operatorname{ArC}), 134.8(\operatorname{Ar} C), 136.2(\operatorname{ArC}), 140.4(\operatorname{ArCH}), 146.8$ $(\operatorname{ArC}), 151.5(\operatorname{ArCH}), 162.6(C=0) ; v_{\max }\left(\right.$ thin film $\left./ \mathrm{cm}^{-1}\right)$ 733, 831, 900, 1013, 1066, 1089, 1150, 1286, $1308,1398,1474,1513,1580,1667,2923,3047,3248,3390 ;$ MS (GCMS) m/z $421.1\left(M^{+}\right)$; HRMS $\mathrm{C}_{23} \mathrm{H}_{18} \mathrm{ON}_{3} \mathrm{Cl}_{2}^{+}\left(\mathrm{M}+\mathrm{H}^{+}\right)$Expected 422.0821, Found 422.0821.

53 was further characterized by X-ray crystallographic analysis. CCDC: 1922368.

Isopropyl 2-(4-(4-chlorobenzoyl)-2-(1-methyl-1H-pyrrol-2-yl)phenoxy)-2-methylpropanoate, 54

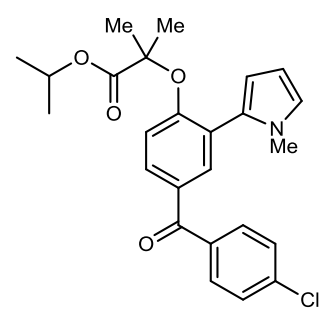

Prepared as described in General Procedure C: fenofibrate $(72 \mathrm{mg}, 0.20 \mathrm{mmol})$, dibenzothiophene $\mathrm{S}$ oxide (44 mg, $0.22 \mathrm{mmol}), \mathrm{Tf}_{2} \mathrm{O}(41 \mu \mathrm{L}, 0.24 \mathrm{mmol}), \mathrm{PTH}(5.5 \mathrm{mg}, 0.02 \mathrm{mmol}), \mathrm{N}$-methylpyrrole $(0.36$ $\mathrm{mL}, 4.00 \mathrm{mmol}, 20$ equiv.), after purification by column chromatography on silica gel (10\% EtOAc in hexanes), gave $54(49.2 \mathrm{mg}, 0.11 \mathrm{mmol}, 56 \%)$ as a white solid; m.p. (recrystallized from $\left.\mathrm{CHCl}_{3}\right) 99-$ $101{ }^{\circ} \mathrm{C} ; \delta_{\mathrm{H}}\left(400 \mathrm{MHz}, \mathrm{CDCl}_{3}\right) 1.21\left(\mathrm{~d}, J=6.3 \mathrm{~Hz}, 6 \mathrm{H}, \mathrm{CH}_{3}\right), 1.55\left(\mathrm{~s}, 6 \mathrm{H}, \mathrm{CH}_{3}\right), 3.58\left(\mathrm{~s}, 3 \mathrm{H}, \mathrm{NCH}_{3}\right), 5.08$ (hept, $J=6.3 \mathrm{~Hz}, 1 \mathrm{H}$, Alk CH), $6.13(\mathrm{dd}, J=3.5,1.9 \mathrm{~Hz}, 1 \mathrm{H}, \operatorname{Ar} H$ ), 6.19 (dd, $J=3.5,2.6 \mathrm{~Hz}, 1 \mathrm{H}, \operatorname{Ar} H$ ), $6.73(\mathrm{dd}, J=2.6,1.9 \mathrm{~Hz}, 1 \mathrm{H}, \operatorname{Ar} H), 6.84(\mathrm{~d}, J=8.5 \mathrm{~Hz}, 1 \mathrm{H}, \operatorname{Ar} H), 7.45(\mathrm{~d}, J=8.5 \mathrm{~Hz}, 2 \mathrm{H}, \operatorname{Ar} H), 7.68-$ $7.79(\mathrm{~m}, 4 \mathrm{H}, \mathrm{Ar} \mathrm{H}) ; \delta_{\mathrm{C}}\left(100 \mathrm{MHz}, \mathrm{CDCl}_{3}\right) 21.7\left(\mathrm{CH}_{3}\right), 25.3\left(\mathrm{CH}_{3}\right), 35.0\left(\mathrm{NCH}_{3}\right), 69.5(\mathrm{Alk} \mathrm{CH}), 80.2\left(\mathrm{CH}_{3} \mathrm{C}\right)$, 107.6 ( $\mathrm{ArCH}), 109.7(\operatorname{ArCH}), 115.7(\operatorname{ArCH}), 123.0(\operatorname{ArCH}), 125.1(\operatorname{ArC}), 128.7(\operatorname{ArCH}), 130.4(\operatorname{ArC})$, 130.5 (ArC), $130.9(\operatorname{ArCH}), 131.3(\operatorname{ArCH}), 135.1(\operatorname{ArCH}), 136.4(\operatorname{ArC}), 138.6(\operatorname{ArC} C), 157.7(\operatorname{ArC} C), 173.2$ $(C=0), 194.3(C=0) ; v_{\max }\left(\right.$ thin film/ $\left./ \mathrm{cm}^{-1}\right) 763,847,941,1014,1089,1100,1137,1263,1384,1466$, $1495,1593,1653,1730,2938,2981 ; \mathrm{MS}(\mathrm{GCMS}) \mathrm{m} / \mathrm{z} 439.2\left(\mathrm{M}^{+}\right) ; \operatorname{HRMS} \mathrm{C}_{25} \mathrm{H}_{27} \mathrm{O}_{4} \mathrm{NCl}^{+}\left(\mathrm{M}+\mathrm{H}^{+}\right)$ Expected 440.1623, Found 440.1621. 
Ethyl 2-(4-chloro-2-(1-methyl-1H-pyrrol-2-yl)phenoxy)-2-methylpropanoate, 55<smiles></smiles>

Prepared as described in General Procedure C: clofibrate $(43 \mu \mathrm{L}, 0.20 \mathrm{mmol})$, dibenzothiophene $S$ oxide (44 mg, $0.22 \mathrm{mmol}$ ), Tf $2 \mathrm{O}$ (41 $\mu \mathrm{L}, 0.24 \mathrm{mmol}$ ), PTH (5.5 mg, $0.02 \mathrm{mmol}), \mathrm{N}$-methylpyrrole (0.36 $\mathrm{mL}, 4.00 \mathrm{mmol}, 20$ equiv.), after purification by column chromatography on silica gel $\left(\mathrm{CHCl}_{3}\right)$, gave 55 (28.3 mg, $0.09 \mathrm{mmol}, 44 \%$ ) as a colourless oil; $\delta_{\mathrm{H}}\left(400 \mathrm{MHz}, \mathrm{CDCl}_{3}\right) 1.26\left(\mathrm{t}, J=7.1 \mathrm{~Hz}, 4 \mathrm{H}, \mathrm{CH}_{3}\right), 1.34$ $\left(\mathrm{s}, 6 \mathrm{H}, \mathrm{CH}_{3}\right), 3.55\left(\mathrm{~s}, 3 \mathrm{H}, \mathrm{NCH}_{3}\right), 4.22\left(\mathrm{q}, J=7.1 \mathrm{~Hz}, 2 \mathrm{H}, \mathrm{CH}_{2}\right), 6.11$ (dd, J=3.6, 1.8 Hz, 1H, Ar H), 6.18 (dd, $J=3.6,2.7 \mathrm{~Hz}, 1 \mathrm{H}, \operatorname{Ar} H), 6.71(\mathrm{dd}, J=2.7,1.8 \mathrm{~Hz}, 1 \mathrm{H}, \operatorname{Ar} H), 6.88(\mathrm{~d}, J=8.7 \mathrm{~Hz}, 1 \mathrm{H}, \operatorname{Ar} H), 7.19$ (dd, $J=8.7,2.8 \mathrm{~Hz}, 1 \mathrm{H}, \operatorname{Ar} H), 7.29(\mathrm{~d}, J=2.8 \mathrm{~Hz}, 1 \mathrm{H}, \mathrm{Ar} \mathrm{H}) ; \delta_{\mathrm{C}}\left(100 \mathrm{MHz}, \mathrm{CDCl}_{3}\right) 14.2\left(\mathrm{CH}_{3}\right), 25.0\left(\mathrm{CH}_{3}\right)$, 35.0 $\left(\mathrm{NCH}_{3}\right), 61.6\left(\mathrm{CH}_{2}\right), 80.7\left(\mathrm{CH}_{3} \mathrm{C}\right), 107.6(\mathrm{ArCH}), 109.8(\operatorname{ArCH}), 120.5(\operatorname{ArCH}), 123.0(\operatorname{ArCH}), 127.6$ $(\operatorname{ArC}), 128.3(\operatorname{ArCH}), 128.7(\operatorname{ArC}), 130.5(\operatorname{ArC} C), 132.1(\operatorname{ArCH}), 152.2(\operatorname{ArC}), 174.2(C=0) ; v_{\max }(\operatorname{thin}$ $\mathrm{film} / \mathrm{cm}^{-1}$ ) 710, 886, 964, 1021, 1091, 1133, 1174, 1281, 1382, 1437, 1466, 1594, 1732, 2871, 2936, 2981; MS (GCMS) $\mathrm{m} / z$ 321.1 $\left(\mathrm{M}^{+}\right)$; HRMS $\mathrm{C}_{17} \mathrm{H}_{20} \mathrm{O}_{3} \mathrm{NClNa}^{+}\left(\mathrm{M}+\mathrm{Na}^{+}\right)$Expected 344.1024, Found 344.1015.

N-(1-(2,6-Dimethyl-4-(1-methyl-1H-pyrrol-2-yl)phenoxy)propan-2-yl)acetamide, 56<smiles>CC(=O)NC(C)COc1c(C)cc(-c2ccc[nH]2)cc1C</smiles>

Prepared as described in General Procedure C: $\mathbf{S 1 0}(44 \mathrm{mg}, 0.20 \mathrm{mmol})$, dibenzothiophene S-oxide (44 mg, $0.22 \mathrm{mmol}$ ), Tf $2 \mathrm{O}(41 \mu \mathrm{L}, 0.24 \mathrm{mmol}), \mathrm{PTH}(5.5 \mathrm{mg}, 0.02 \mathrm{mmol}), \mathrm{N}$-methylpyrrole $(0.36 \mathrm{~mL}$, $4.00 \mathrm{mmol}, 20$ equiv.), after purification by column chromatography on silica gel (65\% EtOAc in hexanes), gave $56(35.6 \mathrm{mg}, 0.12 \mathrm{mmol}, 59 \%)$ as a colourless oil; $\delta_{\mathrm{H}}\left(400 \mathrm{MHz}, \mathrm{CDCl}_{3}\right) 1.42(\mathrm{~d}, J=6.9$ $\left.\mathrm{Hz}, 3 \mathrm{H}, \mathrm{CH}_{3}\right), 2.04\left(\mathrm{~s}, 3 \mathrm{H}, \mathrm{CH}_{3}\right), 2.29\left(\mathrm{~s}, 6 \mathrm{H}, \mathrm{CH}_{3}\right), 3.63\left(\mathrm{~s}, 3 \mathrm{H}, \mathrm{NCH}_{3}\right), 3.75$ (dd, J = 9.1, 3.7 Hz, 1H, $\mathrm{CH}_{2}$ ), $3.84\left(\mathrm{dd}, J=9.1,3.7 \mathrm{~Hz}, 1 \mathrm{H}, \mathrm{CH}_{2}\right), 4.31-4.42(\mathrm{~m}, 1 \mathrm{H}$, Alk CH), $5.89(\mathrm{~d}, J=8.5 \mathrm{~Hz}, 1 \mathrm{H}, \mathrm{NH}), 6.14$ (dd, $J=$ 3.6, $1.8 \mathrm{~Hz}, 1 \mathrm{H}, \operatorname{Ar} H), 6.14-6.20(\mathrm{~m}, 1 \mathrm{H}, \operatorname{Ar} H), 6.65-6.71(\mathrm{~m}, 1 \mathrm{H}, \operatorname{Ar} H), 7.03(\mathrm{~s}, 2 \mathrm{H}, \operatorname{Ar} H) ; \delta_{\mathrm{c}}(100$ $\left.\mathrm{MHz}, \mathrm{CDCl}_{3}\right) 16.4\left(\mathrm{CH}_{3}\right), 17.9\left(\mathrm{CH}_{3}\right), 23.6\left(\mathrm{CH}_{3}\right), 35.1\left(\mathrm{NCH}_{3}\right), 45.6($ Alk $\mathrm{CH}), 74.2\left(\mathrm{CH}_{2}\right), 107.7(\mathrm{ArCH})$, 108.4 (ArCH), 123.3 (Ar CH), 129.4 (Ar C), 129.5 (Ar CH), 130.7 (Ar C), 134.4 (Ar C), $154.1(\operatorname{ArC} C), 169.5$ $(C=O) ; v_{\max }\left(\right.$ thin film/ $\left./ \mathrm{cm}^{-1}\right) 710,880,981,1014,1029,1088,1173,1210,1228,1275,1371,1475$, 
1548, 1644, 2873, 2924, 2971, 3078, 3284; MS (GCMS) m/z $300.1\left(\mathrm{M}^{+}\right)$; $\mathrm{HRMS} \mathrm{C}_{18} \mathrm{H}_{25} \mathrm{O}_{2} \mathrm{~N}_{2}{ }^{+}\left(\mathrm{M}^{+} \mathrm{H}^{+}\right)$ Expected 301.1911, Found 301.1906. 


\section{Mechanistic Studies}

(2-(4-(tert-Butyl)phenyl)ethene-1,1-diyl)dibenzene, $4^{16}$<smiles>C(=C(c1ccccc1)c1ccccc1)c1cc[13c]cc1</smiles>

Prepared as described in General Procedure B: 2 (93 mg, 0.20 mmol), PTH (5.5 mg, 0.02 mmol), 1,1diphenylethylene $(0.18 \mathrm{~mL}, 1.0 \mathrm{mmol}, 5.0$ equiv.), after purification by column chromatography on silica gel (10\% $\mathrm{CHCl}_{3}$ in hexanes), gave $4(43.7 \mathrm{mg}, 0.14 \mathrm{mmol}, 70 \%)$ as a white solid; $\delta_{\mathrm{H}}(500 \mathrm{MHz}$, $\left.\mathrm{CDCl}_{3}\right) 1.18\left(\mathrm{~s}, 9 \mathrm{H}, \mathrm{CH}_{3}\right), 6.85-6.91(\mathrm{~m}, 3 \mathrm{H}, \mathrm{C}=\mathrm{CH} \& \mathrm{Ar} H), 7.07(\mathrm{~d}, J=8.3 \mathrm{~Hz}, 2 \mathrm{H}, \mathrm{Ar} H), 7.13-7.34$ (m, 10H, Ar H); $\delta_{\mathrm{C}}\left(125 \mathrm{MHz}, \mathrm{CDCl}_{3}\right) 31.4\left(\mathrm{CH}_{3}\right), 34.6\left(\mathrm{CH}_{3} \mathrm{C}\right), 125.1(\mathrm{ArCH}), 127.4(\mathrm{ArCH}), 127.5(\mathrm{Ar}$ $\mathrm{CH}), 127.6(\mathrm{C}=\mathrm{CH}), 128.1(\mathrm{ArCH}), 128.3(\mathrm{ArCH}), 128.9(\mathrm{ArCH}), 129.4(\mathrm{ArCH}), 130.4(\mathrm{ArCH}), 134.5(\mathrm{Ar}$ C), $140.8(C=\mathrm{CH}), 141.7(\operatorname{Ar} C), 143.7(\operatorname{Ar} C), 150.0(\operatorname{Ar} C)$.

The data is in accordance with the literature. ${ }^{16}$

5-(2-(Allyloxy)-5-(tert-butyl)phenyl)-5H-dibenzo[b, d]thiophen-5-ium trifluoromethanesulfonate, 5<smiles>C=CCOc1ccc(Br)cc1S1(c2ccccc2)c2ccccc2-c2ccccc21</smiles>

The 2-hydroxy-5-tert-butylphenyl dibenzothiophenium salt was prepared as described in General Procedure A: 4-tert-butylphenol (451 mg, $3.0 \mathrm{mmol})$, dibenzothiophene $S$-oxide (621 mg, $3.1 \mathrm{mmol}$ ), $\mathrm{Tf}_{2} \mathrm{O}(0.53 \mathrm{~mL}, 3.2 \mathrm{mmol}$ ), gave the crude salt (ca. $733 \mathrm{mg}, 1.53 \mathrm{mmol}, 51 \%$ ) as an off-white solid, which was used in the following step without further purification; $\delta_{H}\left(400 \mathrm{MHz}, \mathrm{CD}_{3} \mathrm{CN}\right) 1.13(\mathrm{~s}, 9 \mathrm{H}$, $\left.\mathrm{CH}_{3}\right), 7.05(\mathrm{~d}, J=2.4 \mathrm{~Hz}, 1 \mathrm{H}, \operatorname{Ar} H), 7.10-7.17(\mathrm{~m}, 1 \mathrm{H}, \operatorname{Ar} H), 7.61-7.68(\mathrm{~m}, 1 \mathrm{H}, \operatorname{Ar} H), 7.70-7.76(\mathrm{~m}$, $2 \mathrm{H}, \operatorname{Ar} H), 7.89-7.98(\mathrm{~m}, 2 \mathrm{H}, \operatorname{Ar} H), 8.10$ (dd, J = 7.9, $1.0 \mathrm{~Hz}, 2 \mathrm{H}, \operatorname{Ar} H), 8.31$ (dd, J = 7.9, $1.0 \mathrm{~Hz}, 2 \mathrm{H}, \mathrm{Ar}$ $H) ; \delta_{\mathrm{C}}\left(100 \mathrm{MHz}, \mathrm{CD}_{3} \mathrm{CN}\right) 31.0\left(\mathrm{CH}_{3}\right), 35.2\left(\mathrm{CH}_{3} \mathrm{C}\right), 110.8(\operatorname{ArC}), 118.5(\mathrm{ArCH}), 125.3(\mathrm{ArCH}), 126.6(\mathrm{Ar}$ $\mathrm{CH}), 128.7(\mathrm{ArCH}), 131.0(\operatorname{ArC}), 132.2(\mathrm{ArCH}), 135.0(\operatorname{ArC}), 135.5(\operatorname{ArCH}), 135.5(\operatorname{ArCH}), 146.4(\operatorname{Ar}$ C), $156.7(\mathrm{ArC}), \mathrm{CF}_{3}$ not observed; $\delta_{\mathrm{F}}\left(376 \mathrm{MHz}, \mathrm{CD}_{3} \mathrm{CN}\right)-79.3$.

Allyl bromide ( $0.13 \mathrm{~mL}, 1.40 \mathrm{mmol}, 1.1$ equiv.) was added to a stirred suspension of the crude 2hydroxyphenyl dibenzothiophenium salt ( $615 \mathrm{mg}, 1.27 \mathrm{mmol}, 1.0$ equiv.) and $\mathrm{K}_{2} \mathrm{CO}_{3}(527 \mathrm{mg}, 3.81$ 
mmol, 3.0 equiv.) in acetone $(6.4 \mathrm{~mL})$ and the resulting mixture was heated to $65{ }^{\circ} \mathrm{C}$ for 12 hours. After cooling to ambient temperature, the reaction mixture was concentrated in vacuo, diluted with $\mathrm{CH}_{2} \mathrm{Cl}_{2}(25 \mathrm{~mL})$, washed with water $(2 \times 25 \mathrm{~mL})$ and brine $(25 \mathrm{~mL})$, dried over $\mathrm{MgSO}_{4}$, filtered, and concentrated in vacuo. The crude product was purified by recrystallization (EtOAc/hexanes), to give 5 (624 mg, $1.19 \mathrm{mmol}, 94 \%$ ) as a white solid; m.p. (recrystallized from $\mathrm{CH}_{2} \mathrm{Cl}_{2} / \mathrm{Et}_{2} \mathrm{O}$ ) $163-165{ }^{\circ} \mathrm{C} ; \delta_{\mathrm{H}}$ $\left(400 \mathrm{MHz}, \mathrm{CDCl}_{3}\right) 1.21\left(\mathrm{~s}, 9 \mathrm{H}, \mathrm{CH}_{3}\right), 4.36$ (d, J = $5.8 \mathrm{~Hz}, 2 \mathrm{H}, \mathrm{CH}_{2}$ ), 5.09 (dd, J=17.1, $1.3 \mathrm{~Hz}, 1 \mathrm{H}$, $\left.\mathrm{CH}=\mathrm{CH}_{2}\right), 5.15\left(\mathrm{dd}, \mathrm{J}=10.4,1.3 \mathrm{~Hz}, 1 \mathrm{H}, \mathrm{CH}=\mathrm{CH}_{2}\right), 5.44-5.60\left(\mathrm{~m}, 1 \mathrm{H}, \mathrm{CH}=\mathrm{CH}_{2}\right), 6.97(\mathrm{~d}, J=8.8 \mathrm{~Hz}, 1 \mathrm{H}$, $\operatorname{Ar} H$ ), $7.56-7.68(\mathrm{~m}, 4 \mathrm{H}, \operatorname{Ar} H), 7.80(\mathrm{td}, J=7.7,1.1 \mathrm{~Hz}, 2 \mathrm{H}, \operatorname{Ar} H), 7.98-8.09(\mathrm{~m}, 2 \mathrm{H}, \operatorname{Ar} H), 8.19$ (dd, $J=7.8,1.1 \mathrm{~Hz}, 2 \mathrm{H}, \operatorname{Ar} H) ; \delta_{\mathrm{C}}\left(100 \mathrm{MHz}, \mathrm{CDCl}_{3}\right) 31.0\left(\mathrm{CH}_{3}\right), 34.8\left(\mathrm{CH}_{3} \mathrm{C}\right), 70.7\left(\mathrm{CH}_{2}\right), 111.0(\operatorname{ArC}), 114.6$ $(\operatorname{ArCH}), 120.0\left(\mathrm{CH}=\mathrm{CH}_{2}\right), 121.0\left(\mathrm{q}, J=320.9 \mathrm{~Hz}, \mathrm{CF}_{3}\right), 124.1(\mathrm{ArCH}), 128.0(\mathrm{ArCH}), 129.5(\mathrm{ArCH})$, 129.6 $\left(\mathrm{CH}=\mathrm{CH}_{2}\right), 130.7(\operatorname{ArCH}), 131.2(\mathrm{ArCH}), 134.1(\operatorname{ArCH}), 135.0(\operatorname{ArC}), 139.6(\operatorname{ArC}), 146.7(\operatorname{ArC})$, $155.9(\operatorname{ArC}) ; \delta_{\mathrm{F}}\left(376 \mathrm{MHz}, \mathrm{CDCl}_{3}\right)-78.2 ; v_{\max }\left(\right.$ thin film $\left./ \mathrm{cm}^{-1}\right)$ 758, 824, 939, 988, 1029, 1152, 1223, 1257, 1426, 1493, 1559, 2871, 2963, 3087; MS $\left(\mathrm{ES}^{+}\right) \mathrm{m} / \mathrm{z} 373.2\left(\mathrm{M}^{+}\right) ; \mathrm{HRMS} \mathrm{C}_{25} \mathrm{H}_{25} \mathrm{OS}^{+}\left(\mathrm{M}^{+}\right)$Expected 373.1621, Found 373.1612.

2-((5-(tert-Butyl)-2,3-dihydrobenzofuran-3-yl)methyl)-1-methyl-1H-pyrrole, 6

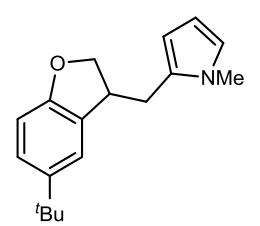

Prepared as described in General Procedure B: 5 (105 mg, 0.20 mmol), PTH (5.5 mg, 0.02 mmol), Nmethylpyrrole ( $0.36 \mathrm{~mL}, 4.00 \mathrm{mmol}, 20$ equiv.), after purification by column chromatography on silica gel (5\% EtOAc in hexanes), gave $6(11.3 \mathrm{mg}, 0.04 \mathrm{mmol}, 21 \%)$ as a colourless oil; $\delta_{H}(500 \mathrm{MHz}$, $\left.\mathrm{CDCl}_{3}\right) 1.27\left(\mathrm{~s}, 9 \mathrm{H}, \mathrm{CH}_{3}\right), 2.84\left(\mathrm{dd}, J=15.2,8.9 \mathrm{~Hz}, 1 \mathrm{H}, \mathrm{BnCH}\right.$ ), $2.92\left(\mathrm{dd}, J=15.2,6.5 \mathrm{~Hz}, 1 \mathrm{H}, \mathrm{BnCH}_{2}\right.$ ), $3.41\left(\mathrm{~s}, 3 \mathrm{H}, \mathrm{NCH}_{3}\right), 3.64-3.76(\mathrm{~m}, 1 \mathrm{H}, \mathrm{BnCH}), 4.32\left(\mathrm{dd}, J=8.9,5.2 \mathrm{~Hz}, 1 \mathrm{H}, \mathrm{OCH}_{2}\right), 4.64(\mathrm{t}, J=8.9 \mathrm{~Hz}$, $\left.1 \mathrm{H}, \mathrm{OCH} \mathrm{C}_{2}\right), 5.89-5.99(\mathrm{~m}, 1 \mathrm{H}, \operatorname{Ar} H), 6.03-6.15(\mathrm{~m}, 1 \mathrm{H}, \operatorname{Ar} H), 6.51-6.61(\mathrm{~m}, 1 \mathrm{H}, \operatorname{Ar} H), 6.73(\mathrm{~d}, J=$ $8.4 \mathrm{~Hz}, 1 \mathrm{H}, \operatorname{Ar} H), 6.93(\mathrm{~d}, J=2.1 \mathrm{~Hz}, 1 \mathrm{H}, \operatorname{Ar} H), 7.17(\mathrm{dd}, J=8.4,2.1 \mathrm{~Hz}, 1 \mathrm{H}, \operatorname{Ar} H) ; \delta_{\mathrm{C}}\left(125 \mathrm{MHz}, \mathrm{CDCl}_{3}\right)$ 31.9 $\left(\mathrm{CH}_{3}\right), 32.1(\mathrm{BnCH}), 33.8\left(\mathrm{NCH}_{3}\right), 34.5\left(\mathrm{CH}_{3} \mathrm{C}\right), 42.3(\mathrm{BnCH}), 77.4\left(\mathrm{OCH}_{2}\right), 106.9(\mathrm{ArCH}), 106.9(\mathrm{Ar}$ $\mathrm{CH}), 108.8(\mathrm{ArCH}), 121.7(\operatorname{ArCH}), 121.7(\operatorname{ArCH}), 125.4(\operatorname{ArCH}), 129.8(\operatorname{ArC}), 130.7(\operatorname{ArC}), 143.5(\operatorname{Ar}$ C), $157.7(\operatorname{ArC}) ; v_{\max }\left(\right.$ thin film/cm ${ }^{-1}$ ) 816, 966, 1117, 1232, 1262, 1297, 1362, 1466, 1489, 2823, 2952; MS (GCMS) m/z 269.1 ( $\left.\mathrm{M}^{+}\right)$; HRMS $\mathrm{C}_{18} \mathrm{H}_{24} \mathrm{ON}^{+}\left(\mathrm{M}+\mathrm{H}^{+}\right.$) Expected 270.1852, Found 270.1848. 
NMR SPECTRA

SF $-{ }^{1}$ H NMR (400 MHz, DMSO- $\left.d_{6}\right)$

管

$\stackrel{\substack{i \\ i}}{1}$
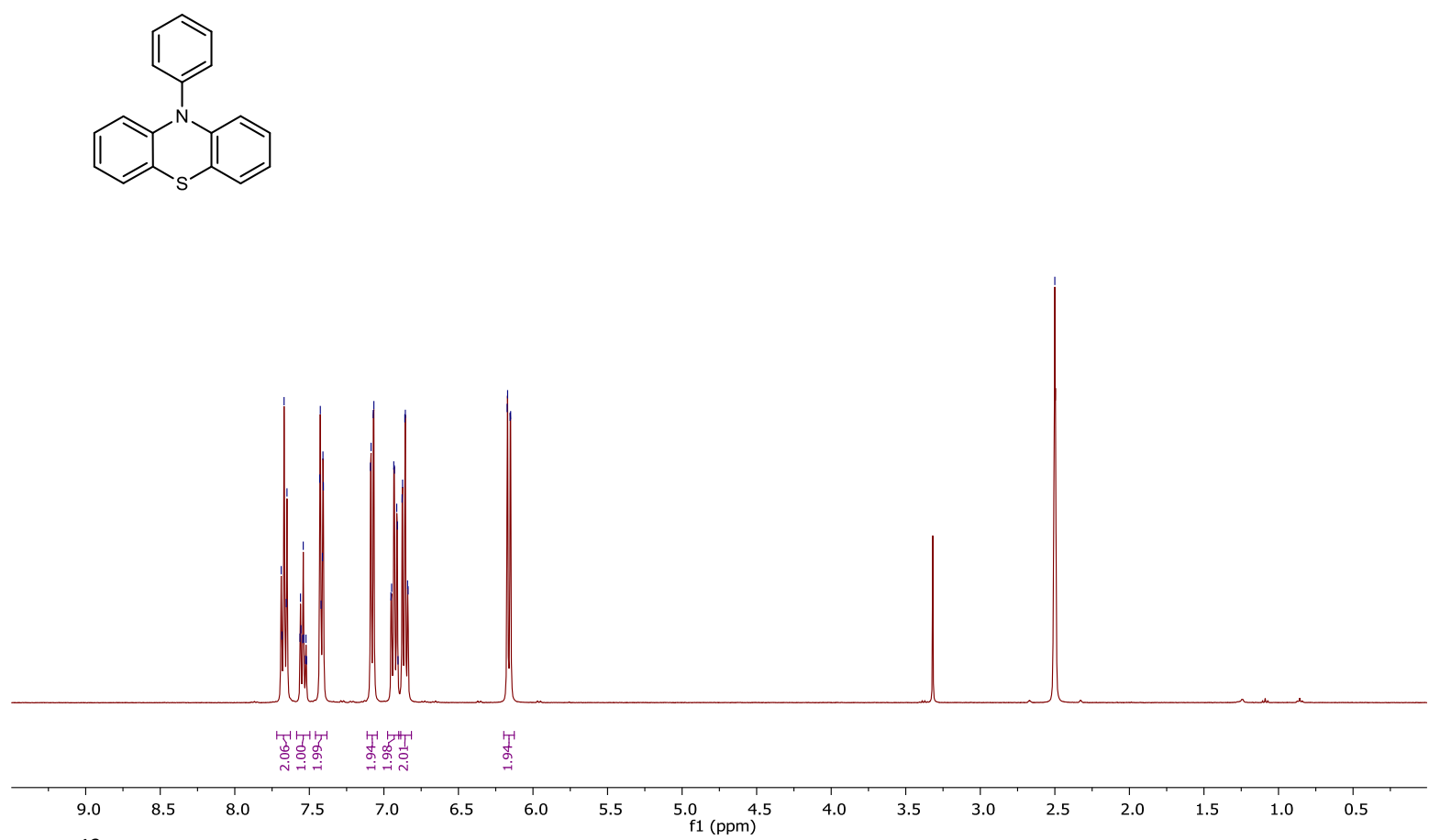

SF $-{ }^{13} \mathrm{C}$ NMR (100 MHz, DMSO- $\left.d_{6}\right)$

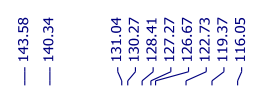
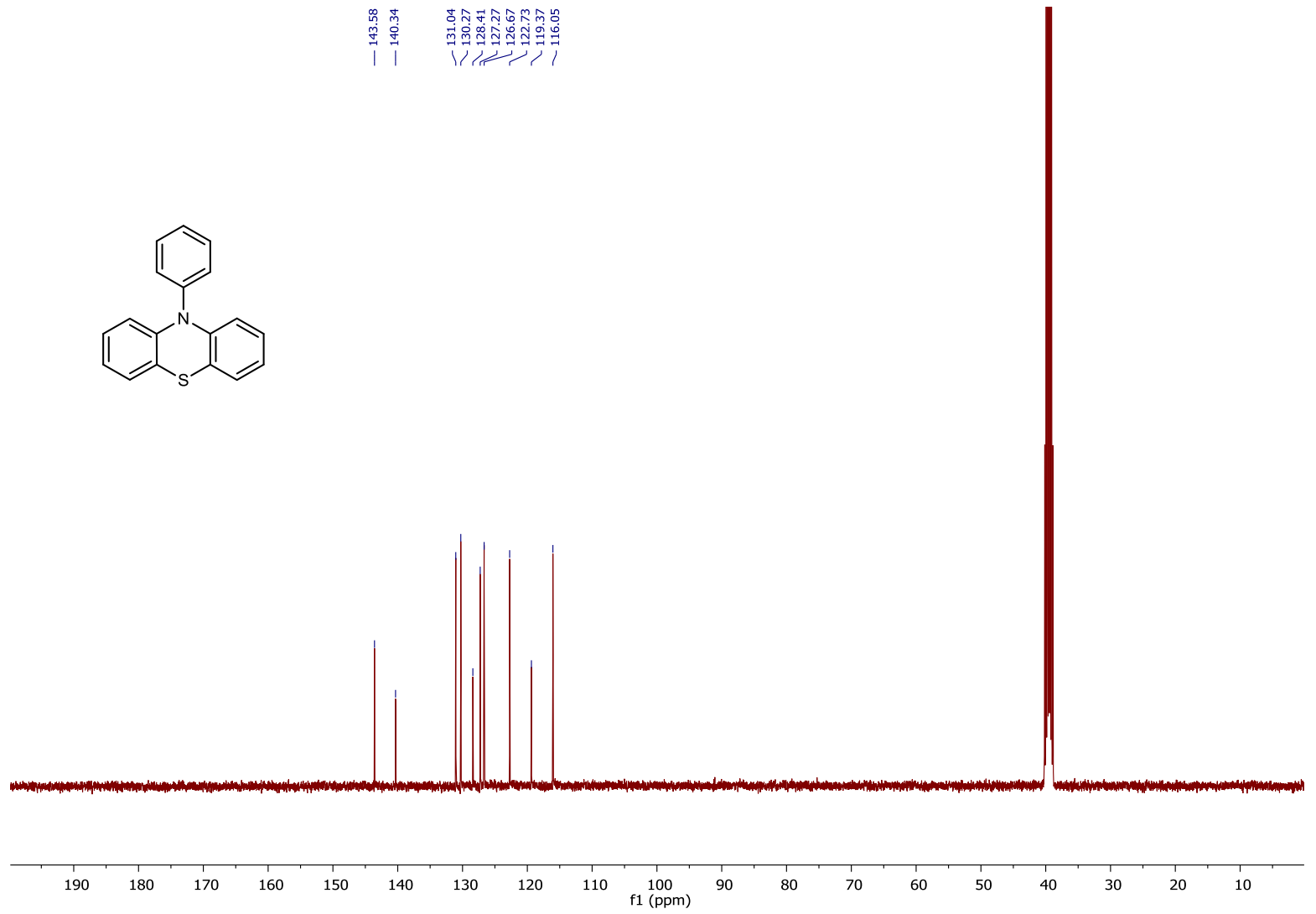

54 
S6 - ${ }^{1} \mathrm{H}$ NMR $\left(400 \mathrm{MHz}, \mathrm{CDCl}_{3}\right)$

$\underbrace{-1}$

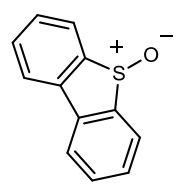

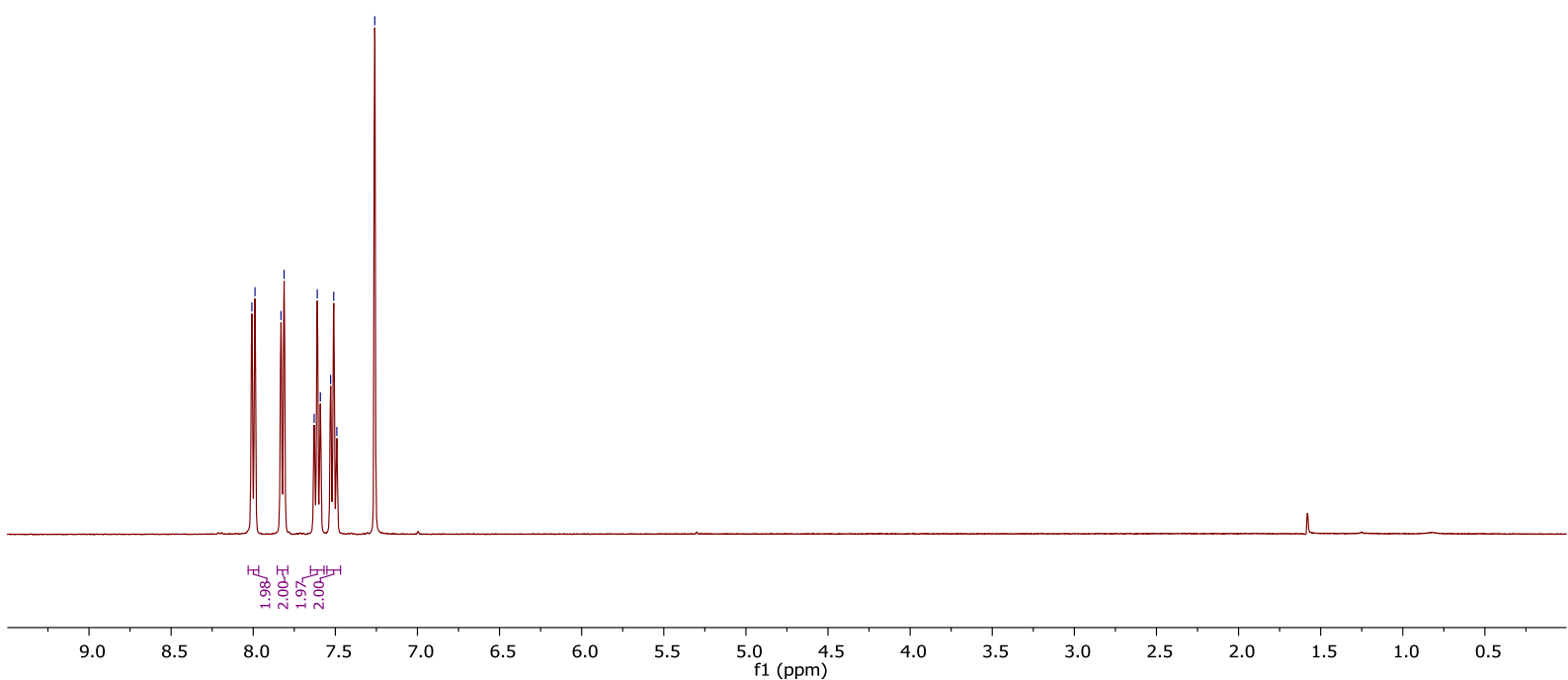

S6 $-{ }^{13} \mathrm{C} \mathrm{NMR}\left(100 \mathrm{MHz}, \mathrm{CDCl}_{3}\right)$

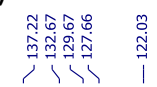

$=$

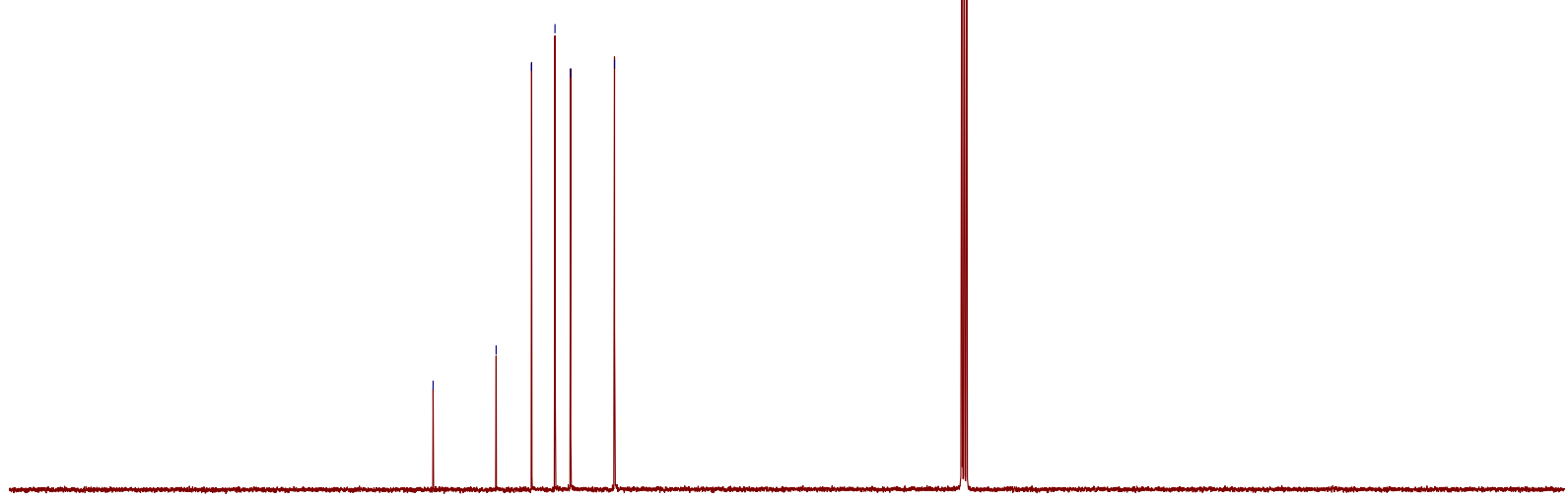

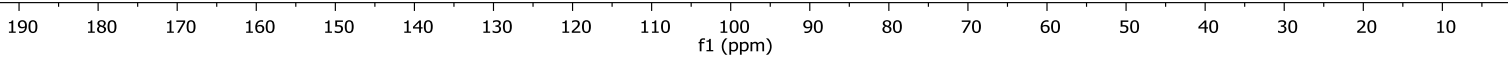


$2-{ }^{1} \mathrm{H}$ NMR $\left(400 \mathrm{MHz}, \mathrm{CDCl}_{3}\right)$

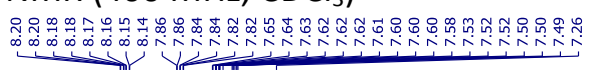

$\stackrel{\overbrace{}}{\rightarrow}$
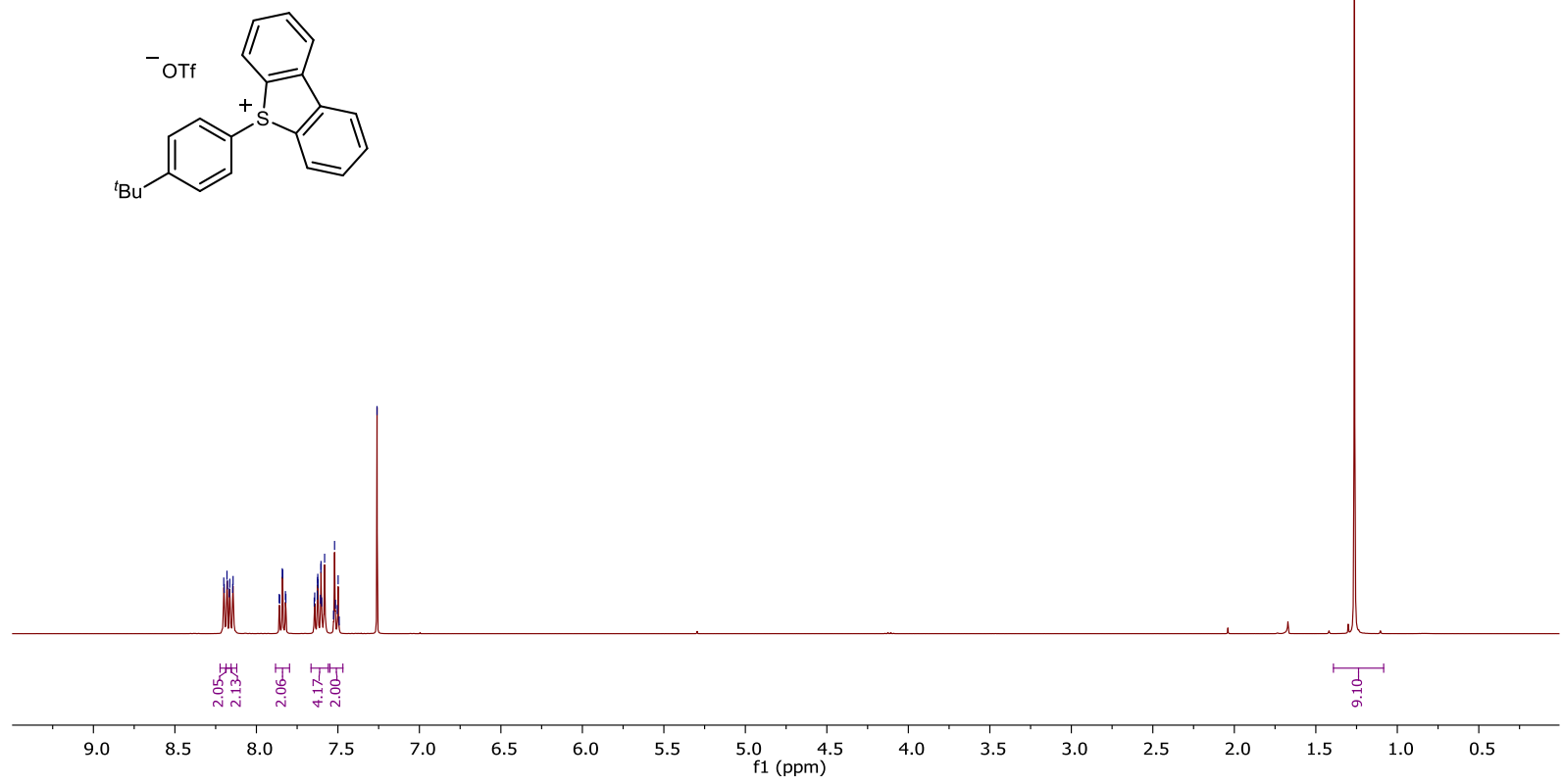

$2-{ }^{13} \mathrm{C} N M R\left(100 \mathrm{MHz}, \mathrm{CDCl}_{3}\right)$

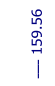

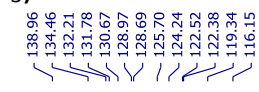

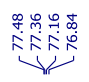

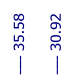
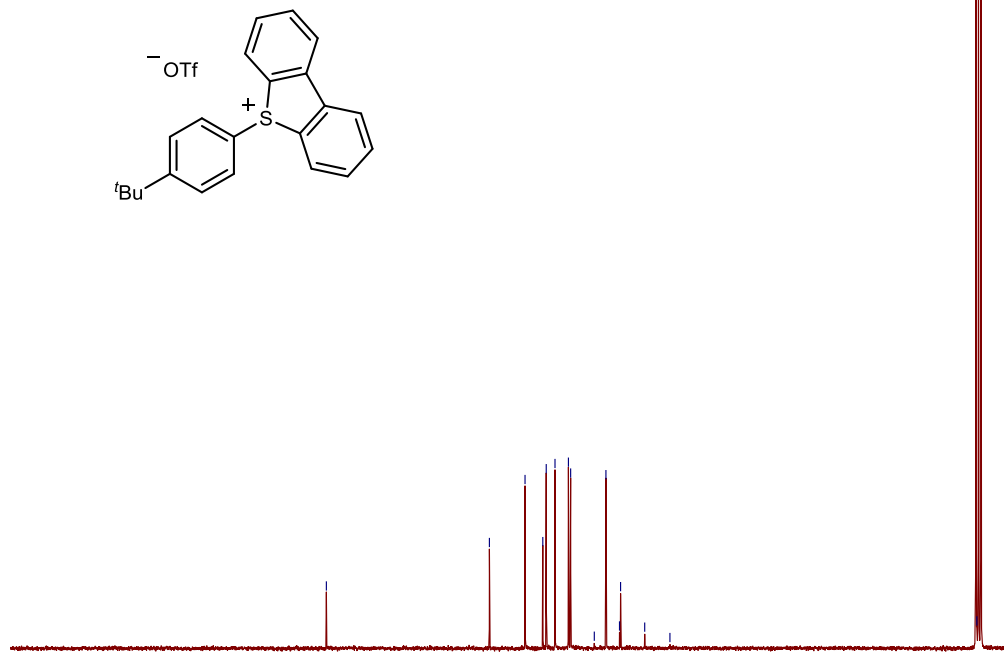

190

180

$170 \quad 160$

100
$\mathrm{f} 1(\mathrm{ppm})$

56 
$2-{ }^{19} \mathrm{~F} \mathrm{NMR}\left(376 \mathrm{MHz}, \mathrm{CDCl}_{3}\right)$

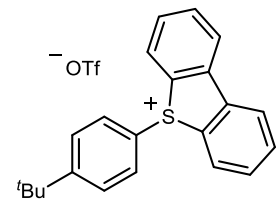

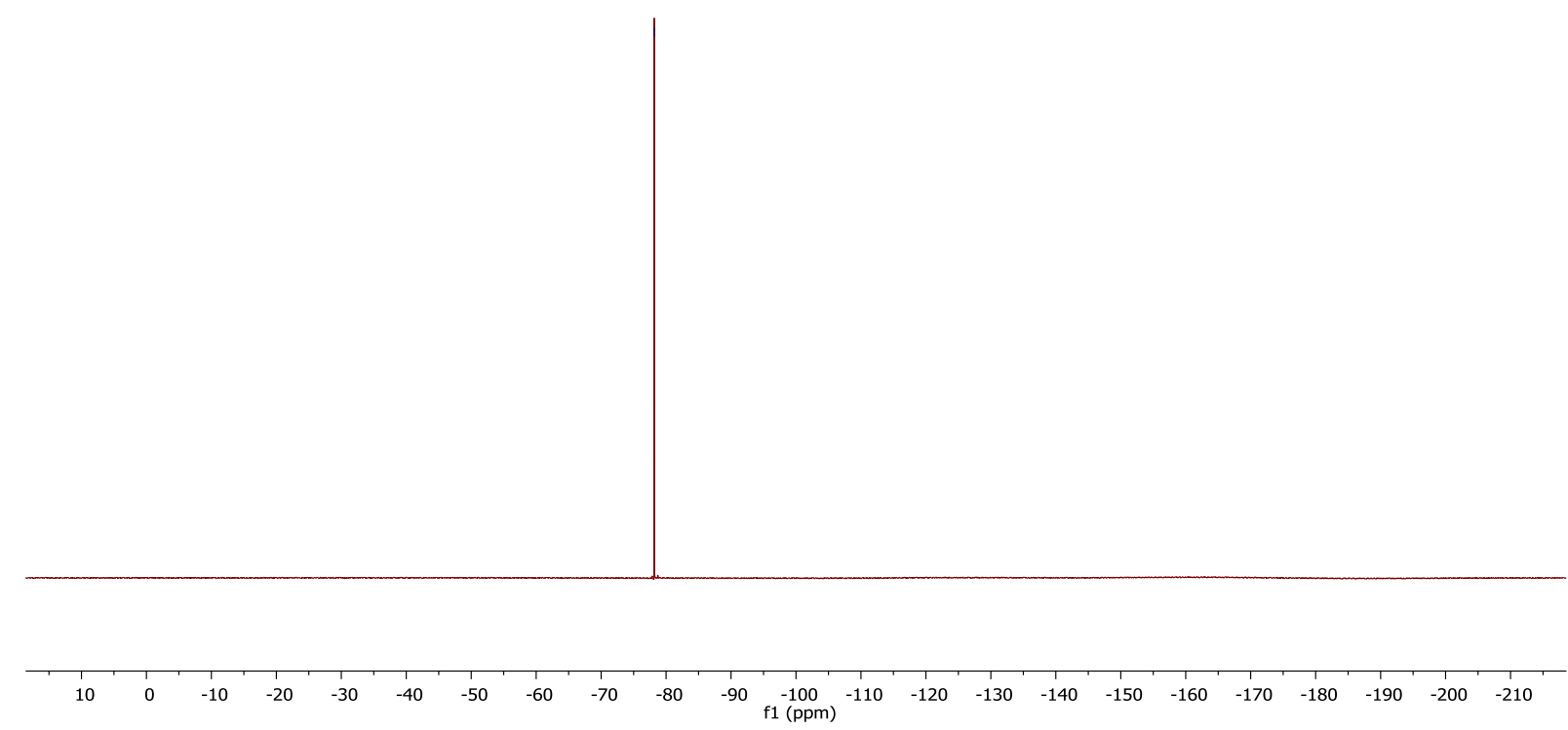


S2 $-{ }^{1} \mathrm{H}$ NMR $\left(400 \mathrm{MHz}, \mathrm{CDCl}_{3}\right)$

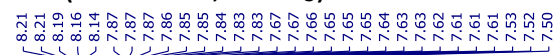
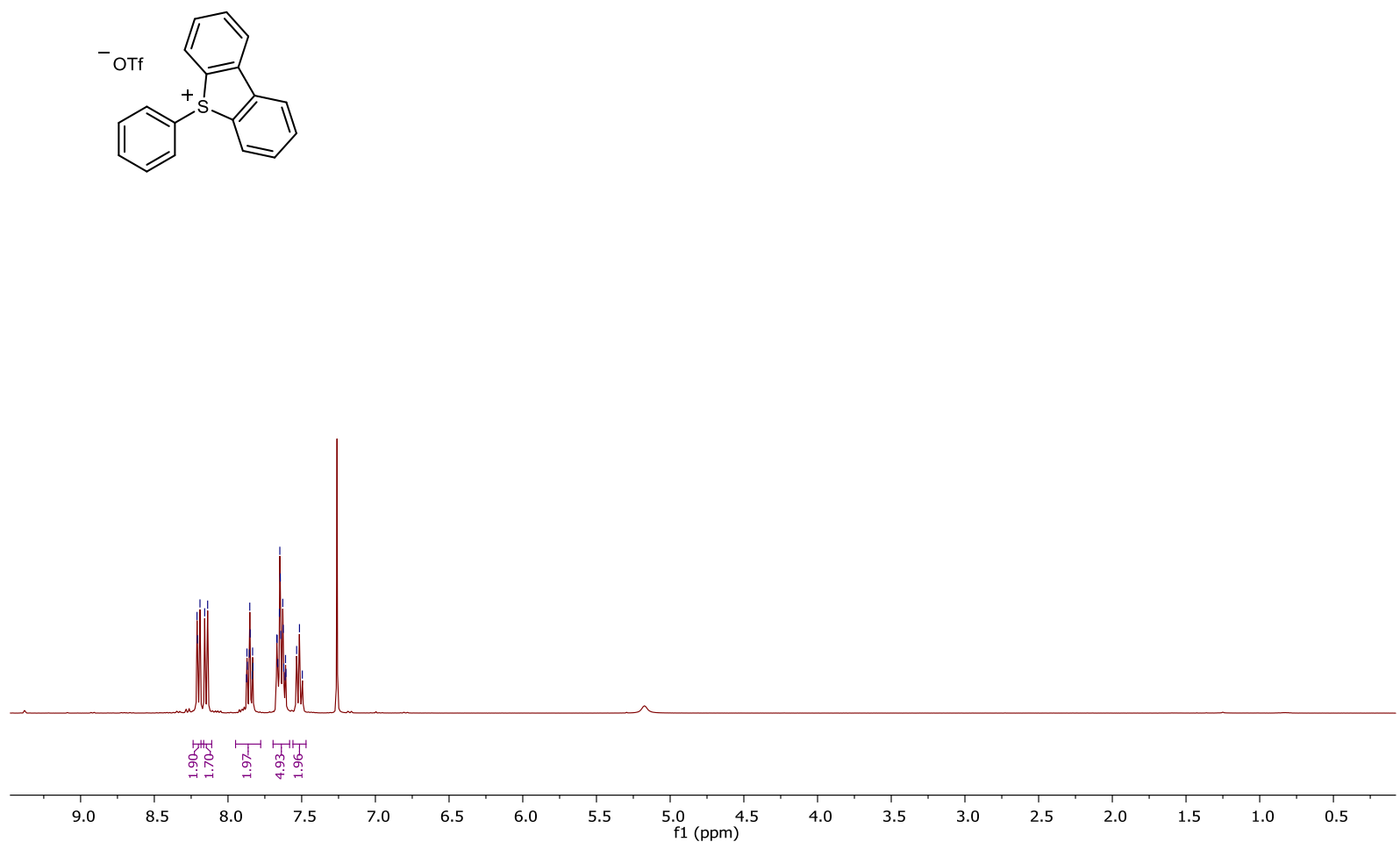

S2 $-{ }^{13} \mathrm{C} \mathrm{NMR}\left(100 \mathrm{MHz}, \mathrm{CDCl}_{3}\right)$

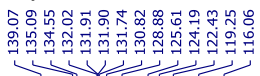
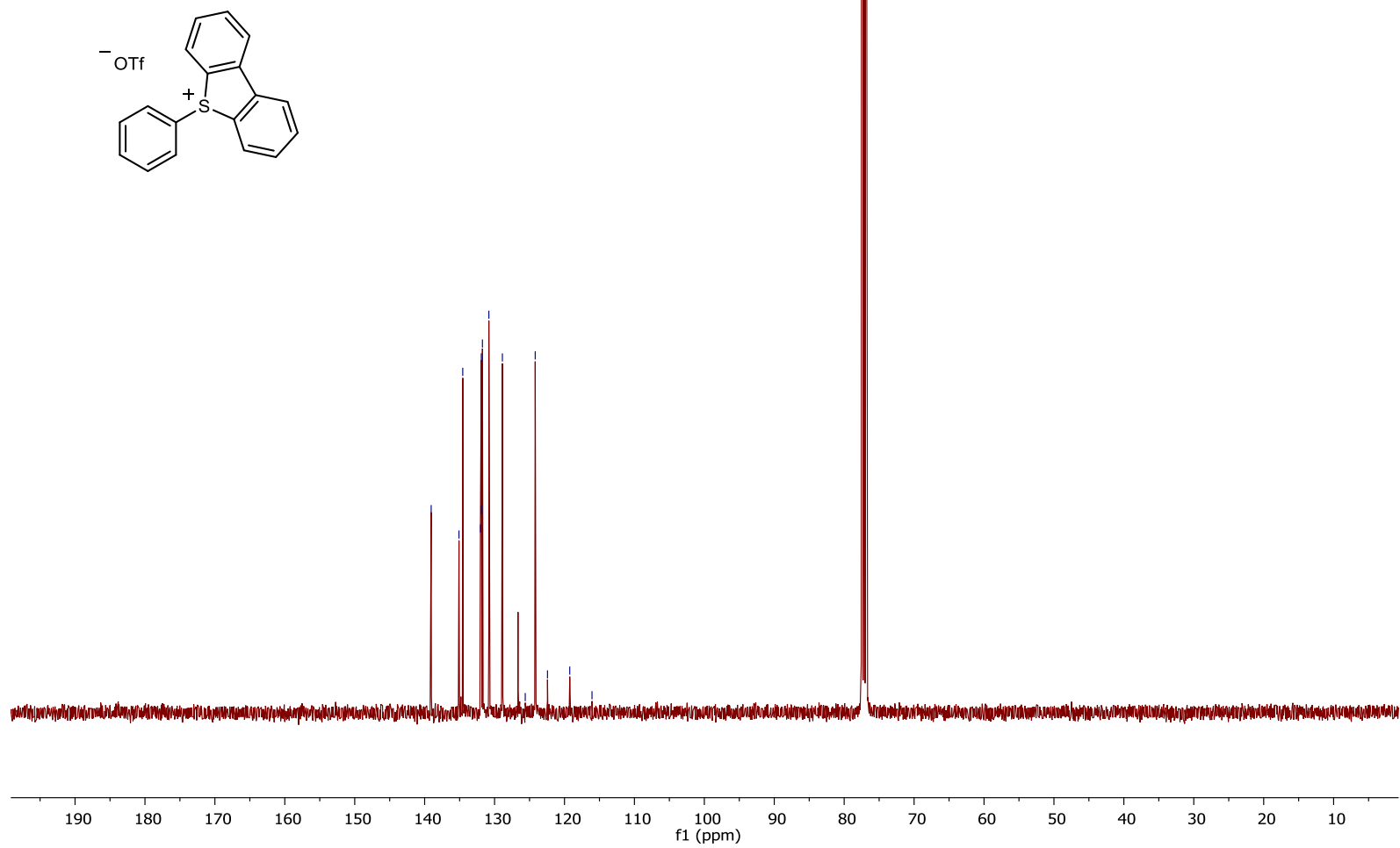

58 
S2 $-{ }^{19} \mathrm{~F}$ NMR $\left(376 \mathrm{MHz}, \mathrm{CDCl}_{3}\right)$
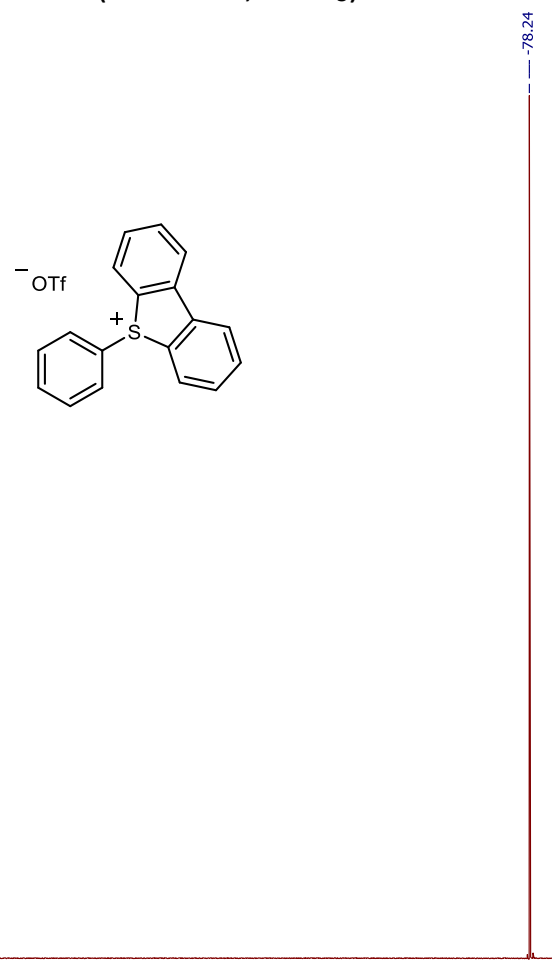

$\begin{array}{lllllllllllllllllllllllllllllll}10 & 0 & -10 & -20 & -30 & -40 & -50 & -60 & -70 & -80 & -90 & -100 & -110 & -120 & -130 & -140 & -150 & -160 & -170 & -180 & -190 & -200 & -210 & \end{array}$ 


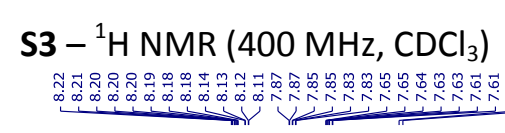

$$
-508
$$

章㘧
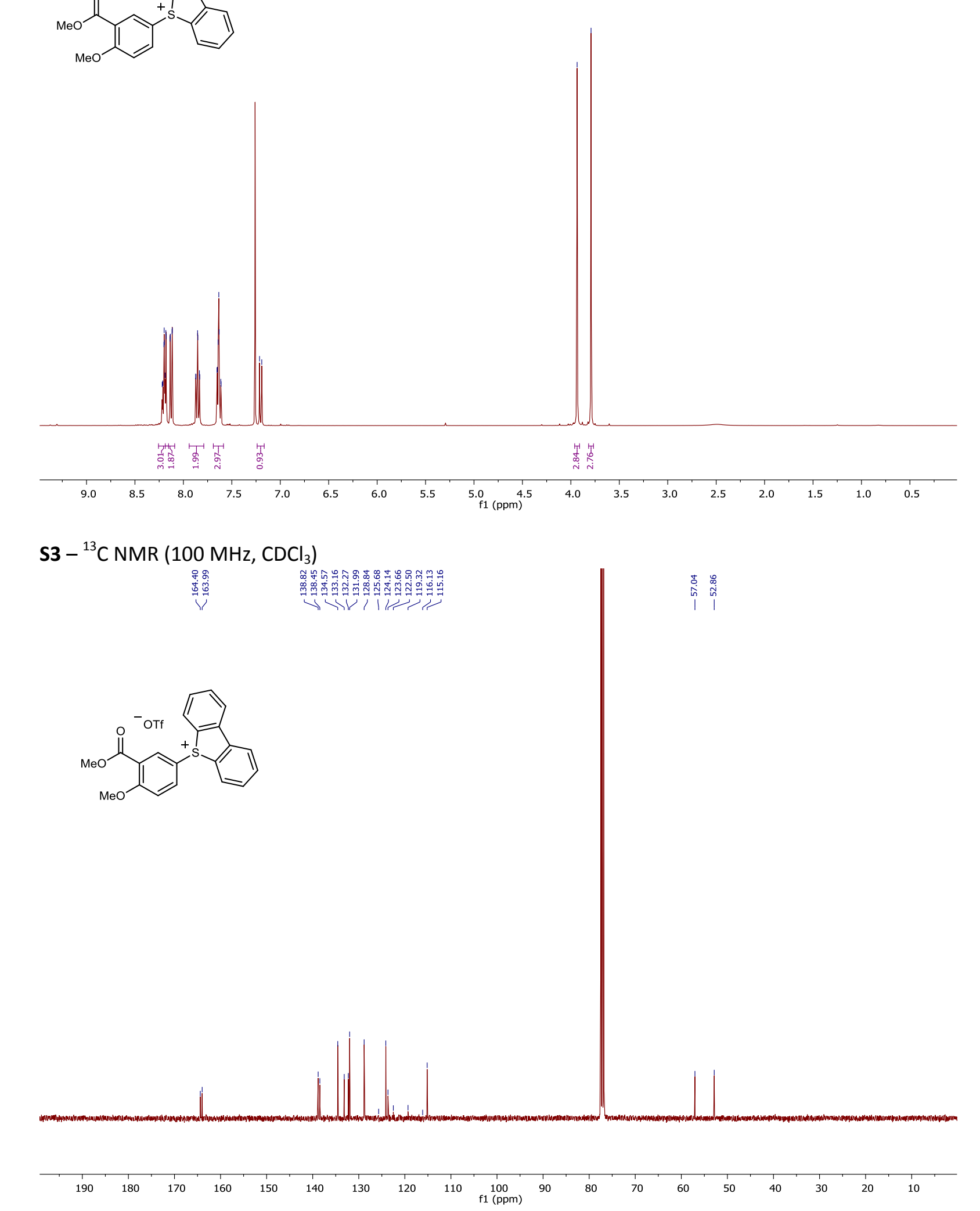
S3 $-{ }^{19} \mathrm{~F}$ NMR $\left(376 \mathrm{MHz} \mathrm{CDCl}_{3}\right)$<smiles>O=C(O[Na])c1c(S(=O)c2ccc(O)c([N+](=O)[O-])c2)ccc2c1=c1ccccc1=2</smiles> 
S4 - ${ }^{1} \mathrm{H}$ NMR $\left(400 \mathrm{MHz}, \mathrm{CDCl}_{3}\right)$

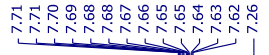<smiles></smiles>

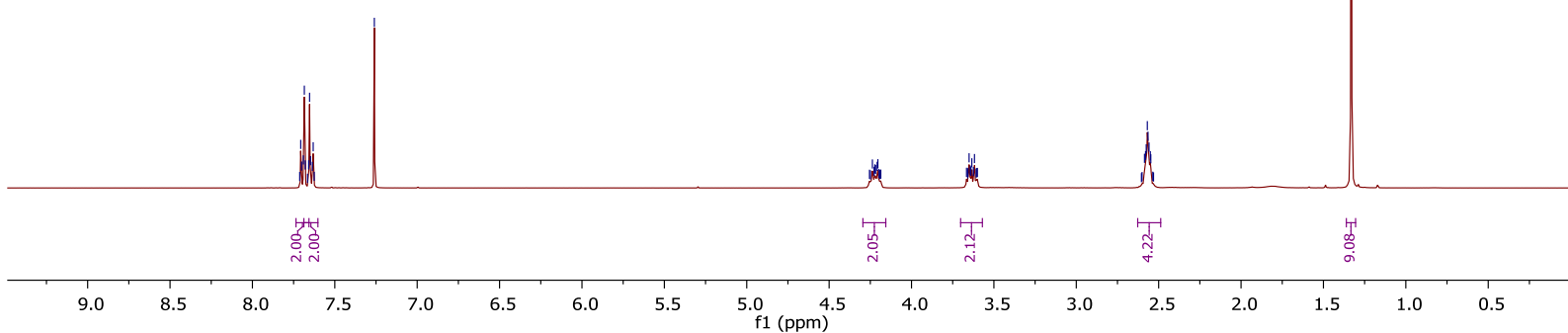

S4 $-{ }^{13} \mathrm{C}$ NMR $\left(100 \mathrm{MHz}, \mathrm{CDCl}_{3}\right)$
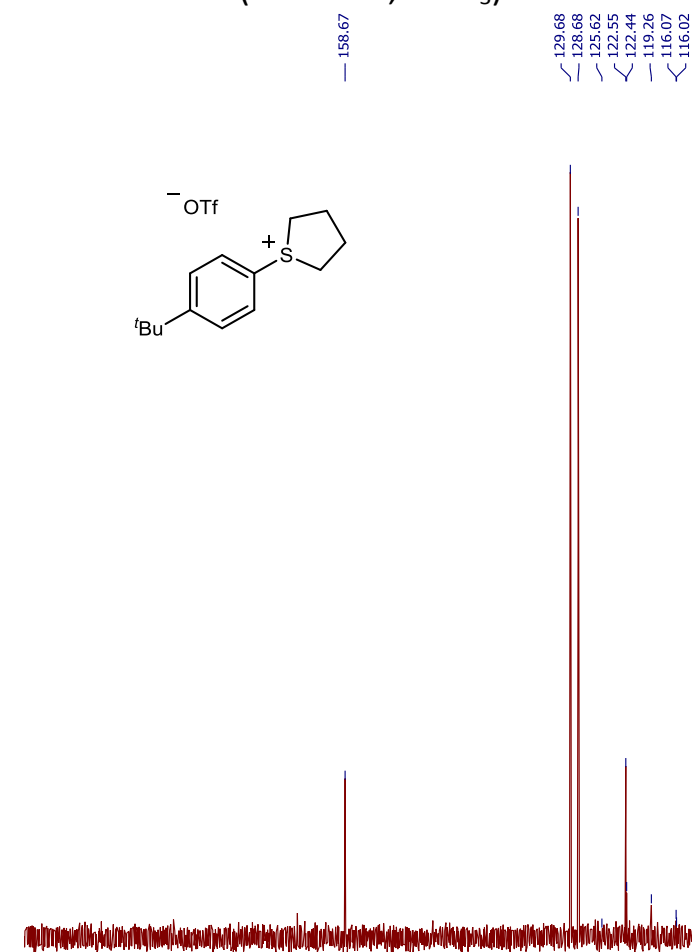

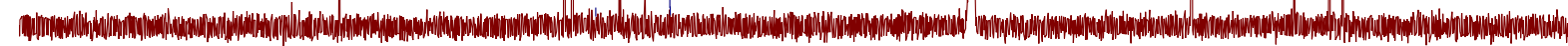

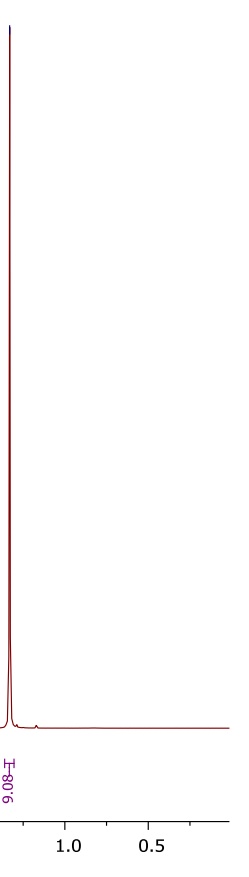


S4 $-{ }^{19}$ F NMR (376 MHz, CDCl $)$

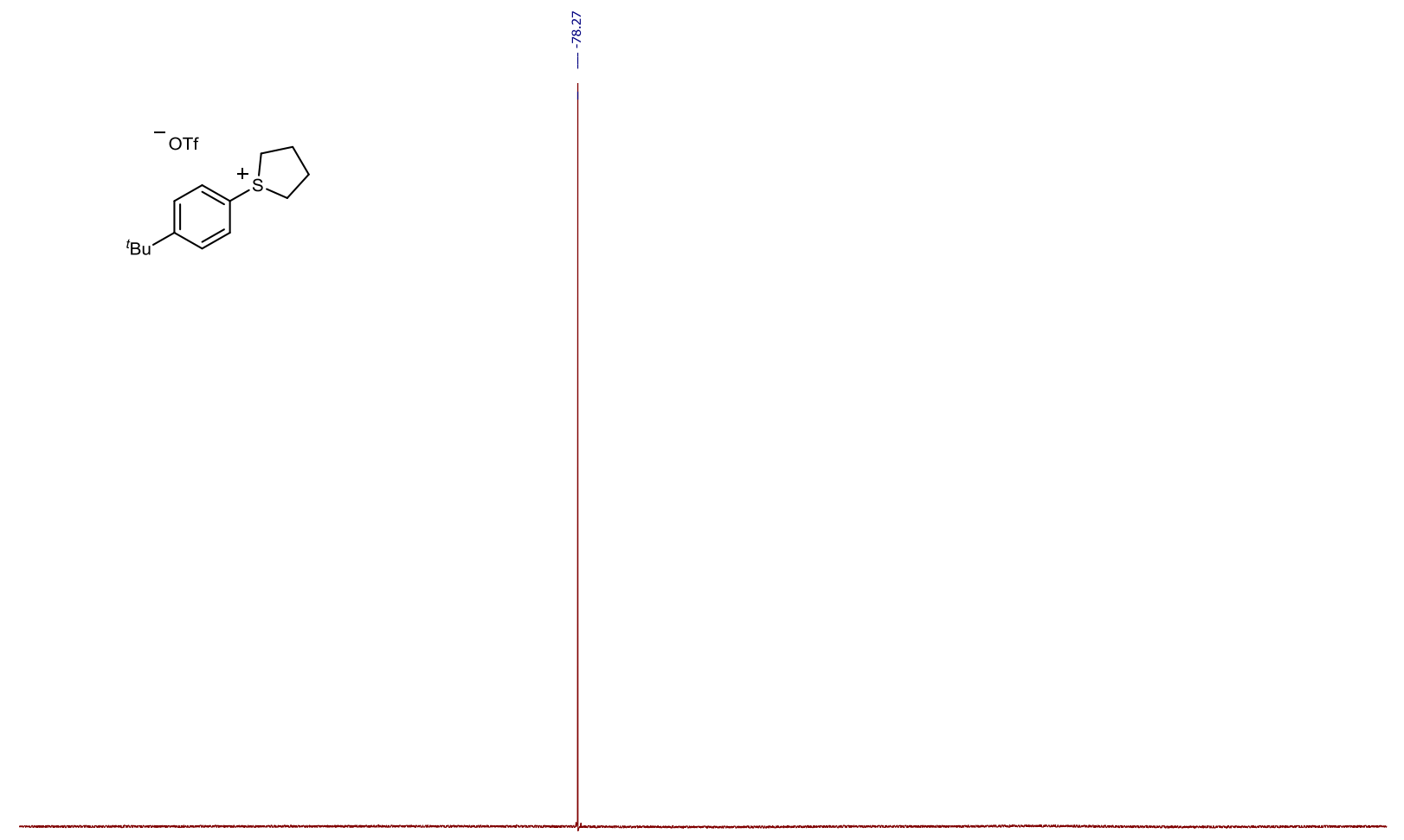

$\begin{array}{lllllllllllllllllllllllll}10 & 0 & -10 & -20 & -30 & -40 & -50 & -60 & -70 & -80 & -90 & -100 & -110 & -120 & -130 & -140 & -150 & -160 & -170 & -180 & -190 & -200 & -210 & 1\end{array}$ 


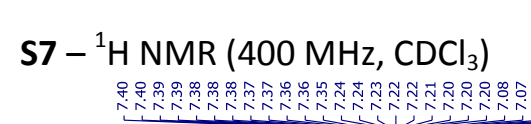

$00^{\circ} 0^{\circ}$

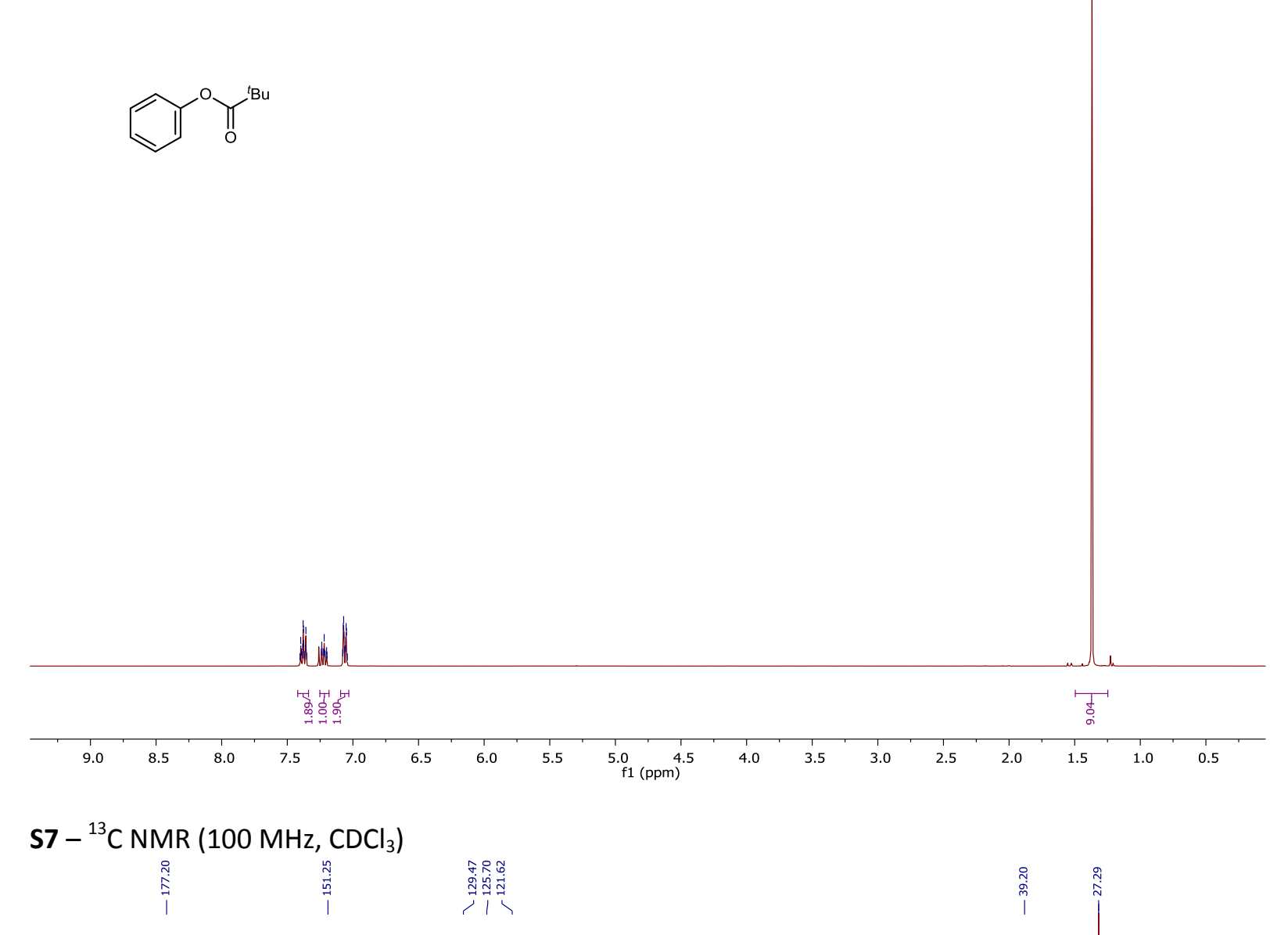

50

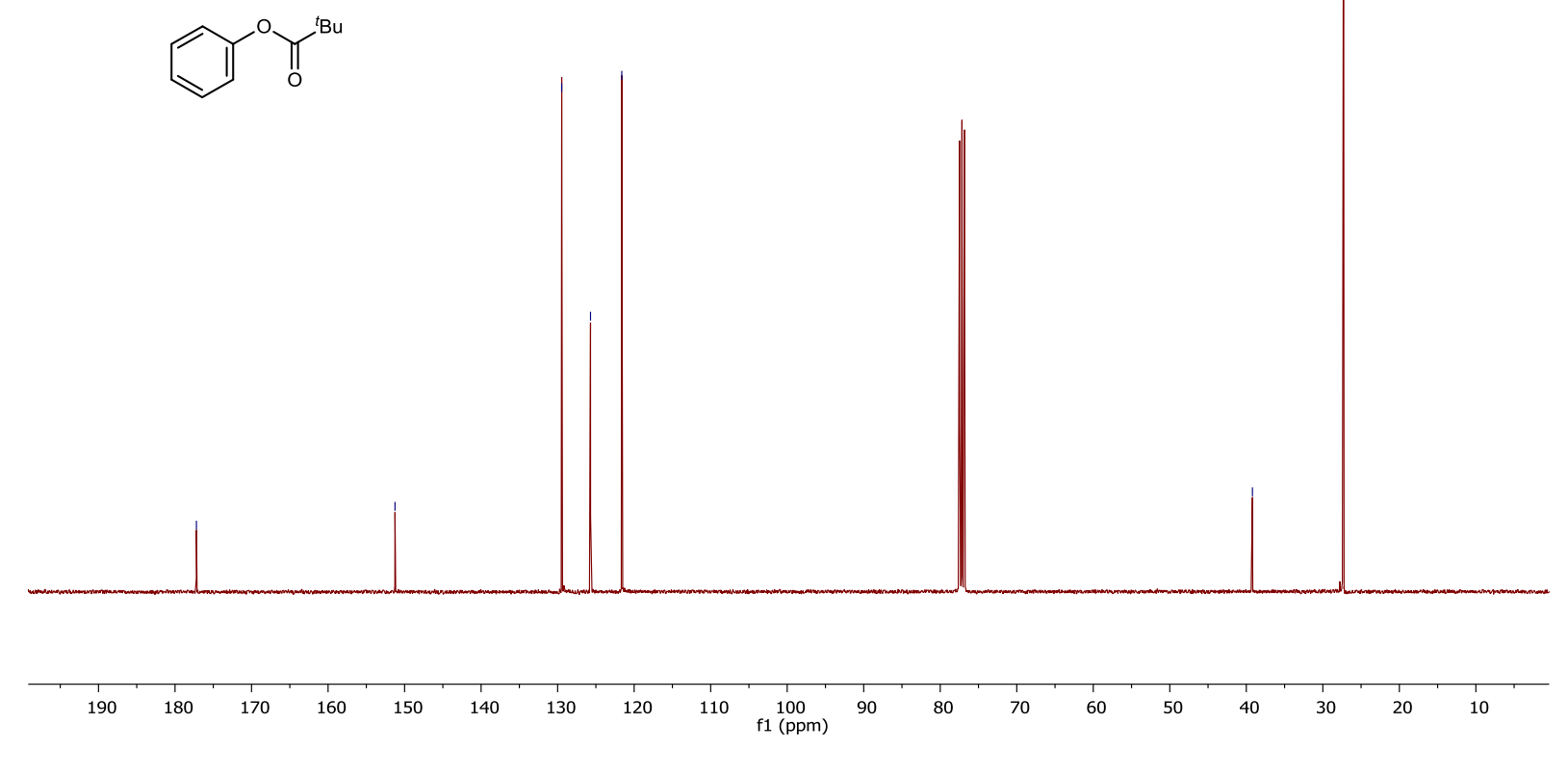


So - ${ }^{1} \mathrm{H}$ NMR $\left(400 \mathrm{MHz}, \mathrm{CDCl}_{3}\right)$

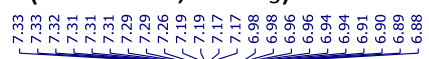

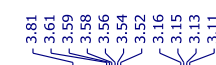

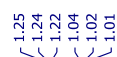
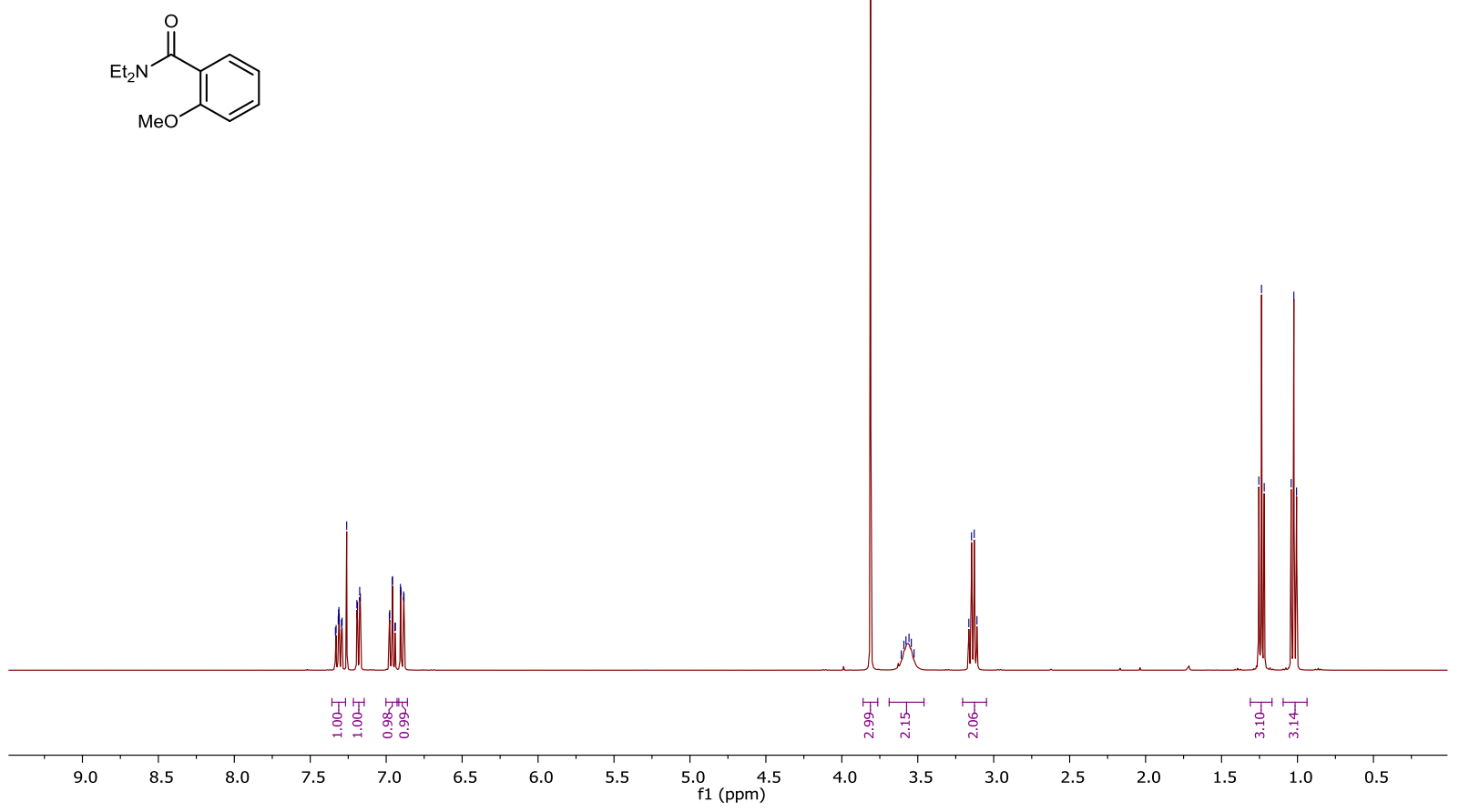

So $-{ }^{13} \mathrm{C}$ NMR $\left(100 \mathrm{MHz}, \mathrm{CDCl}_{3}\right)$

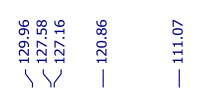

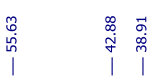

量产尊
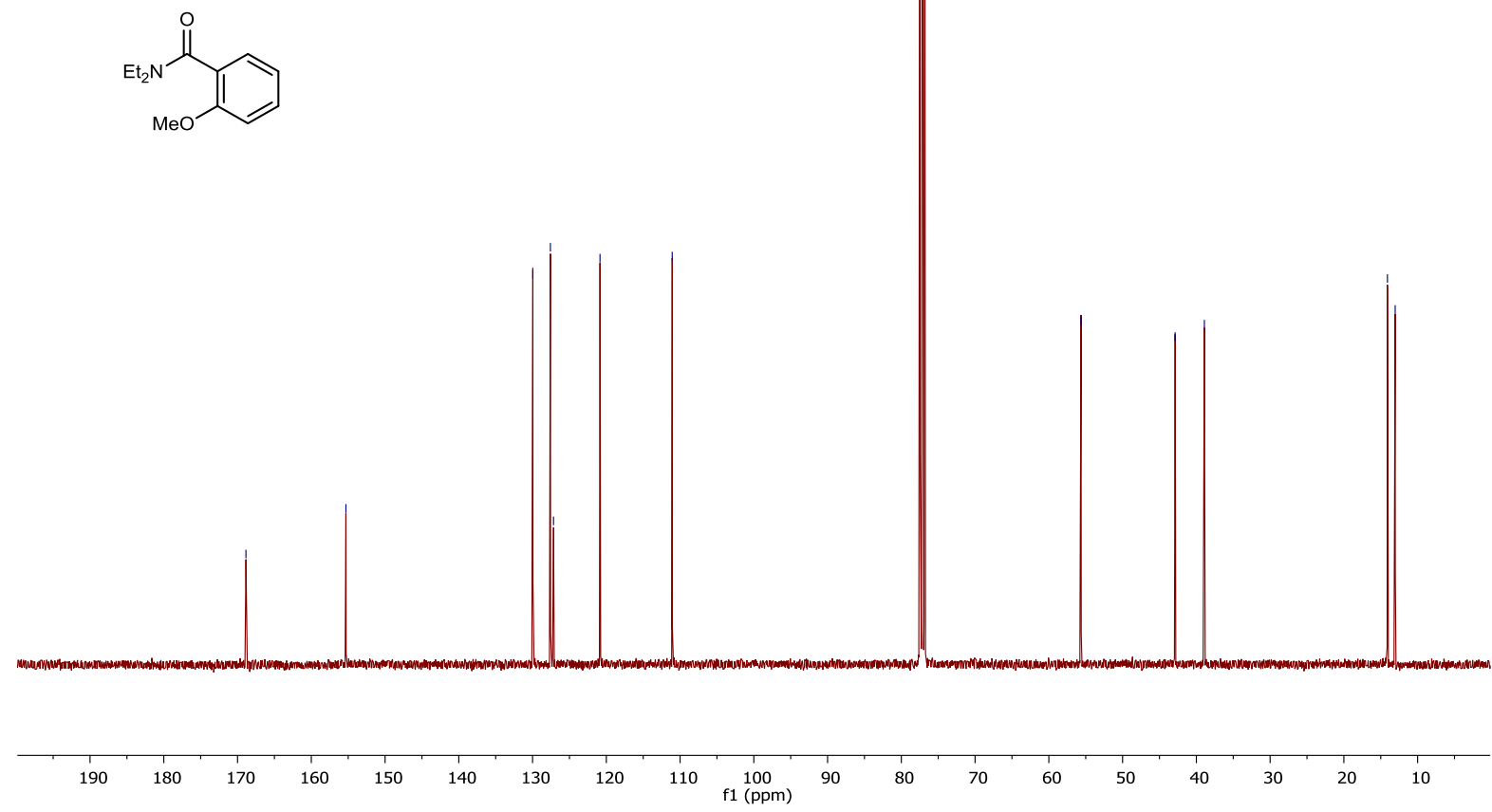

65 
S9 $-{ }^{1} \mathrm{H} \mathrm{NMR}\left(400 \mathrm{MHz}, \mathrm{CDCl}_{3}\right)$

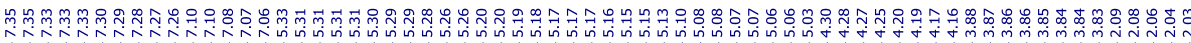
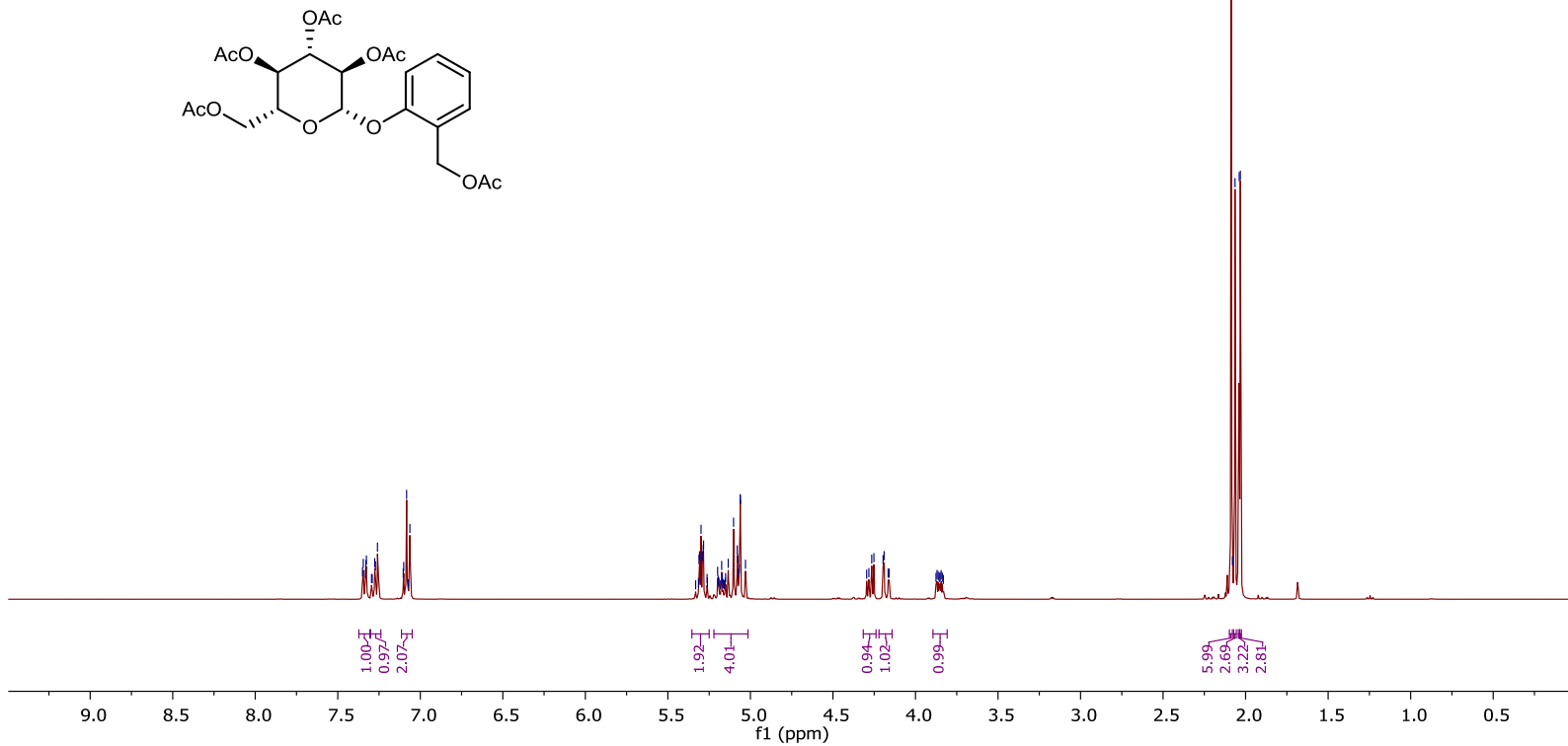

S9 $-{ }^{13} \mathrm{C} \mathrm{NMR}\left(100 \mathrm{MHz}, \mathrm{CDCl}_{3}\right)$

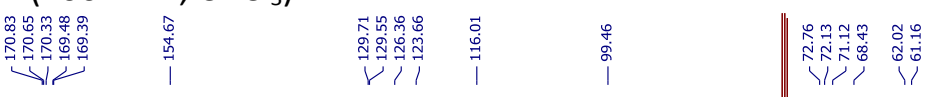

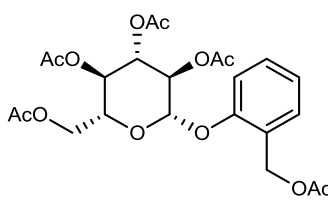

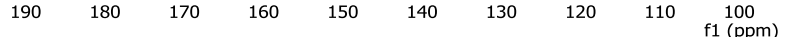

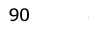

$80 \quad 70$

$60 \quad 50$

$30 \quad 20 \quad 10$ 
<smiles>CC(=O)NC(C)COc1c(C)cccc1N</smiles>

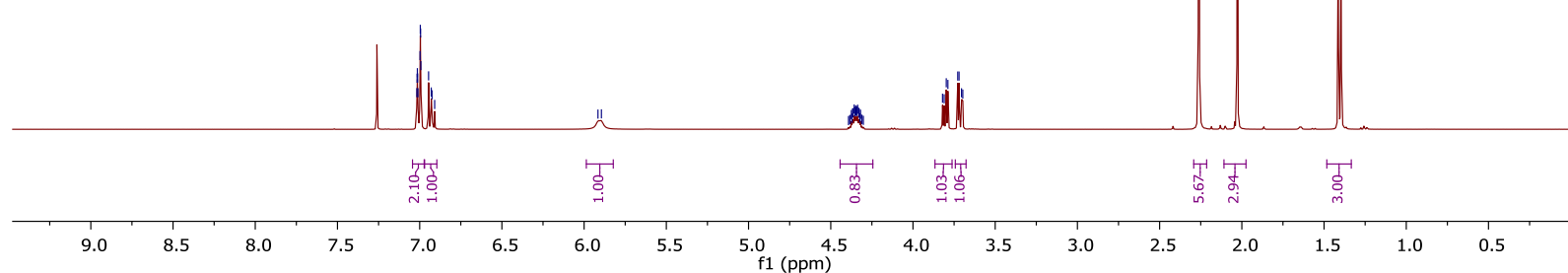

S10 $-{ }^{13} \mathrm{C}$ NMR $\left(100 \mathrm{MHz}, \mathrm{CDCl}_{3}\right)$<smiles>CC(C)NC(C)COc1c(N)cccc1I</smiles>

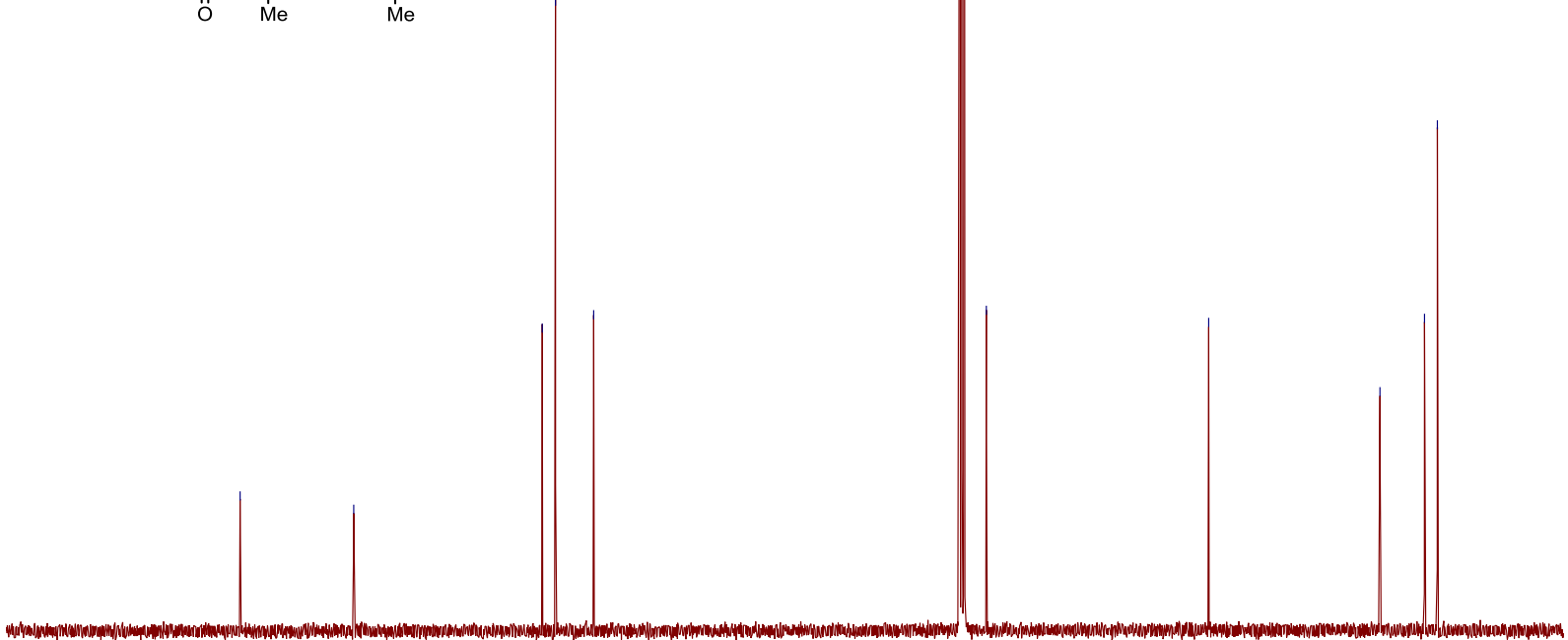




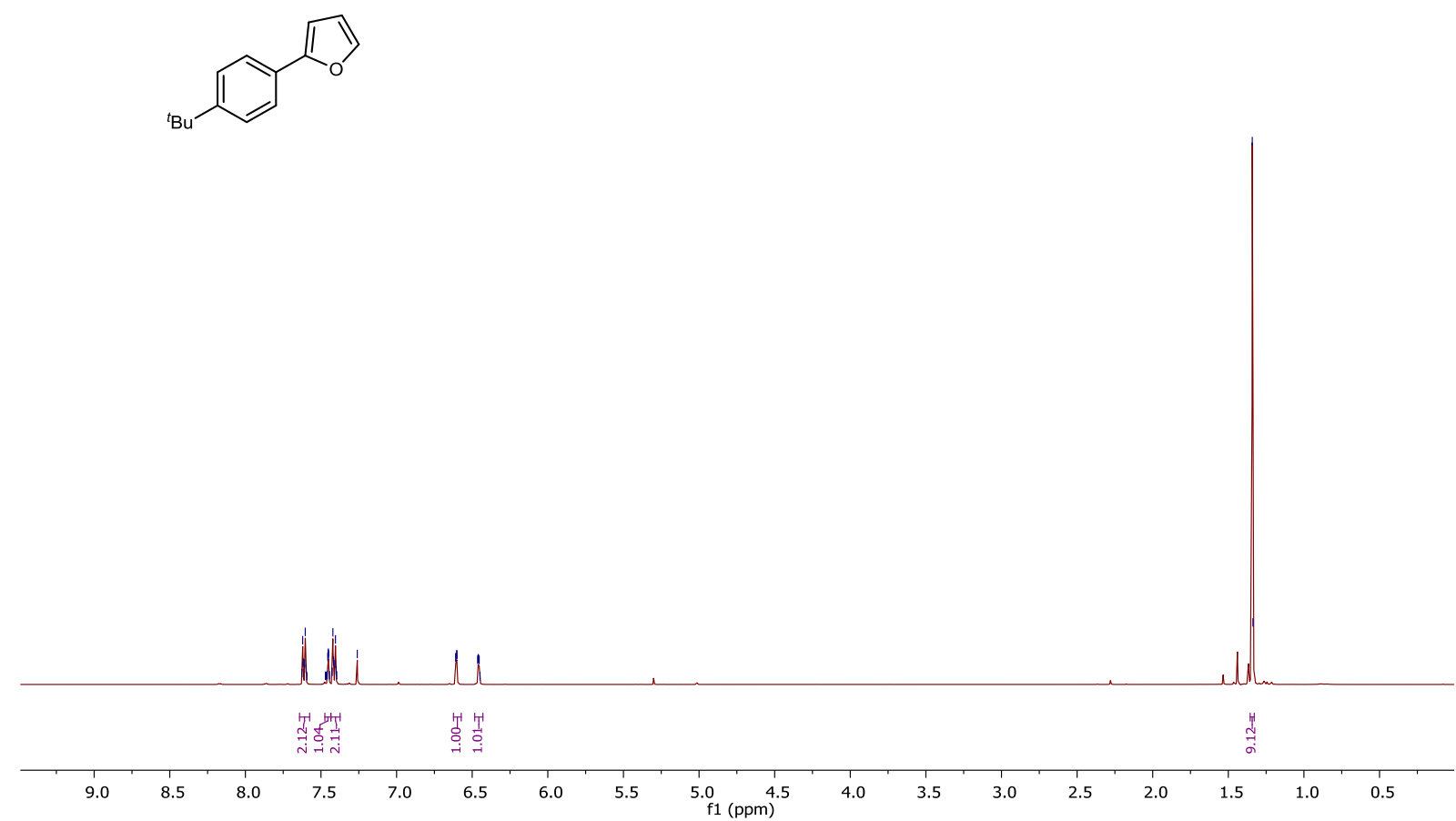

S1 $-{ }^{13} \mathrm{C}$ NMR (125 MHz, $\left.\mathrm{CDCl}_{3}\right)$

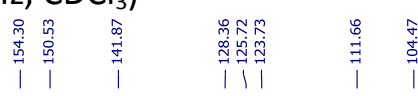<smiles>Brc1ccc(-c2ccco2)cc1</smiles>

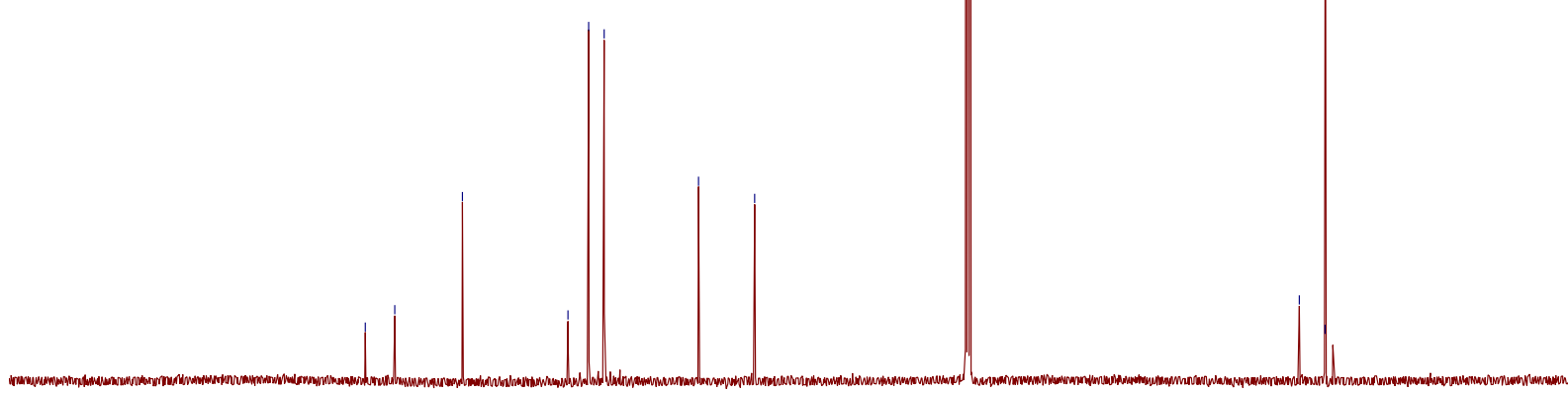


$7-{ }^{1} \mathrm{H}$ NMR (400 MHz, $\mathrm{CDCl}_{3}$ )

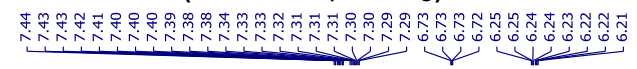

$\stackrel{\infty}{i}$
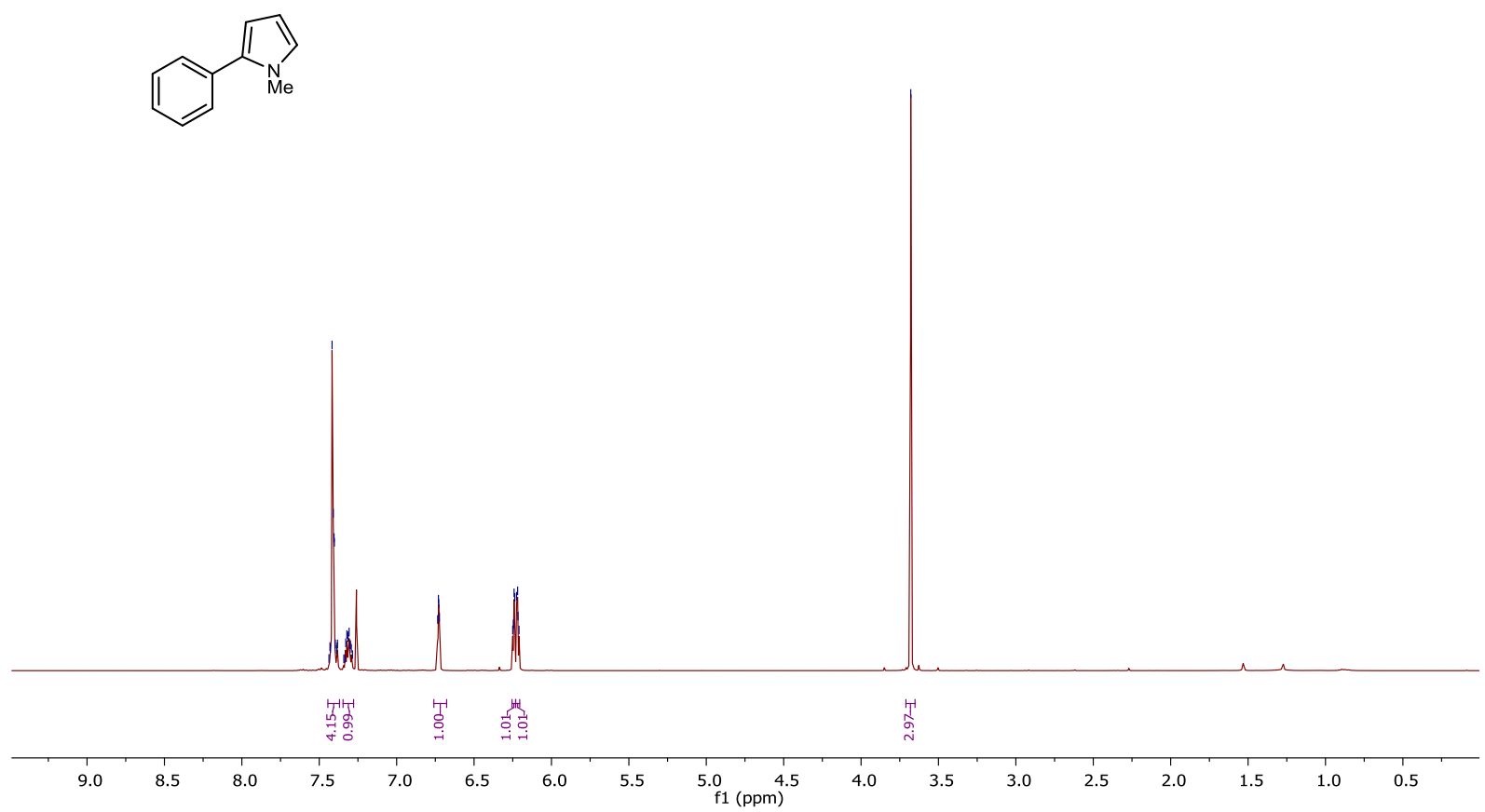

$7-{ }^{13} \mathrm{C} \mathrm{NMR}\left(100 \mathrm{MHz}, \mathrm{CDCl}_{3}\right)$
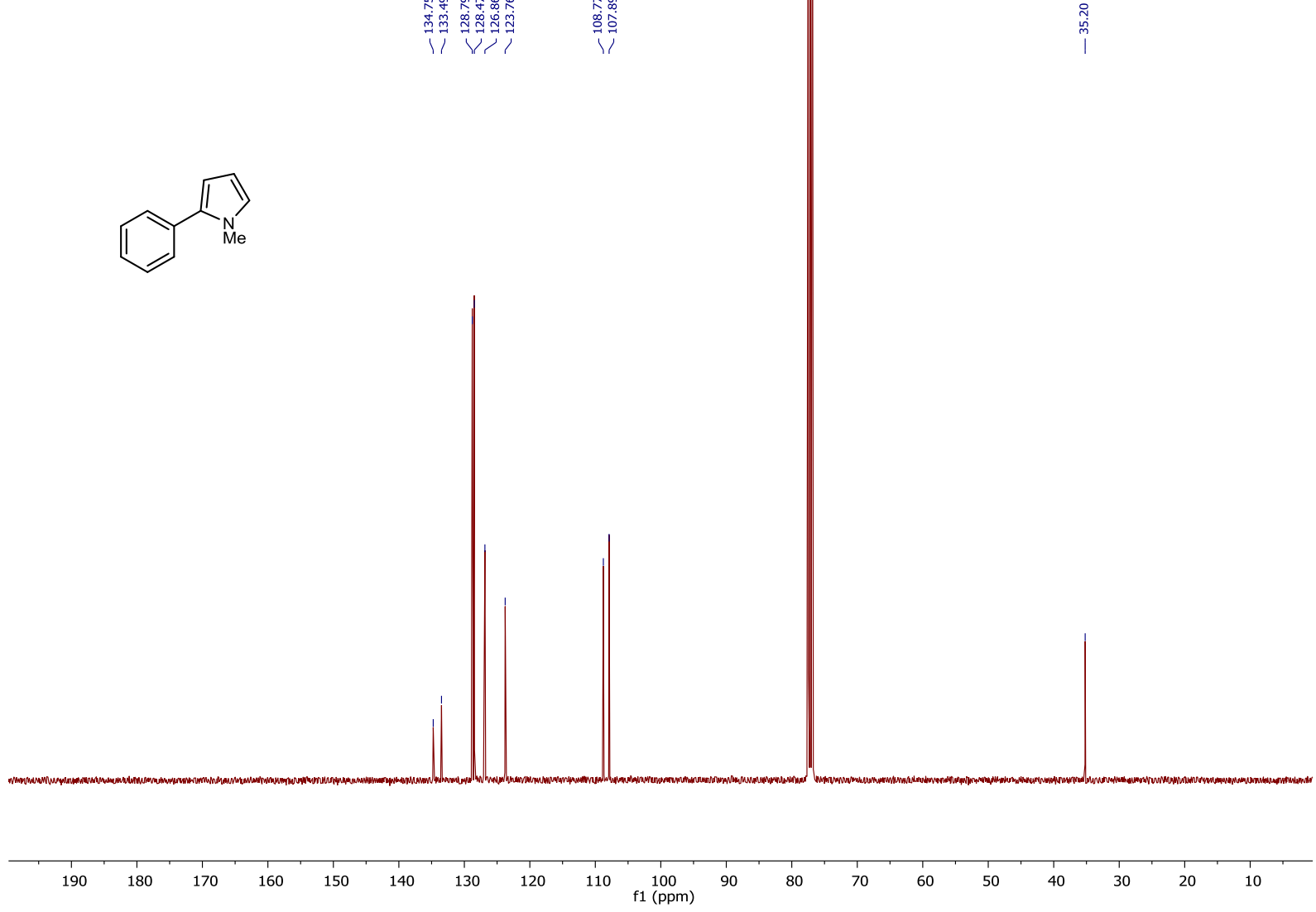

69 
$8-{ }^{1} \mathrm{H}$ NMR $\left(400 \mathrm{MHz}, \mathrm{CDCl}_{3}\right)$

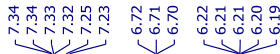<smiles>CCc1ccc(-c2cccn2C)cc1</smiles>

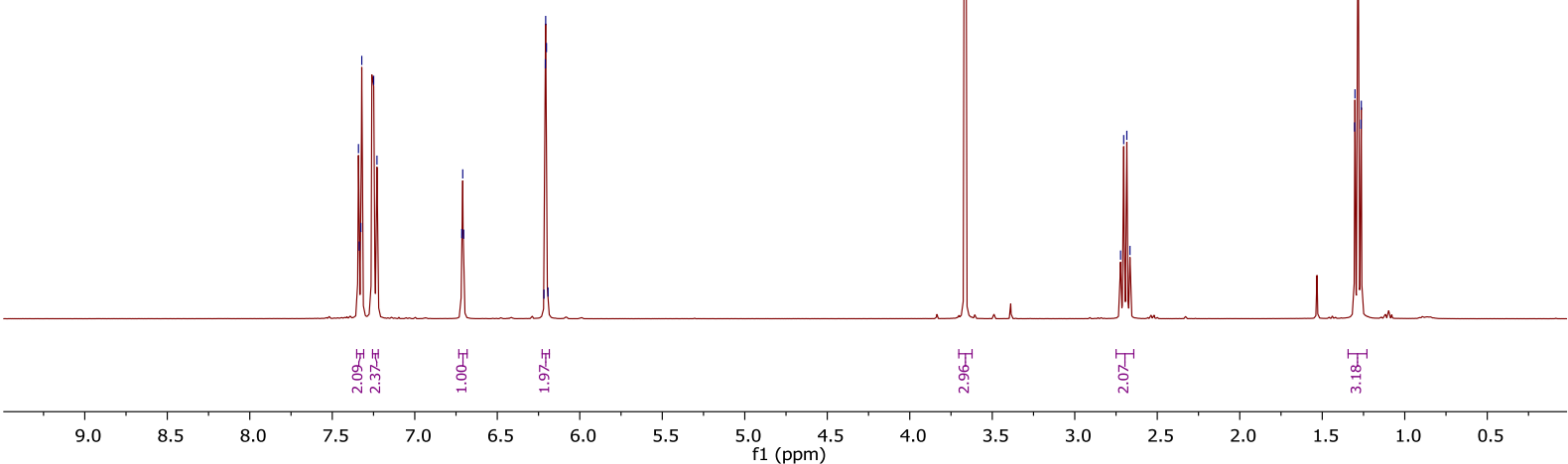

$8-{ }^{13} \mathrm{C}$ NMR (100 MHz, $\left.\mathrm{CDCl}_{3}\right)$
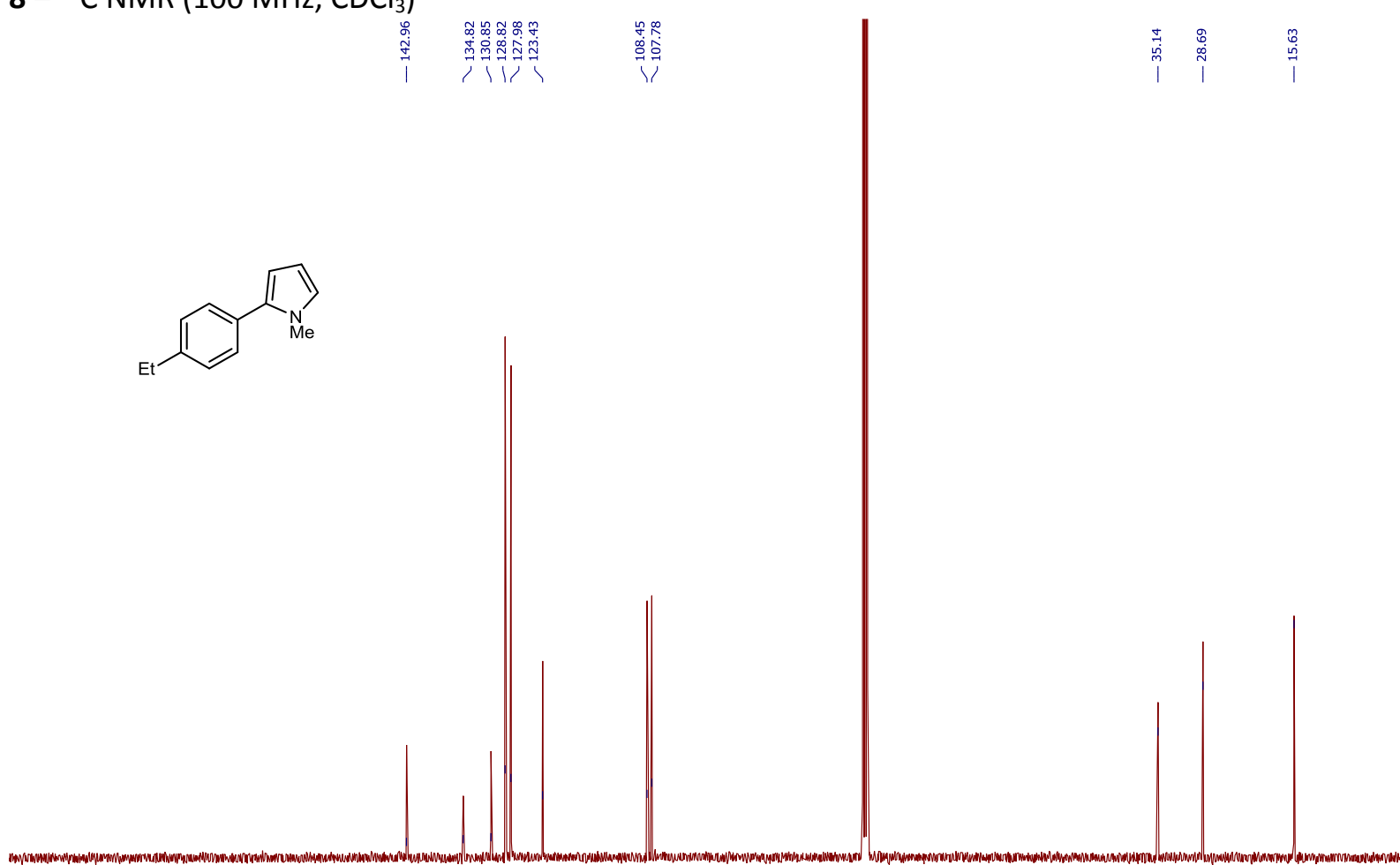

$\begin{array}{llllllllll}190 & 180 & 170 & 160 & 150 & 140 & 130 & 120 & 110 & \begin{array}{c}100 \\ \mathrm{f} 1(\mathrm{ppm})\end{array}\end{array}$

80

10 
$9-{ }^{1} \mathrm{H}$ NMR $\left(400 \mathrm{MHz}, \mathrm{CDCl}_{3}\right)$

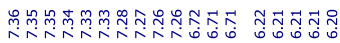

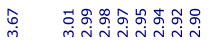

국<smiles></smiles>

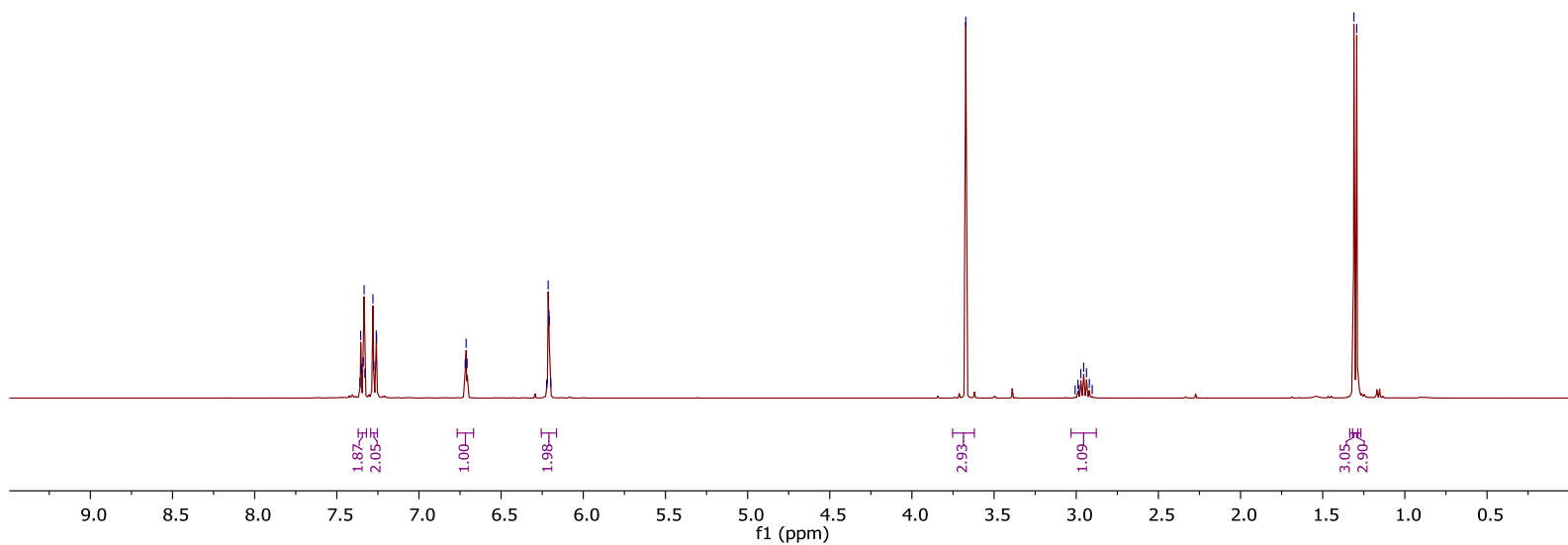

$9-{ }^{13} \mathrm{CNMR}\left(100 \mathrm{MHz}, \mathrm{CDCl}_{3}\right)$
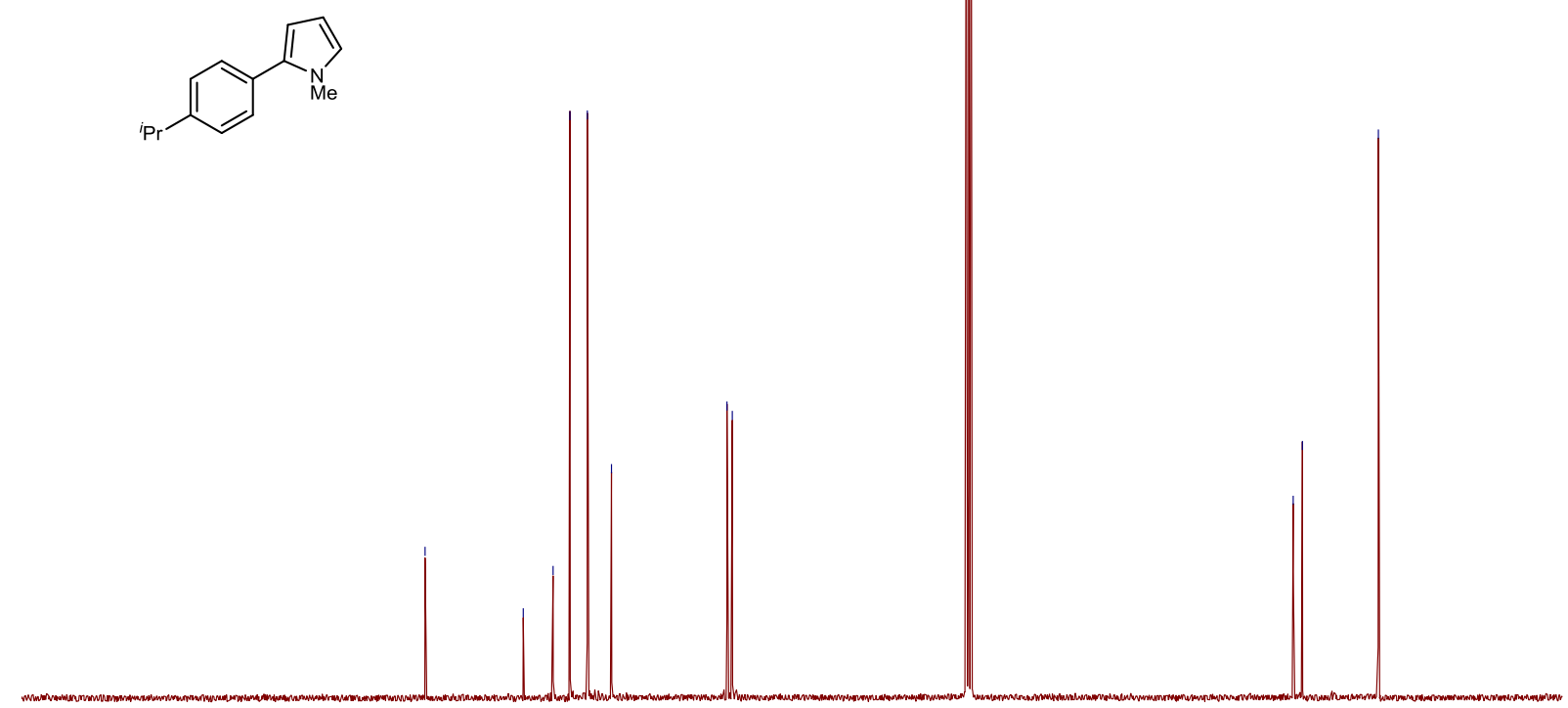

190

$180 \quad 170$

$160 \quad 150$

140

$10 \begin{gathered}100 \\ \mathrm{f} 1(\mathrm{ppm})\end{gathered}$

90

70

50

30

10 
$10-{ }^{1} \mathrm{H}$ NMR $\left(400 \mathrm{MHz}, \mathrm{CDCl}_{3}\right)$

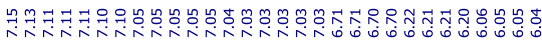<smiles>Cc1ccc(C2=NCC2)c(C)c1</smiles>

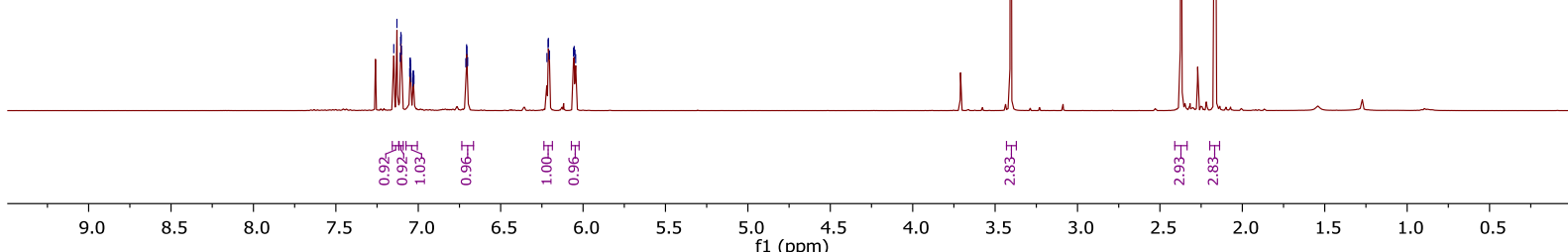

$10-{ }^{13} \mathrm{CNMR}\left(100 \mathrm{MHz}, \mathrm{CDCl}_{3}\right)$ 年<smiles>Cc1ccc(C2CCN2)c(C)c1</smiles>

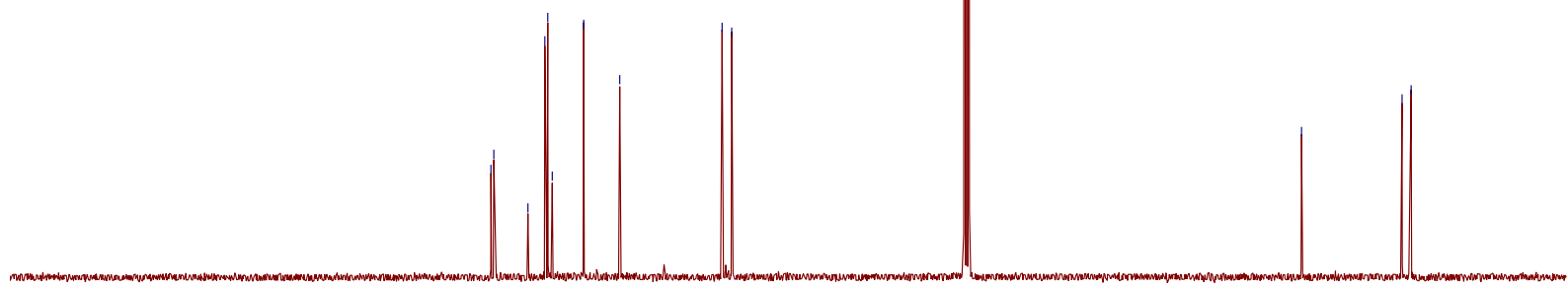




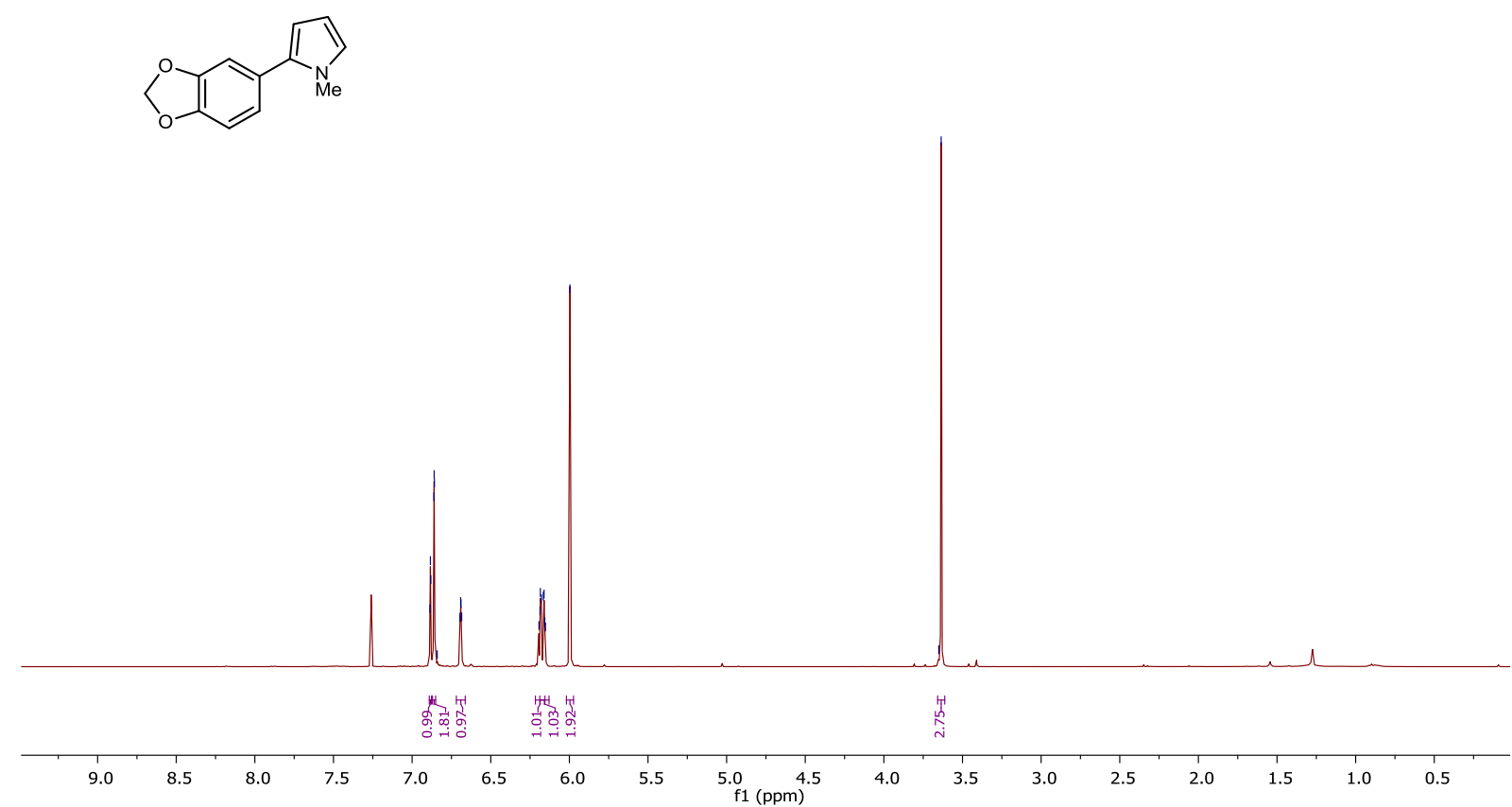

$11-{ }^{13} \mathrm{C}$ NMR $\left(100 \mathrm{MHz}, \mathrm{CDCl}_{3}\right)$<smiles></smiles>

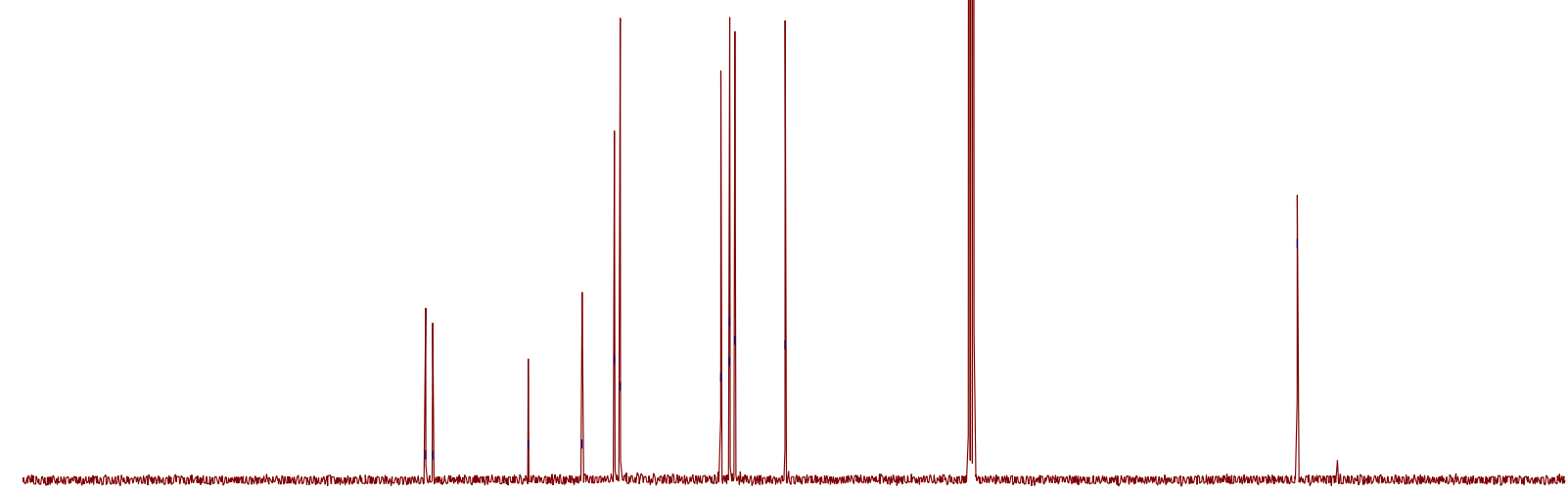


$12-{ }^{1} \mathrm{H}$ NMR (400 MHz, $\mathrm{CDCl}_{3}$ )

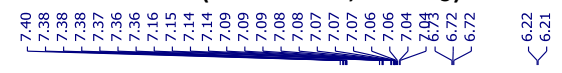

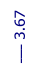
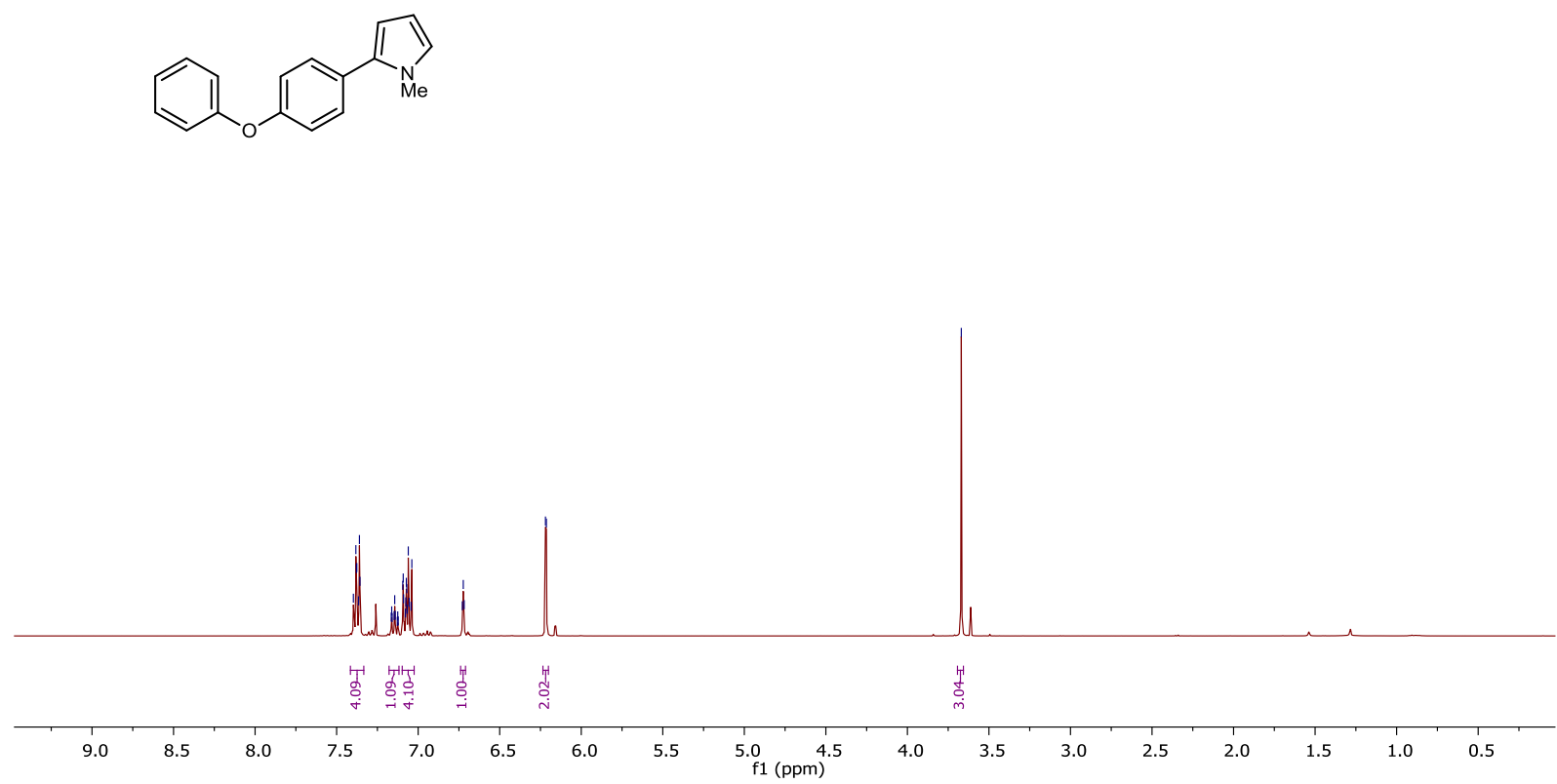

$12-{ }^{13} \mathrm{C}$ NMR $\left(100 \mathrm{MHz}, \mathrm{CDCl}_{3}\right)$

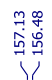

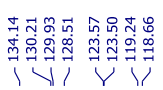

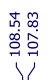

范
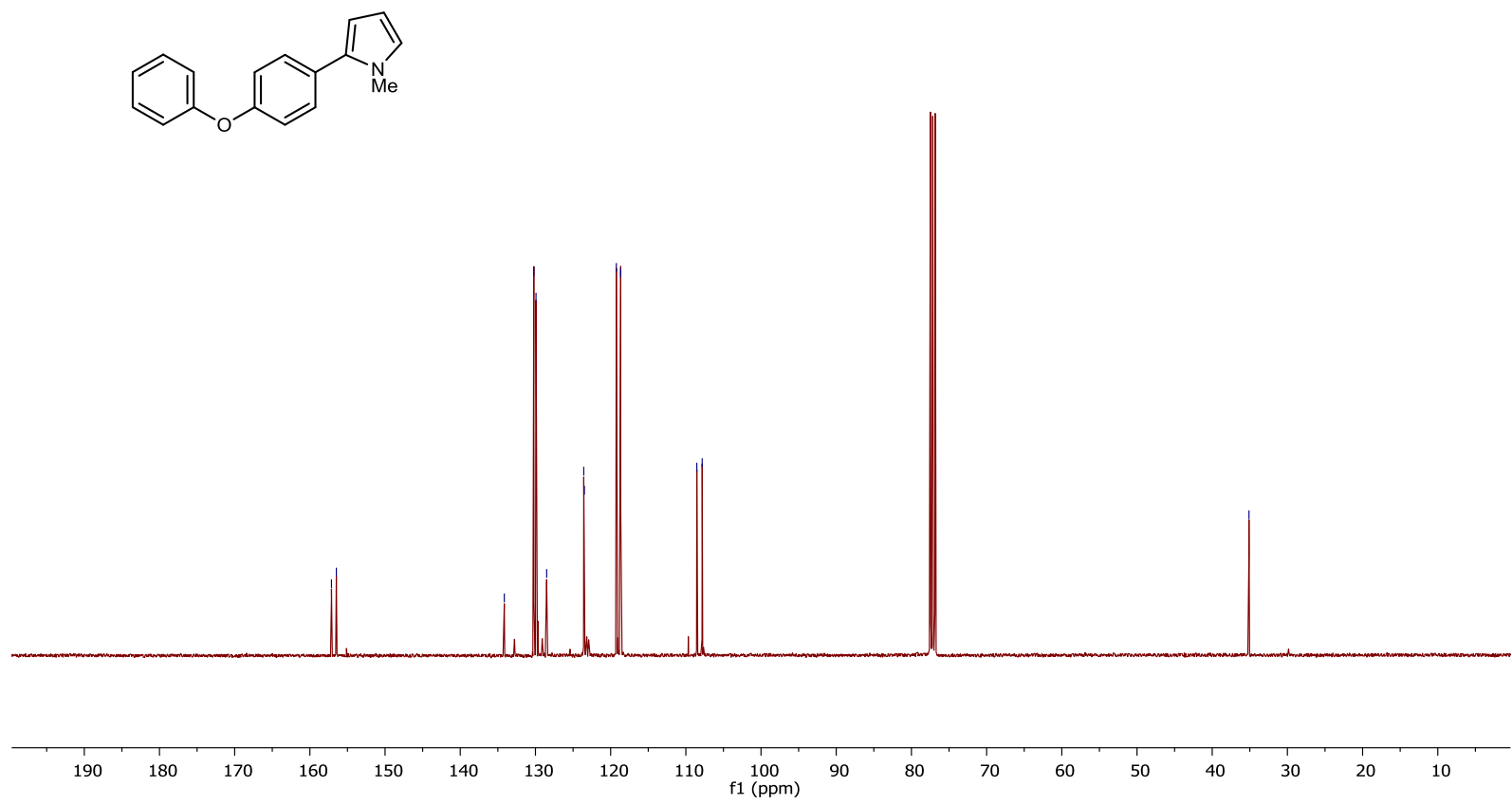

75 
$13-{ }^{1} \mathrm{H}$ NMR $\left(400 \mathrm{MHz}, \mathrm{CDCl}_{3}\right)$

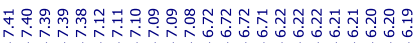<smiles></smiles>

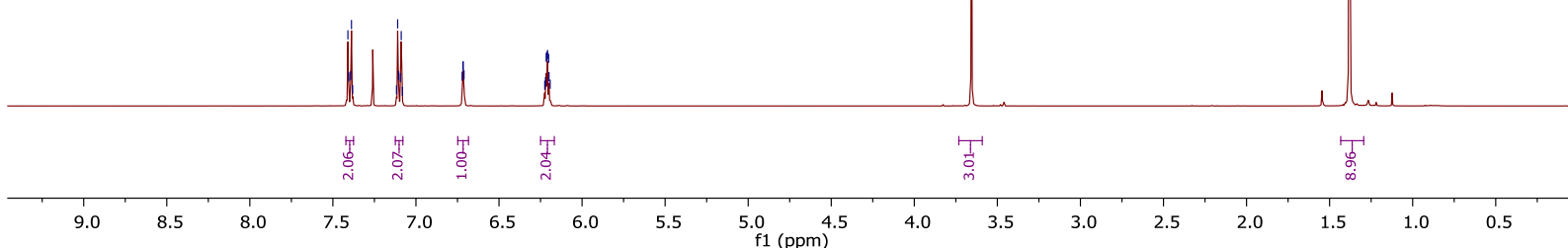

$13-{ }^{13} \mathrm{C}$ NMR $\left(100 \mathrm{MHz}, \mathrm{CDCl}_{3}\right)$

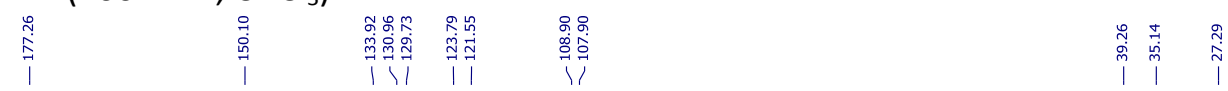<smiles></smiles>

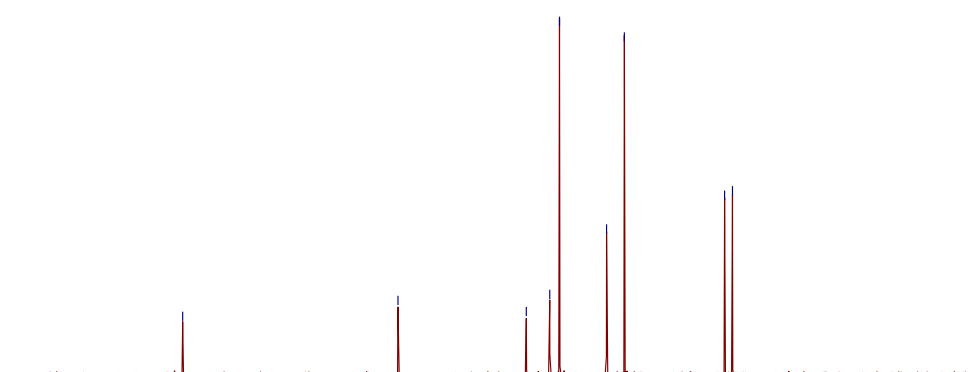

$$
\mid
$$

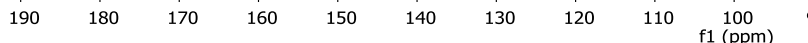

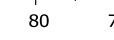

6050

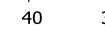

$20 \quad 10$ 
$14-{ }^{1} \mathrm{H} N M R\left(400 \mathrm{MHz}, \mathrm{CDCl}_{3}\right)$

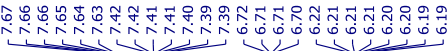<smiles>O=C1CCCC1c1ccc(C2=CCCC2)cc1</smiles>

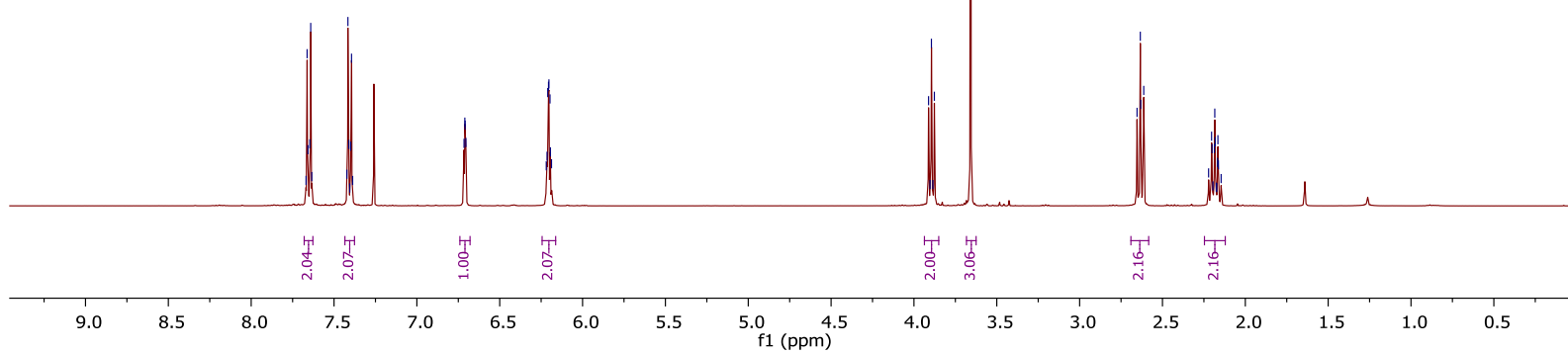

$14-{ }^{13} \mathrm{C}$ NMR (100 MHz, $\left.\mathrm{CDCl}_{3}\right)$<smiles>O=C1CCCN1c1ccc(-c2cccn2-c2ccccc2)cc1</smiles>

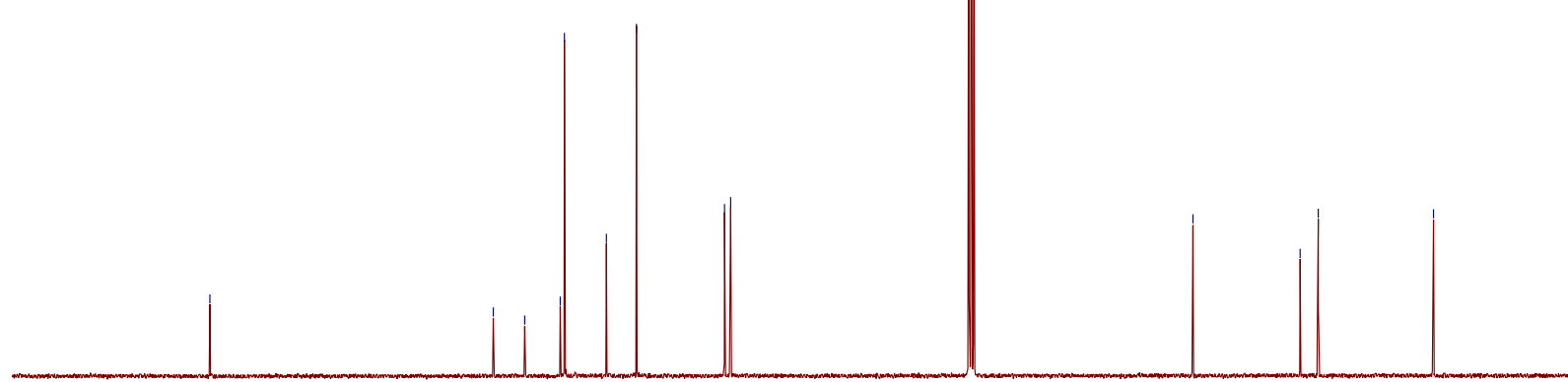

$\begin{array}{llc}120 & 110 & 100 \\ \mathrm{f} 1(\mathrm{ppm})\end{array}$

80

70

$60 \quad 50$

$40 \quad 30 \quad 20 \quad 10$ 
$15-{ }^{1} \mathrm{H}$ NMR $\left(400 \mathrm{MHz}, \mathrm{CDCl}_{3}\right)$

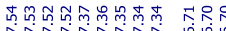<smiles></smiles>

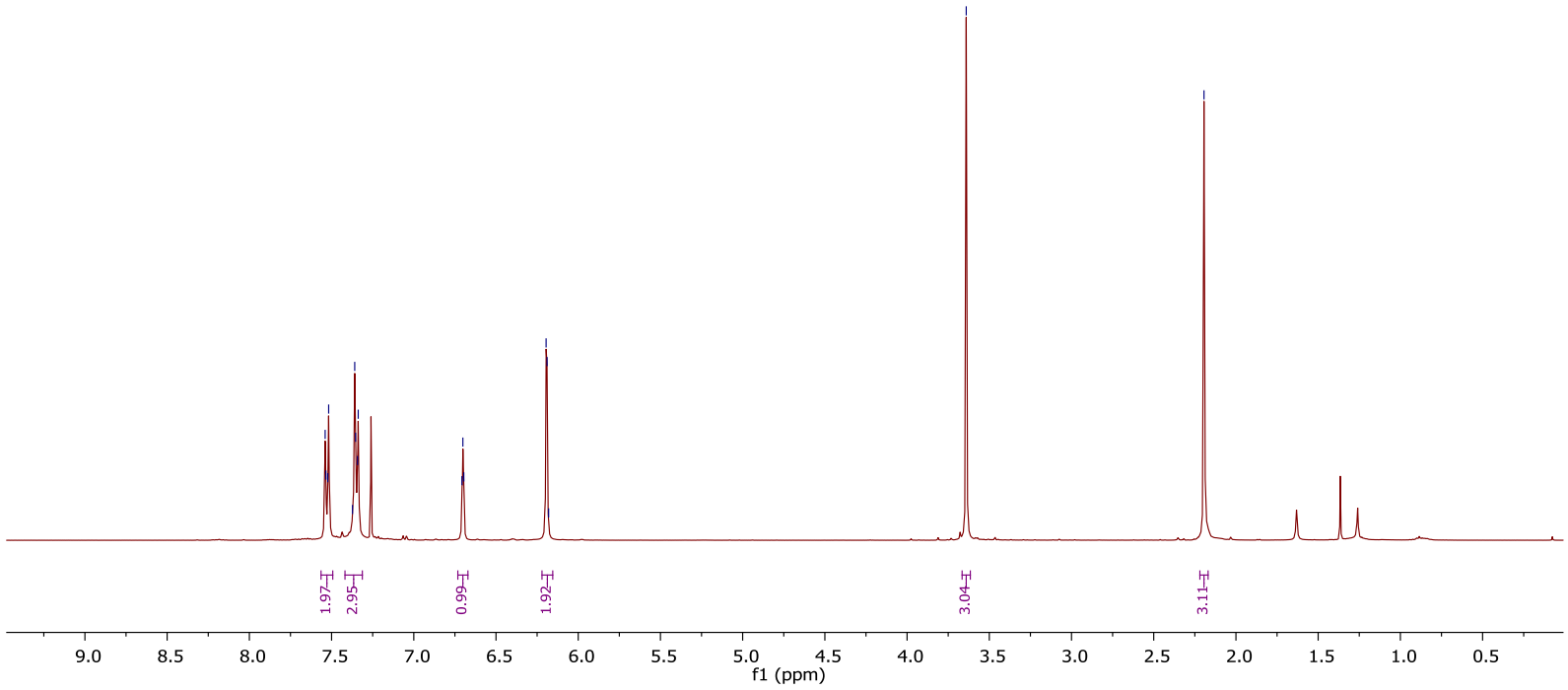

$15-{ }^{13} \mathrm{C}$ NMR $\left(100 \mathrm{MHz}, \mathrm{CDCl}_{3}\right)$<smiles></smiles>

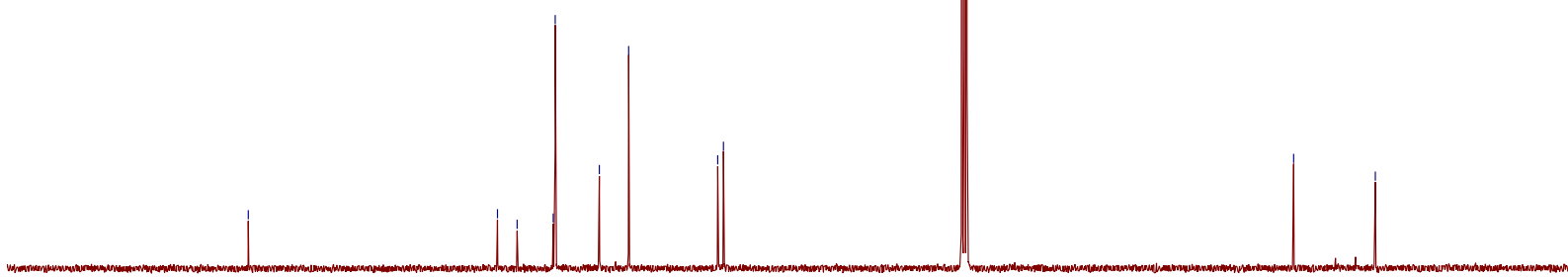

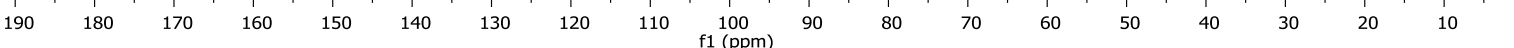



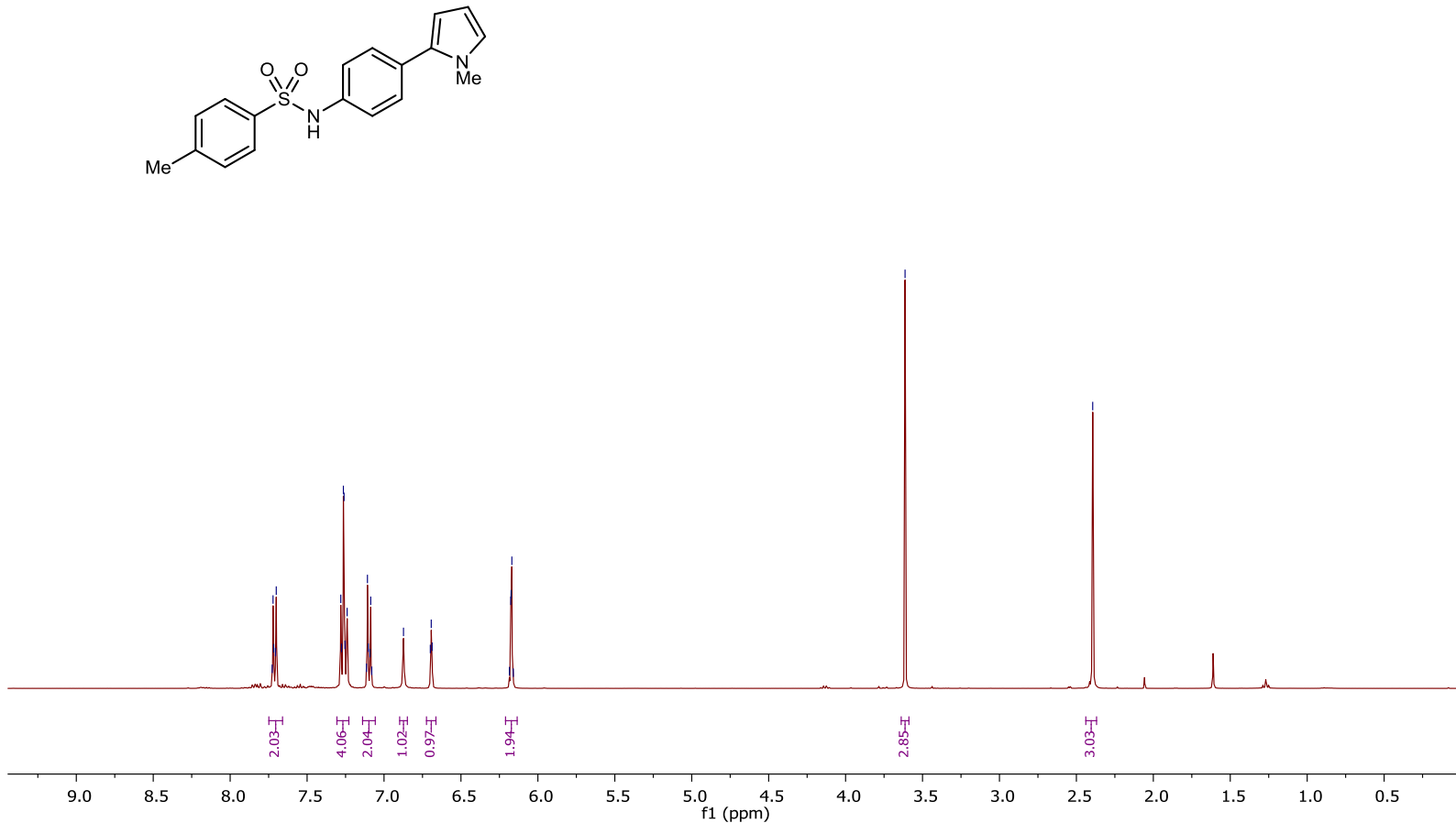

$16-{ }^{13} \mathrm{C} \mathrm{NMR}\left(100 \mathrm{MHz}, \mathrm{CDCl}_{3}\right)$

|<smiles>Cn1cccc1-c1ccc(NS(=O)(=O)c2ccc([N+](=O)[O-])cc2)cc1</smiles>

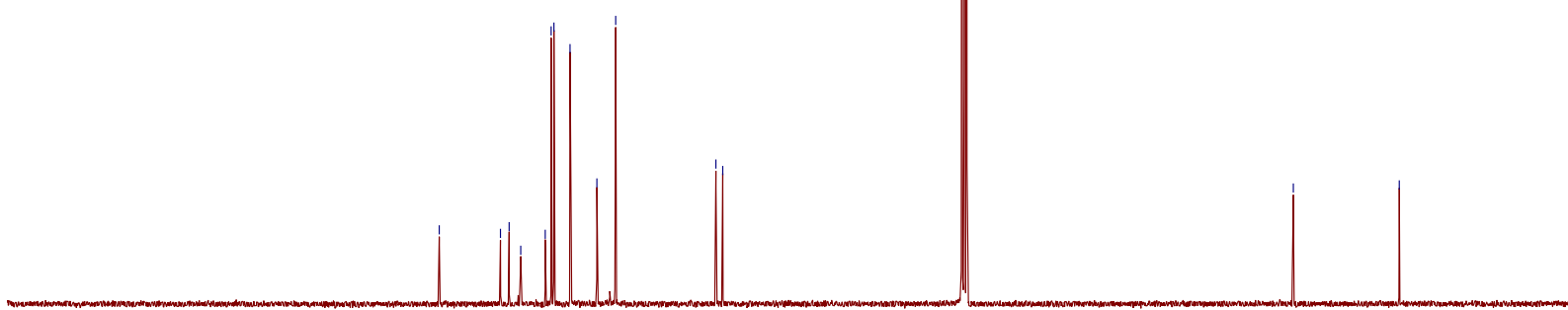


$17-{ }^{1} \mathrm{H}$ NMR $\left(400 \mathrm{MHz}, \mathrm{CDCl}_{3}\right)$

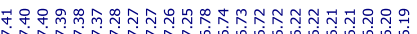
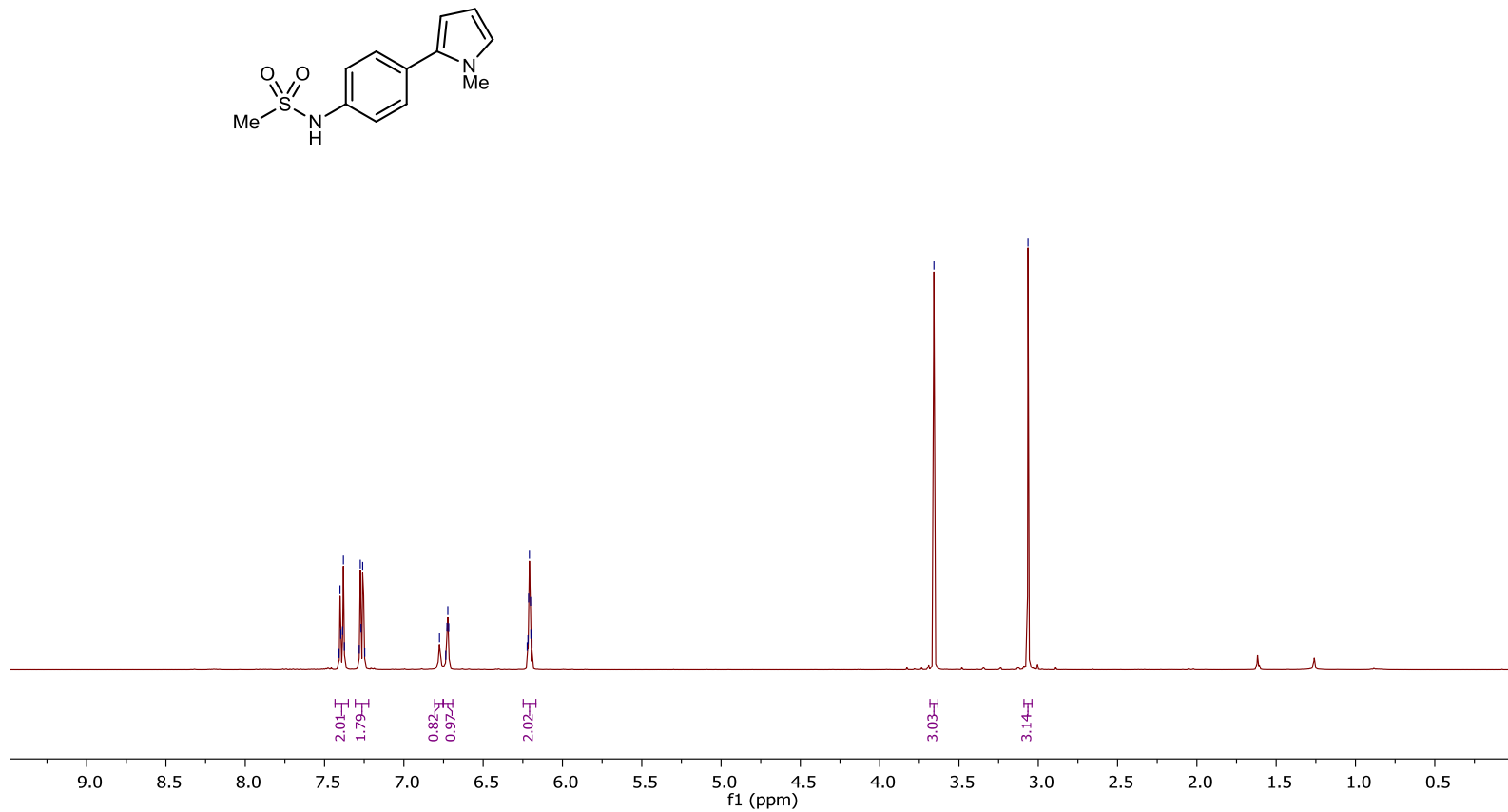

$17-{ }^{13} \mathrm{CNMR}\left(100 \mathrm{MHz}, \mathrm{CDCl}_{3}\right)$

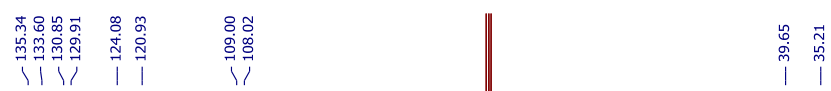<smiles></smiles>

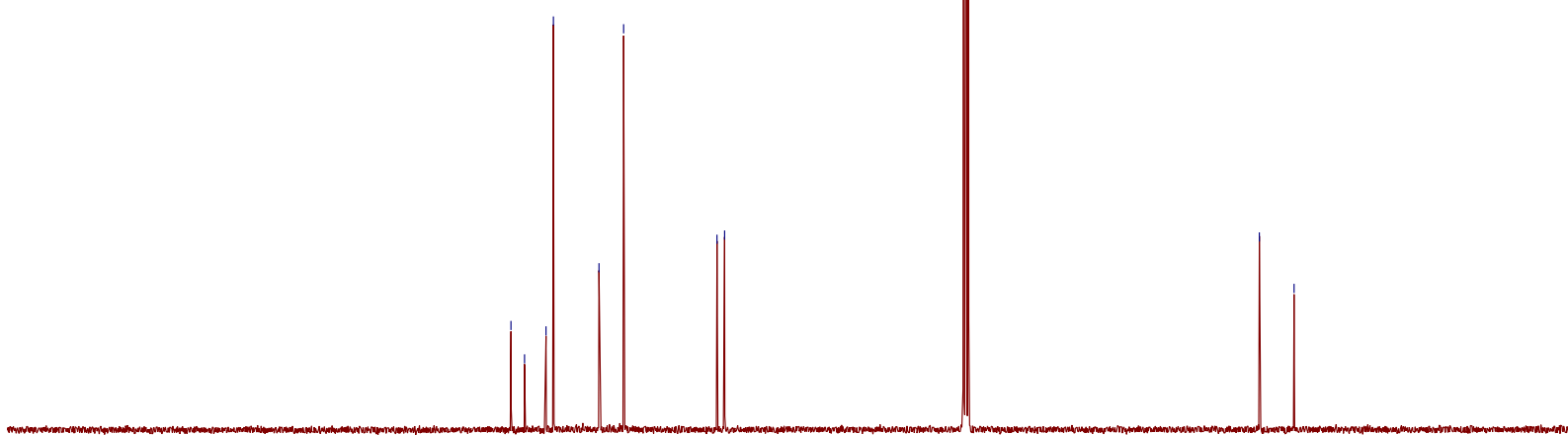

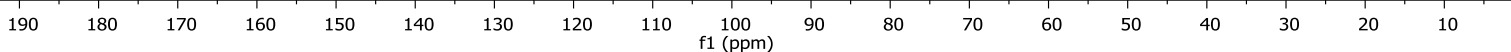


$18-{ }^{1} \mathrm{H}$ NMR $\left(400 \mathrm{MHz}, \mathrm{CDCl}_{3}\right)$

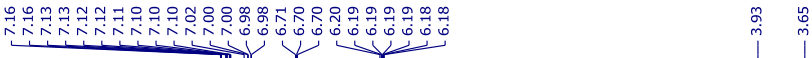

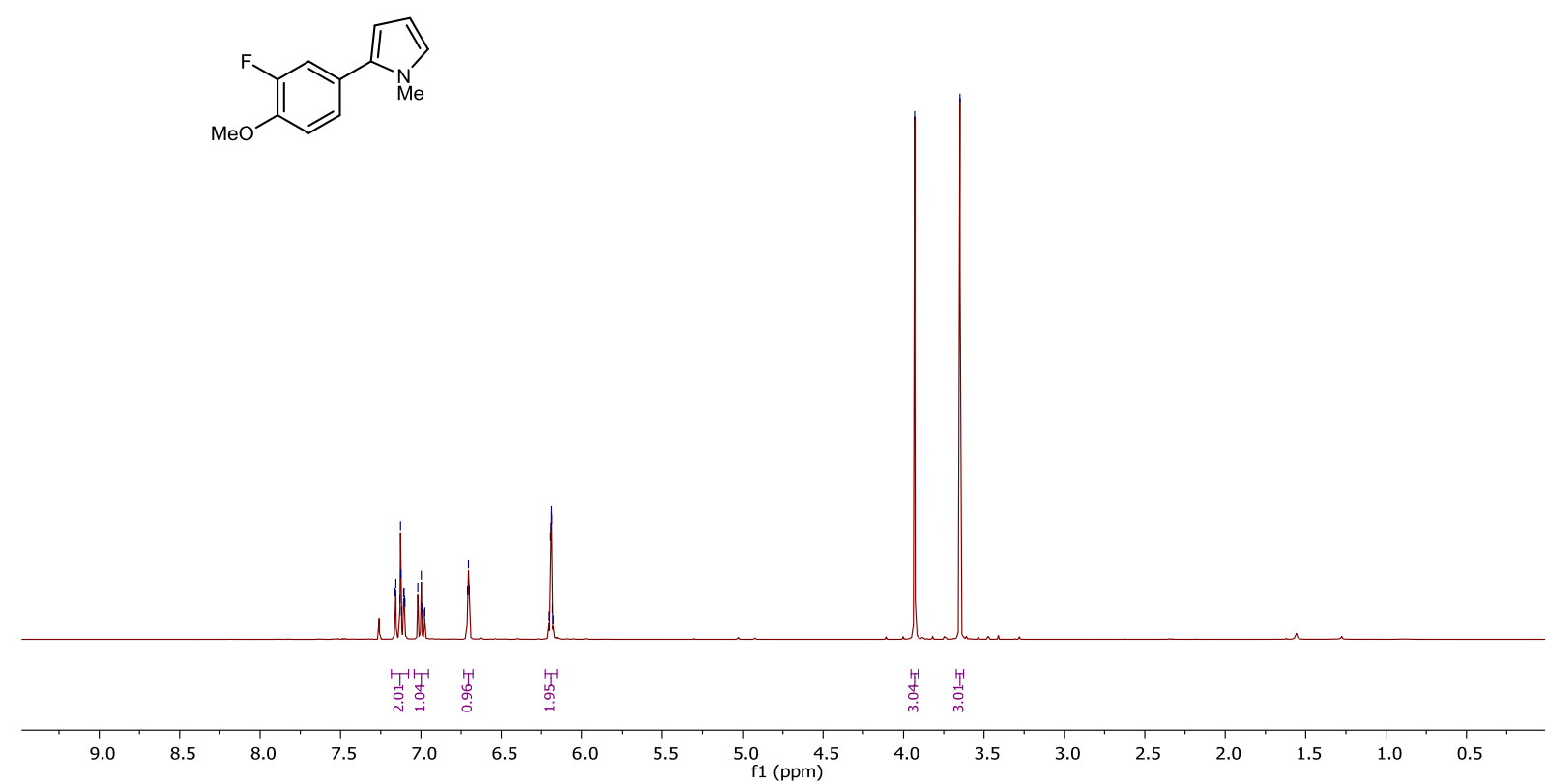

$18-{ }^{13} \mathrm{C}$ NMR $\left(100 \mathrm{MHz}, \mathrm{CDCl}_{3}\right)$

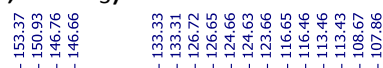

$1 Y$ Y Y Y Y V<smiles></smiles>

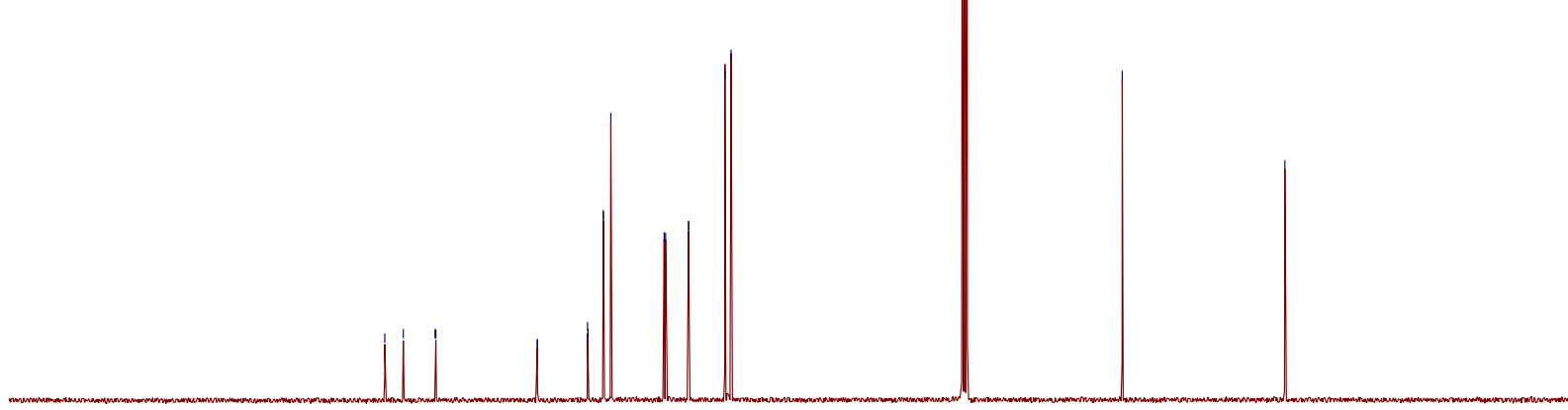

$\begin{array}{lllllllllllllllllllll}200 & 190 & 180 & 170 & 160 & 150 & 140 & 130 & 120 & 110 & 100 & 90 & 80 & 70 & 60 & 50 & 40 & 30 & 20 & 10 & 0\end{array}$ 
$18-{ }^{19} \mathrm{~F} \mathrm{NMR}\left(376 \mathrm{MHz}, \mathrm{CDCl}_{3}\right)$

ì<smiles>Cn1cccc1-c1ccc(O)c(F)c1</smiles>

$\begin{array}{lllllllllllllllllllllllllllll}10 & 0 & -10 & -20 & -30 & -40 & -50 & -60 & -70 & -80 & -90 & -100 & -110 & -120 & -130 & -140 & -150 & -160 & -170 & -180 & -190 & -200 & -210\end{array}$ 
$19-{ }^{1} \mathrm{H}$ NMR $\left(400 \mathrm{MHz}, \mathrm{CDCl}_{3}\right)$

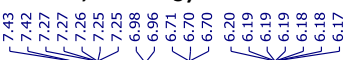
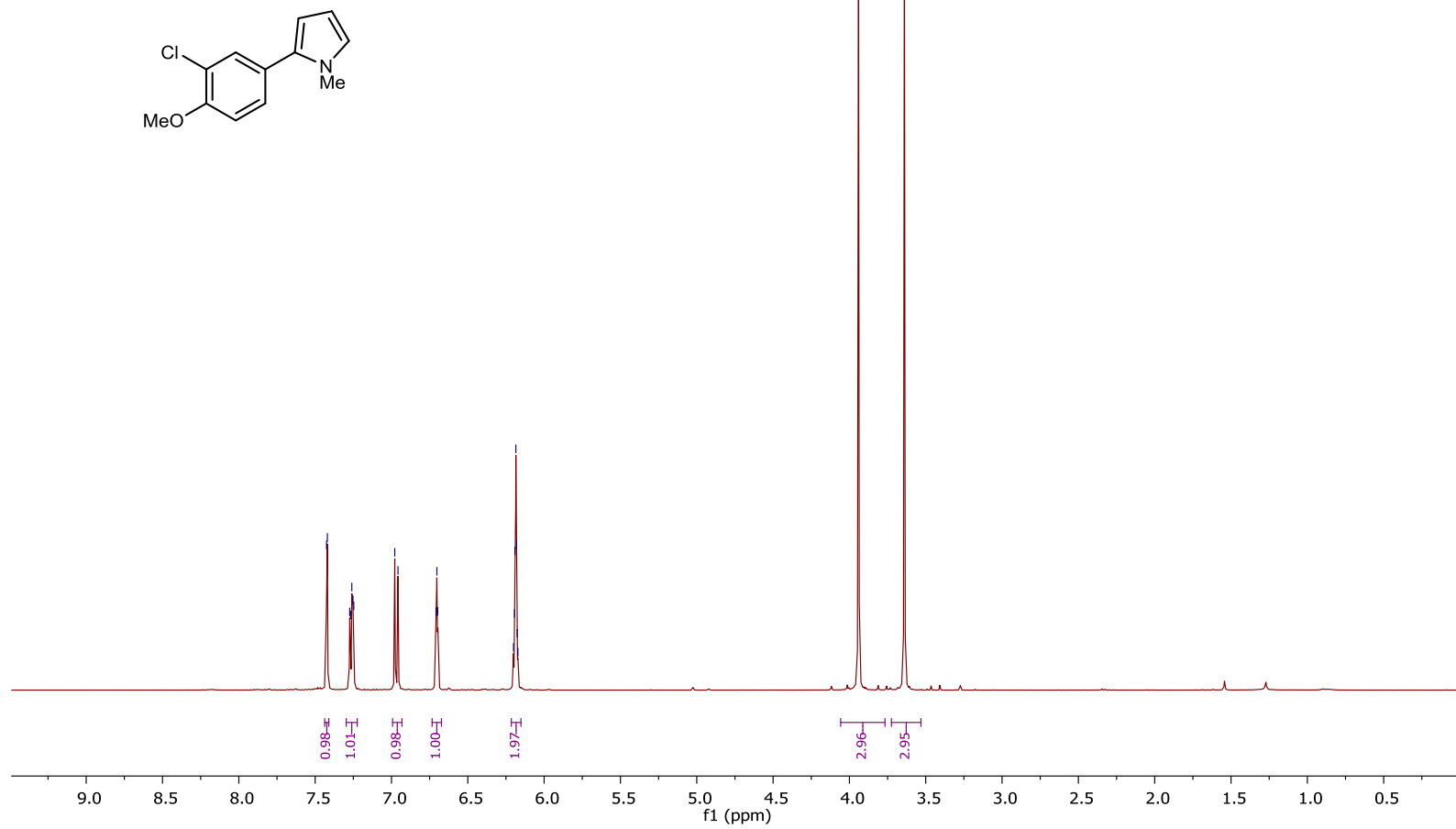

$19-{ }^{13} \mathrm{C}$ NMR $\left(100 \mathrm{MHz}, \mathrm{CDCl}_{3}\right)$

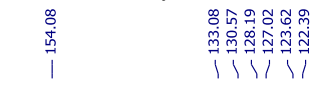

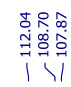

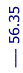

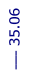
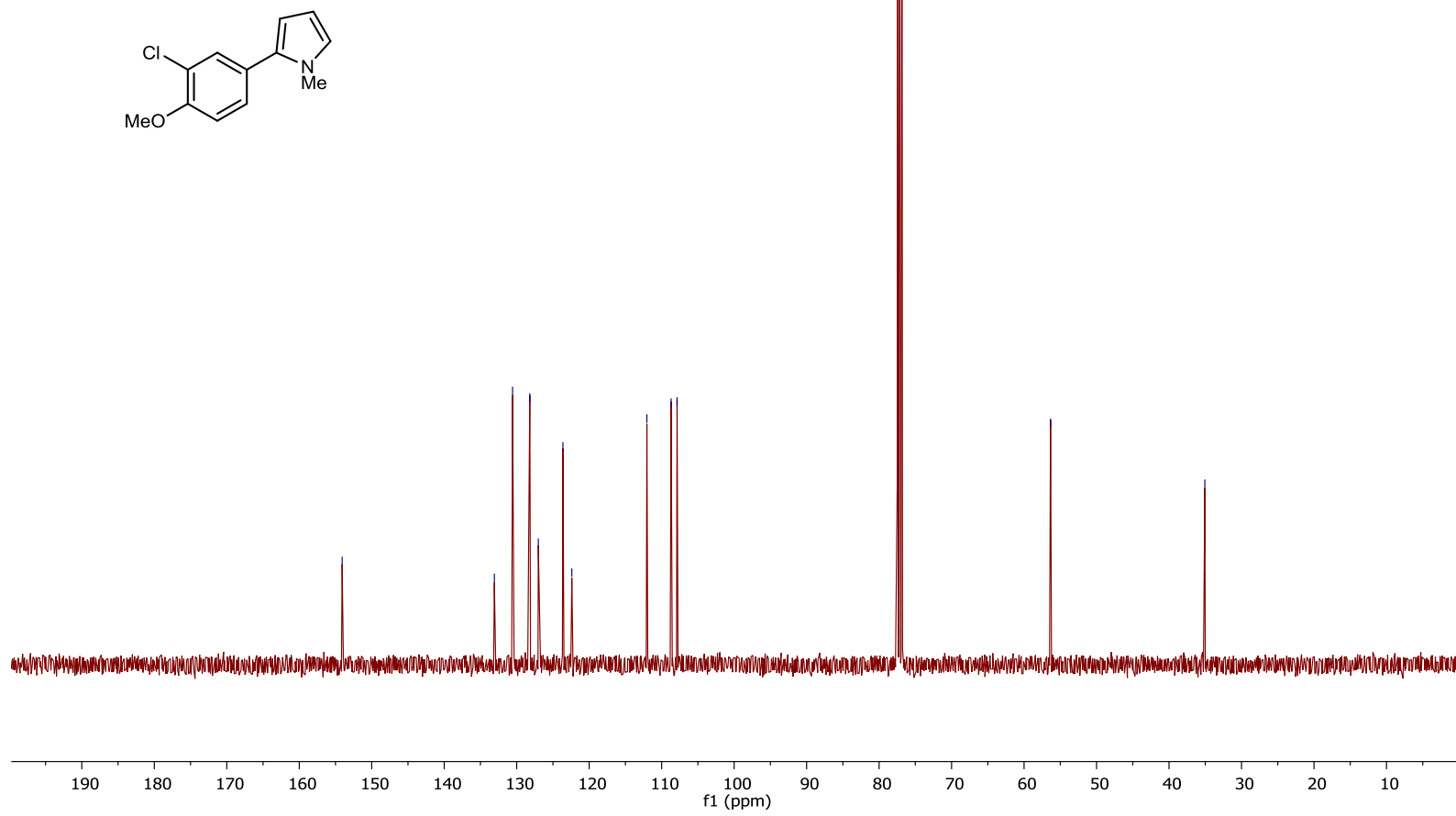

83 
$20-{ }^{1} \mathrm{H}$ NMR $\left(400 \mathrm{MHz}, \mathrm{CDCl}_{3}\right)$

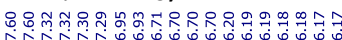

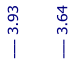

Meo

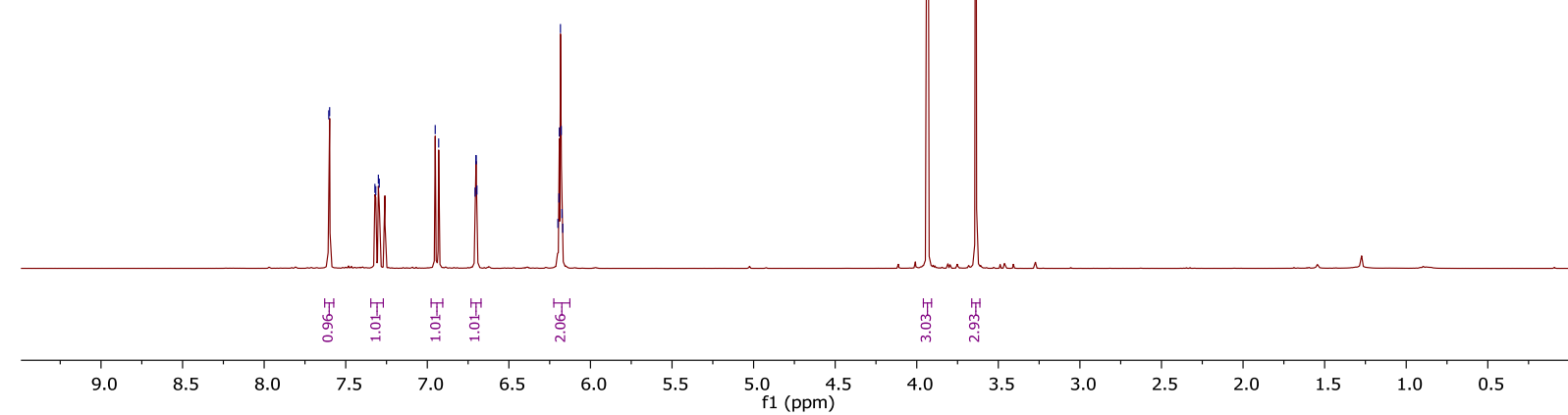

$20-{ }^{13} \mathrm{CNMR}\left(100 \mathrm{MHz}, \mathrm{CDCl}_{3}\right)$

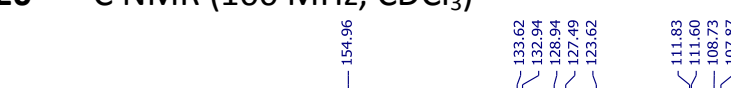<smiles>COc1ccc(-c2cccn2C)cc1Br</smiles>

meo
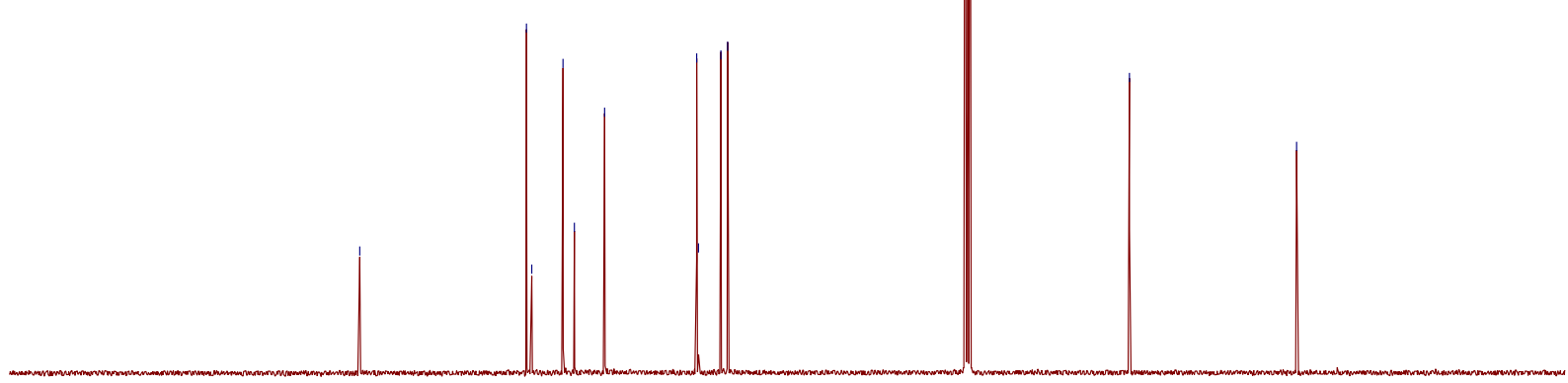

$\begin{array}{llllllllll}190 & 180 & 170 & 160 & 150 & 140 & 130 & 120 & 110 & \begin{array}{l}100 \\ \mathrm{f} 1(\mathrm{ppm})\end{array}\end{array}$ 
$21-{ }^{1} \mathrm{H}$ NMR $\left(400 \mathrm{MHz}, \mathrm{CDCl}_{3}\right)$

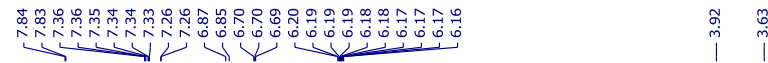

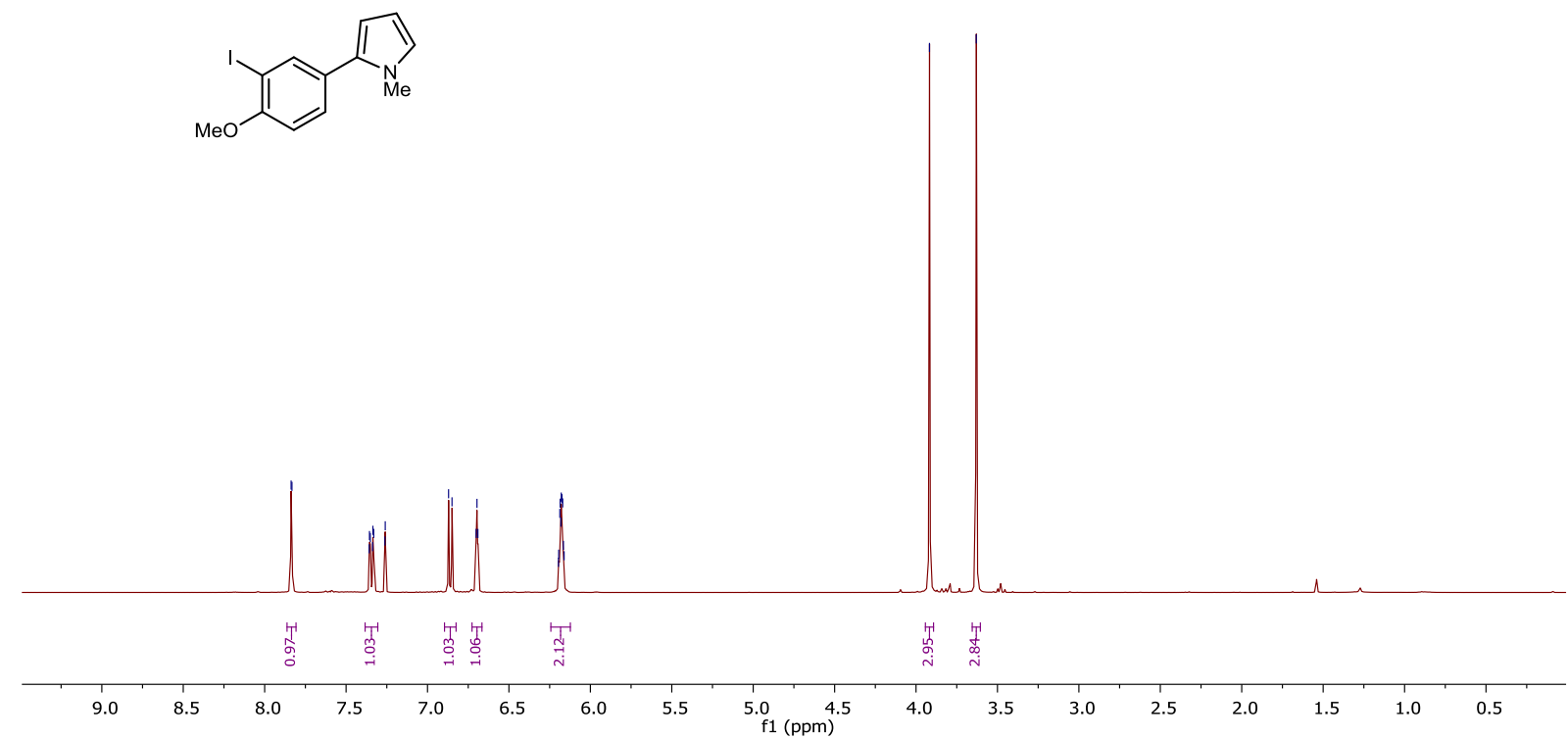

$21-{ }^{13} \mathrm{C}$ NMR $\left(100 \mathrm{MHz}, \mathrm{CDCl}_{3}\right.$ )

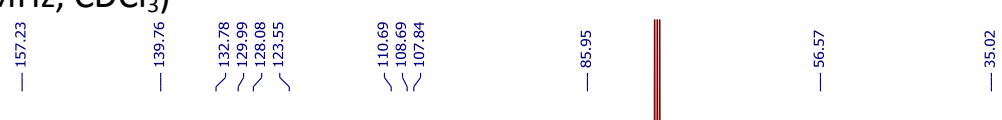<smiles></smiles>

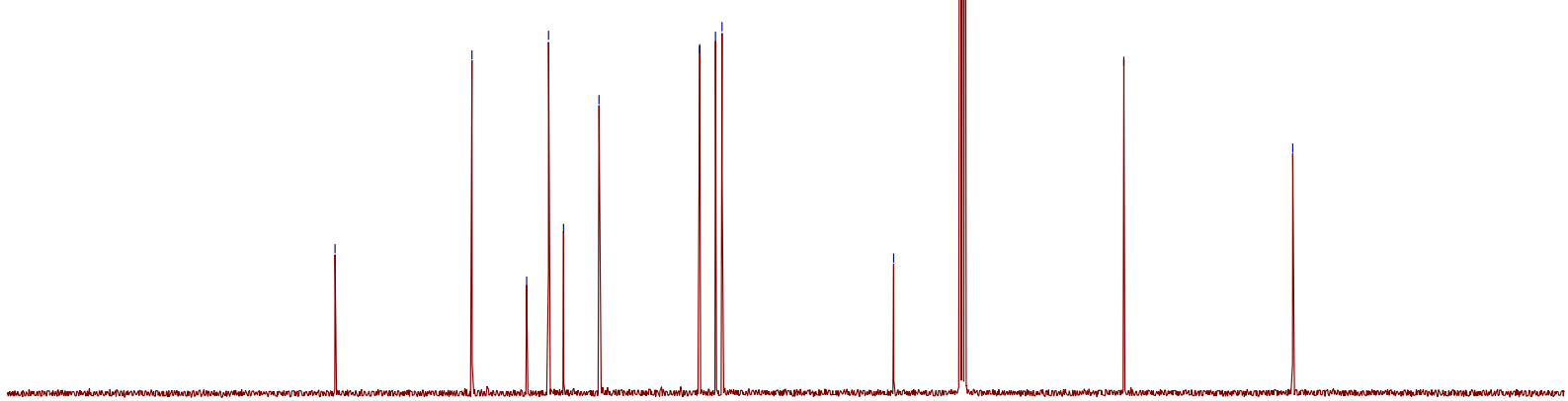

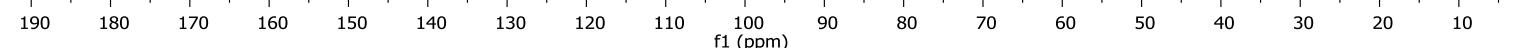


$22-{ }^{1} \mathrm{H}$ NMR $\left(400 \mathrm{MHz}, \mathrm{CDCl}_{3}\right)$

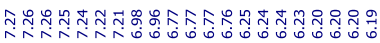
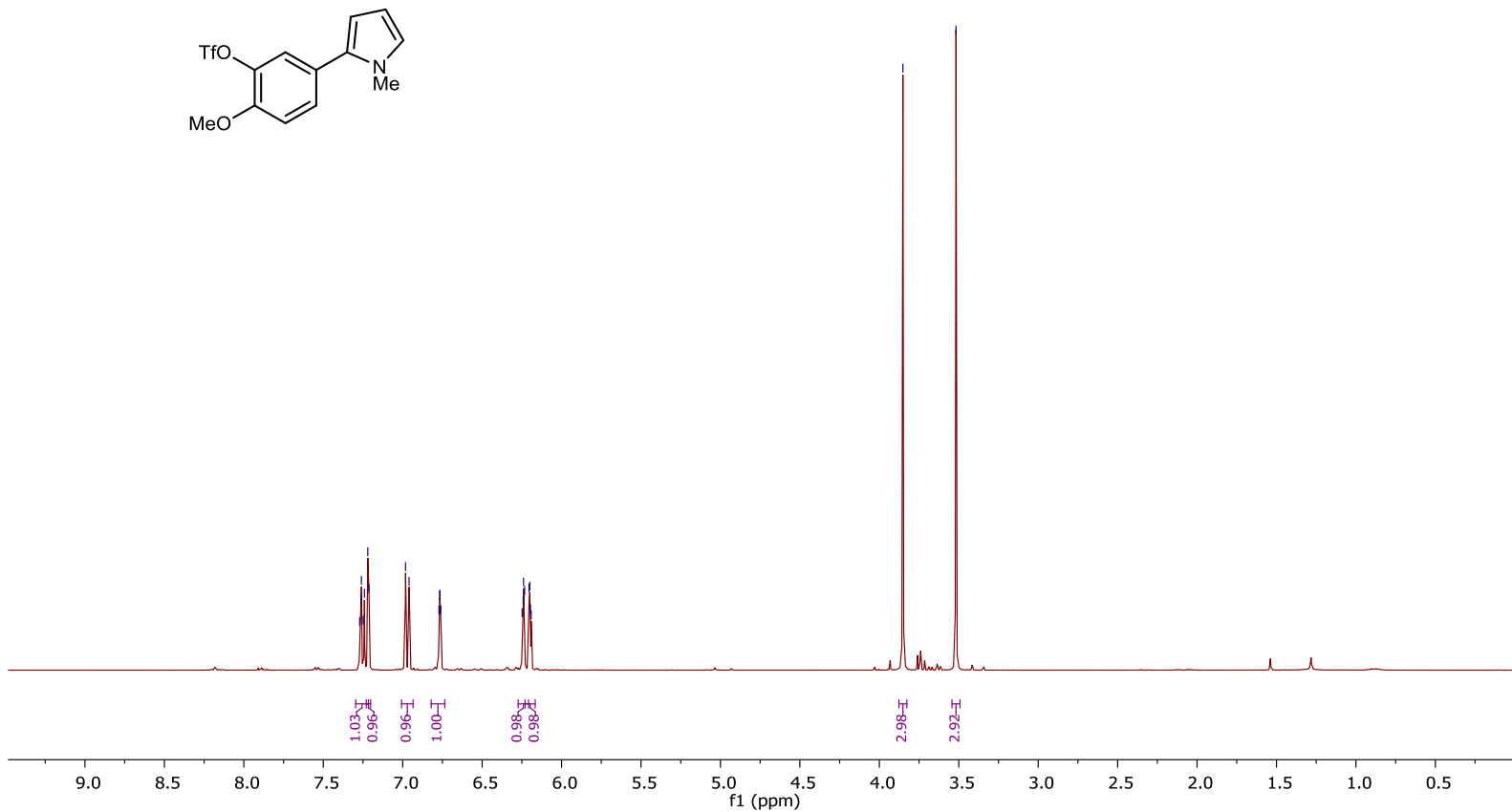

$22-{ }^{13} \mathrm{C}$ NMR (100 MHz, $\mathrm{CDCl}_{3}$ )

|<smiles>COc1ccc(-c2cccn2C)cc1O</smiles>

$\mathrm{MeO}$

$$
\text { Me }
$$


$22-{ }^{19} \mathrm{~F}$ NMR (376 MHz, $\mathrm{CDCl}_{3}$ )

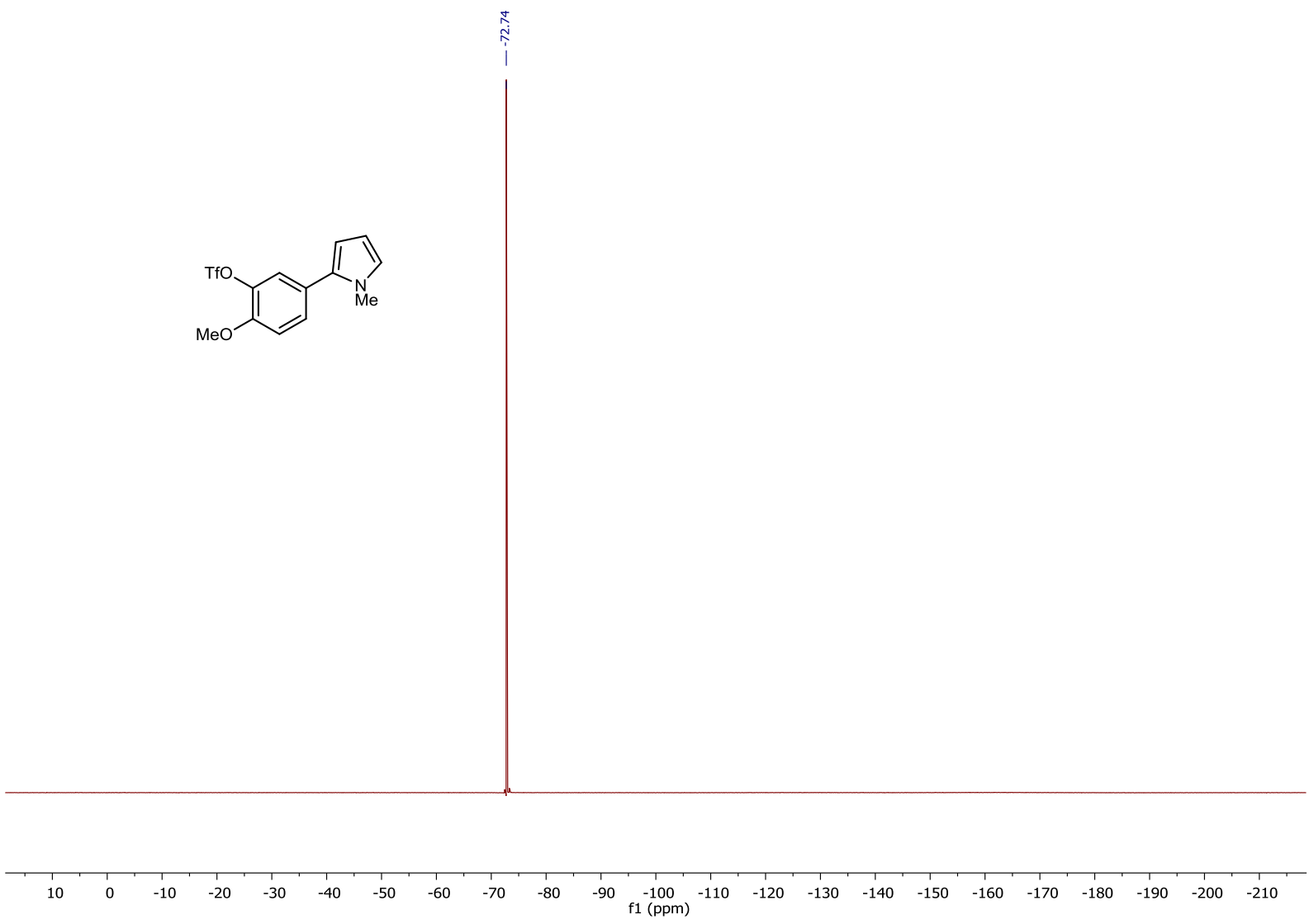


$23-{ }^{1} \mathrm{H}$ NMR $\left(400 \mathrm{MHz}, \mathrm{CDCl}_{3}\right)$

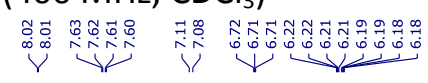

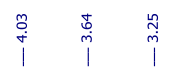
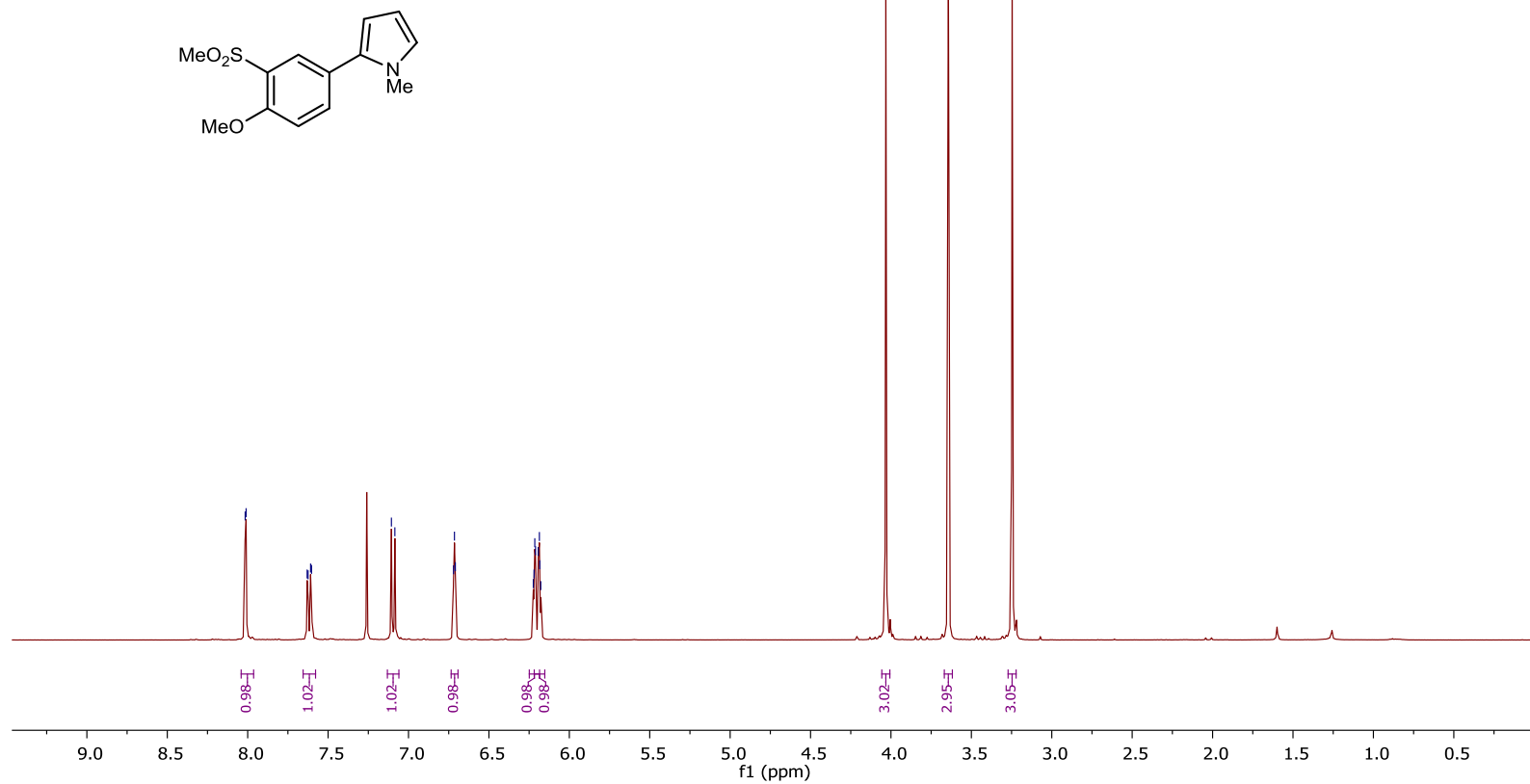

$23-{ }^{13} \mathrm{C}$ NMR $\left(100 \mathrm{MHz}, \mathrm{CDCl}_{3}\right)$

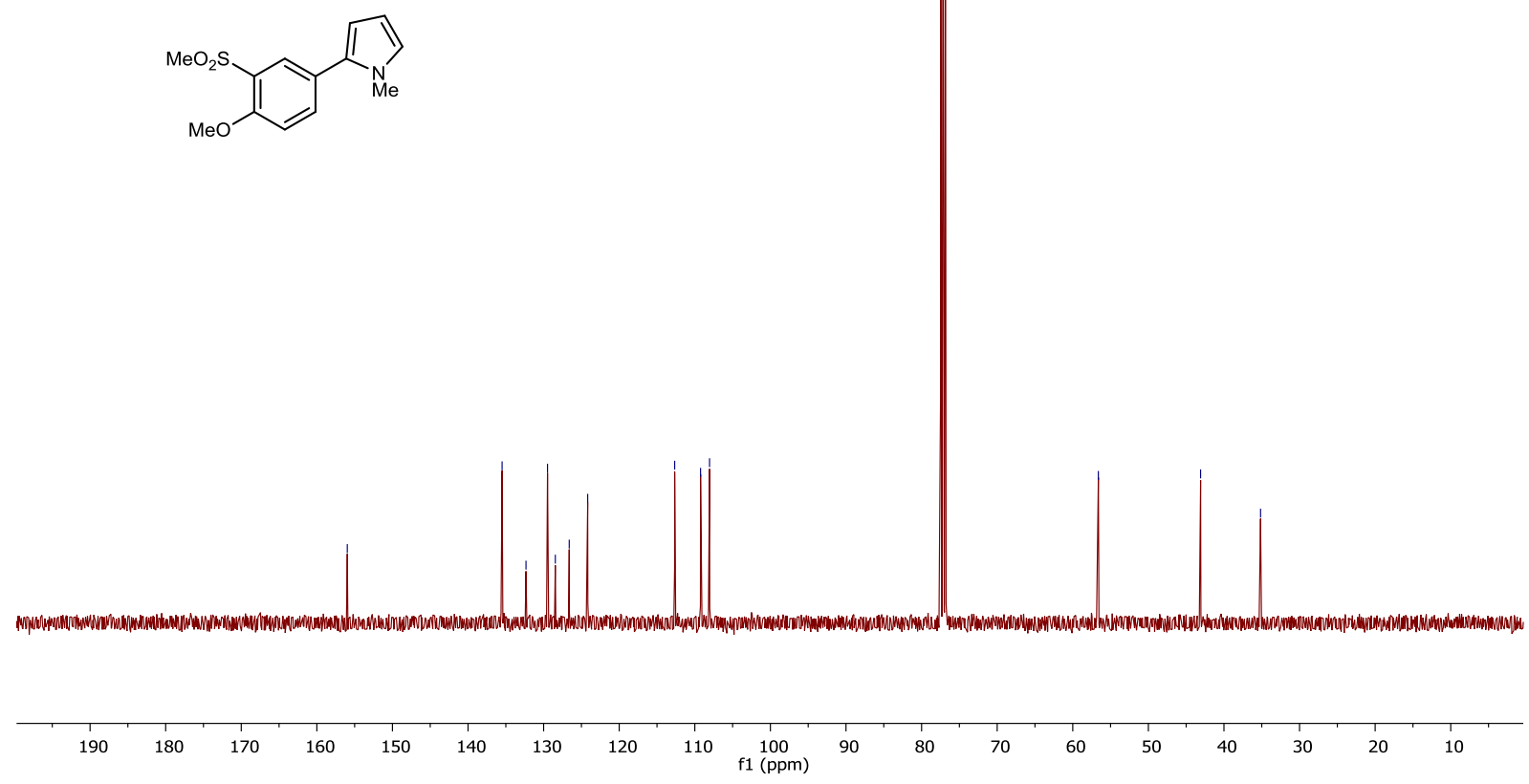

88 
$24-{ }^{1} \mathrm{H}$ NMR (400 MHz, $\mathrm{CDCl}_{3}$ )

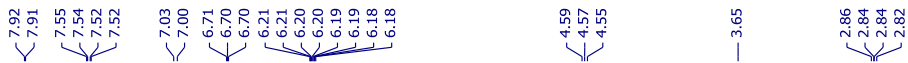<smiles></smiles>

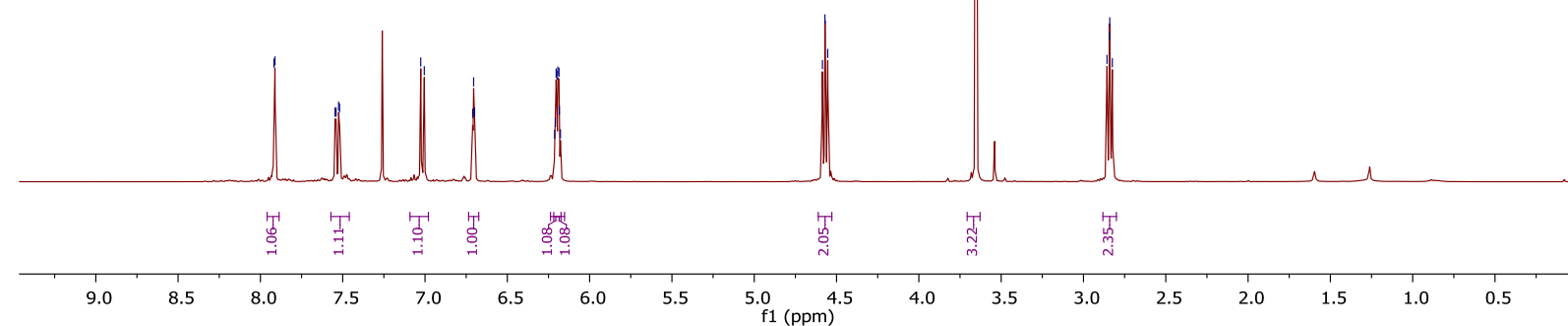

$24-{ }^{13} \mathrm{CNMR}\left(100 \mathrm{MHz}, \mathrm{CDCl}_{3}\right)$

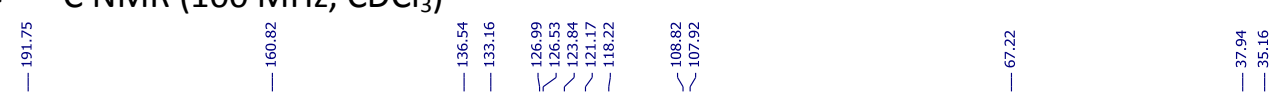<smiles></smiles>

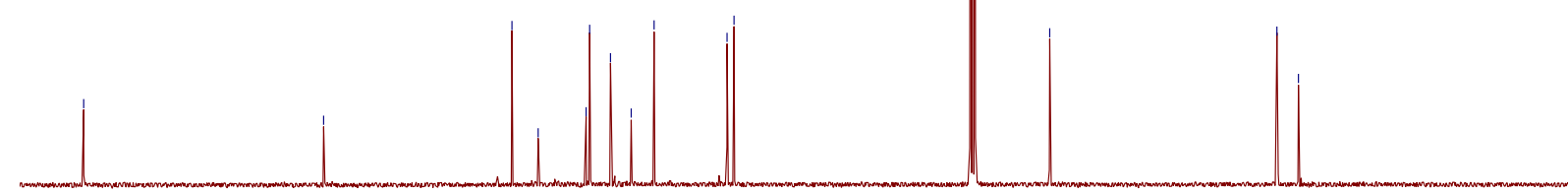

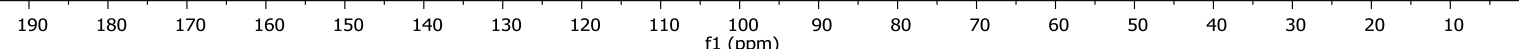


$25-{ }^{1} \mathrm{H}$ NMR $\left(400 \mathrm{MHz}, \mathrm{CDCl}_{3}\right)$

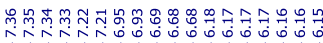

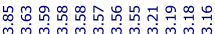

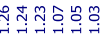

$\forall \forall$ Y<smiles>CCCCNC(=O)c1cc(-c2cccn2C)ccc1OC</smiles>

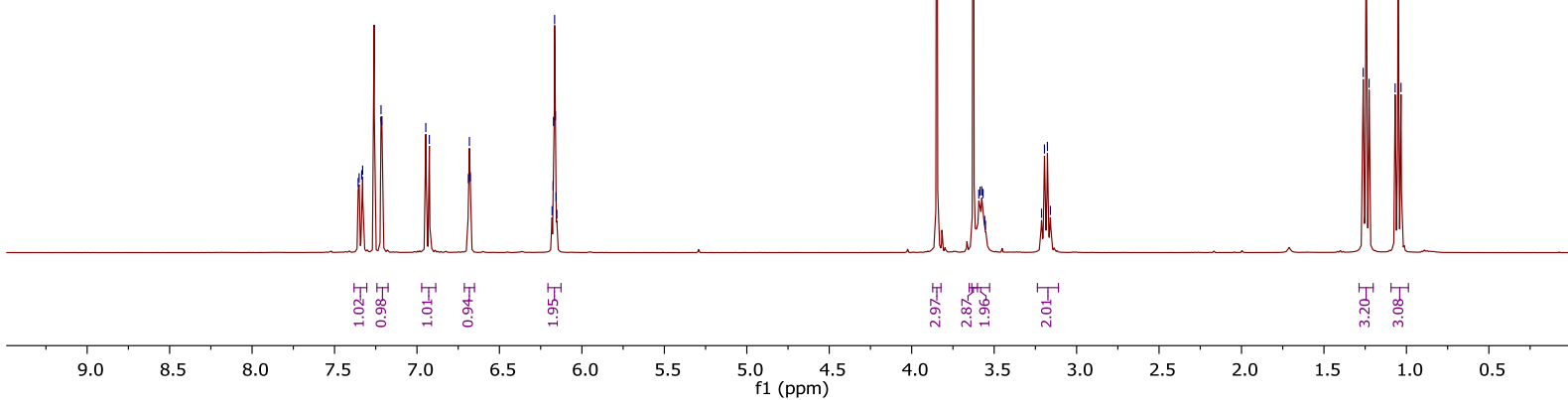

$25-{ }^{13} \mathrm{C}$ NMR $\left(100 \mathrm{MHz}, \mathrm{CDCl}_{3}\right)$

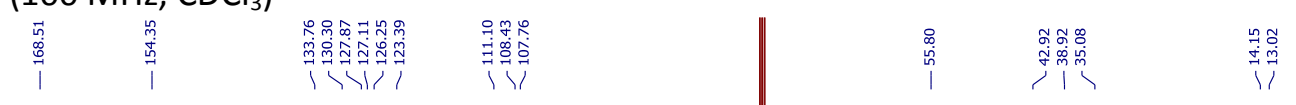<smiles>COc1ccc(-c2cccn2C)cc1C(=O)NCc1ccccc1</smiles>

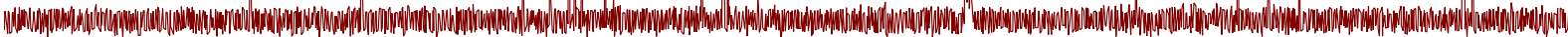
190
$180 \quad 170 \quad 160$
140
$\begin{array}{llr}120 & 110 & 100 \\ & \mathrm{f} 1(\mathrm{ppm})\end{array}$
$70 \quad 60$
50 


\section{$26-{ }^{1} \mathrm{H}$ NMR $\left(400 \mathrm{MHz}, \mathrm{CDCl}_{3}\right)$}

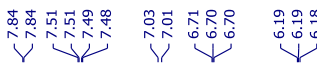

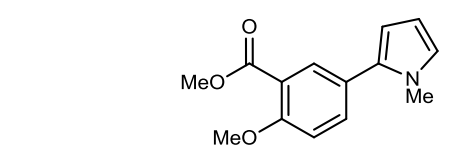

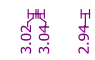

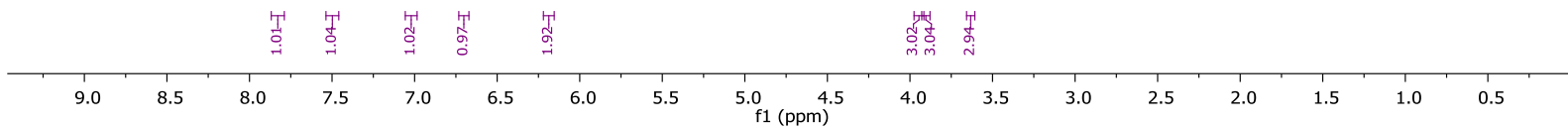

$26-{ }^{13} \mathrm{C} \mathrm{NMR}\left(100 \mathrm{MHz}, \mathrm{CDCl}_{3}\right)$<smiles></smiles>

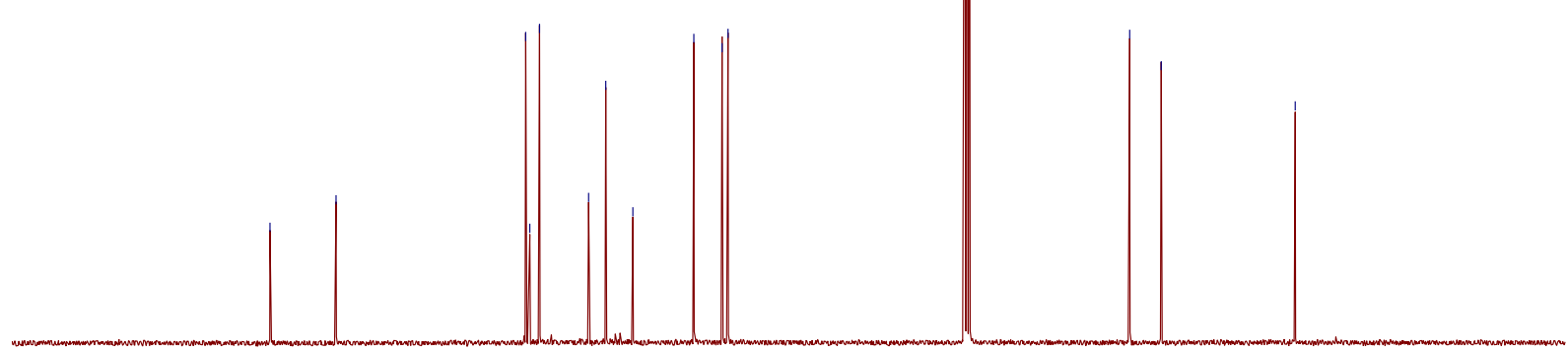

$\begin{array}{llllllllll}190 & 180 & 170 & 160 & 150 & 140 & 130 & 120 & 110 & \begin{array}{c}100 \\ \mathrm{f} 1(\mathrm{ppm})\end{array}\end{array}$ 
$27-{ }^{1} \mathrm{H}$ NMR $\left(400 \mathrm{MHz}, \mathrm{CDCl}_{3}\right)$

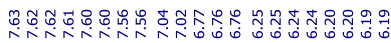

$\stackrel{\substack{\infty \\ i}}{\stackrel{0}{m}}$<smiles></smiles>

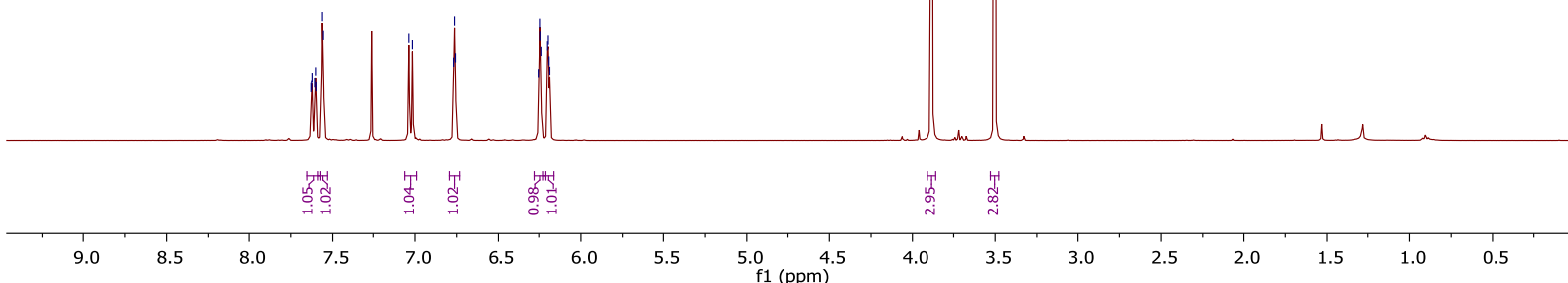

$27-{ }^{13} \mathrm{C}$ NMR $\left(100 \mathrm{MHz}, \mathrm{CDCl}_{3}\right)$

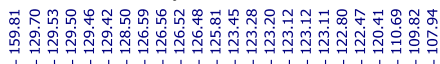<smiles></smiles>

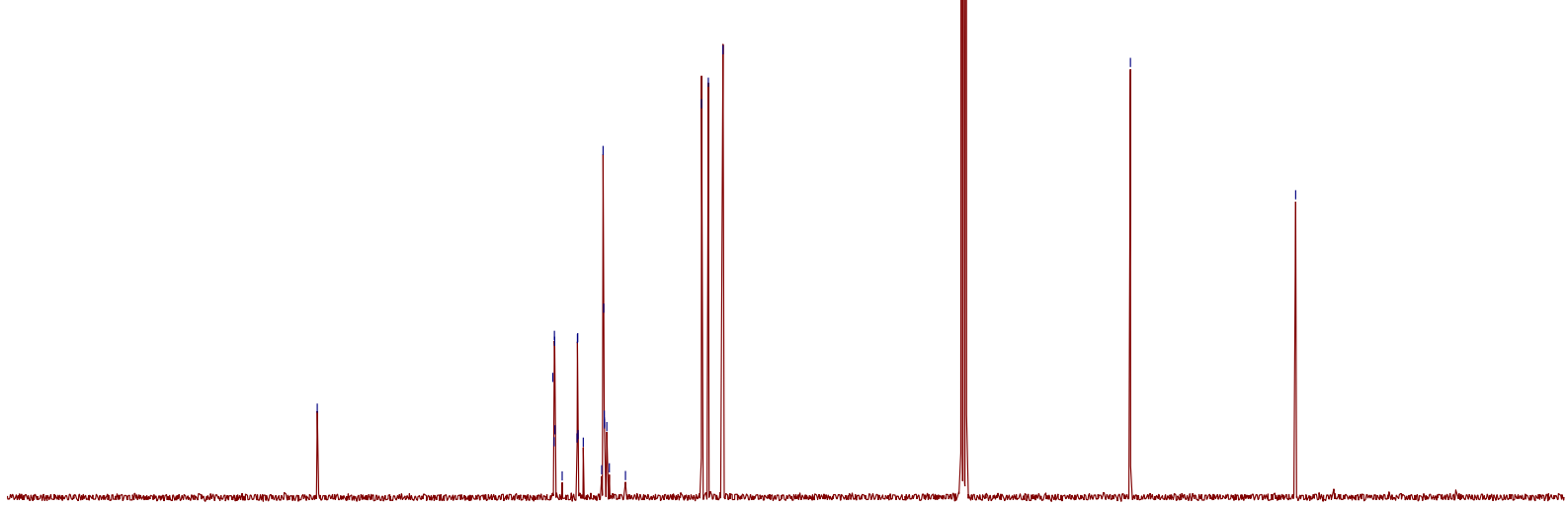

$190 \quad 180$

$160 \quad 150$

140

120

$\stackrel{100}{\mathrm{f} 1(\mathrm{ppm})}$

$90 \quad 80$

70

50

10 
$27-{ }^{19} \mathrm{~F}$ NMR $\left(376 \mathrm{MHz}, \mathrm{CDCl}_{3}\right)$

ịุ<smiles></smiles>

$\begin{array}{llllllllllllllllllllllllllllll}10 & 0 & -10 & -20 & -30 & -40 & -50 & -60 & -70 & -80 & -90 & -100 & -110 & -120 & -130 & -140 & -150 & -160 & -170 & -180 & -190 & -200 & -210\end{array}$ 
$28-{ }^{1} \mathrm{H}$ NMR $\left(400 \mathrm{MHz}, \mathrm{CDCl}_{3}\right)$

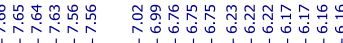<smiles></smiles>

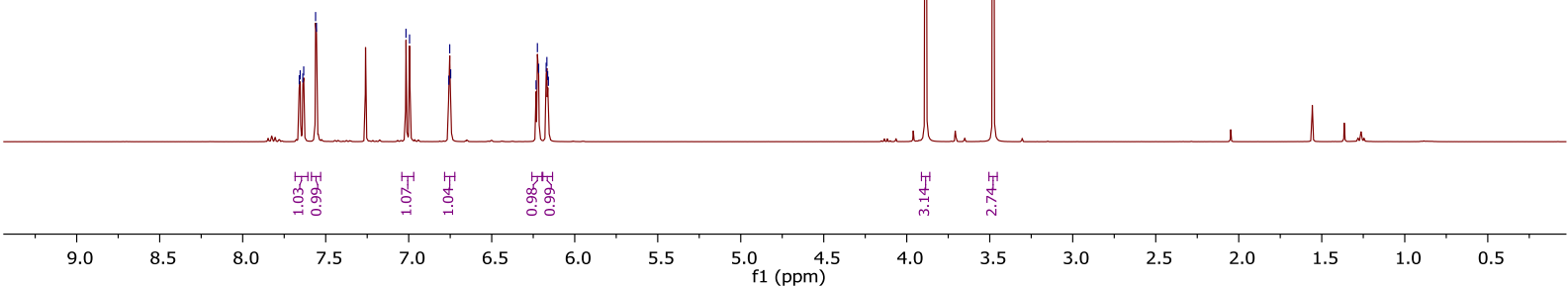

$28-{ }^{13} \mathrm{C}$ NMR (100 MHz, $\left.\mathrm{CDCl}_{3}\right)$

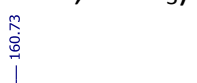

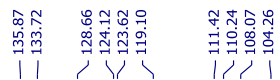

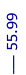<smiles></smiles>

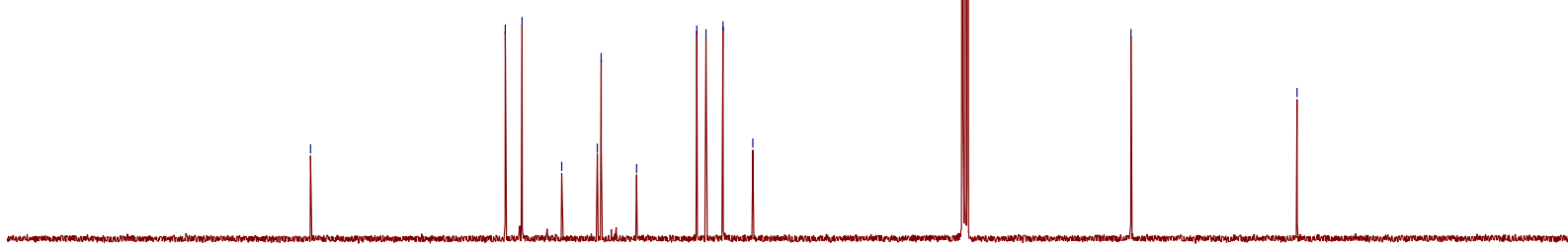

$\begin{array}{lllll}190 & 180 & 170 & 160 & 150\end{array}$

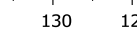

$\begin{array}{ccc}110 & 100 \\ \mathrm{f} 1(\mathrm{ppm}) & 90\end{array}$

$80 \quad 70$

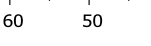

$30 \quad 20 \quad 10$ 
$29-{ }^{1} \mathrm{H}$ NMR $\left(400 \mathrm{MHz}, \mathrm{CDCl}_{3}\right)$

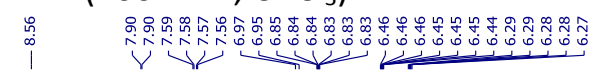

空怘
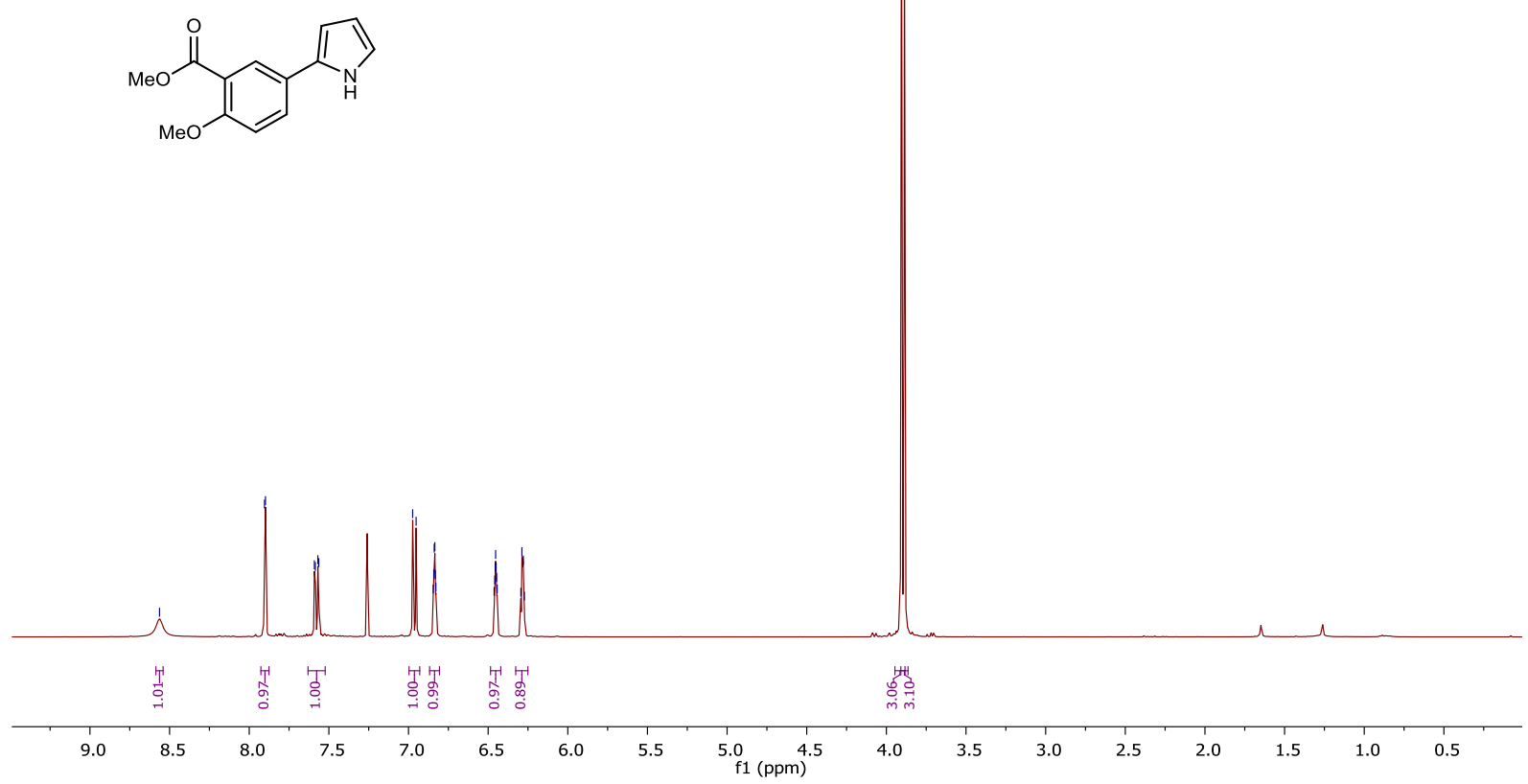

$29-{ }^{13} \mathrm{C}$ NMR $\left(100 \mathrm{MHz}, \mathrm{CDCl}_{3}\right)$

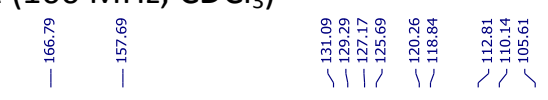

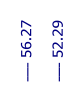
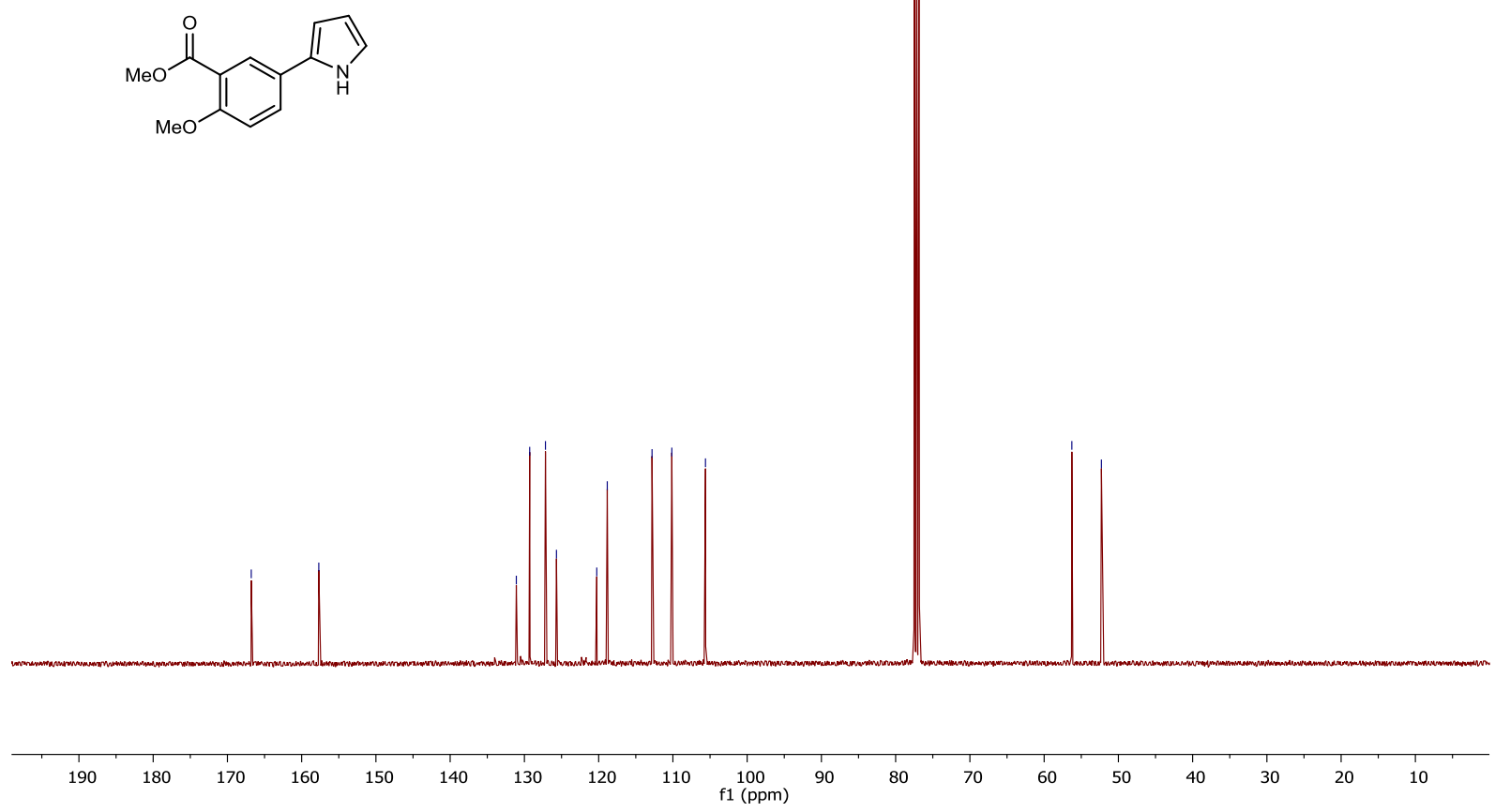

95 
$30-{ }^{1} \mathrm{H}$ NMR $\left(400 \mathrm{MHz}, \mathrm{CDCl}_{3}\right)$

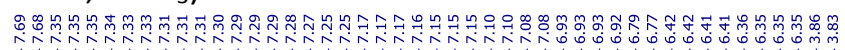
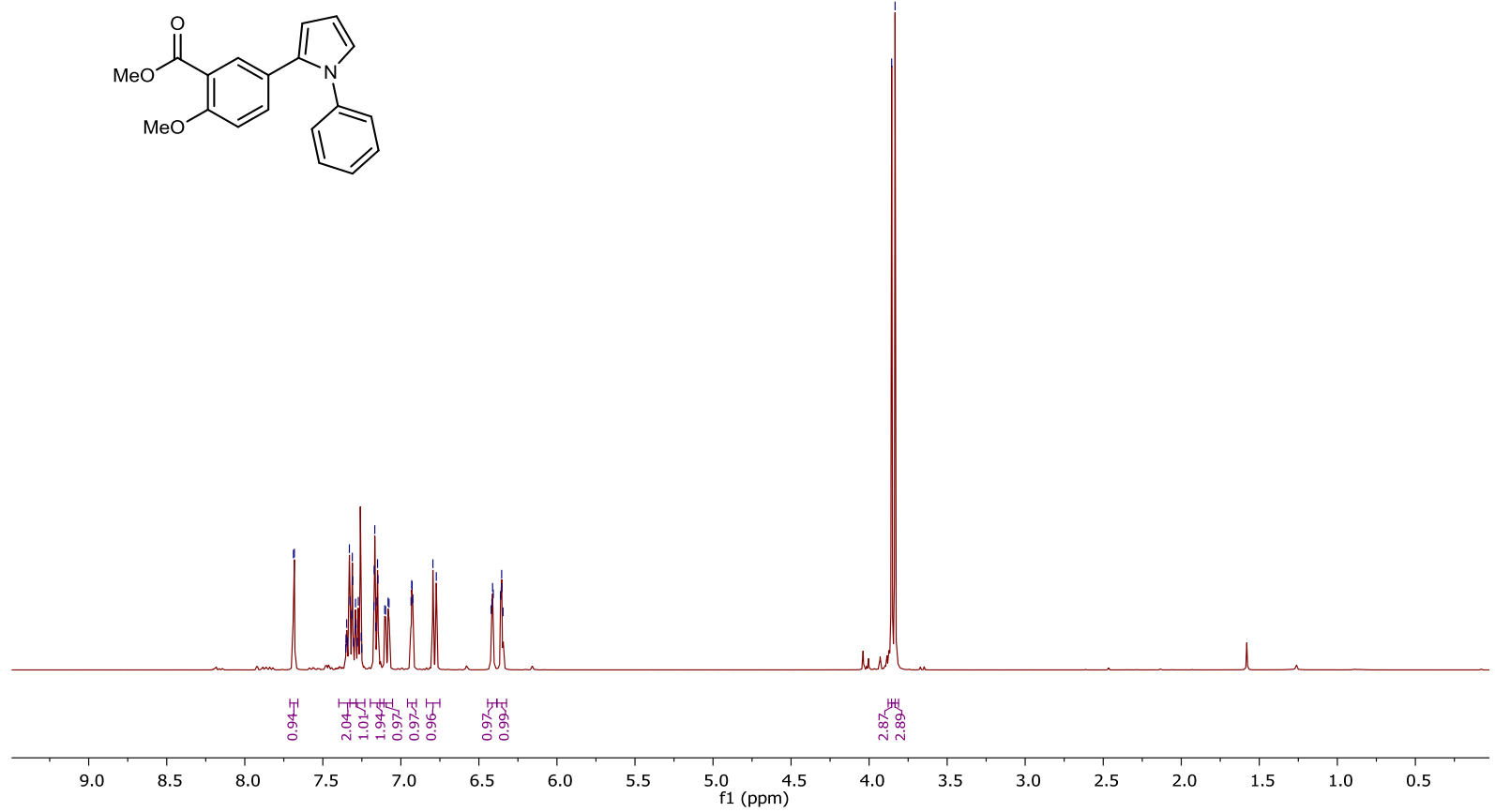

$30-{ }^{13} \mathrm{CNMR}\left(100 \mathrm{MHz}, \mathrm{CDCl}_{3}\right)$

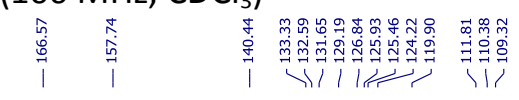

暲
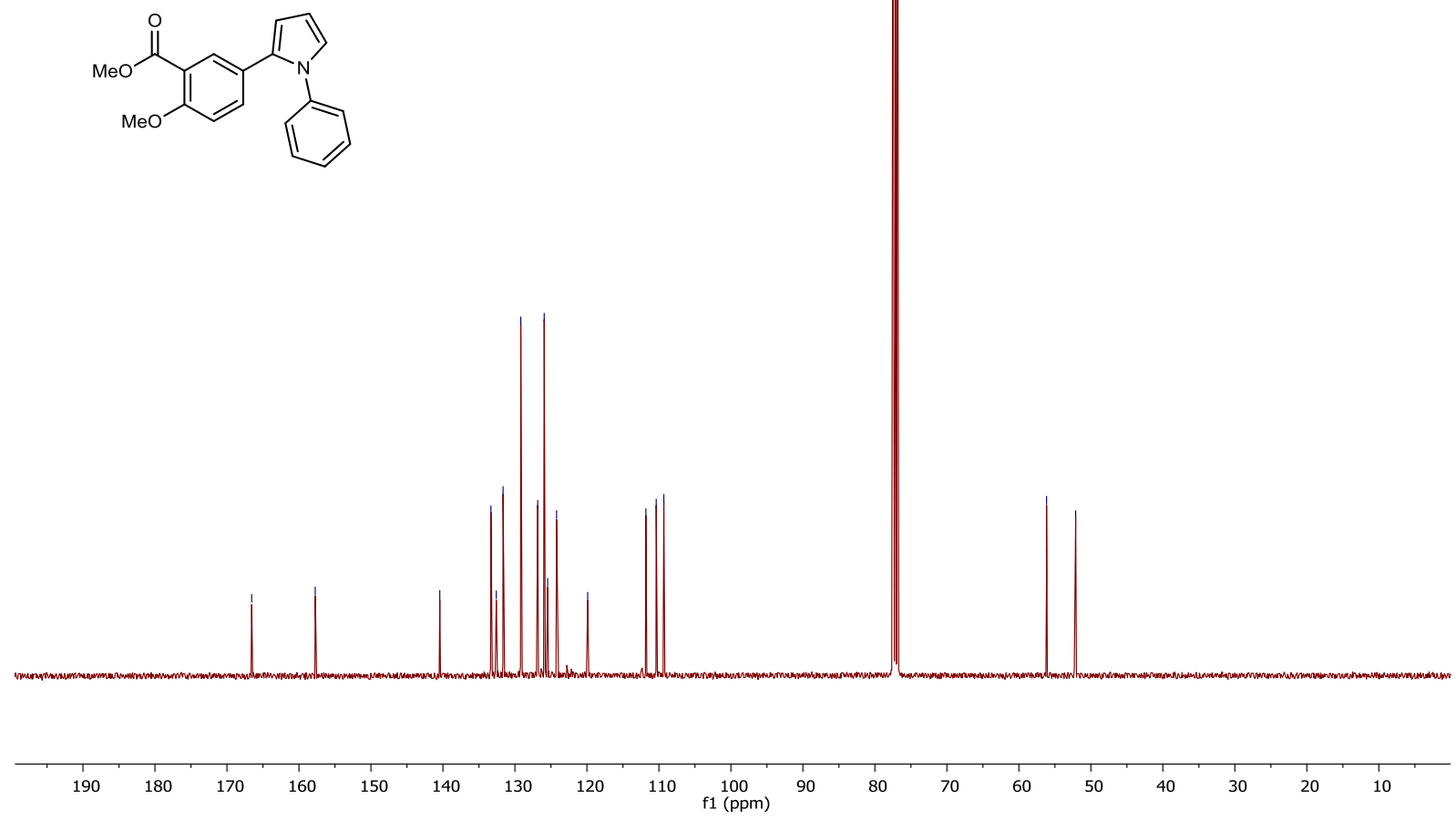

96 
$31-{ }^{1} \mathrm{H}$ NMR $\left(400 \mathrm{MHz}, \mathrm{CDCl}_{3}\right)$

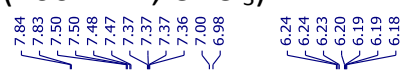

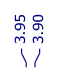

$\stackrel{\substack{1 \\ i}}{i}$
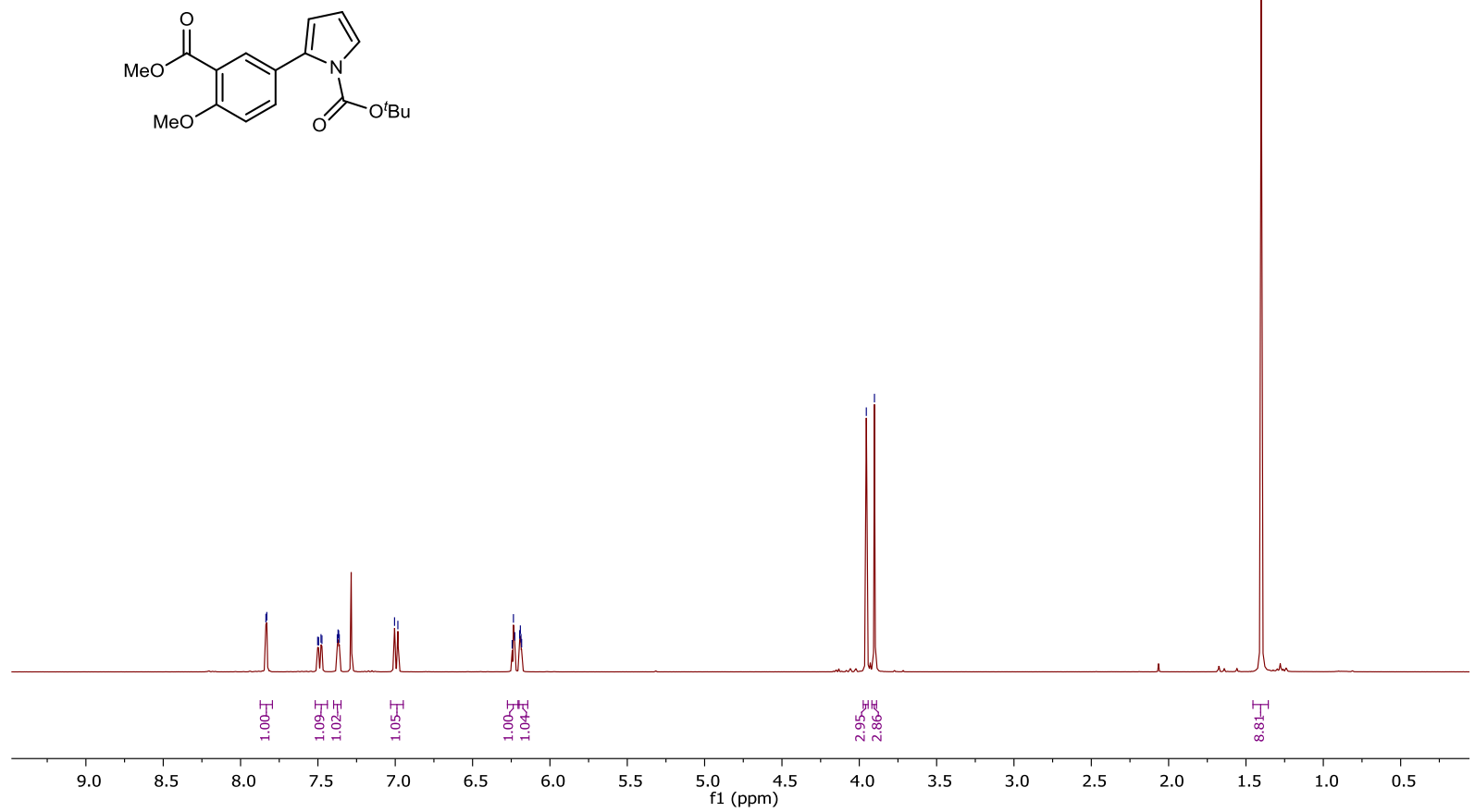

$31-{ }^{13} \mathrm{C}$ NMR $\left(100 \mathrm{MHz}, \mathrm{CDCl}_{3}\right)$

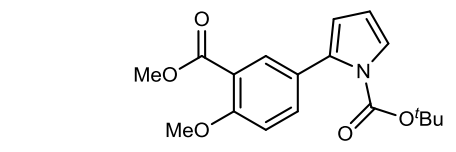

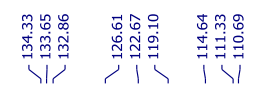

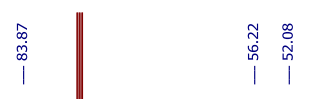

$\stackrel{\infty}{i}$

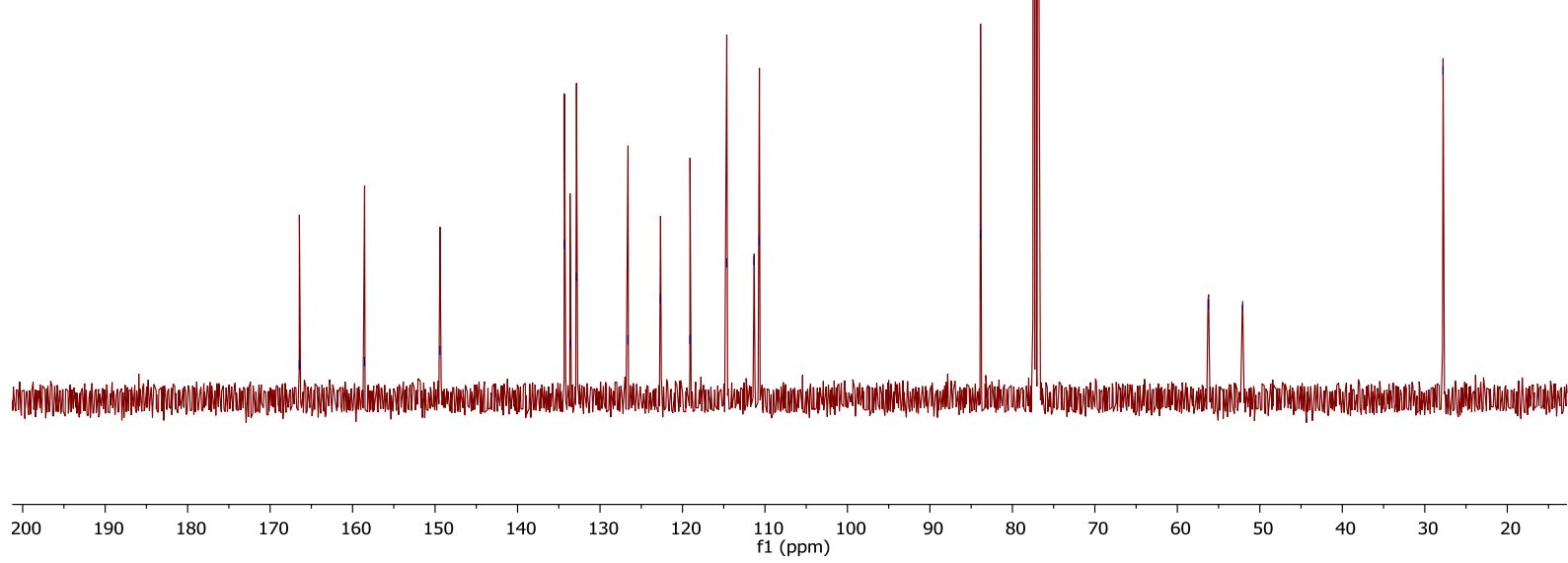

97 
$2-{ }^{1} \mathrm{H}$ NMR (400 MHz, $\left.\mathrm{CDCl}_{3}\right)$

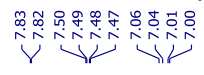

ㄱํำ

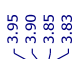

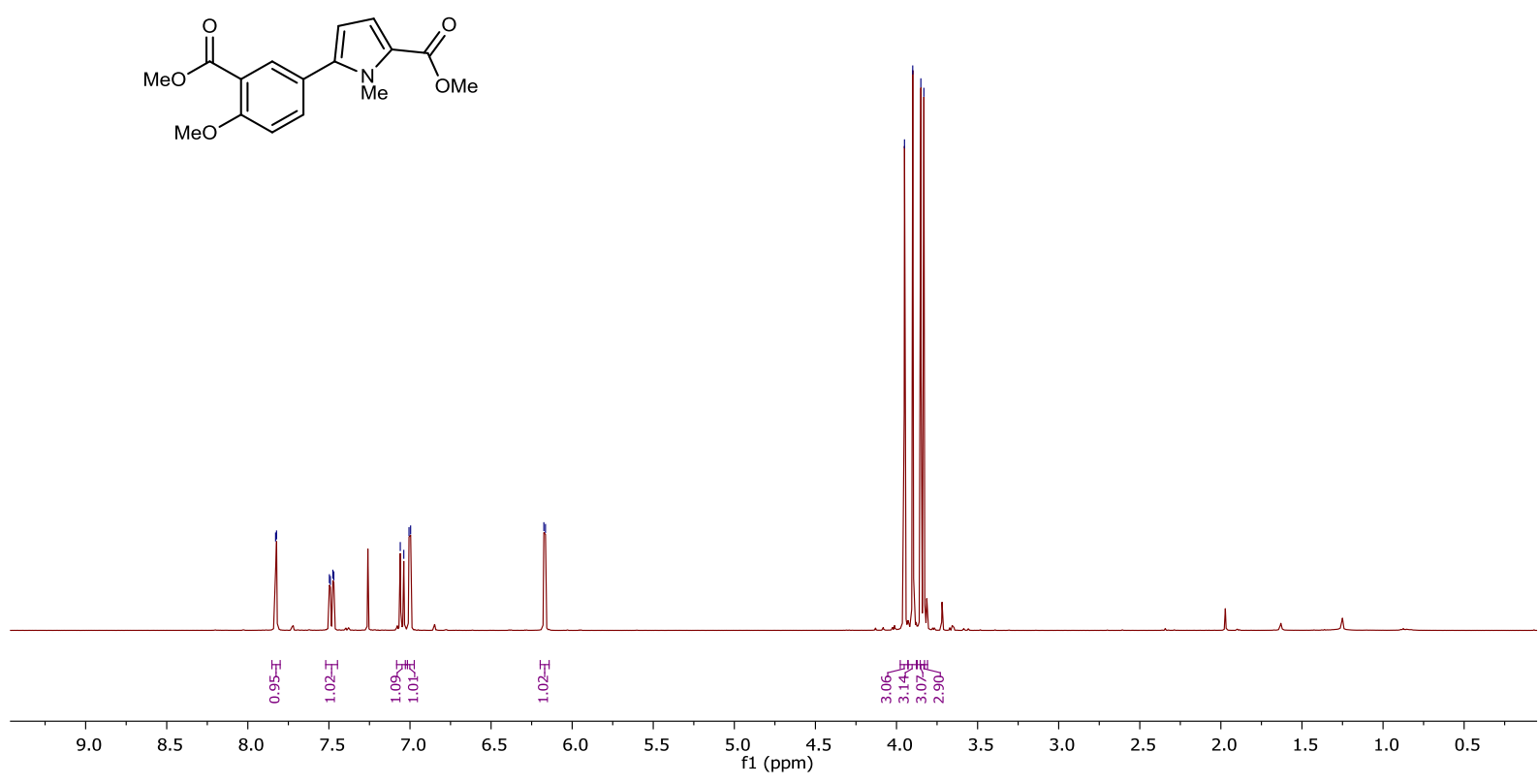

$32-{ }^{13} \mathrm{C}$ NMR $\left(100 \mathrm{MHz}, \mathrm{CDCl}_{3}\right)$

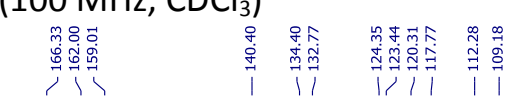

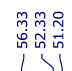
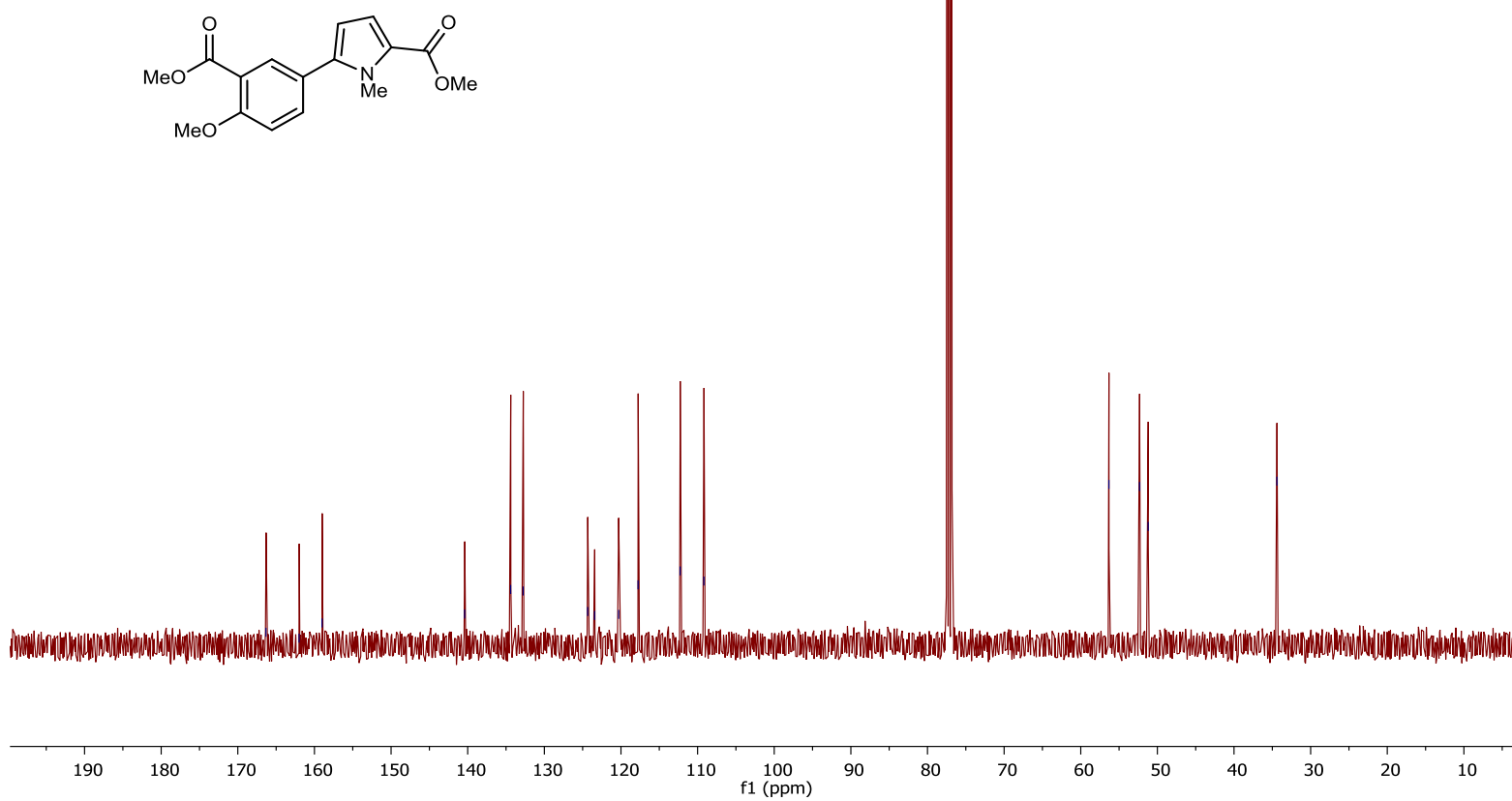

98 
$33-{ }^{1} \mathrm{H}$ NMR $\left(400 \mathrm{MHz}, \mathrm{CDCl}_{3}\right)$

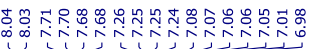<smiles>COC(=O)c1cc(-c2cccs2)ccc1OC</smiles>

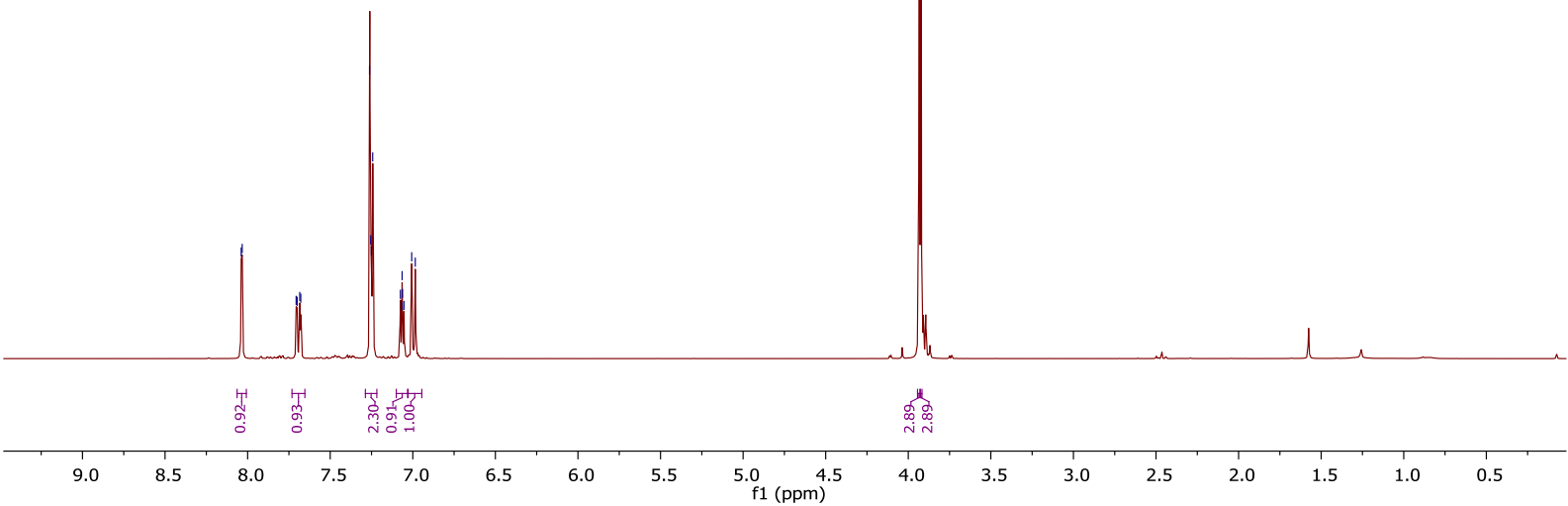

$33-{ }^{13} \mathrm{C}$ NMR $\left(100 \mathrm{MHz}, \mathrm{CDCl}_{3}\right)$

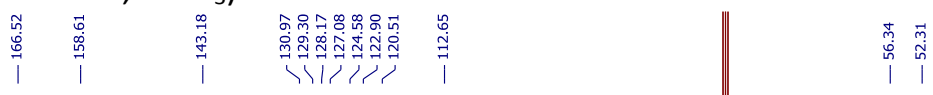<smiles>COc1ccc(-c2cccs2)cc1C(=O)O</smiles>

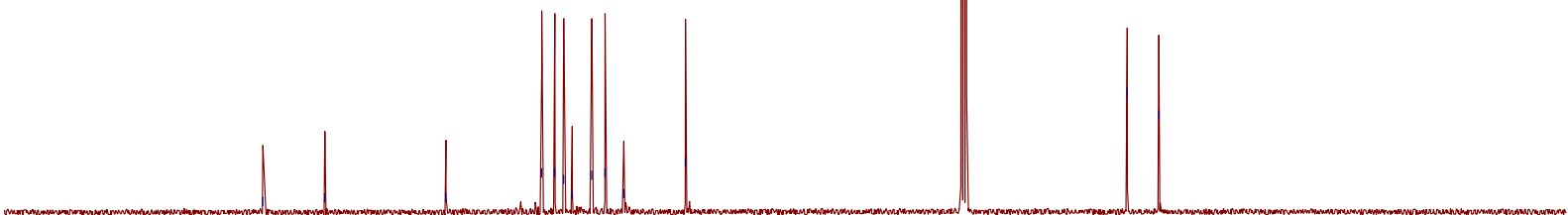

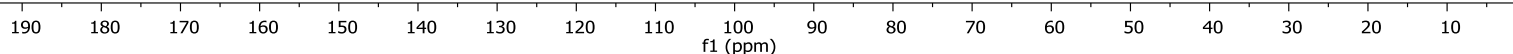


$34-{ }^{1} \mathrm{H}$ NMR (400 MHz, $\left.\mathrm{CDCl}_{3}\right)$

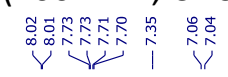

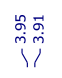
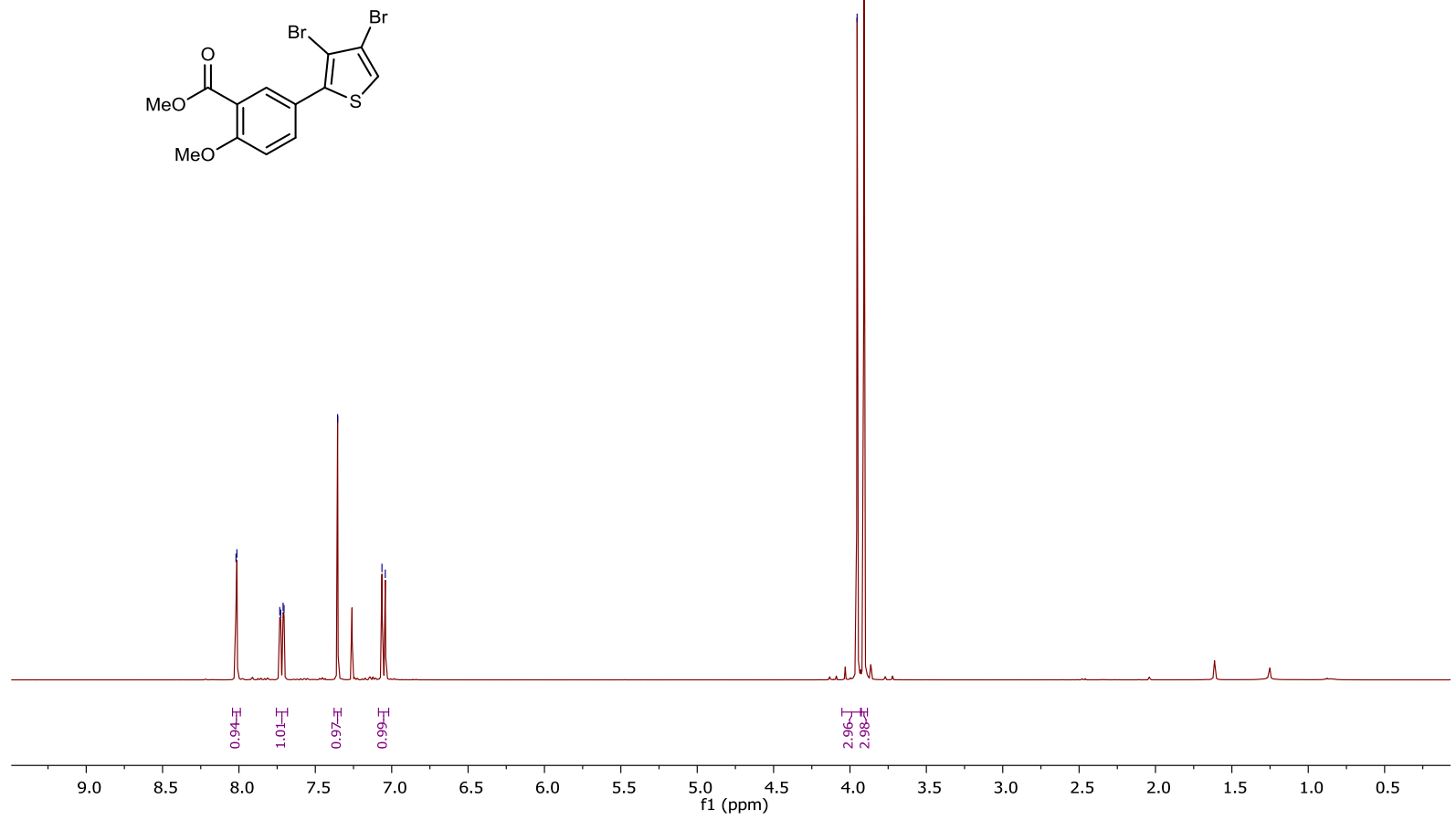

$34-{ }^{13} \mathrm{CNMR}\left(100 \mathrm{MHz}, \mathrm{CDCl}_{3}\right)$
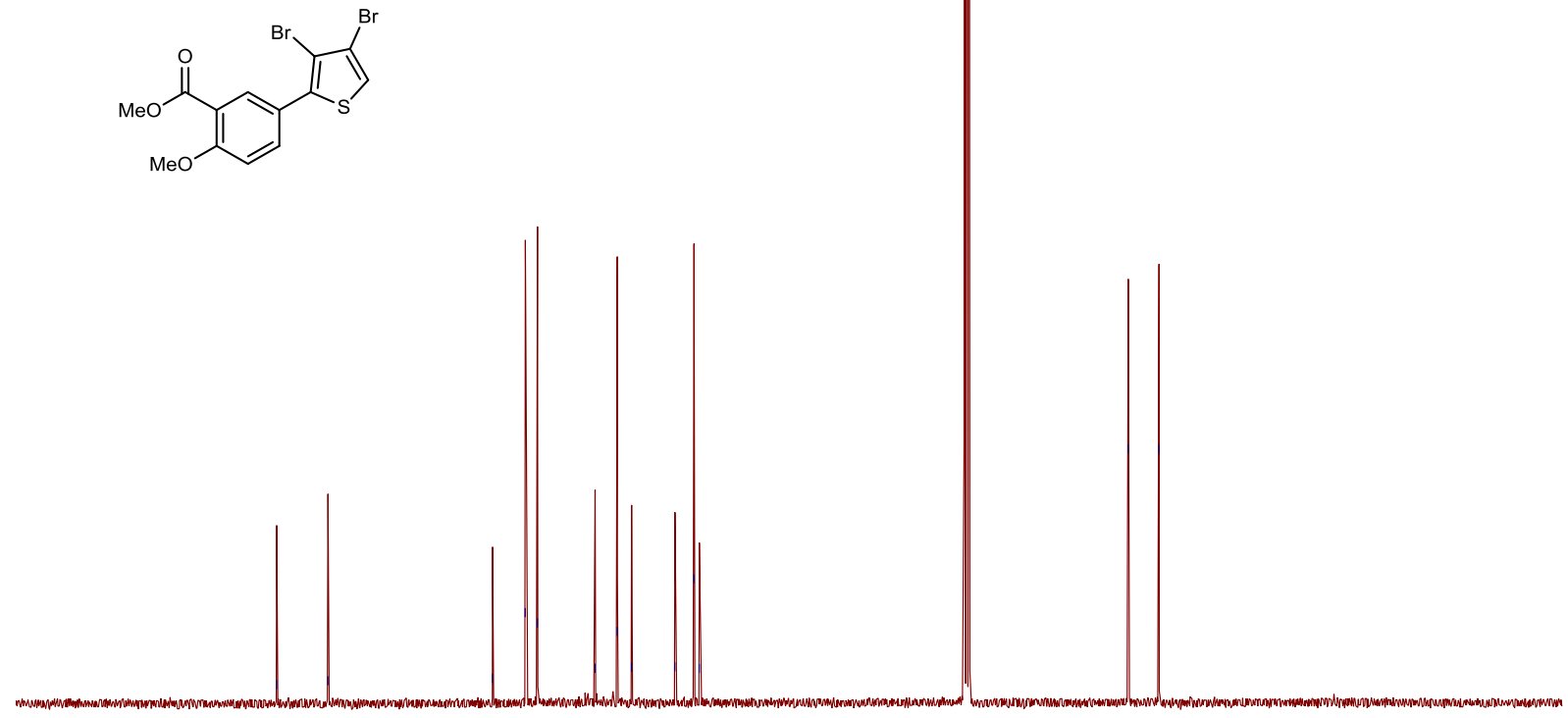

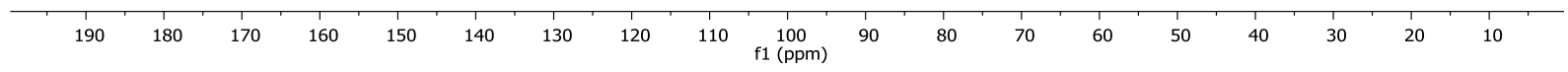

100 
$35-{ }^{1} \mathrm{H}$ NMR $\left(400 \mathrm{MHz}, \mathrm{CDCl}_{3}\right)$

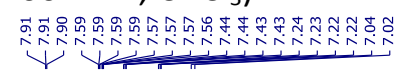

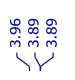

บับ
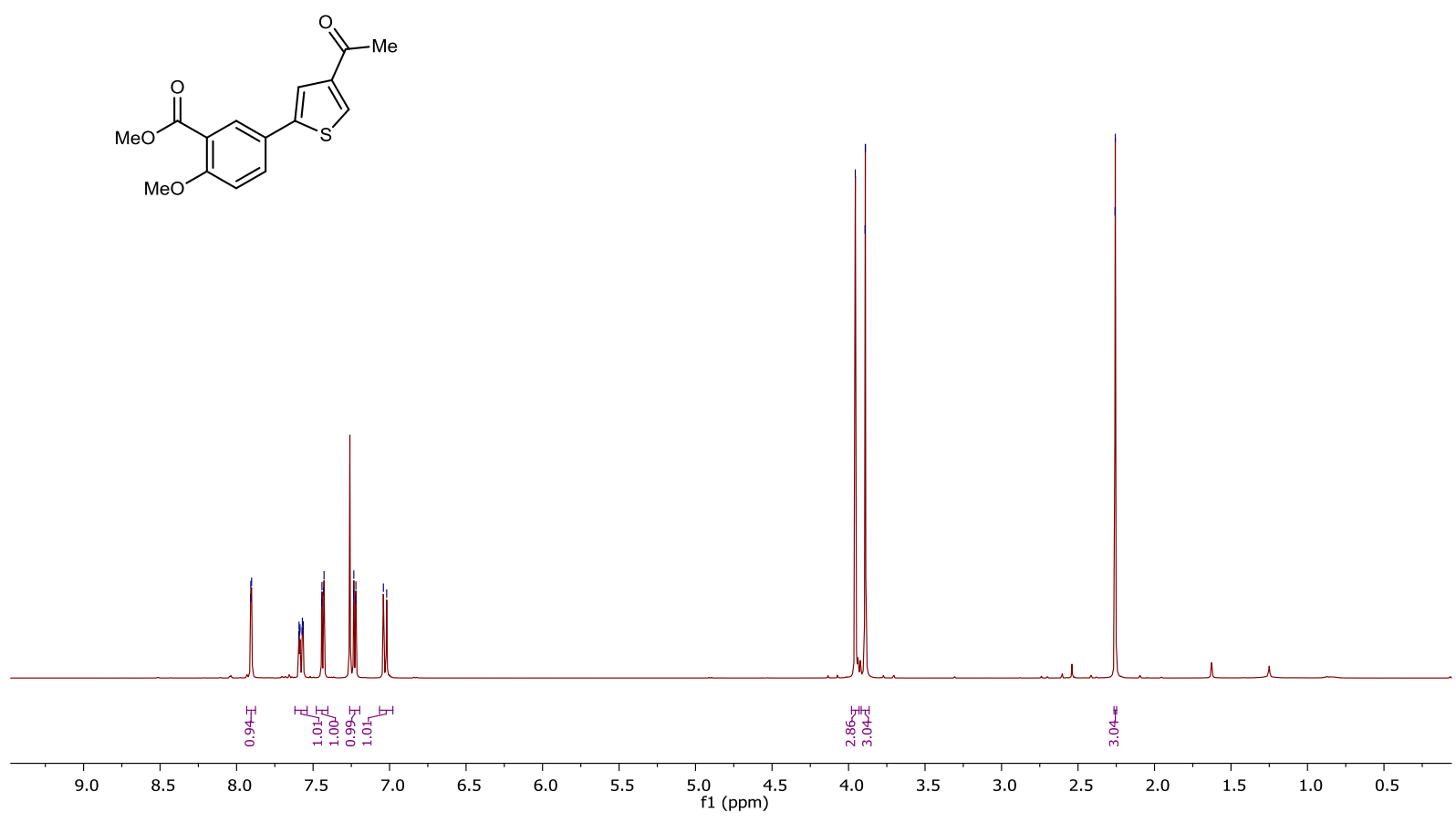

$35-{ }^{13} \mathrm{C}$ NMR $\left(100 \mathrm{MHz}, \mathrm{CDCl}_{3}\right)$

II II
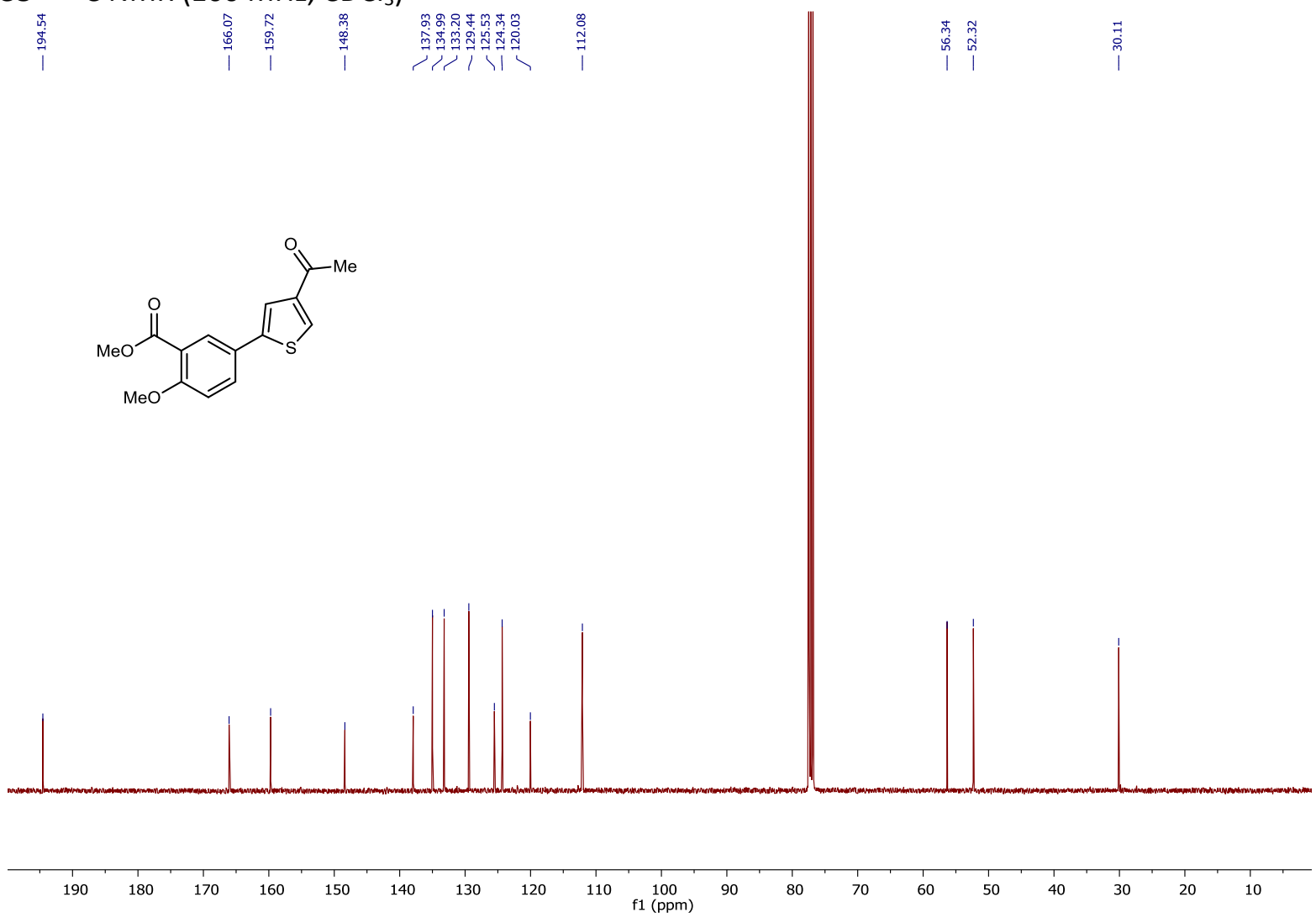

101 
$36-{ }^{1} \mathrm{H}$ NMR $\left(400 \mathrm{MHz}, \mathrm{CDCl}_{3}\right)$

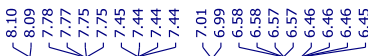<smiles>COc1ccc(-c2ccco2)cc1C(=O)O</smiles>

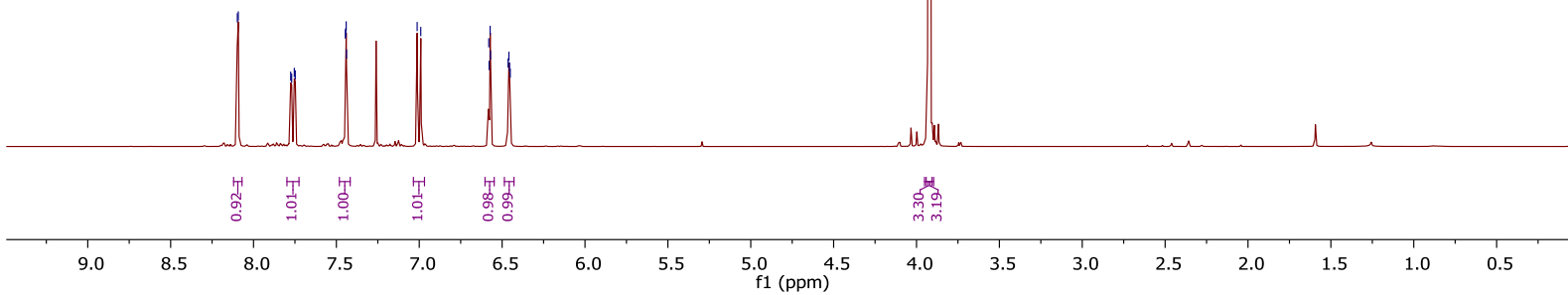

$36-{ }^{13} \mathrm{C}$ NMR $\left(100 \mathrm{MHz}, \mathrm{CDCl}_{3}\right)$

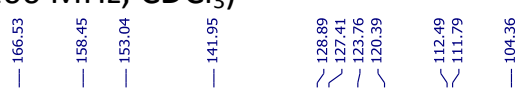<smiles>COc1ccc(-c2ccco2)cc1C(=O)O</smiles>

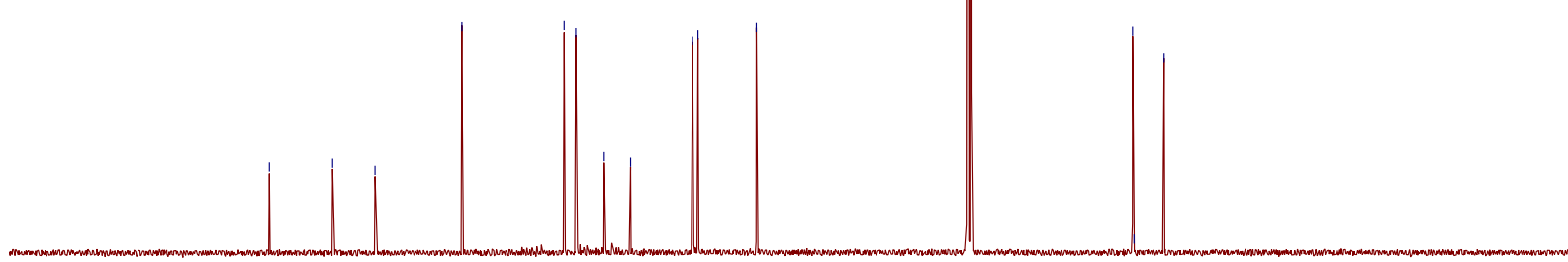

$\begin{array}{llllllllll}190 & 180 & 170 & 160 & 150 & 140 & 130 & 120 & 110 & \begin{array}{c}100 \\ \mathrm{f} 1(\mathrm{ppm})\end{array}\end{array}$

80 50 
$37-{ }^{1} \mathrm{H}$ NMR $\left(500 \mathrm{MHz}, \mathrm{CDCl}_{3}\right)$

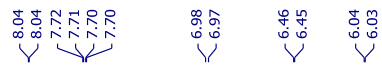<smiles>COc1ccc(-c2ccc(C)o2)cc1C(=O)O</smiles>

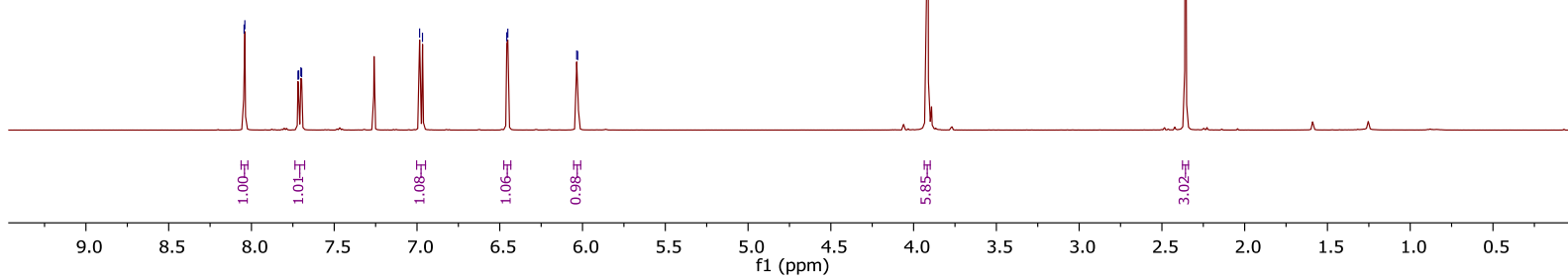

$37-{ }^{13} \mathrm{C}$ NMR (125 MHz, $\mathrm{CDCl}_{3}$ )

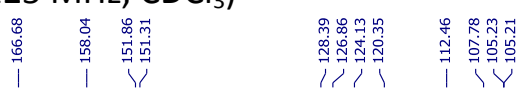<smiles>COc1ccc(-c2ccc(C)o2)cc1C(=O)O</smiles>

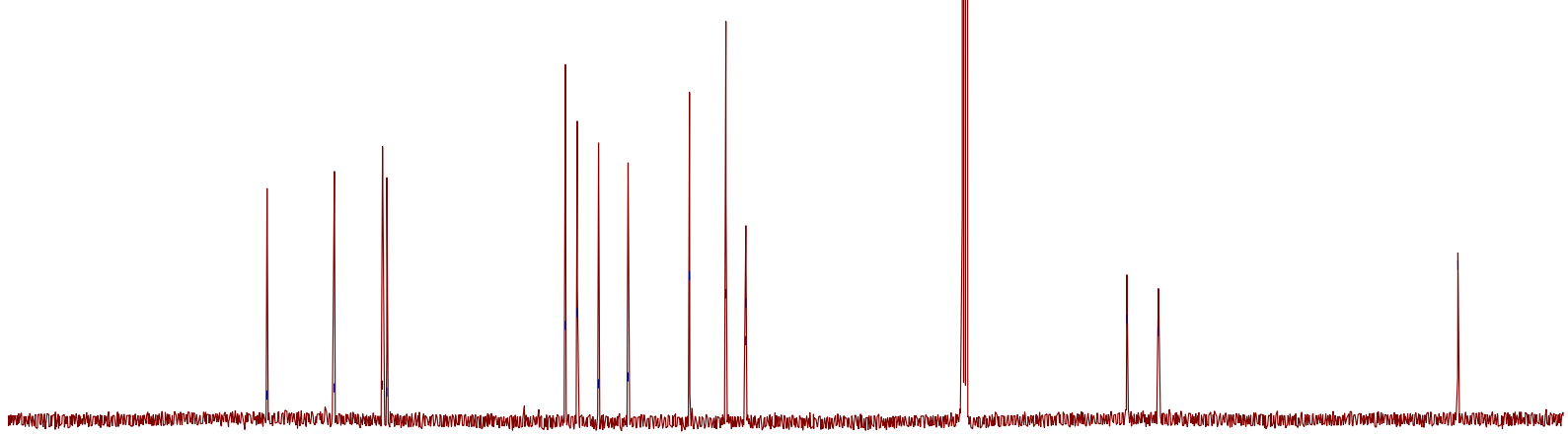


$38-{ }^{1} \mathrm{H}$ NMR $\left(400 \mathrm{MHz}, \mathrm{CDCl}_{3}\right)$

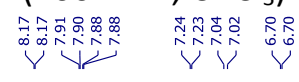

垫第

$\stackrel{\vec{n}}{1}$
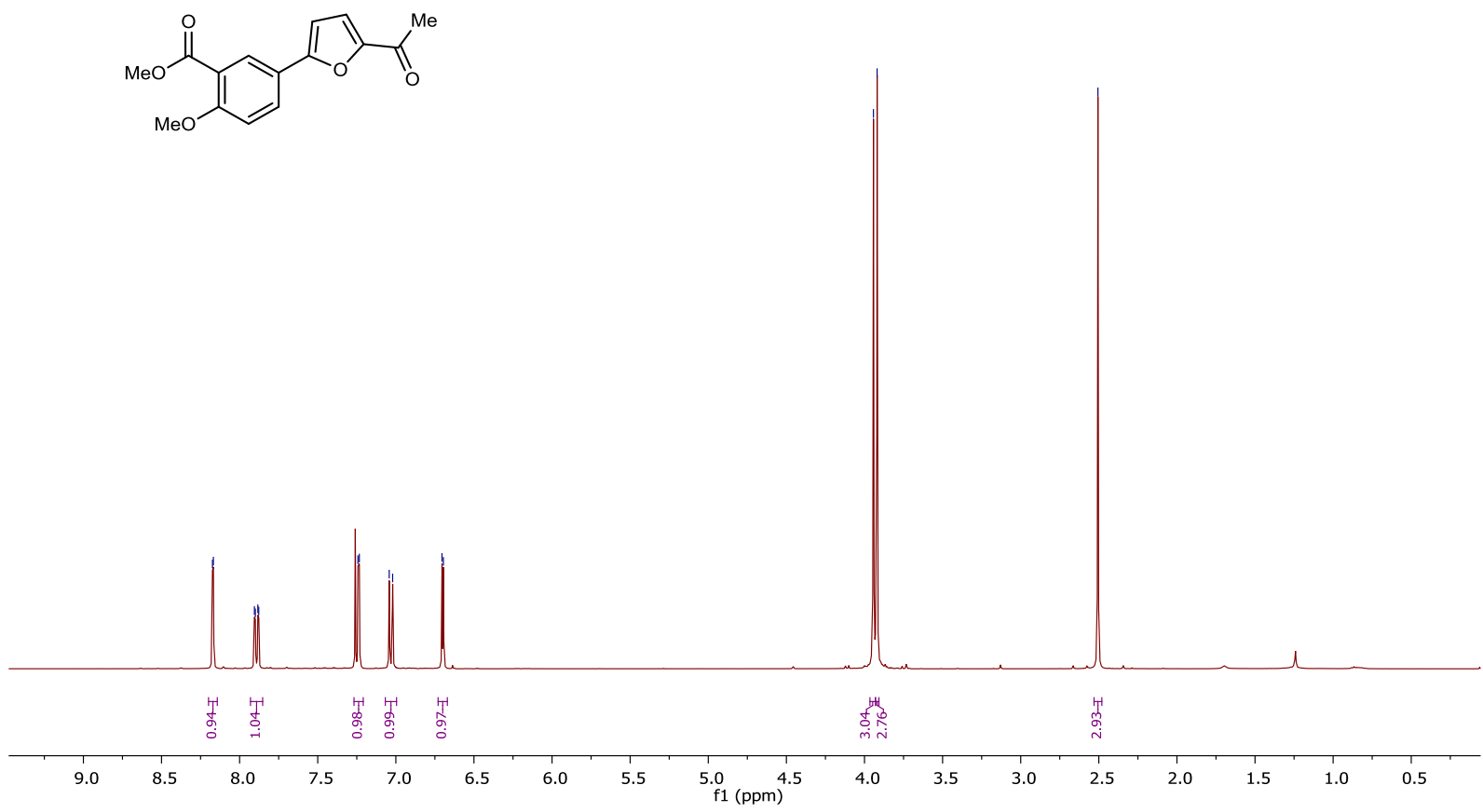

$38-{ }^{13} \mathrm{C}$ NMR (100 MHz, $\left.\mathrm{CDCl}_{3}\right)$

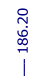

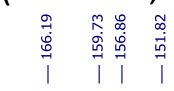

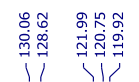

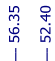

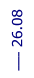
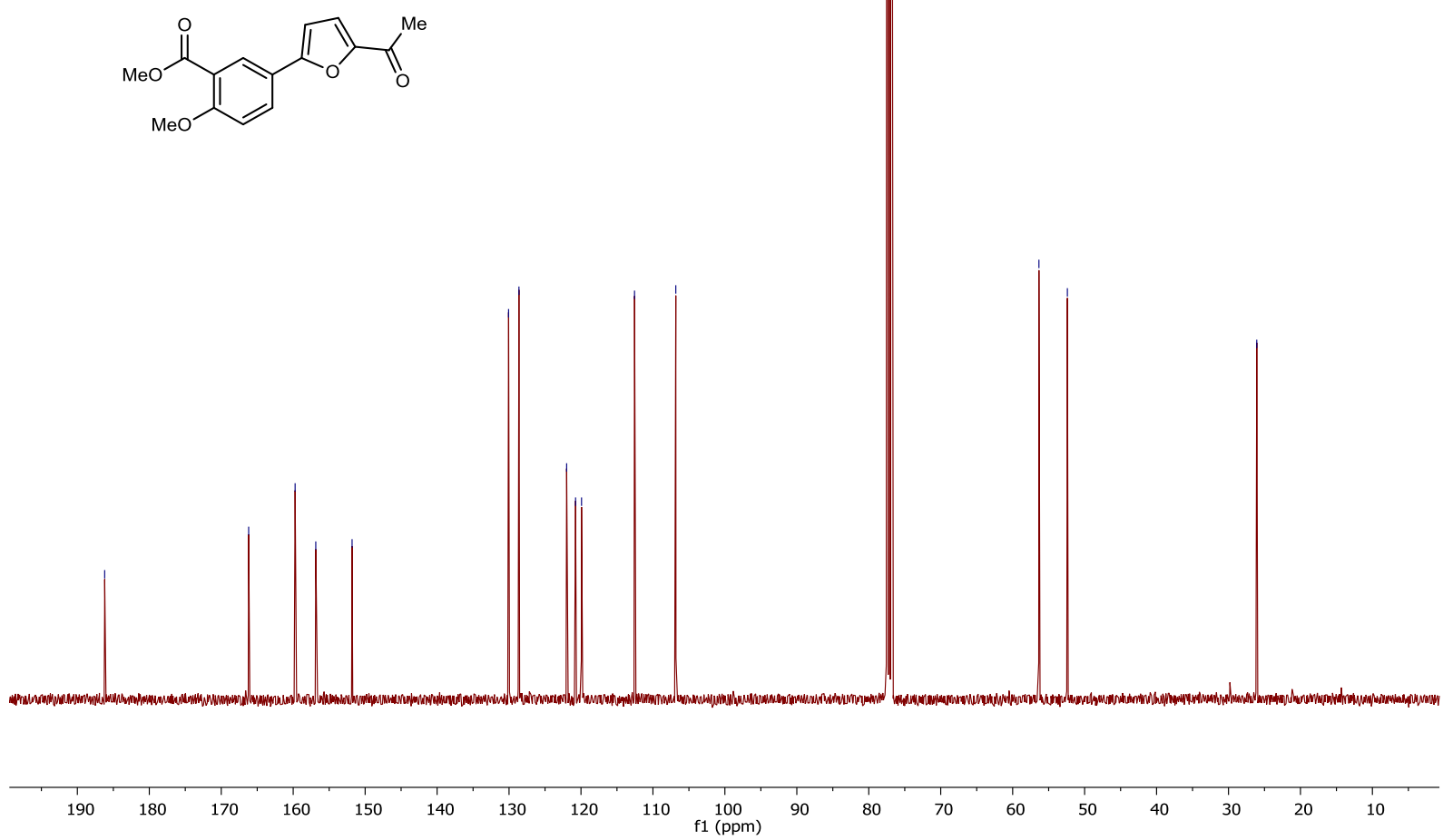

104 
$39-{ }^{1} \mathrm{H}$ NMR $\left(500 \mathrm{MHz}, \mathrm{CDCl}_{3}\right)$

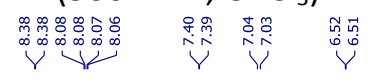

势第
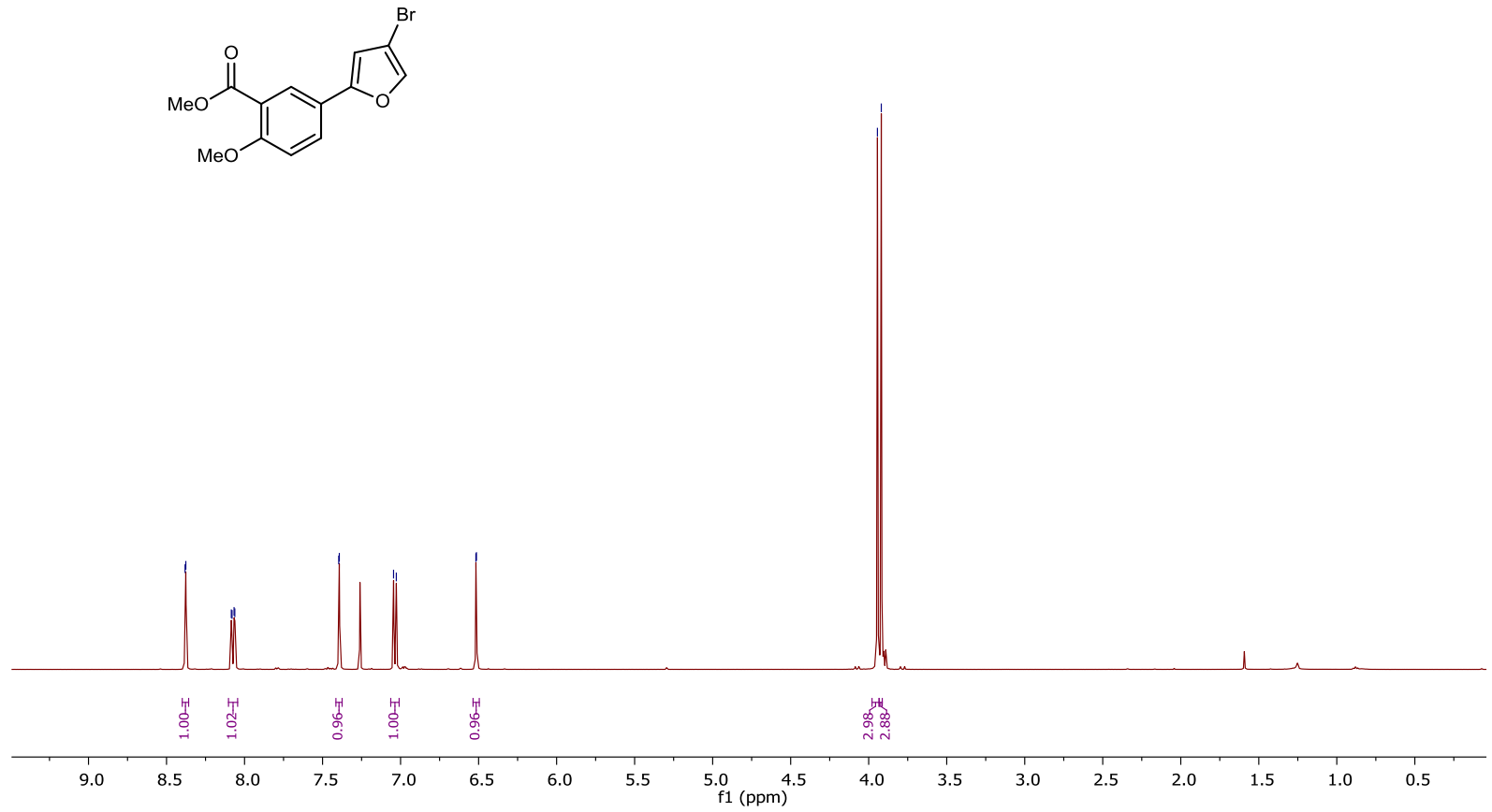

$39-{ }^{13} \mathrm{C}$ NMR (125 MHz, $\left.\mathrm{CDCl}_{3}\right)$

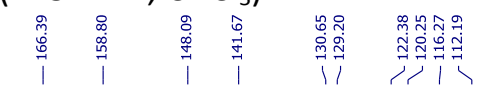
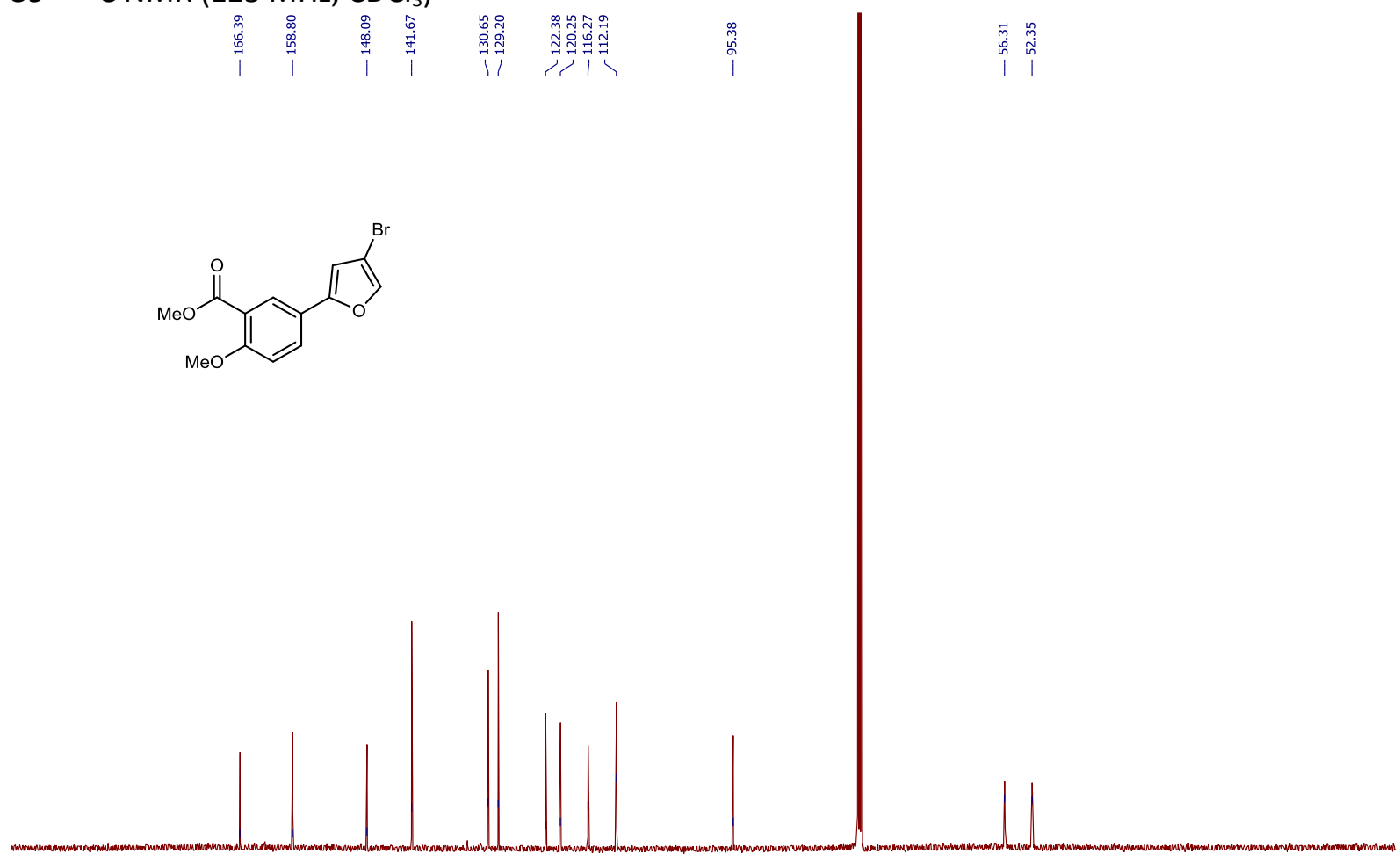

190

180

$170 \quad 160$

150

120

100
$\mathrm{f} 1(\mathrm{ppm})$

105 
$40-{ }^{1} \mathrm{H}$ NMR $\left(400 \mathrm{MHz}, \mathrm{CDCl}_{3}\right)$

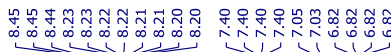<smiles>COC(=O)c1coc(-c2ccc(OC)c(C(=O)OC)c2)c1</smiles>

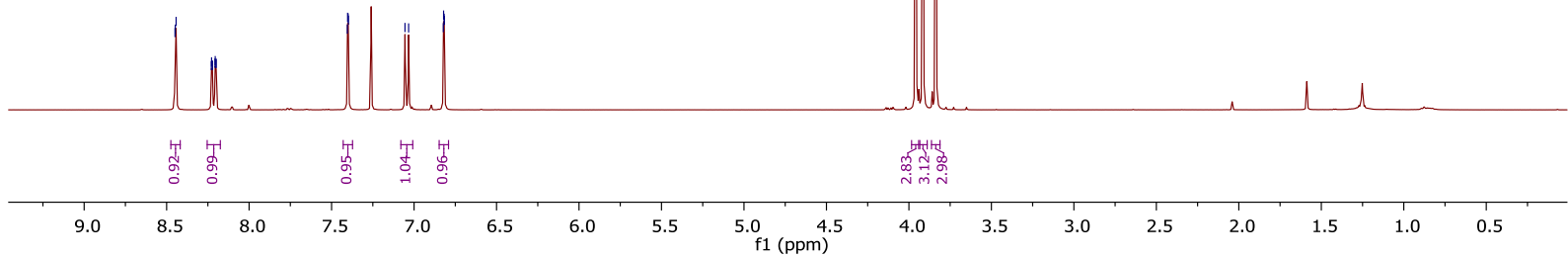

$40-{ }^{13} \mathrm{C}$ NMR $\left(100 \mathrm{MHz}, \mathrm{CDCl}_{3}\right)$

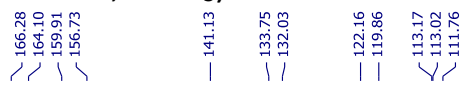<smiles>COC(=O)c1coc(-c2ccc(OC)c(C(=O)OC)c2)c1</smiles>

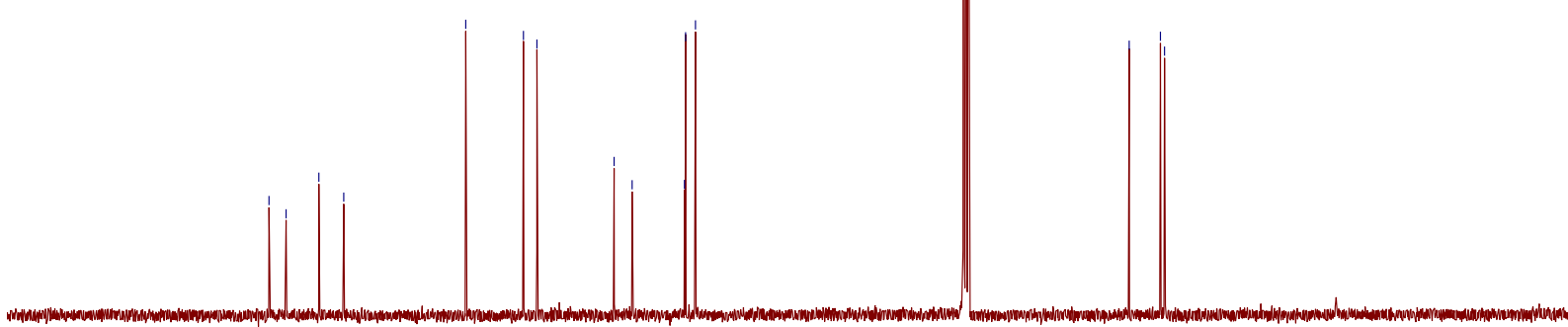

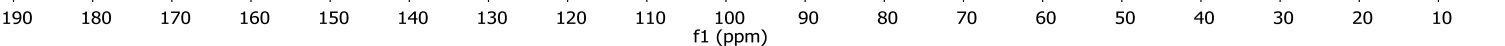


$41-{ }^{1} \mathrm{H}$ NMR $\left(400 \mathrm{MHz}, \mathrm{CDCl}_{3}\right)$

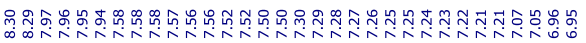<smiles>COC(=O)c1cc(-c2cc3ccccc3o2)ccc1OC</smiles>

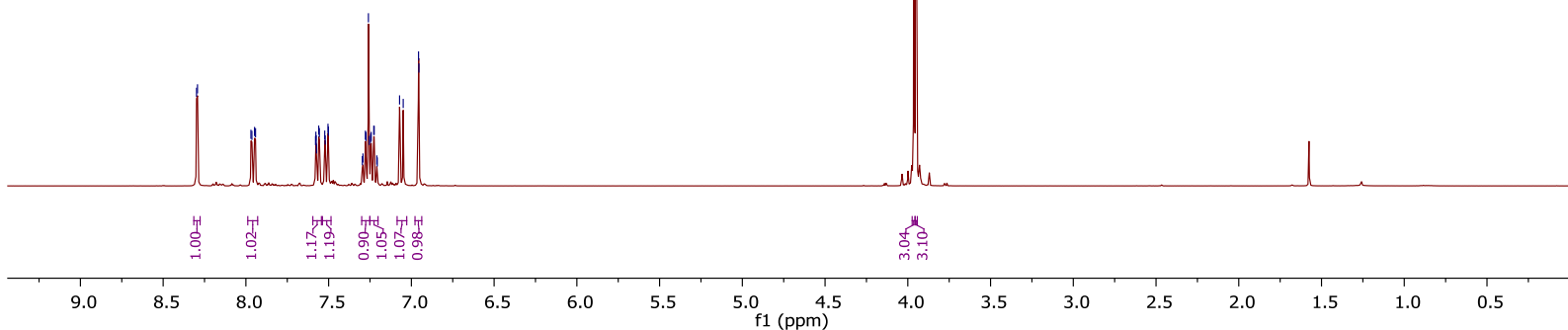

$41-{ }^{13} \mathrm{C} N M R\left(100 \mathrm{MHz}, \mathrm{CDCl}_{3}\right)$

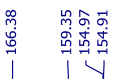<smiles>COC(=O)c1cc(-c2cc3ccccc3o2)ccc1OC</smiles> 
$42-{ }^{1} \mathrm{H}$ NMR $\left(400 \mathrm{MHz}, \mathrm{CDCl}_{3}\right)$

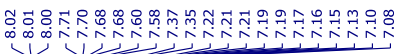

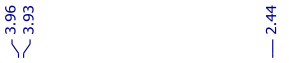<smiles>COc1ccc(-c2[nH]c3ccccc3c2N)cc1C(=O)O</smiles>

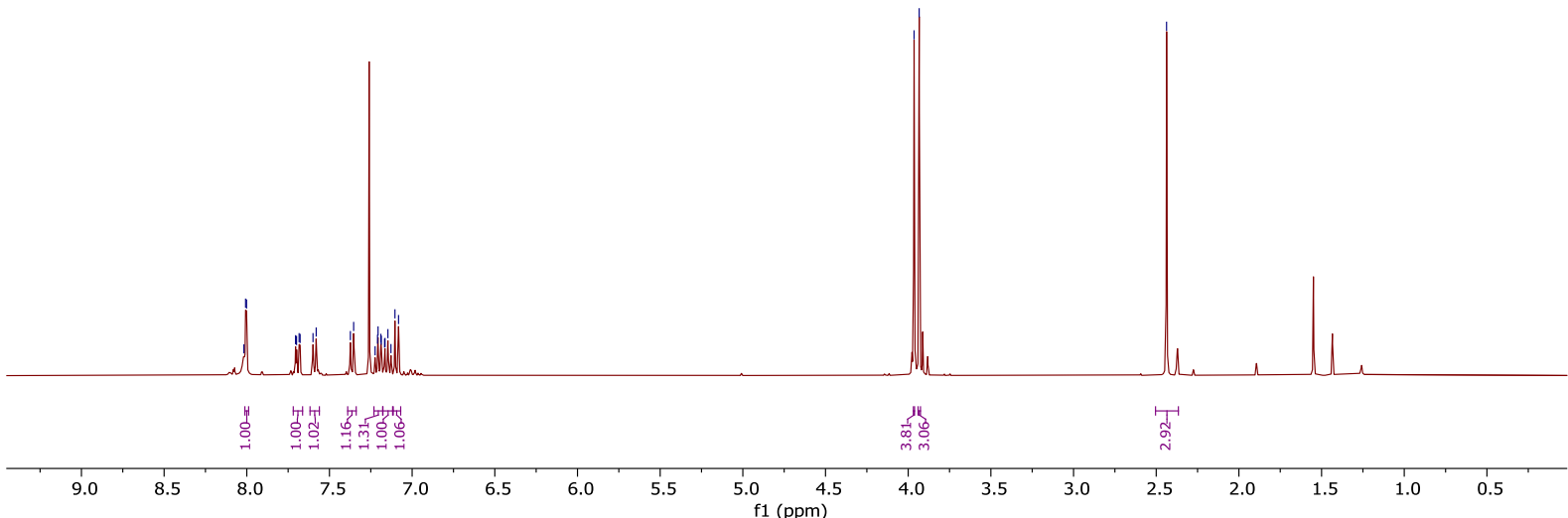

$42-{ }^{13} \mathrm{C}$ NMR (100 MHz, $\left.\mathrm{CDCl}_{3}\right)$

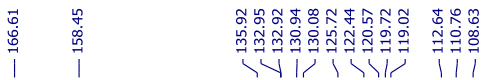

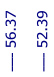<smiles>COC(=O)c1cc(-c2[nH]c3ccccc3c2C)ccc1OC</smiles> 
$43-{ }^{1} \mathrm{H}$ NMR $\left(400 \mathrm{MHz}, \mathrm{CDCl}_{3}\right)$

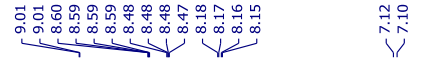<smiles>COc1ccc(-c2cnccn2)cc1C(=O)O</smiles>

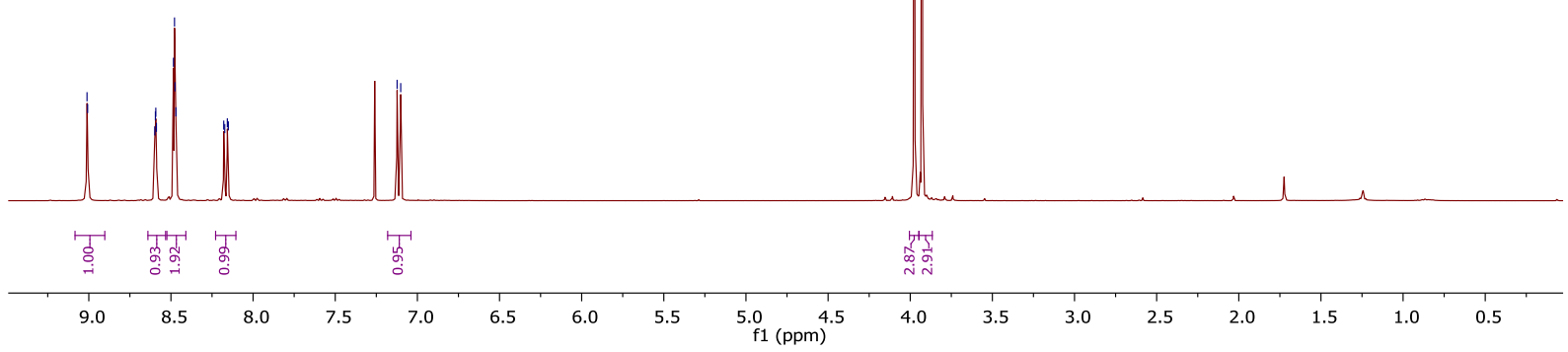

$43-{ }^{13} \mathrm{C}$ NMR (100 MHz, $\left.\mathrm{CDCl}_{3}\right)$

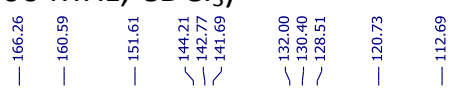<smiles>COC(=O)c1cc(-c2cnccn2)ccc1OC</smiles>

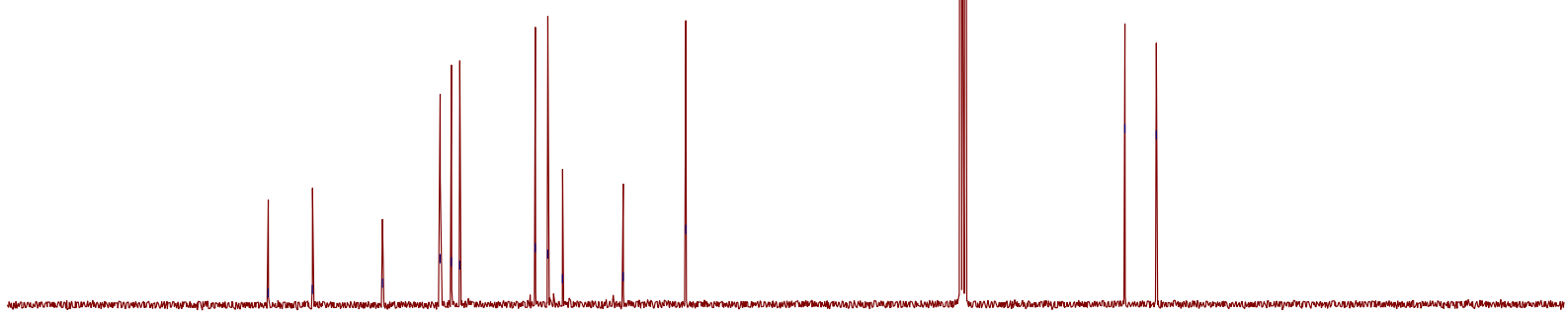

190

180

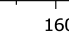

150

140

120 
$44-{ }^{1} \mathrm{H}$ NMR $\left(400 \mathrm{MHz}, \mathrm{CDCl}_{3}\right)$

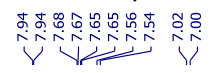

옹ํํำ

ป゚ำ
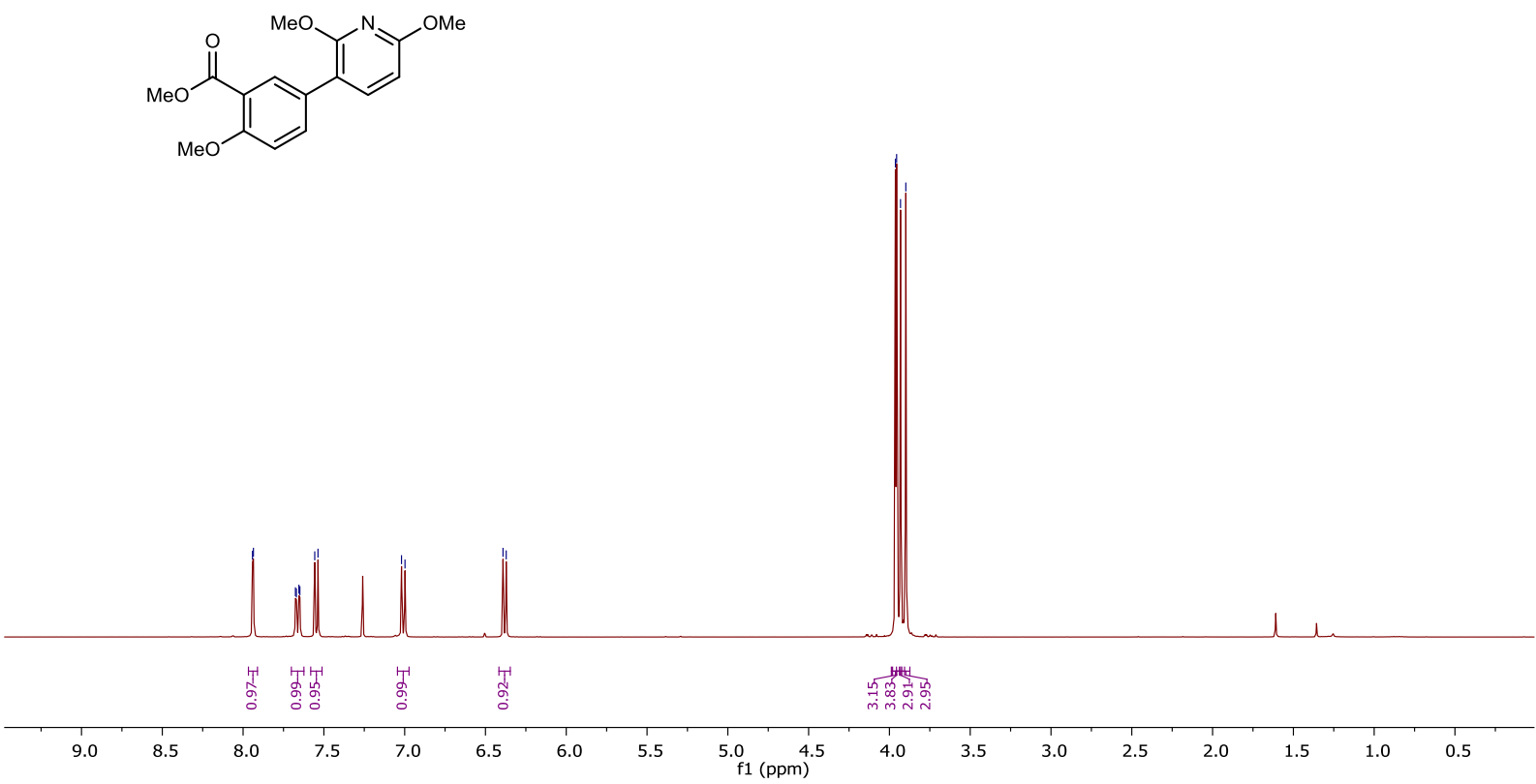

$44-{ }^{13} \mathrm{C}$ NMR $\left(100 \mathrm{MHz}, \mathrm{CDCl}_{3}\right)$

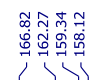

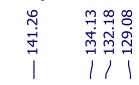

量琵

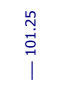

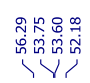
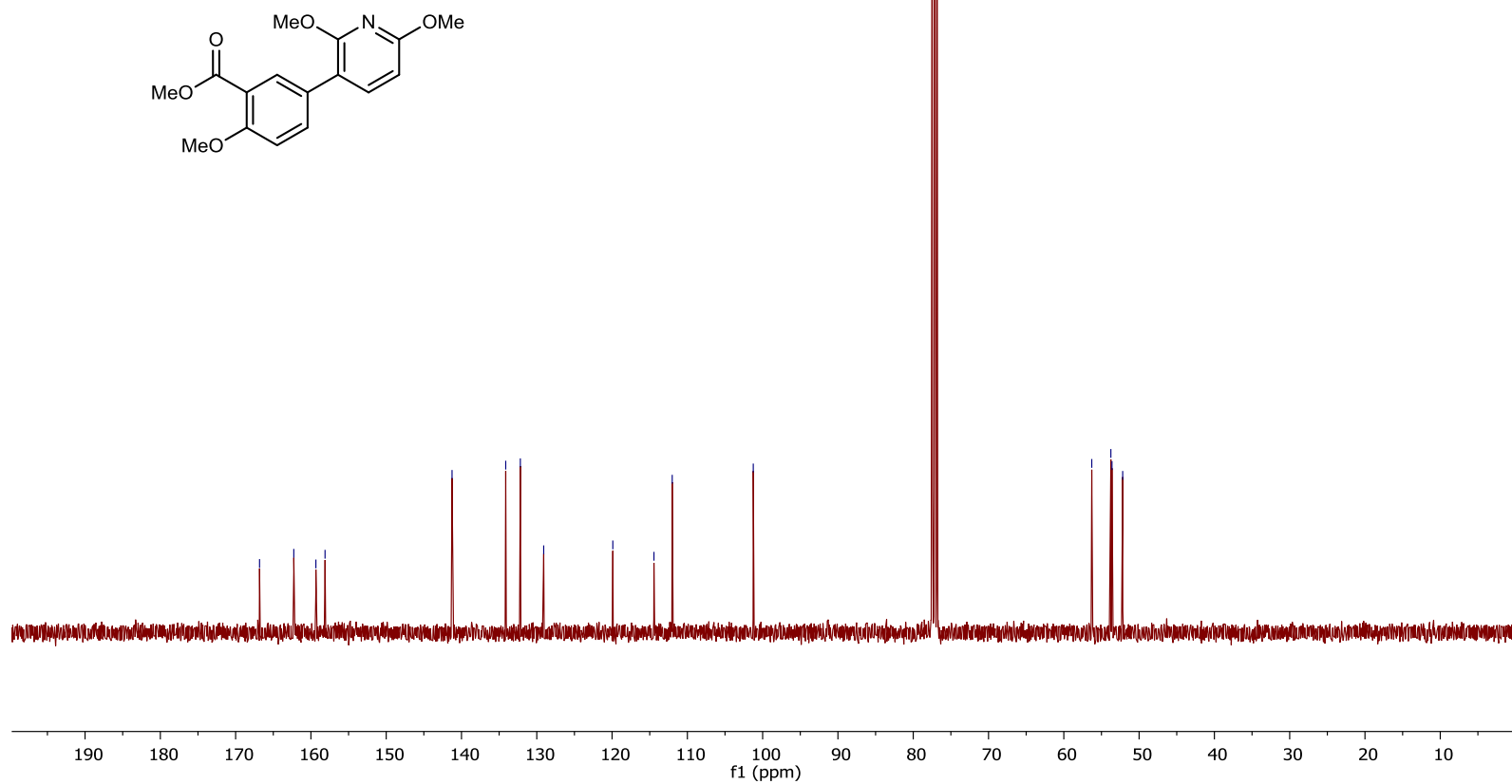

110 
$45-{ }^{1} \mathrm{H}$ NMR $\left(400 \mathrm{MHz}, \mathrm{CDCl}_{3}\right)$

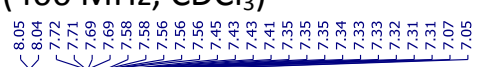

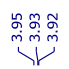
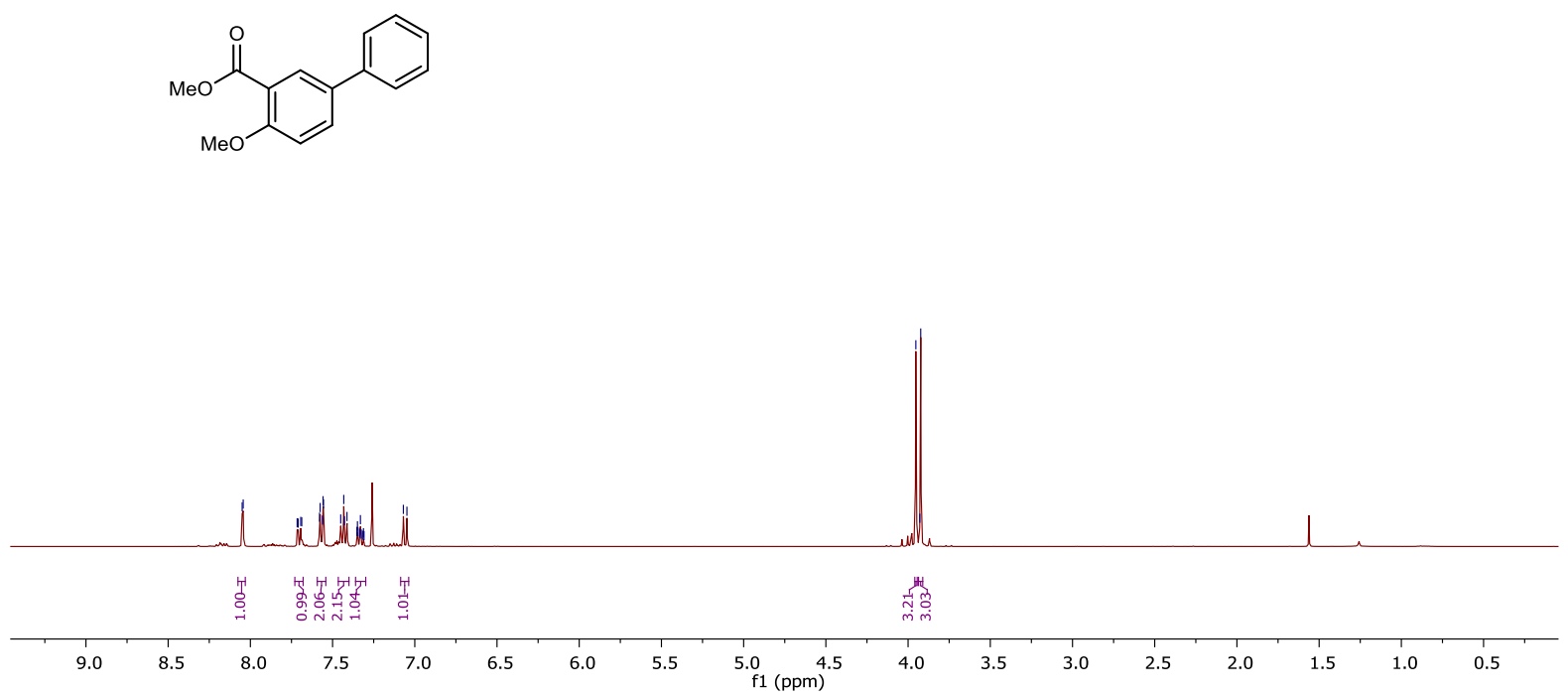

$45-{ }^{13} \mathrm{CNMR}\left(100 \mathrm{MHz}, \mathrm{CDCl}_{3}\right)$

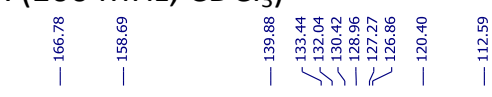

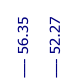
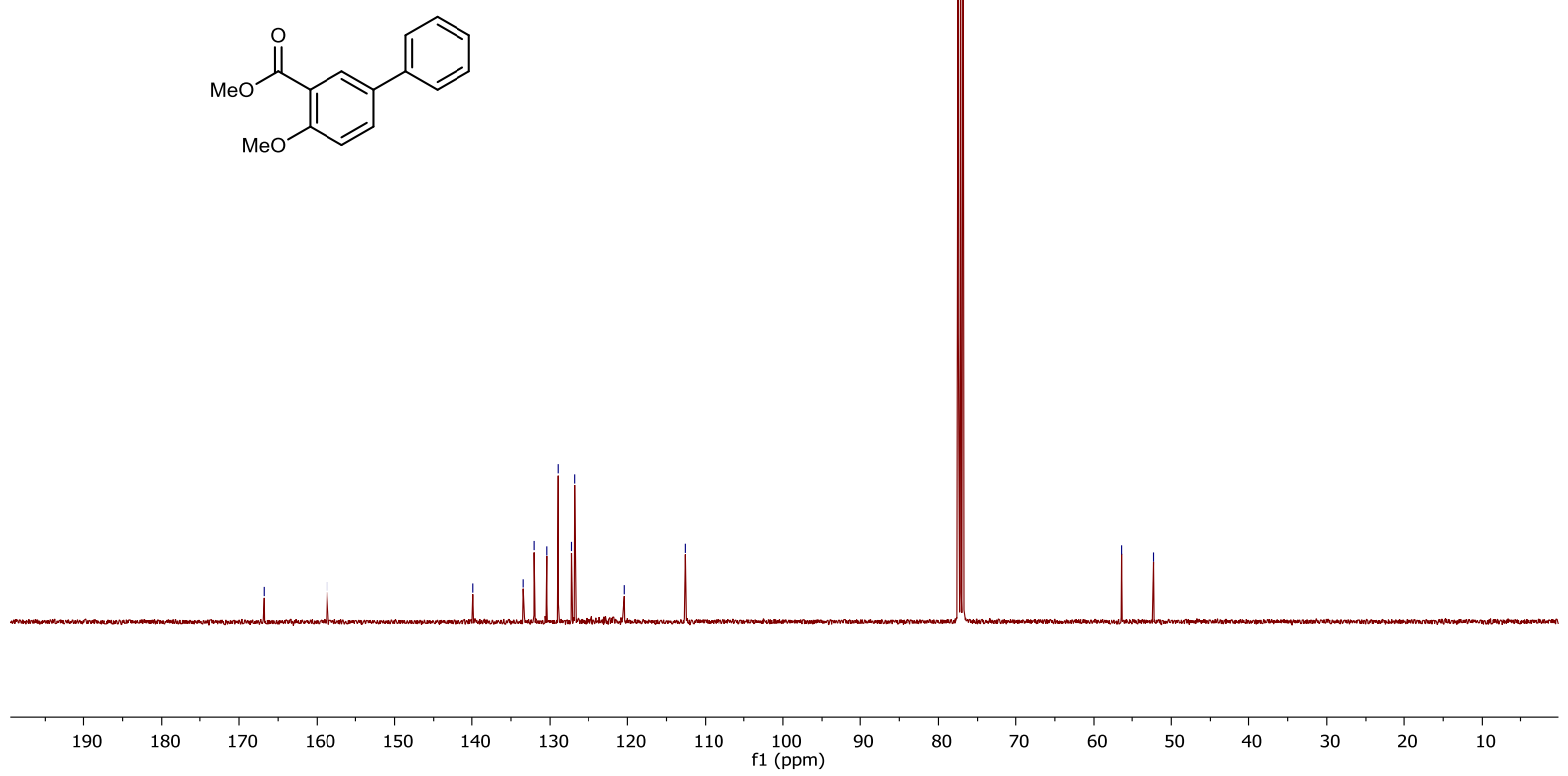

111 
$46-{ }^{1} \mathrm{H}$ NMR $\left(400 \mathrm{MHz}, \mathrm{CDCl}_{3}\right)$

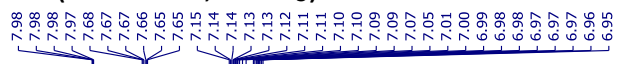

品高
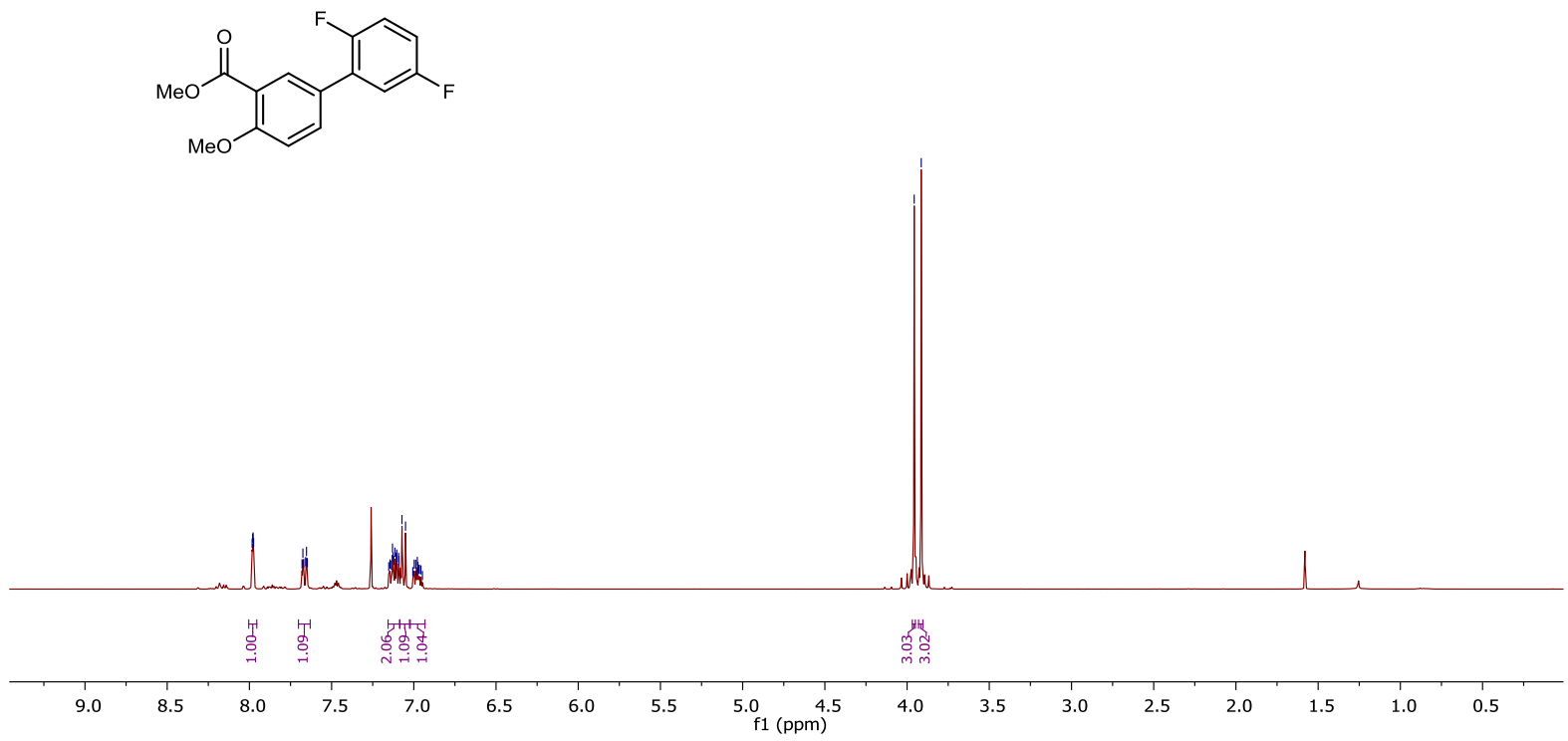

$46-{ }^{13} \mathrm{C}$ NMR $\left(100 \mathrm{MHz}, \mathrm{CDCl}_{3}\right)$

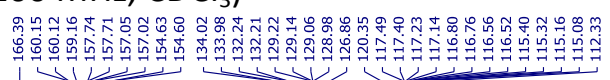

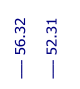
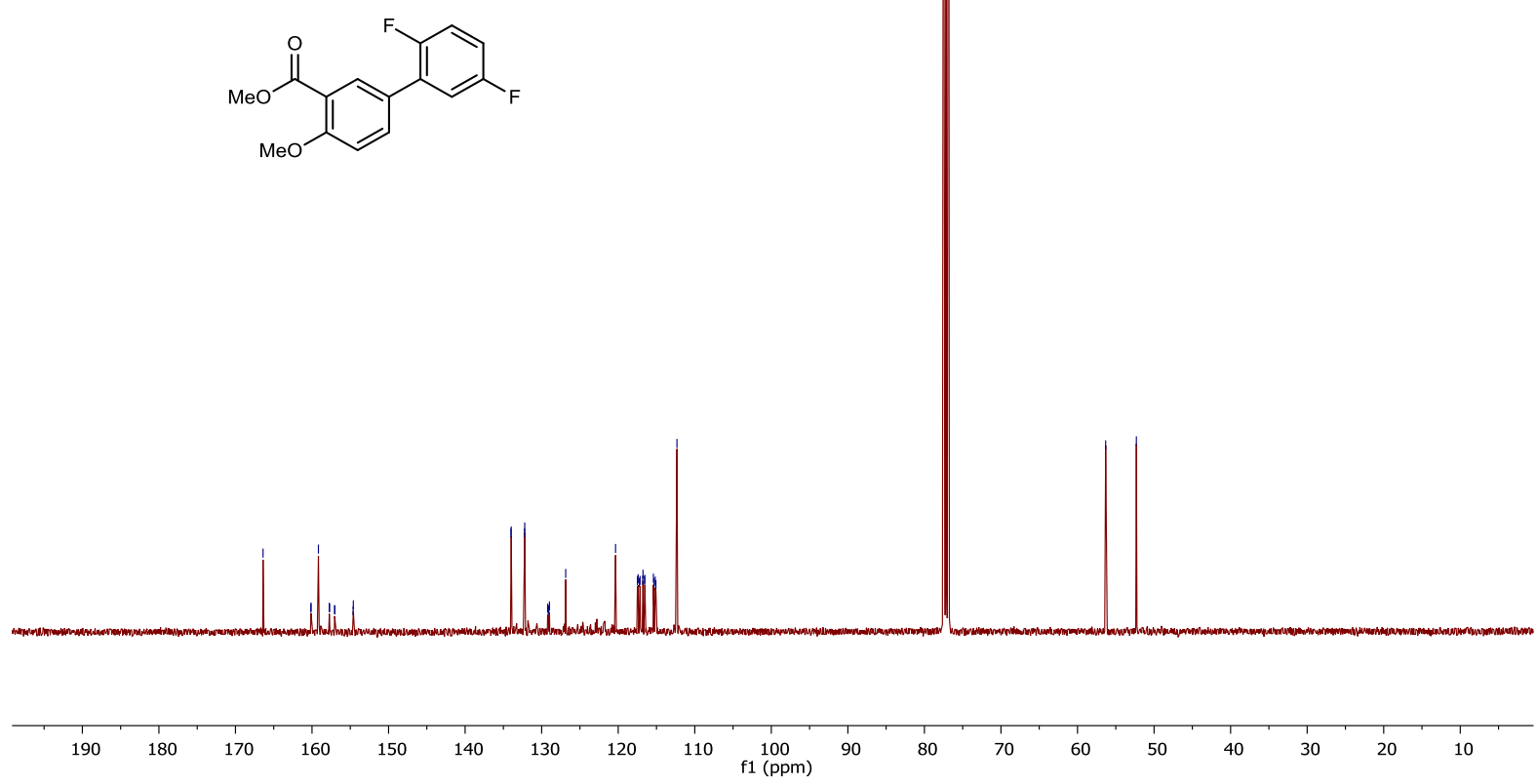

112 
$46-{ }^{19} \mathrm{~F}$ NMR (376 MHz, $\mathrm{CDCl}_{3}$ )

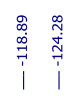
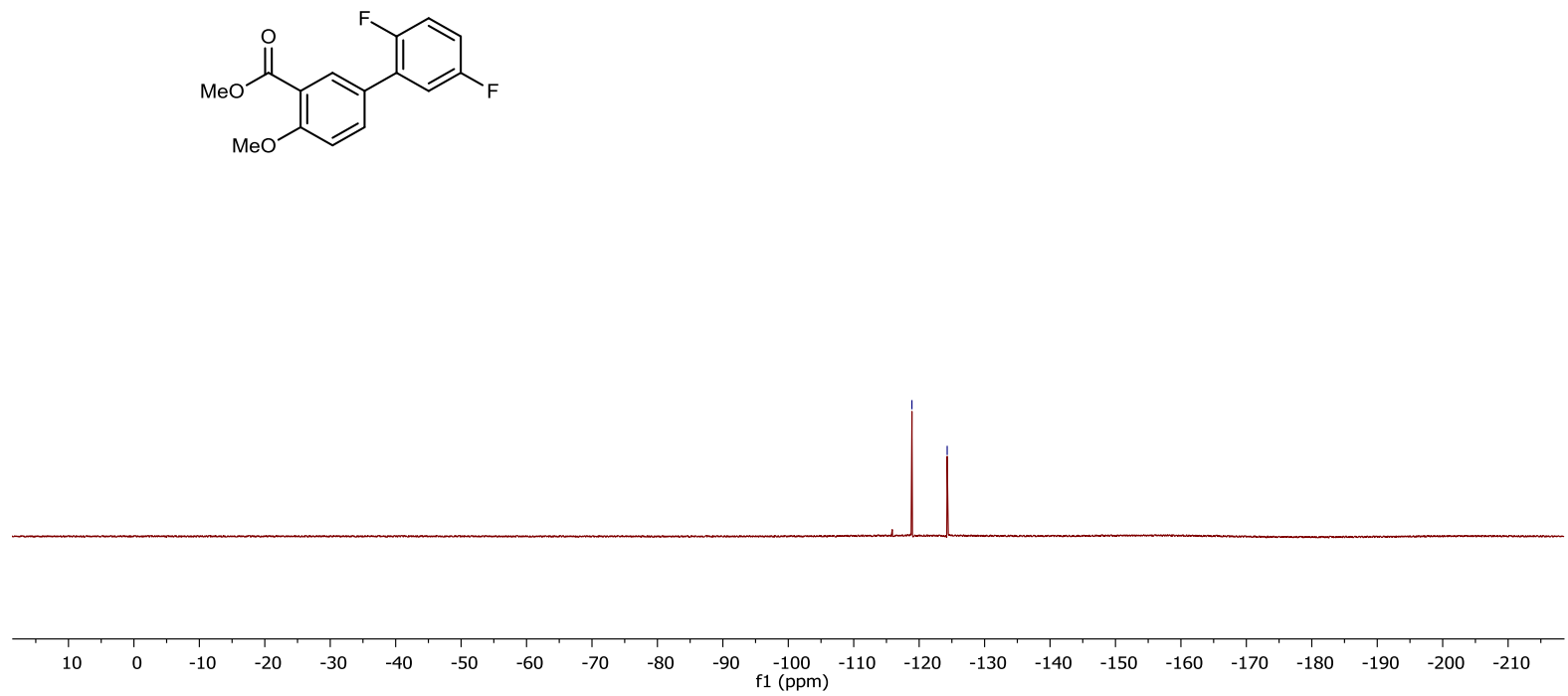

113 
$47-{ }^{1} \mathrm{H}$ NMR $\left(400 \mathrm{MHz}, \mathrm{CDCl}_{3}\right)$

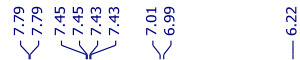<smiles>COC(=O)c1cc(-c2c(OC)cc(OC)cc2OC)ccc1OC</smiles>

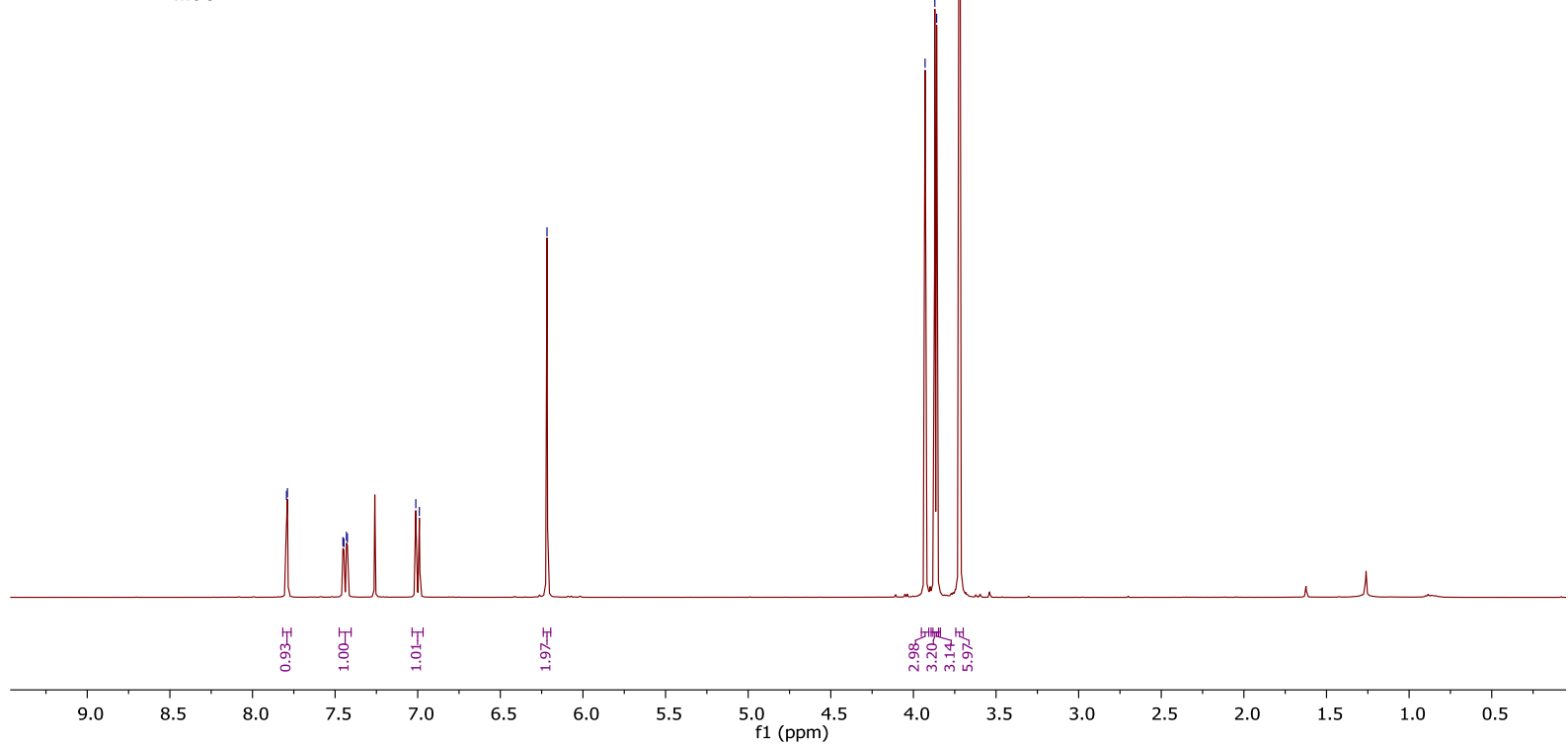

$47-{ }^{13} \mathrm{CNMR}\left(100 \mathrm{MHz}, \mathrm{CDCl}_{3}\right)$<smiles>COC(=O)c1cc(-c2c(OC)cc(OC)cc2OC)ccc1OC</smiles>

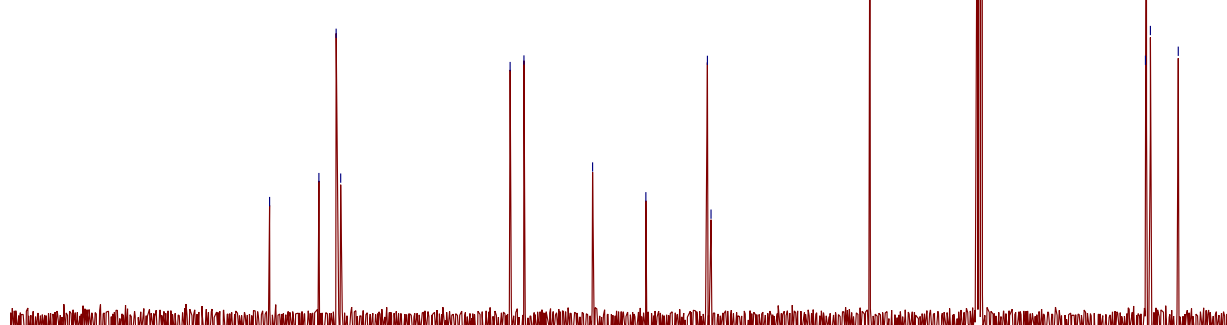


$48-{ }^{1} \mathrm{H}$ NMR (400 MHz, $\mathrm{CDCl}_{3}$ )

管

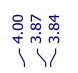
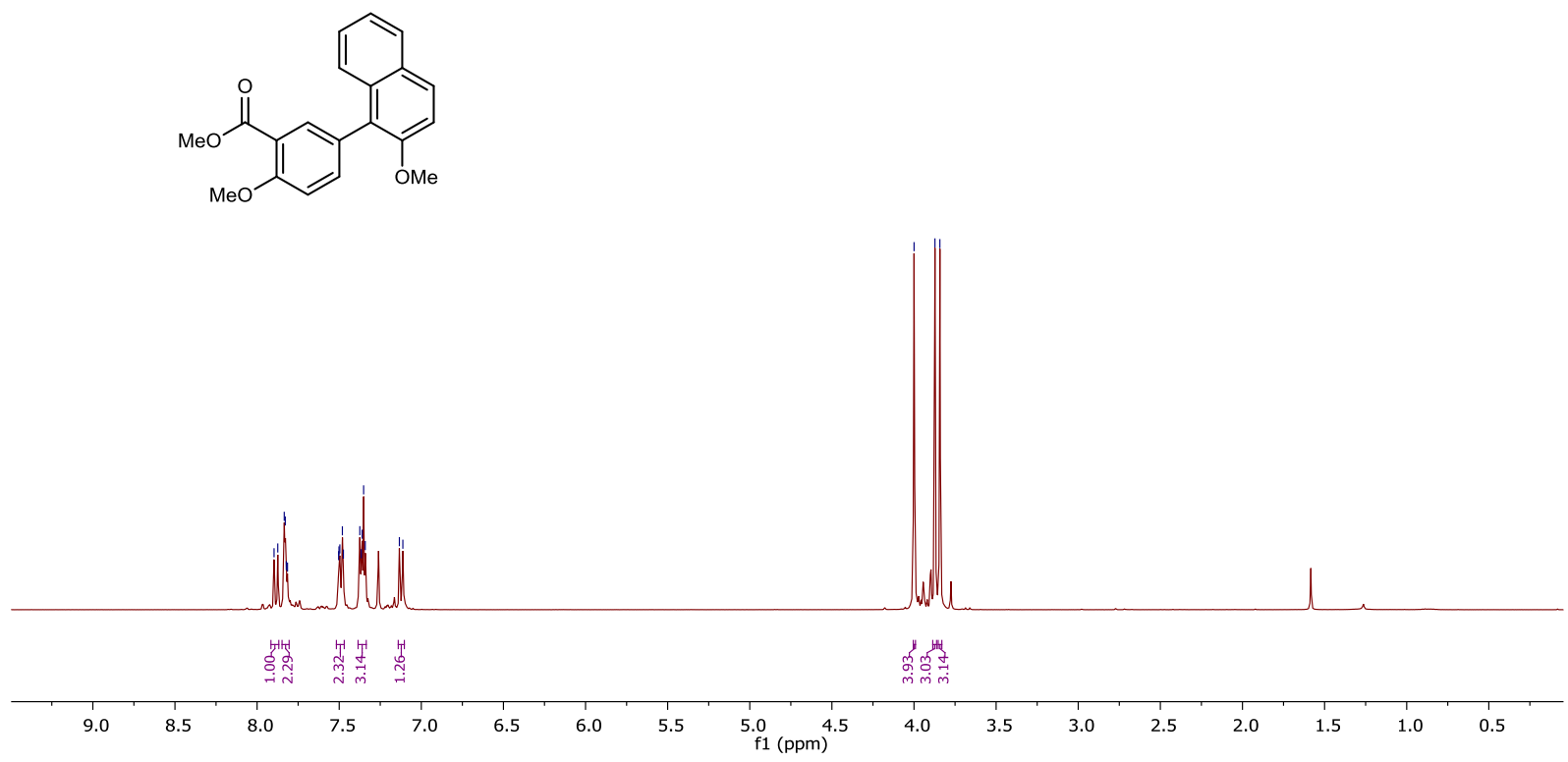

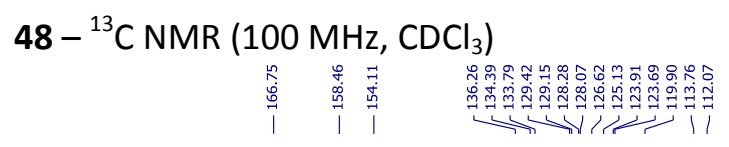

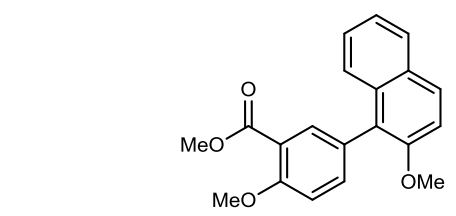

|r|

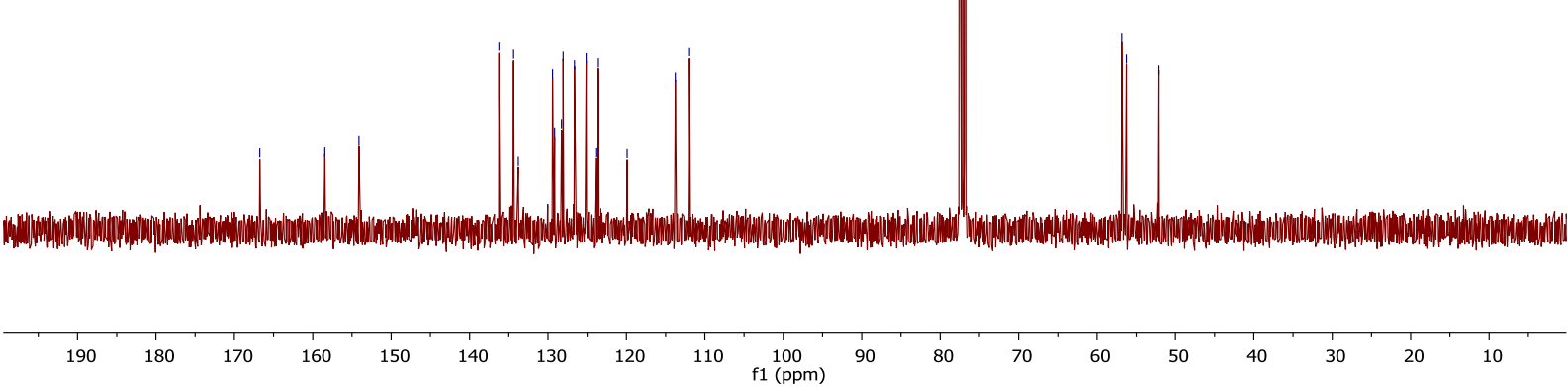

115 
S11 - ${ }^{1} \mathrm{H}$ NMR $\left(400 \mathrm{MHz}, \mathrm{CDCl}_{3}\right)$

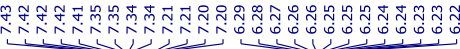

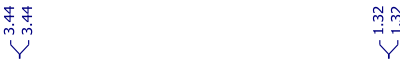<smiles>COC(=O)n1cccc1-c1cc(Cl)cc(Cl)c1OC</smiles>

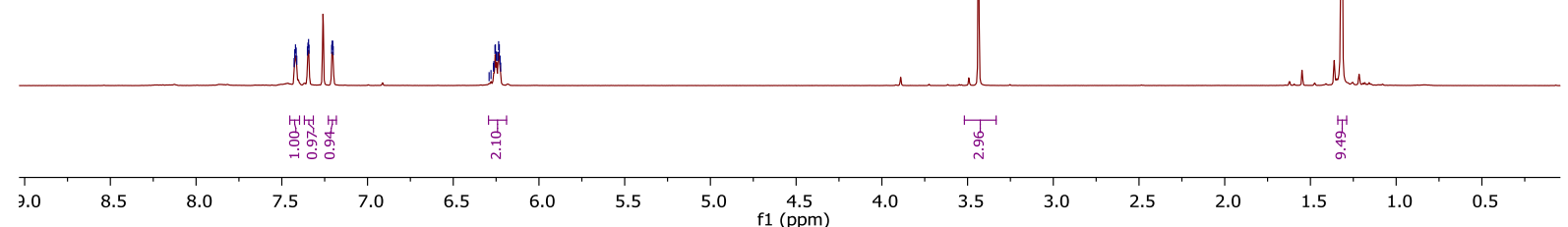

S11 $-{ }^{13} \mathrm{C}$ NMR $\left(100 \mathrm{MHz}, \mathrm{CDCl}_{3}\right)$

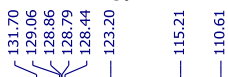<smiles>CCCCOC(=O)n1cccc1-c1cc(Cl)cc(Cl)c1OC</smiles>
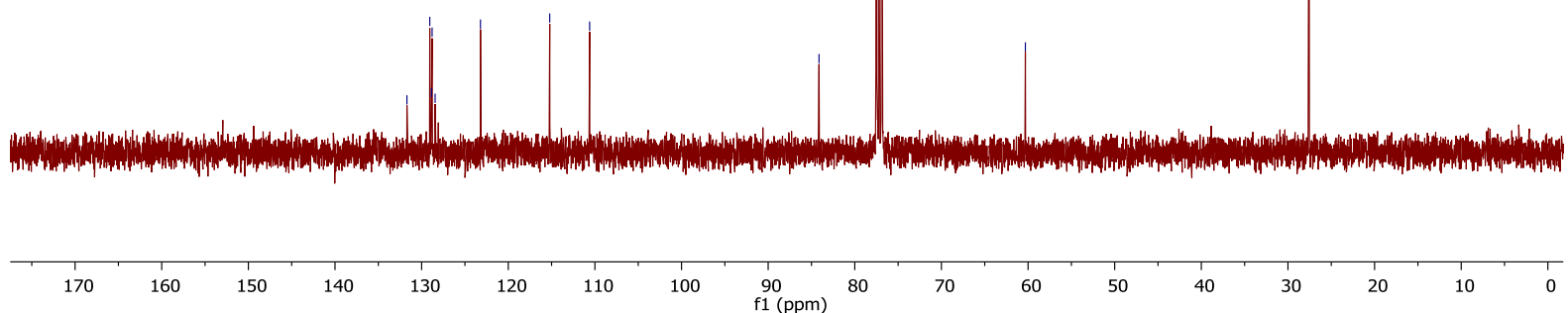
$49-{ }^{1} \mathrm{H}$ NMR $\left(500 \mathrm{MHz}, \mathrm{CDCl}_{3}\right)$

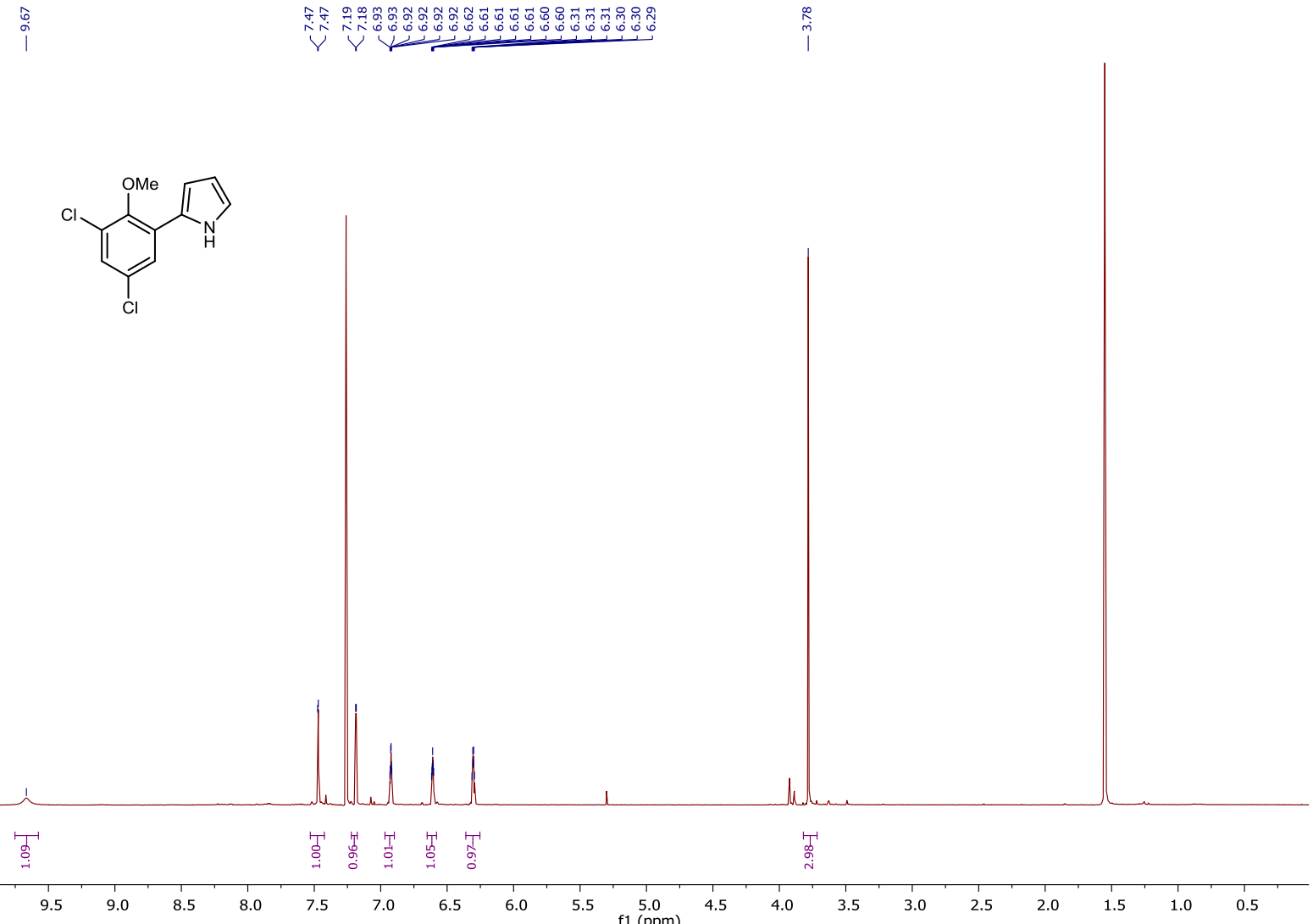

$49-{ }^{13} \mathrm{C}$ NMR $\left(125 \mathrm{MHz}, \mathrm{CDCl}_{3}\right)$<smiles>COc1c(Cl)cc(Cl)cc1-c1ccc[nH]1</smiles>

indw. 
$50-{ }^{1} \mathrm{H}$ NMR (400 MHz, $\left.\mathrm{CDCl}_{3}\right)$

พั

ํํำ

i
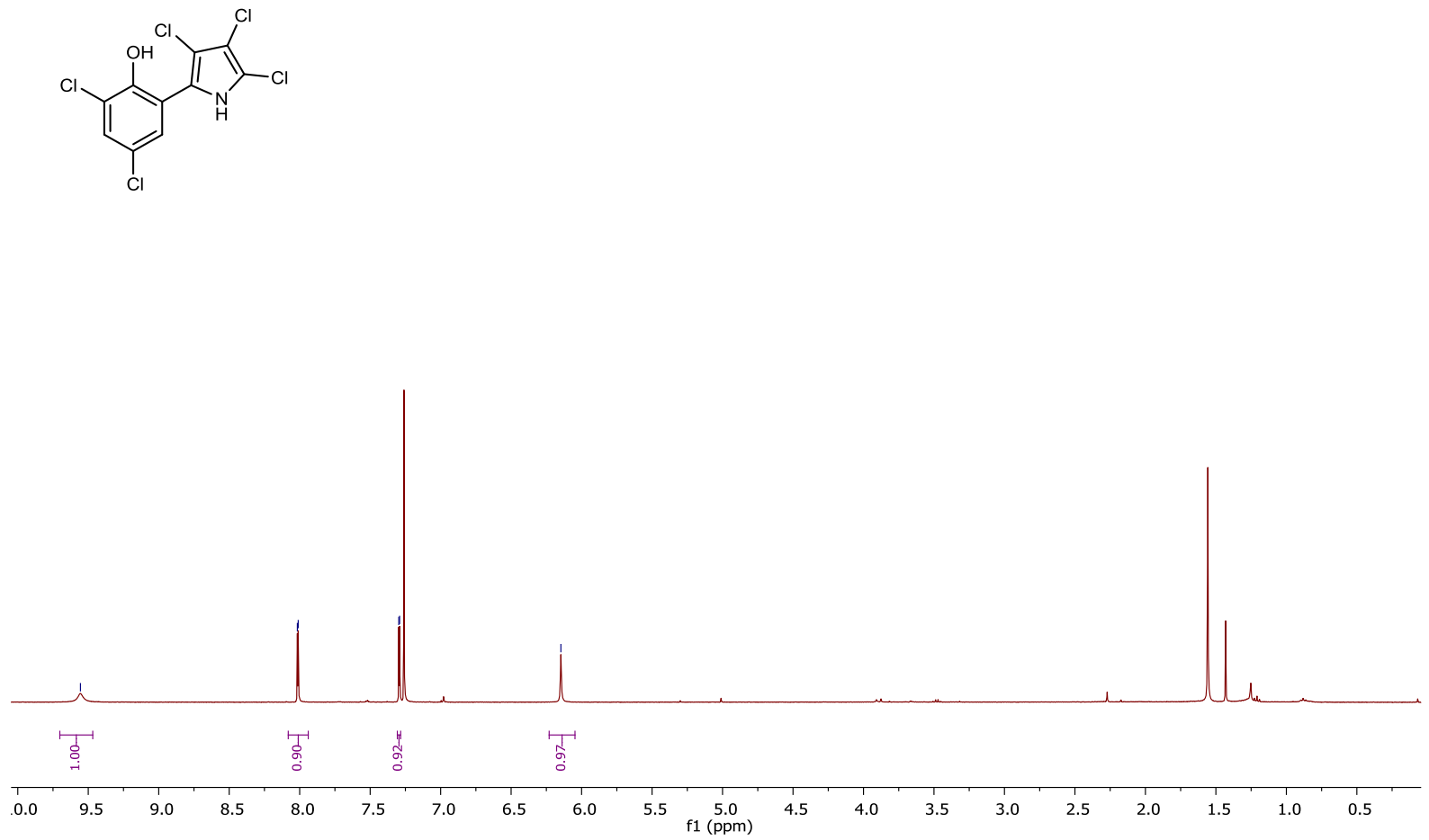

$50-{ }^{13} \mathrm{CNMR}\left(100 \mathrm{MHz}, \mathrm{CDCl}_{3}\right)$
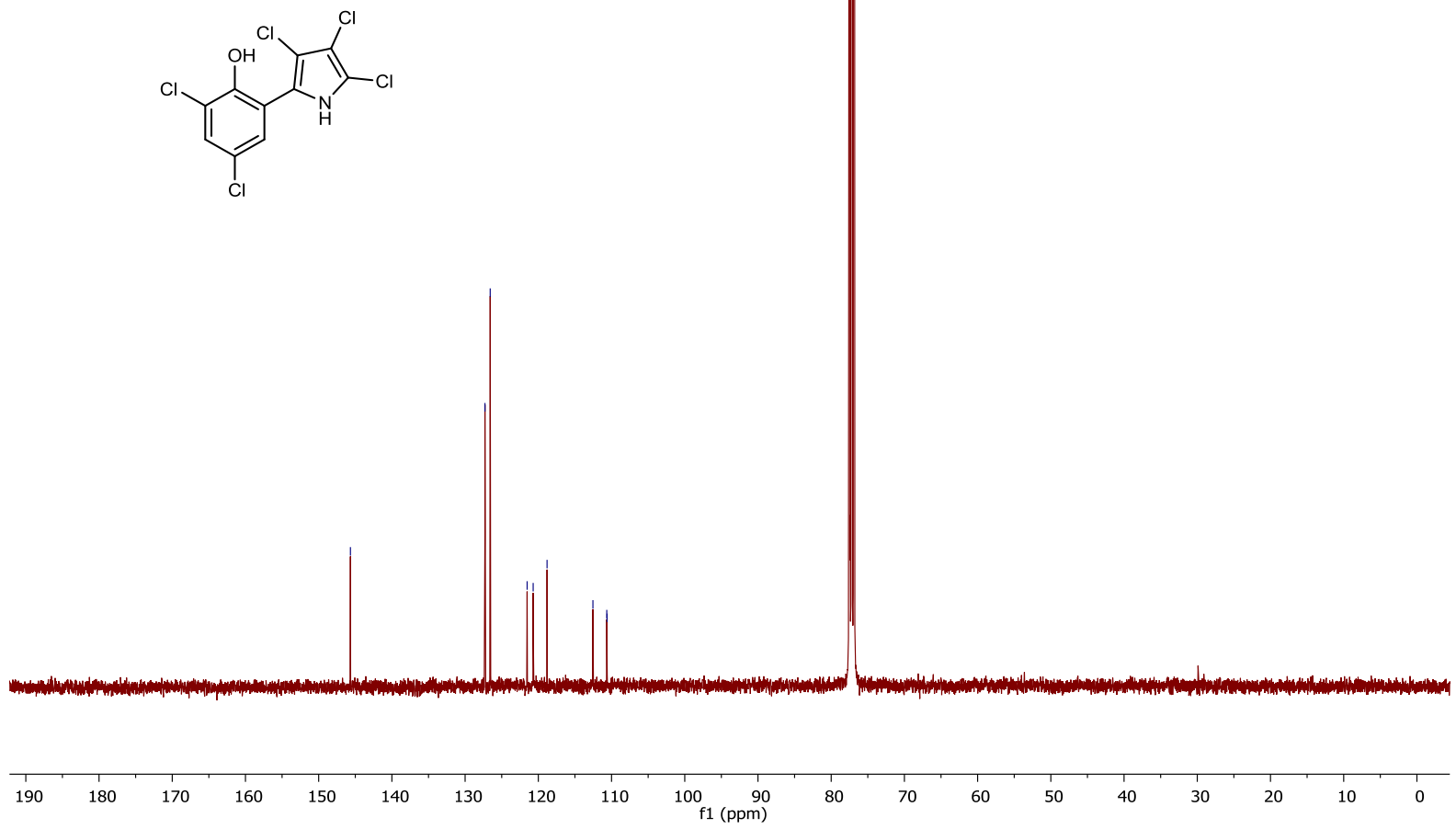

118 
S12 $-{ }^{1} \mathrm{H}$ NMR $\left(400 \mathrm{MHz}, \mathrm{CDCl}_{3}\right)$

每<smiles>Oc1cc(Cl)cc2c(Br)c(Br)[nH]c12</smiles>

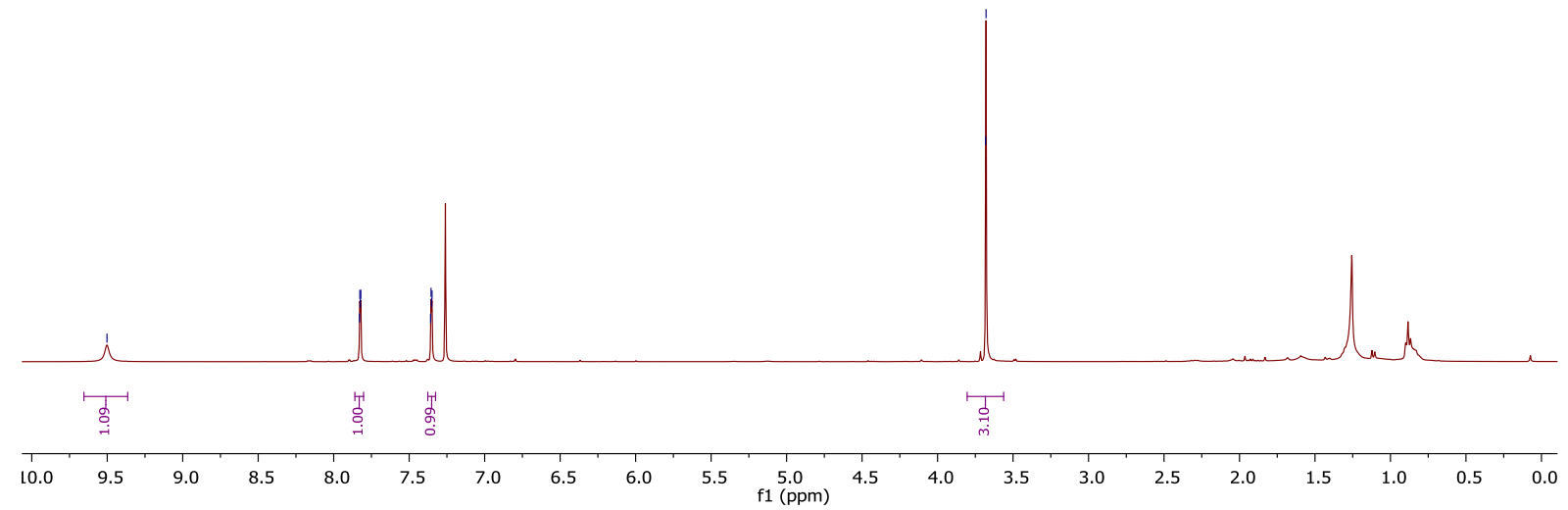

S12 $-{ }^{13} \mathrm{C}$ NMR $\left(100 \mathrm{MHz}, \mathrm{CDCl}_{3}\right)$
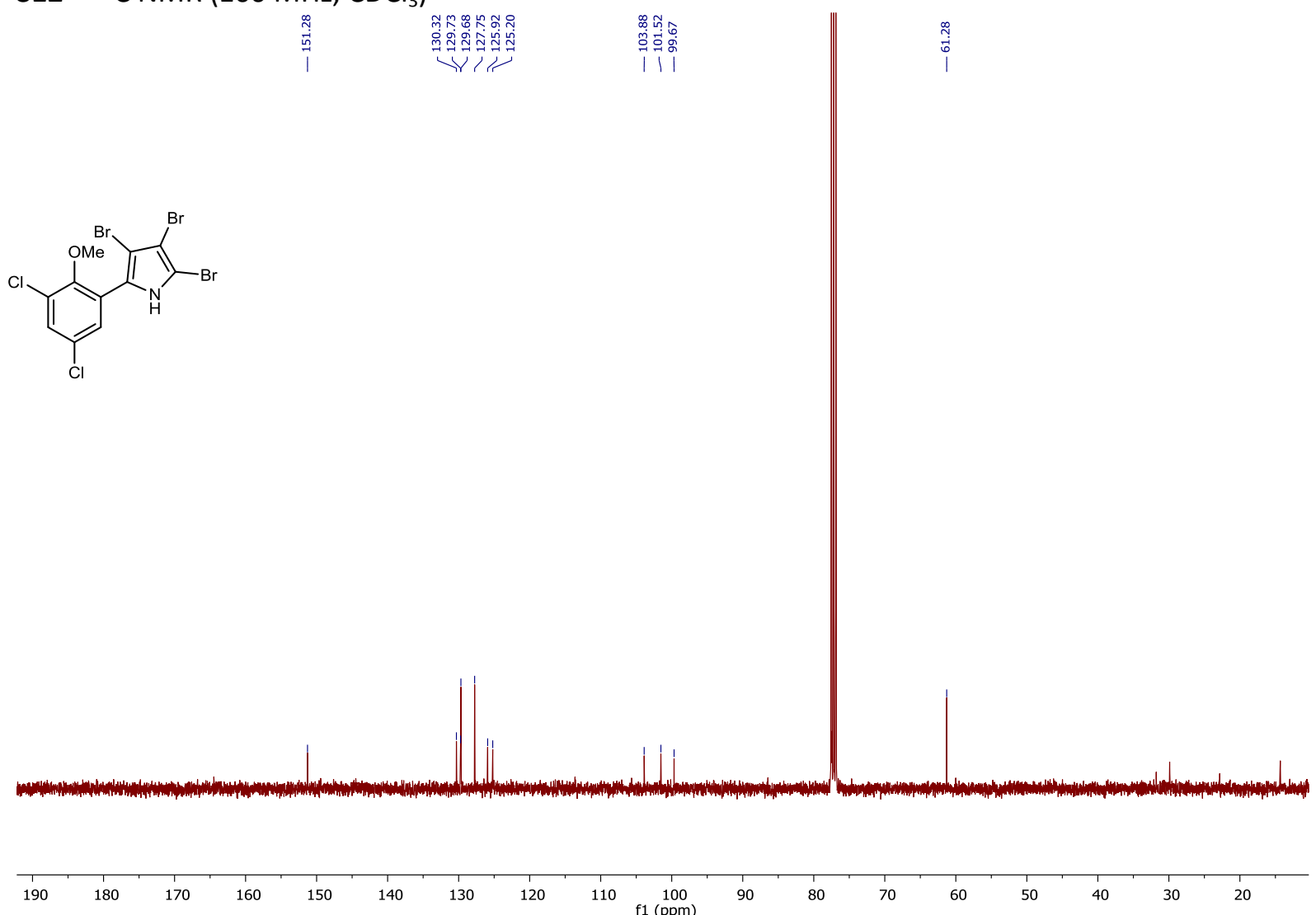
$51-{ }^{1} \mathrm{H}$ NMR $\left(400 \mathrm{MHz}, \mathrm{CDCl}_{3}\right)$

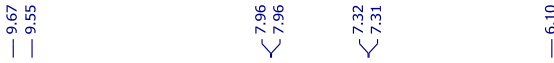<smiles>Oc1cc(Cl)cc(-c2[nH]c(Br)c(Br)c2Br)c1Br</smiles>

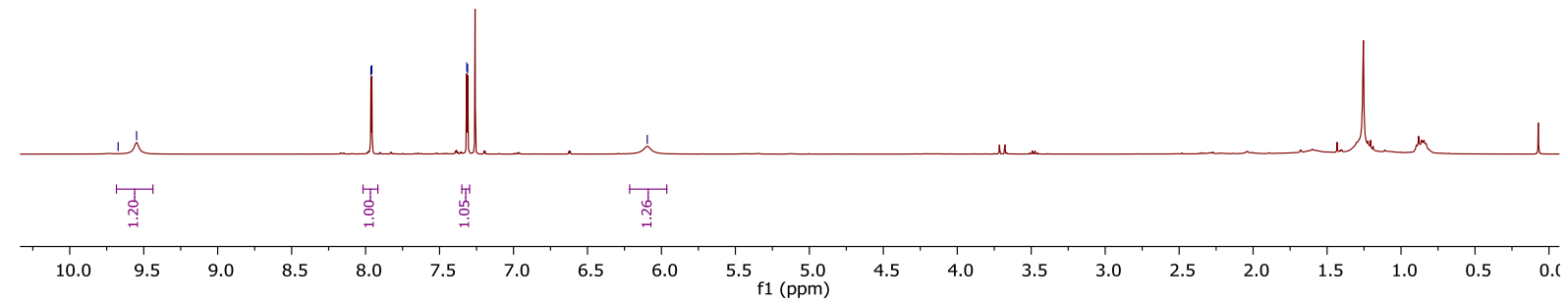

$51-{ }^{13} \mathrm{C}$ NMR (100 MHz, $\mathrm{CDCl}_{3}$ )
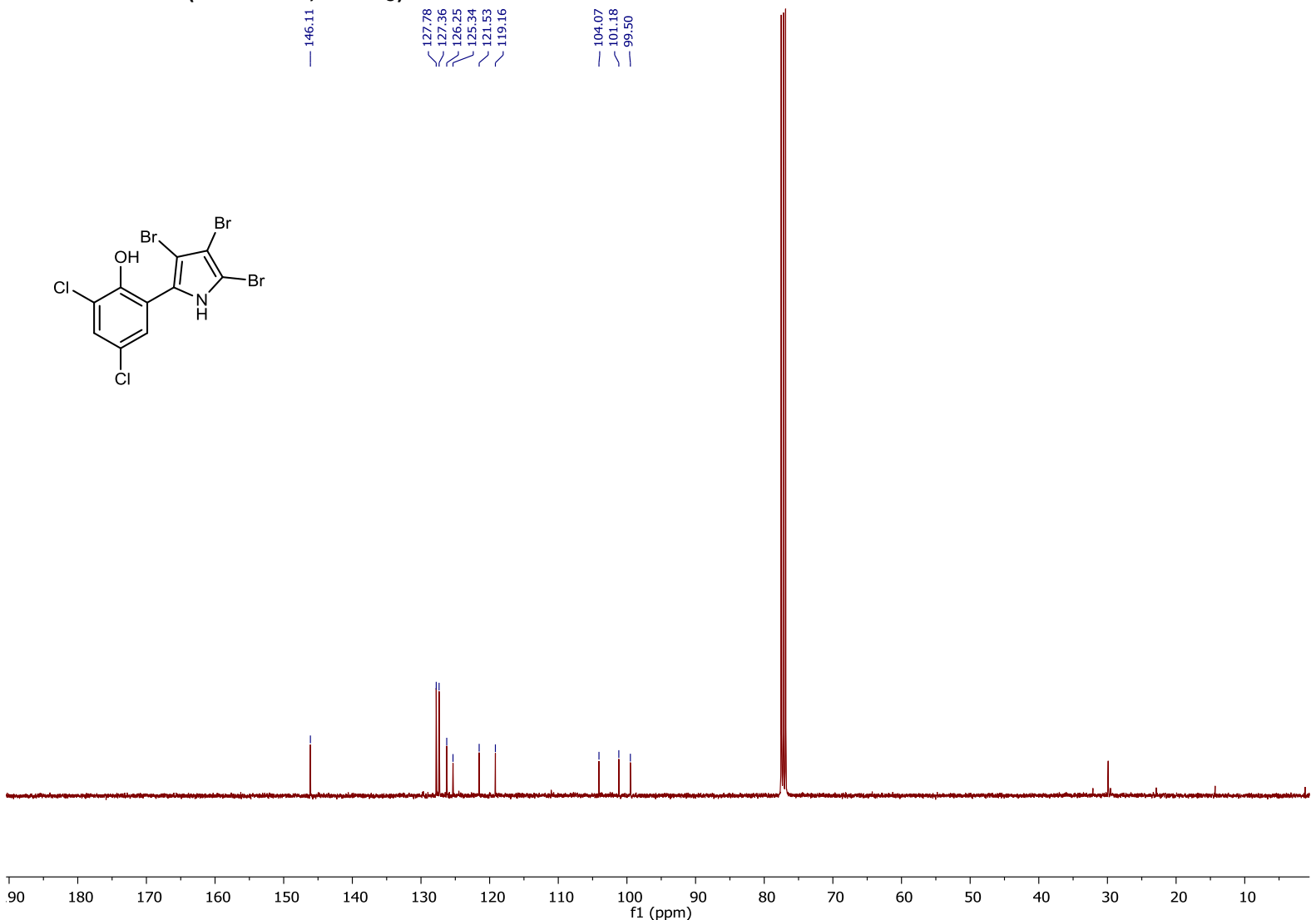

f1 (ppm)

80

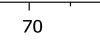

60

$20 \quad 10$ 
$52-{ }^{1} \mathrm{H}$ NMR $\left(400 \mathrm{MHz}, \mathrm{CDCl}_{3}\right)$

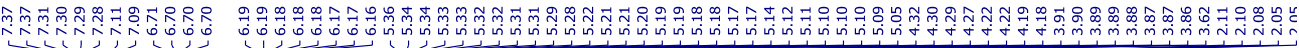

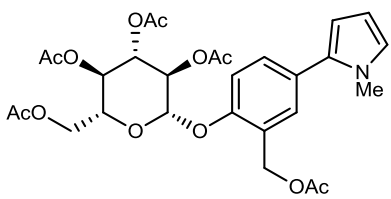

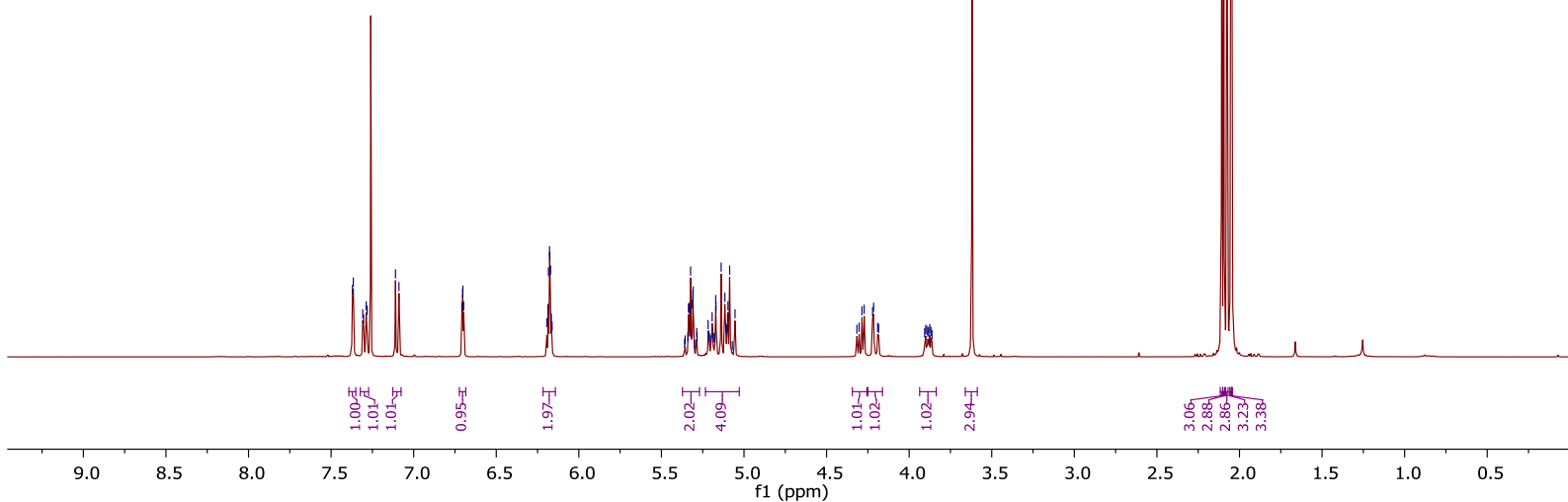

$52-{ }^{13} \mathrm{C}$ NMR $\left(100 \mathrm{MHz}, \mathrm{CDCl}_{3}\right)$

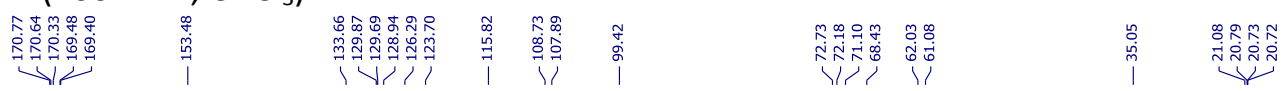<smiles>CC(=O)OCc1cc(-c2cccn2C)ccc1O[C@H]1O[C@H](COC(C)=O)[C@@H](OC(C)=O)[C@H]1OC(C)=O</smiles>

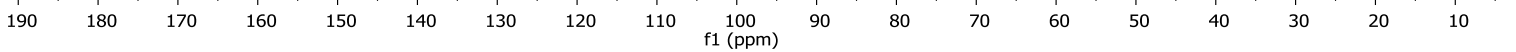


$53-{ }^{1} \mathrm{H}$ NMR $\left(400 \mathrm{MHz}, \mathrm{CDCl}_{3}\right)$

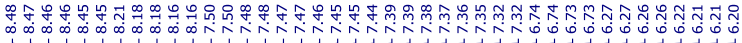<smiles>Cn1cccc1-c1ccc(NC(=O)c2cccnc2Cl)c(-c2ccc(Cl)cc2)c1</smiles>

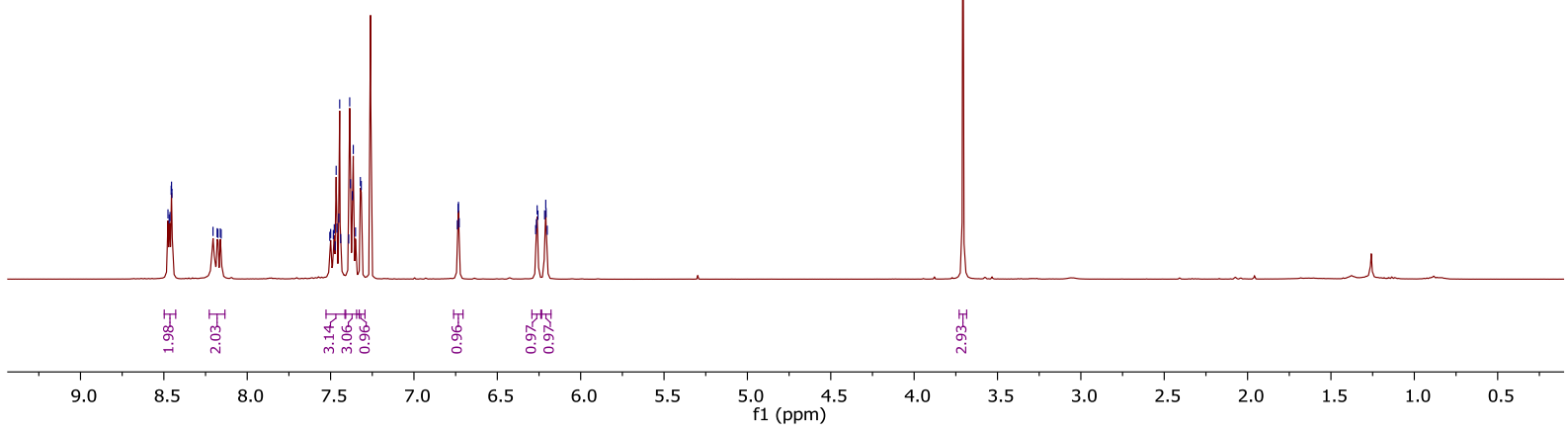

$53-{ }^{13} \mathrm{C}$ NMR $\left(100 \mathrm{MHz}, \mathrm{CDCl}_{3}\right)$

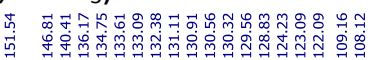

1 1<smiles>Cn1cccc1-c1ccc(NC(=O)c2cccnc2Cl)c(-c2ccc(Cl)cc2)c1</smiles>

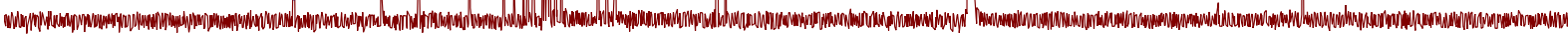

$\begin{array}{lll}190 & 180 & 170\end{array}$

150

140

120

$\stackrel{100}{\mathrm{f} 1(\mathrm{ppm})}$

$80 \quad 70$

$60 \quad 50$

40 
$54-{ }^{1} \mathrm{H}$ NMR $\left(400 \mathrm{MHz}, \mathrm{CDCl}_{3}\right)$

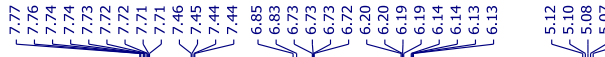
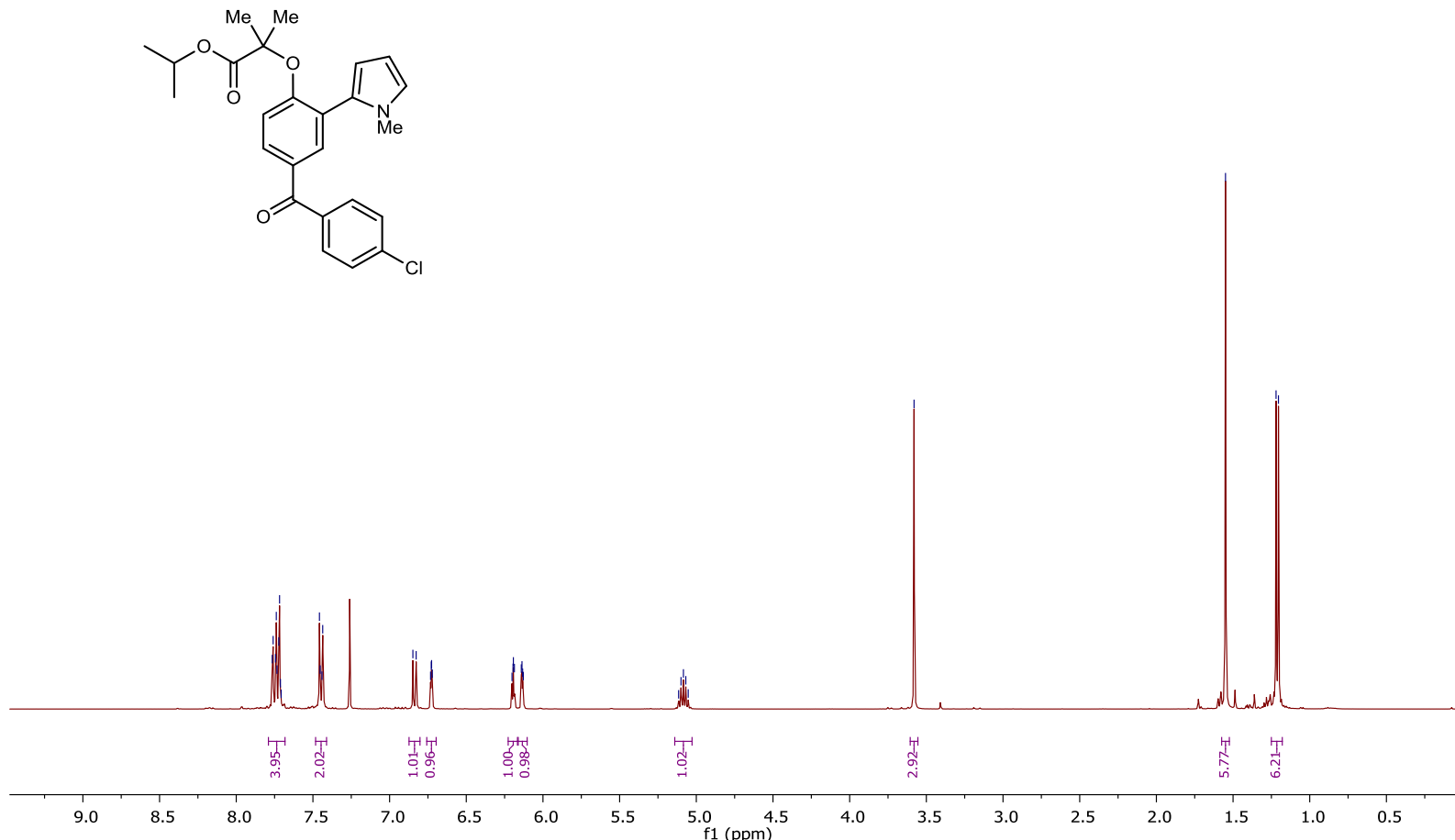

$54-{ }^{13} \mathrm{C}$ NMR (100 MHz, $\left.\mathrm{CDCl}_{3}\right)$

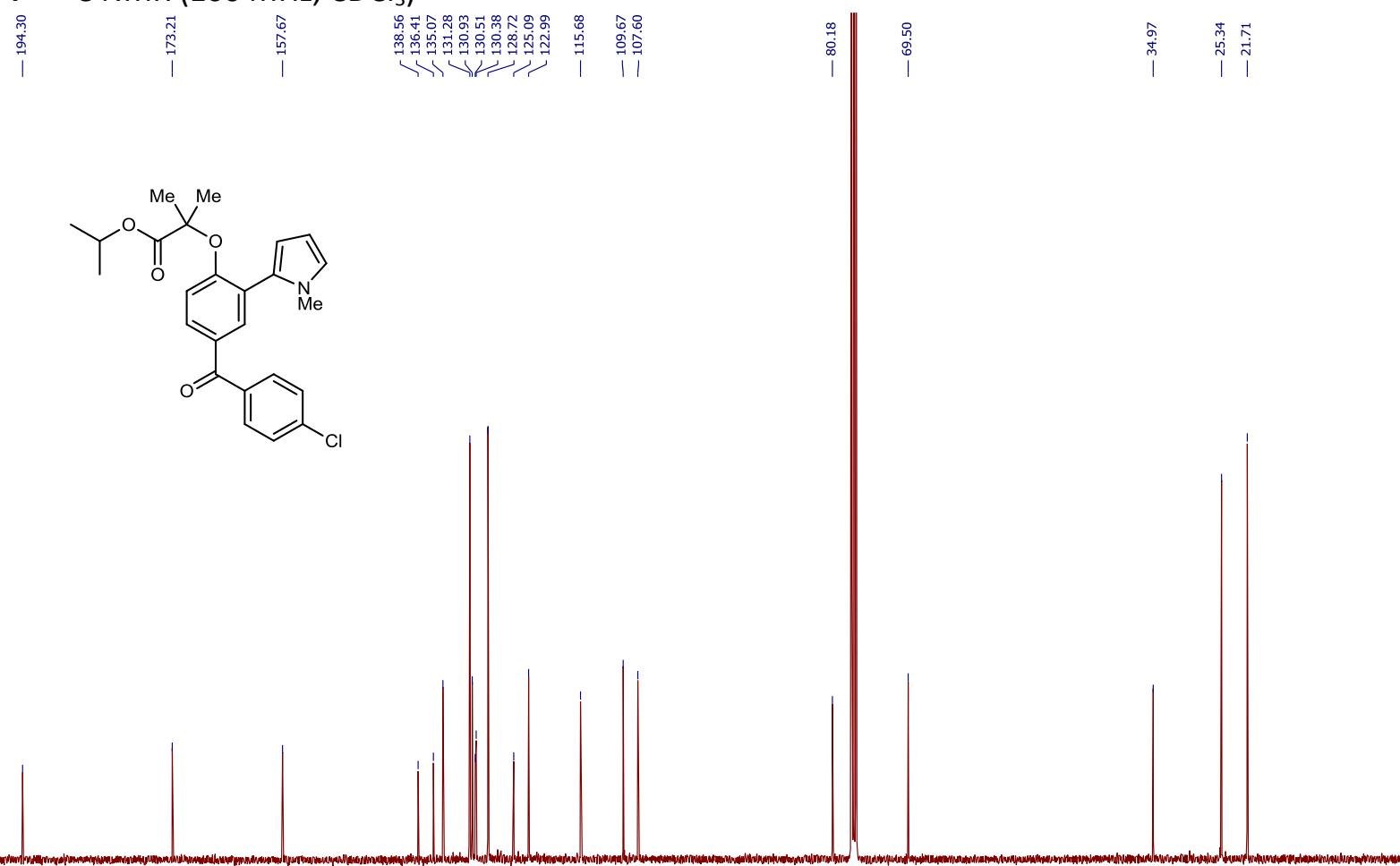

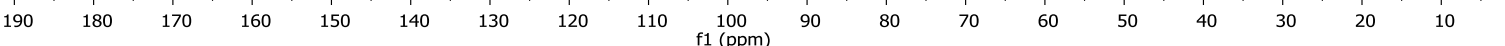


$55-{ }^{1} \mathrm{H}$ NMR $\left(400 \mathrm{MHz}, \mathrm{CDCl}_{3}\right)$

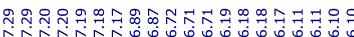<smiles></smiles>

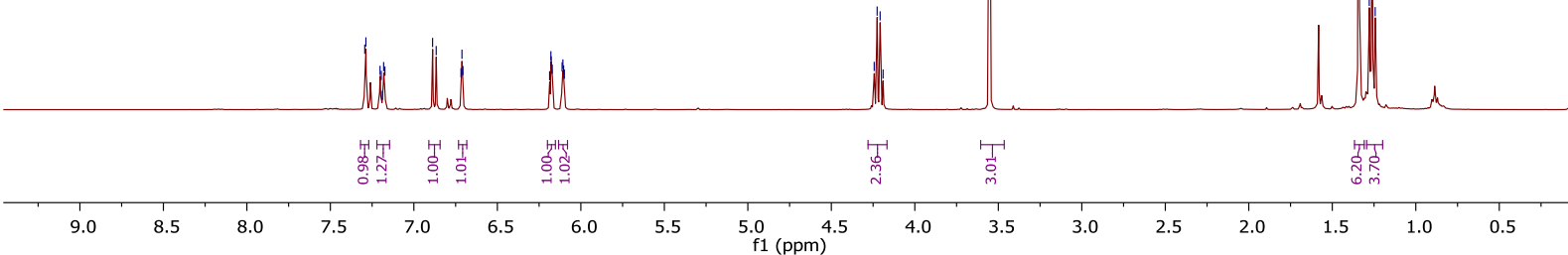

$55-{ }^{13} \mathrm{C}$ NMR $\left(100 \mathrm{MHz}, \mathrm{CDCl}_{3}\right)$

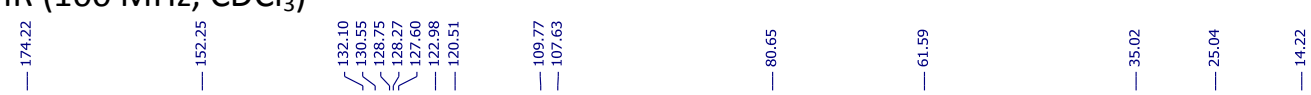<smiles></smiles>

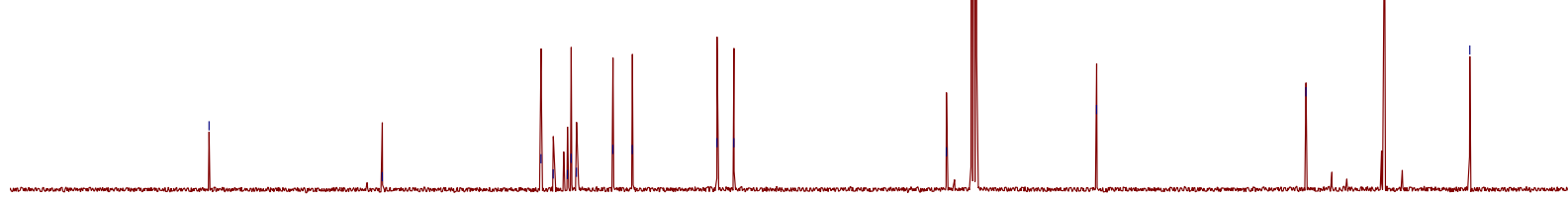

$\begin{array}{llllllllll}190 & 180 & 170 & 160 & 150 & 140 & 130 & 120 & 110 & \begin{array}{c}100 \\ \mathrm{f} 1(\mathrm{ppm})\end{array}\end{array}$ 
$56-{ }^{1} \mathrm{H}$ NMR $\left(400 \mathrm{MHz}, \mathrm{CDCl}_{3}\right)$

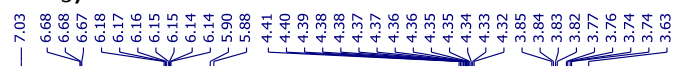

₹ิ
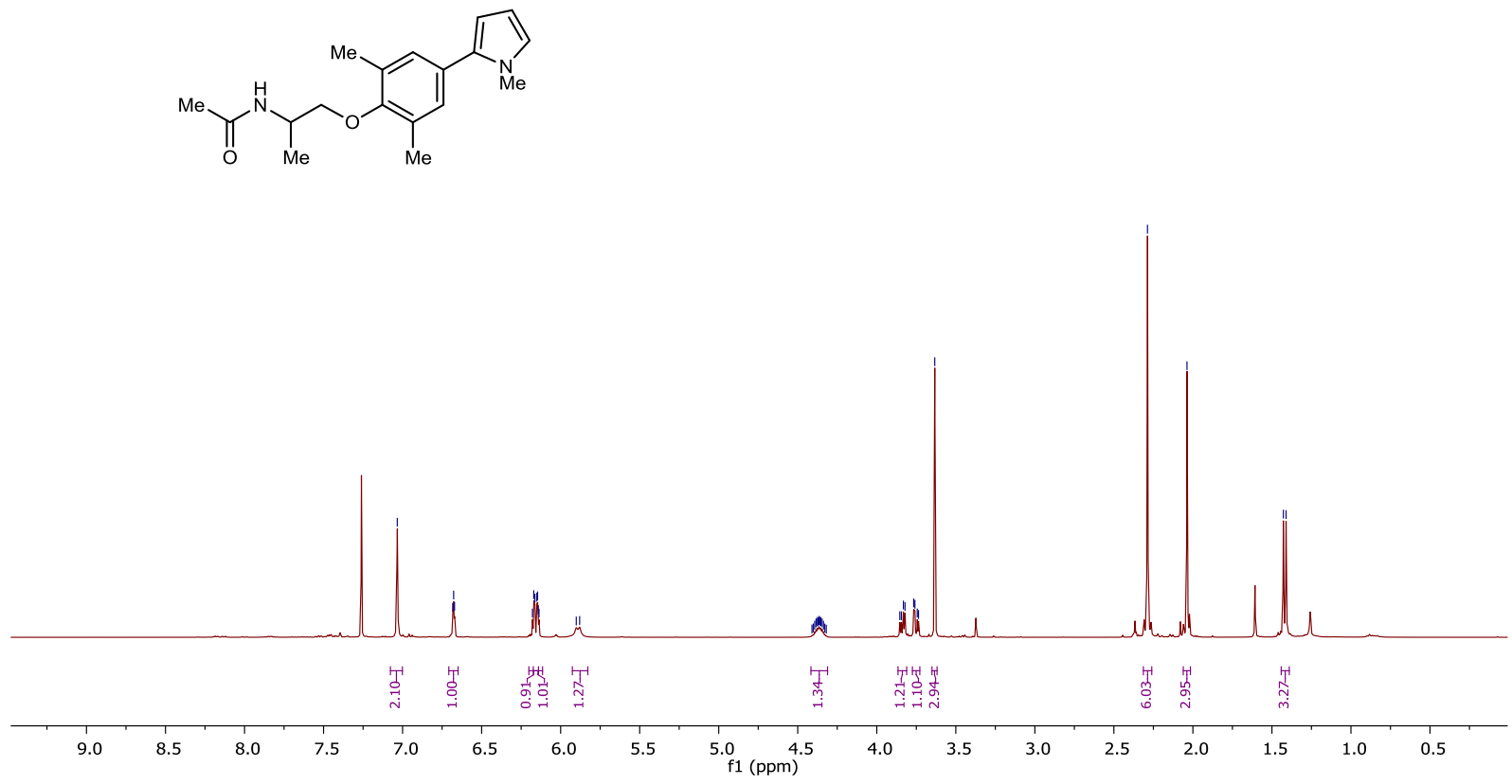

$56-{ }^{13} \mathrm{C}$ NMR $\left(100 \mathrm{MHz}, \mathrm{CDCl}_{3}\right)$

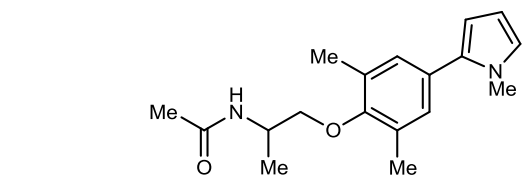

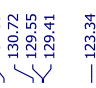

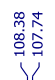

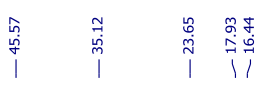

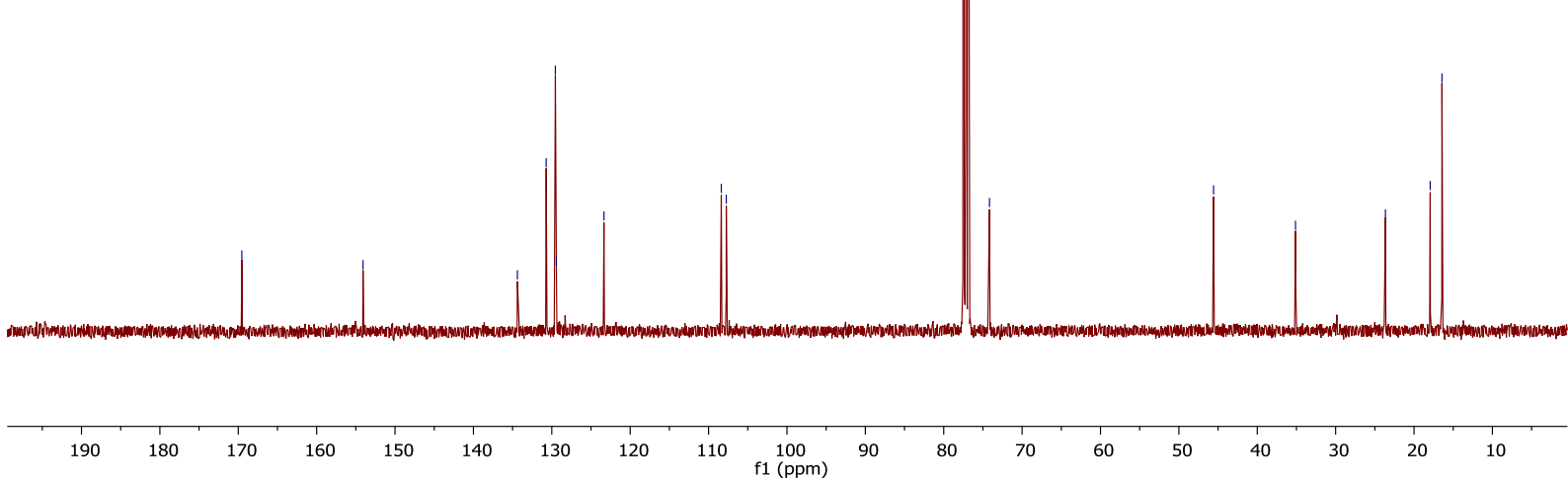

125 
$4-{ }^{1} \mathrm{H}$ NMR $\left(500 \mathrm{MHz}, \mathrm{CDCl}_{3}\right)$

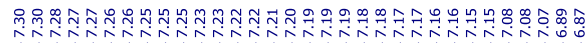<smiles></smiles>

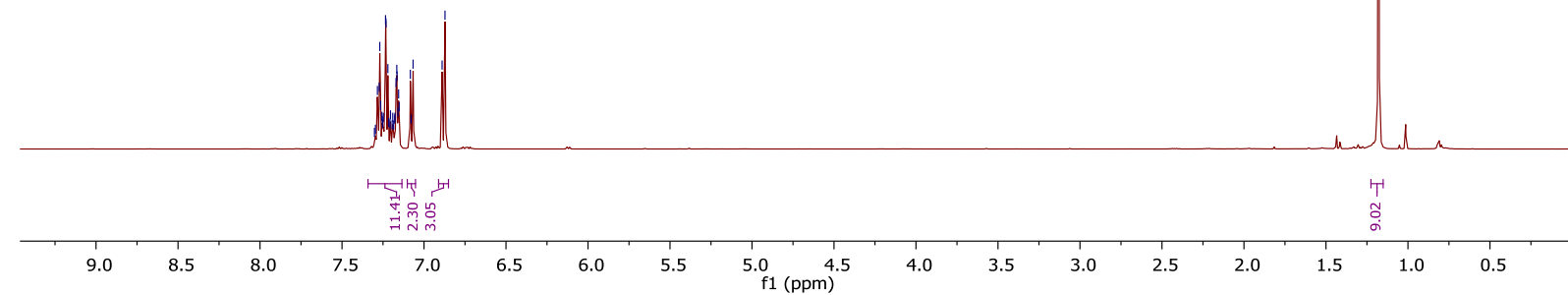

$4-{ }^{13} \mathrm{CNMR}\left(125 \mathrm{MHz}, \mathrm{CDCl}_{3}\right)$

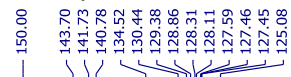<smiles></smiles> 

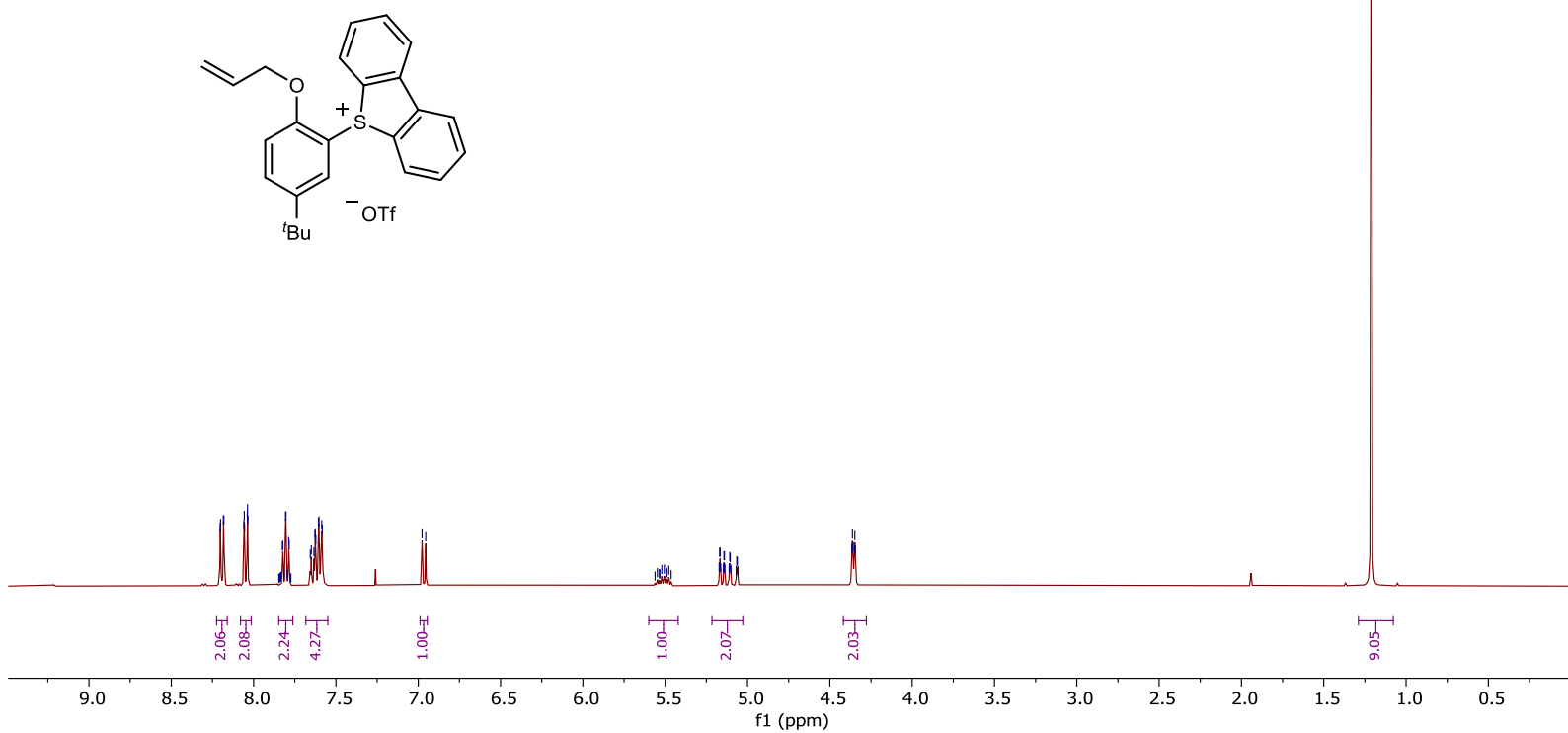

$5-{ }^{13} \mathrm{CNMR}\left(100 \mathrm{MHz}, \mathrm{CDCl}_{3}\right)$

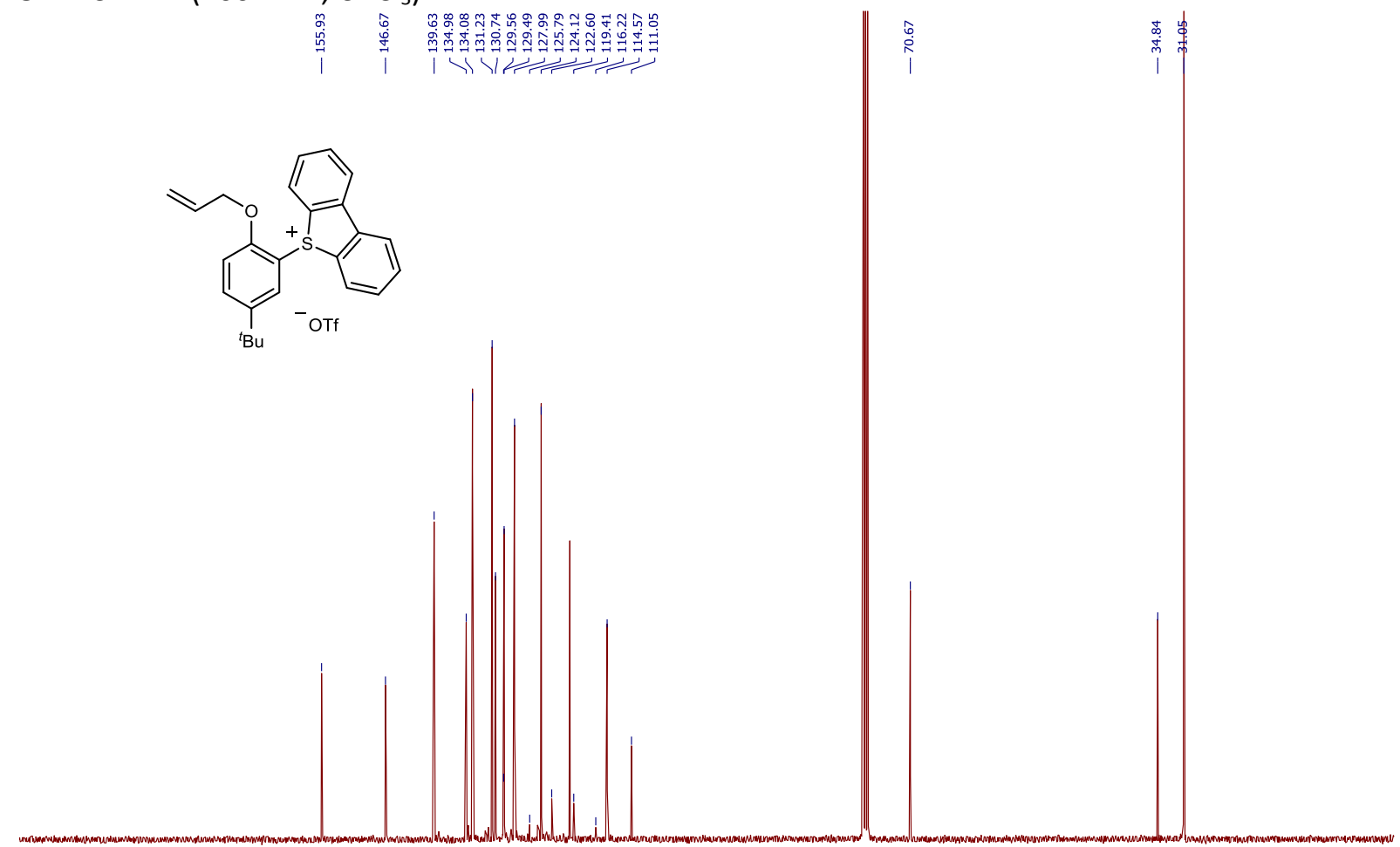

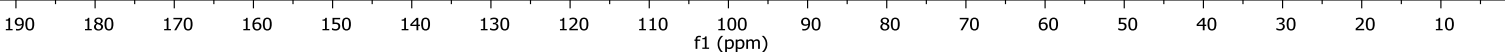


$5-{ }^{19} \mathrm{~F} \mathrm{NMR}\left(376 \mathrm{MHz}, \mathrm{CDCl}_{3}\right)$

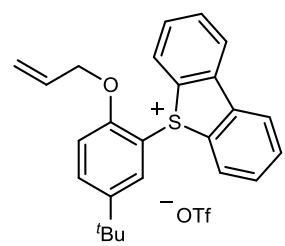

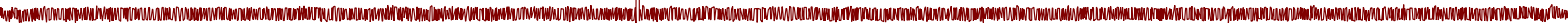

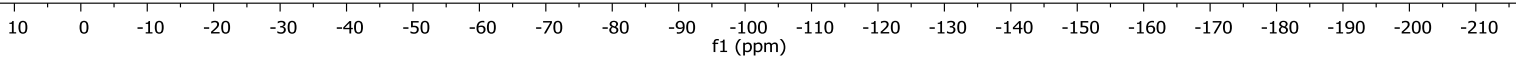


$6-{ }^{1} \mathrm{H}$ NMR $\left(500 \mathrm{MHz}, \mathrm{CDCl}_{3}\right)$

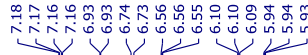

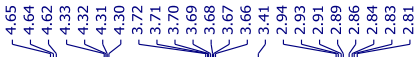

(1)

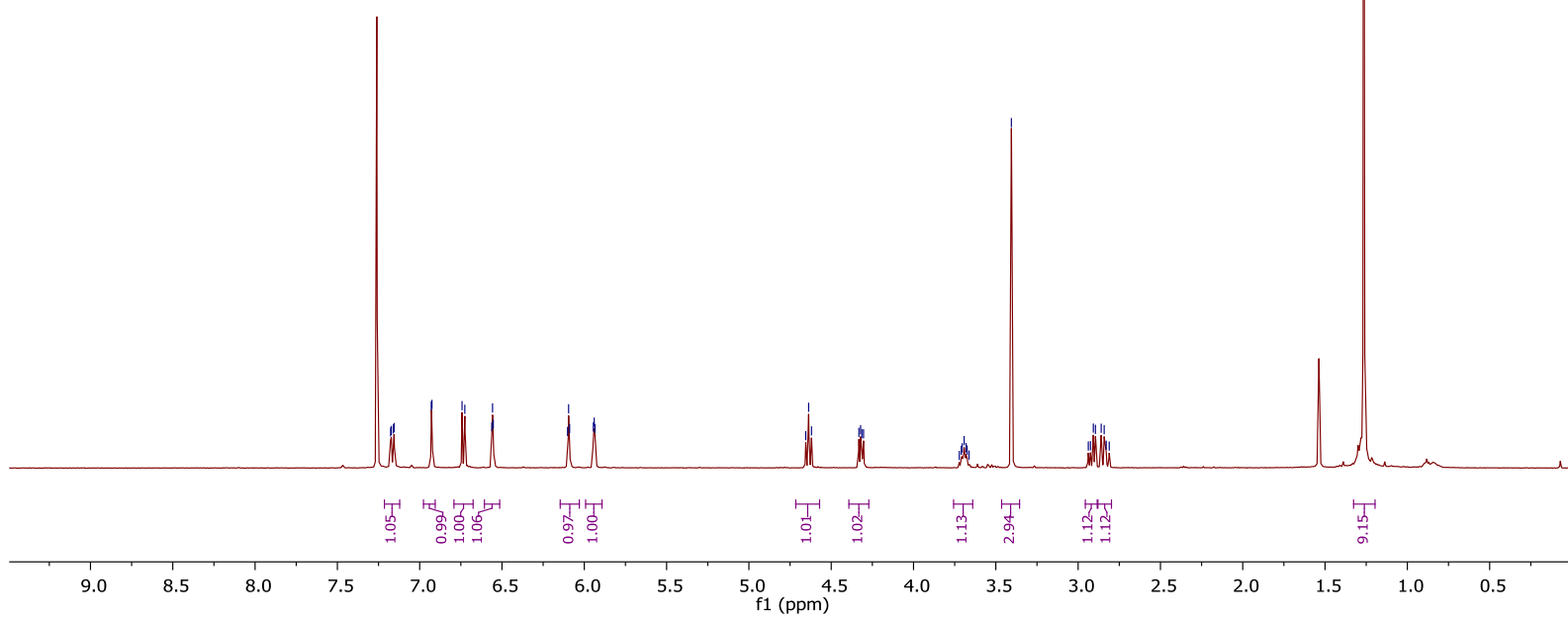

$6-{ }^{13} \mathrm{C}$ NMR (125 MHz, $\mathrm{CDCl}_{3}$ )

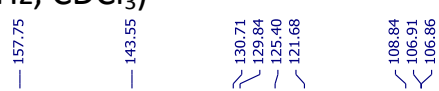

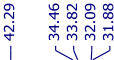

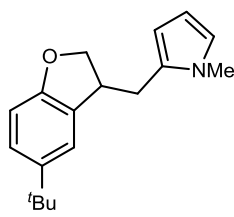

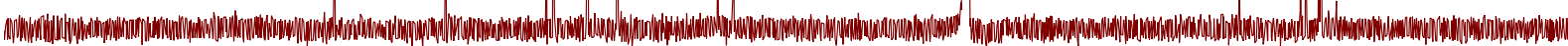

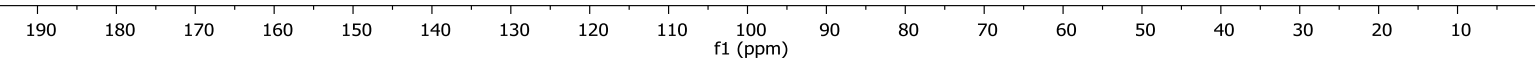




\section{X-RAY CRYSTAL STRUCTURES}

\section{Data Collection}

X-ray data was collected at a temperature of $291 \mathrm{~K}$ on an Agilent Technologies Supernova diffractometer equipped with an Eos CCD detector with MoKa radiation, ( $\lambda=0.71073 \AA$ ) for compound 2.

X-ray data was collected at a temperature of $100 \mathrm{~K}$ on a Rigaku FR-X diffractometer with an Atlas HP6000 detector with MoKa radiation, $(\lambda=0.71073 \AA)$ for compound 53.

Both diffractometers were equipped with an Oxford Cryosystems Cobra nitrogen flow gas system. Data was measured using CrysAlisPro suite of programs.

\section{Crystal structure determinations and refinements}

X-ray data were processed and reduced using CrysAlisPro suite of programs. The crystal structures were solved and refined against all $F^{2}$ values using the SHELX and Olex 2 suite of programs. ${ }^{17,18}$ All the non-hydrogen atoms were refined anisotropically. Hydrogen atoms were placed in calculated positions refined using idealized geometries (riding model) and assigned fixed isotropic displacement parameters. Some carbon atoms were found disordered and modelled over two positions were possible. In such cases, C-C bond distances were restrained using DFIX and SADI commands. The atomic displacement parameters (adp) of the disordered atoms have been restrained using the RIGU command.

These data sets can be obtained free of charge via www.ccdc.cam.ac.uk/conts/retrieving.html (or from the Cambridge Crystallographic Data Centre, 12 Union Road, Cambridge CB21EZ, UK; fax: (+44)1223 336033; or deposit@ccdc.cam.ac.uk). 
X-ray structure of 5-(4-(tert-Butyl)phenyl)-5H-dibenzo[b, $d]$ thiophen-5-ium

trifluoromethanesulfonate $\mathbf{2}$ - CCDC: 1922367
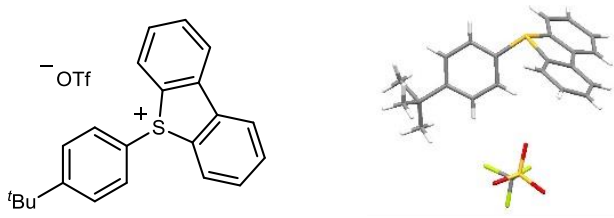

Table A1 Crystal data and structure refinement for $\mathbf{2}$

Identification code

Empirical formula

Formula weight

Temperature/K

Crystal system

Space group

$\mathrm{a} / \AA$

$\mathrm{b} / \AA$

$c / \AA$

$\alpha /^{\circ}$

$\beta /^{\circ}$

$\gamma /^{\circ}$

Volume $/ \AA^{3}$

Z

$\rho_{\text {calc }} \mathrm{g} / \mathrm{cm}^{3}$

$\mu / \mathrm{mm}^{-1}$

$\mathrm{F}(000)$

Crystal size $/ \mathrm{mm}^{3}$

Radiation

$2 \Theta$ range for data collection/ ${ }^{\circ}$

Index ranges

Reflections collected

Independent reflections

Data/restraints/parameters

Goodness-of-fit on $\mathrm{F}^{2}$

Final $R$ indexes $[1>=2 \sigma(I)]$

Final $R$ indexes [all data]

Largest diff. peak/hole / e $\AA^{-3}$
5-(4-(tert-Butyl)phenyl)-5H-dibenzo[ $b, d]$ thiophen-5ium trifluoromethanesulfonate

$\mathrm{C}_{23} \mathrm{H}_{21} \mathrm{~F}_{3} \mathrm{O}_{3} \mathrm{~S}_{2}$

466.52

291

monoclinic

$\mathrm{P} 2{ }_{1} / \mathrm{n}$

13.9567(6)

$8.9349(3)$

19.5441(7)

90

109.050(4)

90

2303.71(16)

4

1.345

0.277

968.0

$0.14 \times 0.14 \times 0.1$

$\operatorname{MoK} \alpha(\lambda=0.71073)$

5.506 to 50.692

$-16 \leq h \leq 16,-10 \leq k \leq 10,-23 \leq \mathrm{I} \leq 20$

26063

$4206\left[R_{\text {int }}=0.0308, R_{\text {sigma }}=0.0236\right]$

$4206 / 99 / 340$

1.059

$\mathrm{R}_{1}=0.0473, \mathrm{wR}_{2}=0.1159$

$\mathrm{R}_{1}=0.0614, \mathrm{wR}_{2}=0.1254$

$0.44 /-0.36$ 
<smiles>Cn1cccc1-c1ccc(NC(=O)c2cccnc2Cl)c(-c2ccc(Cl)cc2)c1</smiles>

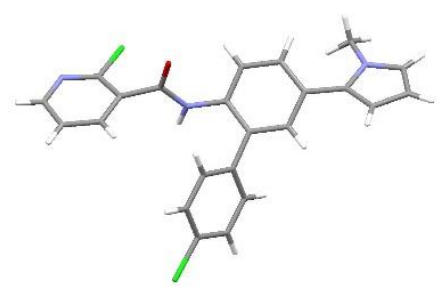

Table A2 Crystal data and structure refinement for $\mathbf{5 3}$

Identification code

Empirical formula

Formula weight

Temperature/K

Crystal system

Space group

$\mathrm{a} / \AA$

$\mathrm{b} / \AA ̊ \AA$

$c / \AA$

$\alpha /^{\circ}$

$\beta /^{\circ}$

$\gamma /{ }^{\circ}$

Volume $/ \AA^{3}$

Z

$\rho_{\text {calc }} \mathrm{g} / \mathrm{cm}^{3}$

$\mu / \mathrm{mm}^{-1}$

$\mathrm{F}(000)$

Crystal size $/ \mathrm{mm}^{3}$

Radiation

$2 \Theta$ range for data collection $/{ }^{\circ}$

Index ranges

Reflections collected

Independent reflections

Data/restraints/parameters

Goodness-of-fit on $\mathrm{F}^{2}$

Final $R$ indexes $[I>=2 \sigma(I)]$

Final $R$ indexes [all data]

Largest diff. peak/hole / e $\AA^{-3}$

Flack parameter
2-Chloro- $N$-(4'-chloro-5-(1-methyl-1H-pyrrol-2-yl)-

[1,1'-biphenyl]-2-yl) nicotinamide

$\mathrm{C}_{23} \mathrm{H}_{17} \mathrm{Cl}_{2} \mathrm{~N}_{3} \mathrm{O}$

422.29

100.0(3)

orthorhombic

Pna2 1

$7.6043(2)$

13.7973(3)

18.5805(4)

90

90

90

1949.44(8)

4

1.439

3.156

872.0

$0.066 \times 0.018 \times 0.015$

CuKa $(\lambda=1.54184)$

7.982 to 136.486

$-8 \leq h \leq 9,-16 \leq k \leq 16,-20 \leq \mathrm{I} \leq 22$

8626

$3358\left[R_{\text {int }}=0.0439, R_{\text {sigma }}=0.0604\right]$

$3358 / 1 / 263$

1.080

$\mathrm{R}_{1}=0.0426, \mathrm{wR}_{2}=0.1112$

$\mathrm{R}_{1}=0.0456, \mathrm{wR}_{2}=0.1129$

$0.21 /-0.44$

$-0.001(15)$ 


\section{REFERENCES}

[1] Aranzaes, J. R., Daniel, M-C. \& Astruc, D. Metallocenes as references for the determination of redox potentials by cyclic voltammetry - Permethylated iron and cobalt sandwich complexes, inhibition by polyamine dendrimers, and the role of hydroxy-containing ferrocenes. Can. J. Chem. 84, 288-299 (2006).

[2] Discekici, E. H., Treat, N. J., Poelma, S. O., Mattson, K. M., Hudson, Z. M., Luo, Y., Hawker, C. J. \& De Alaniz, J. R. A highly reducing metal-free photoredox catalyst: design and application in radical dehalogenations. Chem. Commun. 51, 11705-11708 (2015).

[3] Wang, H. \& Jui, N. T. Catalytic Defluoroalkylation of Trifluoromethylaromatics with Unactivated Alkenes. J. Am. Chem. Soc. 140, 163-166 (2018).

[4] Cismesia, M. A. \& Yoon, T. P. Characterizing chain processes in visible light photoredox catalysis. Chem. Sci. 6, 5426-5434 (2015).

[5] He, Z., Pulis, A. P. \& Procter, D. J. The Interrupted Pummerer Reaction in a SulfoxideCatalyzed Oxidative Coupling of 2-Naphthols. Angew. Chem. Int. Ed. 58, 7813-7817 (2019).

[6] Jessen, H. J., Schulz, T., Balzarini, J. \& Meier, C. Bioreversible Protection of Nucleoside Diphosphates. Angew. Chem. Int. Ed. 47, 8719-8722 (2008).

[7] Li, G., Lei, P. \& Szostak, M. Transition-Metal-Free Esterification of Amides via Selective N-C Cleavage under Mild Conditions. Org. Lett. 20, 5622-5625 (2018).

[8] Rogers, G. A. \& Bruice, T. C. Synthesis and evaluation of a model for the so-called chargerelay system of the serine esterases. J. Am. Chem. Soc. 96, 2473-2481 (1974).

[9] Dittmann, M., Graupner, F. F., Maerz, B., Oesterling, S., Devivie-Riedle, R., Zinth, W., Engelhard, M. \& Luettke, W. Photostability of 4,4'-Dihydroxythioindigo, a Mimetic of Indigo. Angew. Chem. Int. Ed. 53, 591-594 (2014).

[10] Brenna, E., Fronza, G., Fuganti, C., Gatti, F. G., Pinciroli, M. \& Serra, S. Differentiation of Extractive and Synthetic Salicin. The ${ }^{2} \mathrm{H}$ Aromatic Pattern of Natural 2-Hydroxybenzyl Alcohol. J. Agric. Food Chem. 52, 7747-7751 (2004).

[11] Berger, J., Flippin, L. A., Hunter, J. C., Loughhead, D. G. \& Weikert, R. J. Syntex Inc. Treatment of neuropathic pain. US5688830. (1997)

[12] Yue, H., Guo, L., Lee, S-C., Liu, X. \& Rueping, M. Selective Reductive Removal of Ester and Amide Groups from Arenes and Heteroarenes through Nickel-Catalyzed C-O and C-N Bond Activation. Angew. Chem. Int. Ed. 56, 3972-3976 (2017).

[13] Meyer, A. U., Slanina, T., Heckel, A. \& König, B. Lanthanide lons Coupled with Photoinduced Electron Transfer Generate Strong Reduction Potentials from Visible Light. Chem. Eur. J. 23, 7900-7904 (2017).

[14] Tobisu, M., Furukawa, T. \& Chatani, N. Visible Light-mediated Direct Arylation of Arenes and Heteroarenes Using Diaryliodonium Salts in the Presence and Absence of a Photocatalyst. Chem. Lett. 42, 1203-1205 (2013). 
[15] Martin, R., Risacher, C., Barthel, A., Jäger, A., Schmidt, A. W., Richter, S., Böhl, M., Preller, M., Chinthalapudi, K., Manstein, D. J., Gutzeit, H. O. \& Knölker, H-J. Silver(I)-Catalyzed Route to Pyrroles: Synthesis of Halogenated Pseudilins as Allosteric Inhibitors for Myosin ATPase and X-ray Crystal Structures of the Protein-Inhibitor Complexes. Eur. J. Org. Chem. 4487-4505 (2014).

[16] Donck, S., Baroudi, A., Fensterbank, L., Goddard, J-P. \& Ollivier, C. Visible-Light Photocatalytic Reduction of Sulfonium Salts as a Source of Aryl Radicals. Adv. Synth. Catal. 355, 1477-1482 (2013).

[17] Sheldrick, G. M. SHELXT - Integrated space-group and crystal-structure determination. Acta Crystallogr. Sect. A Found. Crystallogr. 71, 3-8 (2015).

[18] Dolomanov, O. V., Bourhis, L. J., Gildea, R. J., Howard, J. A. K. \& Puschmann, H. OLEX2: a complete structure solution, refinement and analysis program. J. Appl. Crystallogr. 42, 339341 (2009). 
